1996

\title{
The technical and economic feasibility of mining the near-earth asteroids
}

Mark Joseph Sonter

University of Wollongong

\section{Recommended Citation}

Sonter, Mark Joseph, The technical and economic feasibility of mining the near-earth asteroids, Master of Science (Hons.) thesis, Department of Physics, University of Wollongong, 1996. http://ro.uow.edu.au/theses/2862 


\section{NOTE}

This online version of the thesis may have different page formatting and pagination from the paper copy held in the University of Wollongong Library.

\section{UNIVERSITY OF WOLLONGONG}

\section{COPYRIGHT WARNING}

You may print or download ONE copy of this document for the purpose of your own research or study. The University does not authorise you to copy, communicate or otherwise make available electronically to any other person any copyright material contained on this site. You are reminded of the following:

Copyright owners are entitled to take legal action against persons who infringe their copyright. A reproduction of material that is protected by copyright may be a copyright infringement. A court may impose penalties and award damages in relation to offences and infringements relating to copyright material. Higher penalties may apply, and higher damages may be awarded, for offences and infringements involving the conversion of material into digital or electronic form. 


\title{
The Technical and Economic Feasibility of Mining the Near-Earth Asteroids
}

\author{
A thesis submitted in fulfilment of the requirements for \\ the award of the degree of \\ Master of Science (Honours) \\ from \\ University of Wollongong
}

by

Mark J Sonter, B.Sc., M.App.Sc.

Department of Physics and Department of Civil and Mining Engineering 1996 


\section{Certification:}

This is to certify:

(i) that this thesis is solely the work of the candidate;

(ii) that this thesis has not been submitted for a degree to any other university or institution.

M J Sonter 


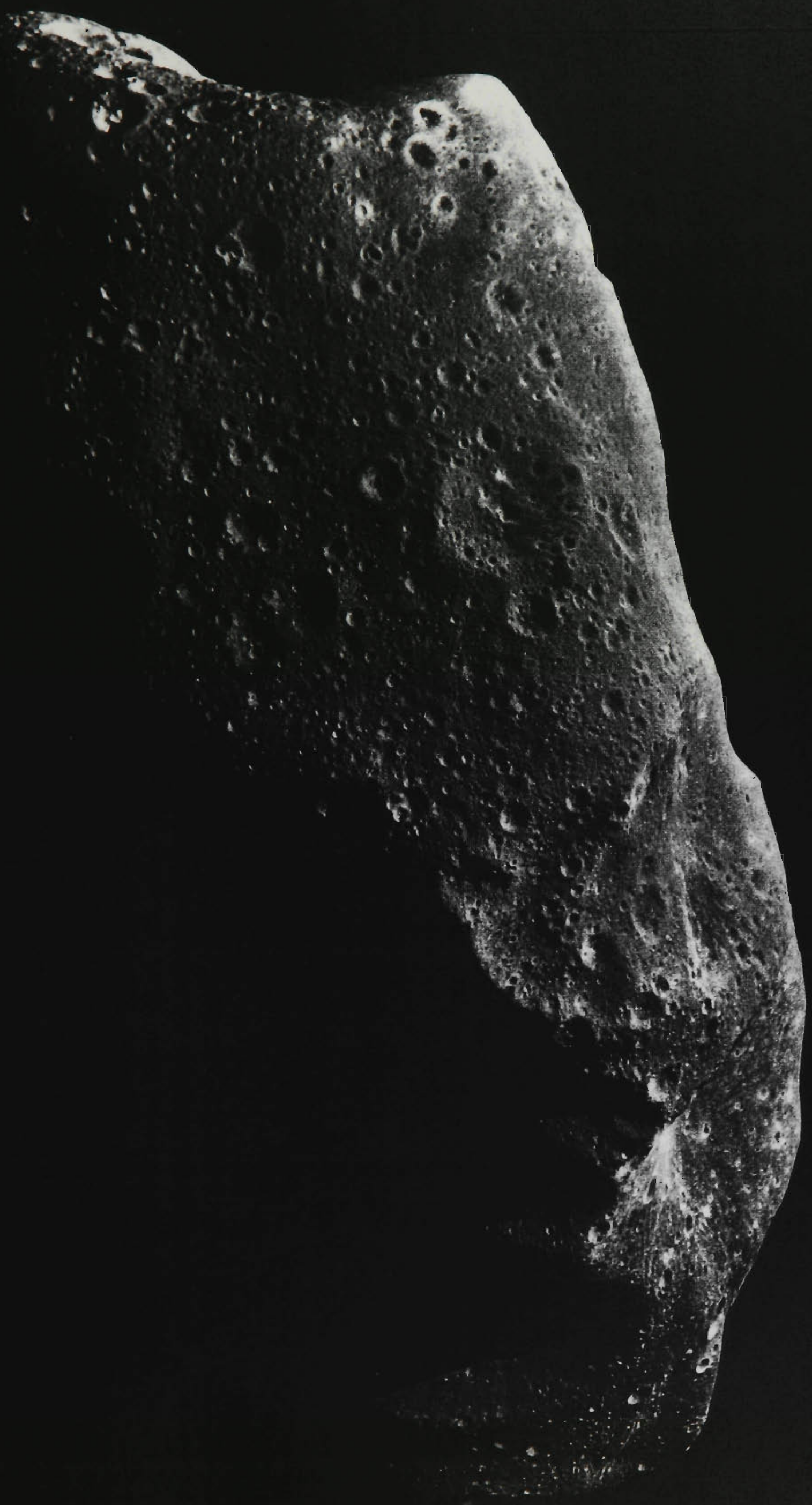




\section{Acknowledgments:}

I wish to acknowledge the interest and support of my supervisors, Dr Bill Zealey, Head of the Department of Physics, and Prof Raghu Singh, Head of the Department of Civil and Mining Engineering, University of Wollongong.

I also wish to acknowledge the assistance of Dr John Lewis, Co-Director, Space Engineering Research Center for the Utilization of Local Planetary Resources, Lunar and Planetary Laboratory, University of Arizona, who provided my initial contacts with colleagues in the US.

Most importantly, I wish to thank my wife Wendy for her faith, support and many hours of help; and my daughters Katrina and Samantha for their help and affection.

Mark Sonter

\section{Copyright:}

The author retains full copyright privileges over this work

M J Sonter

Frontispiece: Asteroid 243 Ida, a Main Belt S-type asteroid, photographed at a distance of $2400 \mathrm{~km}$ by the Galileo Jupiter probe on 28th August 1993. 


\section{Abstract}

Future space industrialization will prompt the search for in-space resources, for construction and propellant mass.

This thesis reviews the literature regarding space resources, and notes the tremendous expansion in knowledge of the Near-Earth asteroids over the last decade, in regard to their population, compositions, and accessibility, making them primary targets of interest.

The literature highlights, and this thesis addresses, the need to consider details of potential mining and processing methods.

The literature also highlights the need for rigorous ways of comparing alternative hypothetical projects and deciding between competing targets, and competing mining, processing, propulsion, and power system choices, which all interact in complex ways.

This thesis identifies that the most useful high-level design-driver for assistance in making these choices and comparisons is the project Expectation Net Present Value, and produces flow diagrams, equations, and a calculation process enabling easy NPV calculation for various target orbit types, mission types, and system choices.

Examples are worked, using reasonable numbers for equipment mass and throughput, and basic celestial mechanics constraints. The conclusion is reached that robotic resource recovery from NEAs is technically feasible in the near term, and that the returned product can potentially be highly profitable, given an in-space market of some thousands of tonnes per year, in competition against Earth-launch costs of several hundred dollars per kilogram. 


\section{Glossary}

apollo asteroid

amor asteroid

aten asteroid

aphelion

carbonyl

hyperbolic velocity

Hohmann transfer orbit

impulsive

kerogen

perihelion

pyrolysis

regolith

synodic period

transfer orbit

trojan

volatiles asteroid with perihelion $\mathrm{q}<1.017 \mathrm{AU}$ and semi-major axis $\mathrm{a}>1.0 \mathrm{AU}$

asteroid with perihelion $1.017 \mathrm{AU}<\mathrm{q} \leq 1.3 \mathrm{AU}$

asteroid with semi-major axis $\mathrm{a}<1.0 \mathrm{AU}$ and aphelion $\mathrm{Q}$ $>0.983 \mathrm{AU}$

Q; the point on an orbit that is most distant from the Sun a compound of a metal with carbon monoxide

the velocity of an object relative to a planet (in this thesis, the Earth) when it is outside that body's gravity well.

the most energy-efficient transfer trajectory between two coplanar orbits: it is an ellipse, tangent to the two orbits between which the body is transferring.

a change in velocity that is imparted in a short period of time relative to the total trajectory duration

the solid hydrocarbon in oil-shale

$\mathrm{q}$; the point on an orbit which is closest to the Sun

generation of chemical species by thermal decomposition

surface fragmented rocky debris blanketing the Moon and small solar system objects

period of a body with respect to the Earth

trajectory from one body to another

an object which is trapped in a stable orbit 60 degrees ahead of or behind the primary body as it orbits the Sun.

gases that can be released from comet cores by heating: (and hypothetically, from asteroids which are analogues of carbonaceous chondrites) water, carbon dioxide and monoxide, methane, ammonia, and hydrogen cyanide. 


\section{Abbreviations}

$\begin{array}{ll}\text { AN } & \text { Ascending Node } \\ \text { AU } & \text { Astronomical Unit, equals the semimajor axis of Earth's orbit } \\ \text { DN } & \text { Descending Node } \\ \text { FOM } & \text { Figure of Merit } \\ \text { GEO } & \text { Geostationary Earth Orbit } \\ \text { HEEO } & \text { Highly Elliptical Earth Orbit } \\ \text { ISPP } & \text { In-Situ Propellant Production } \\ \text { IRR } & \text { Internal Rate of Return } \\ \text { LEO } & \text { Low Earth Orbit } \\ \text { LOX } & \text { Liquid Oxygen } \\ \text { LH } & \text { Liquid Hydrogen } \\ \text { MPBR } & \text { Mass Payback Ratio } \\ \text { NEA, NEO } & \text { Near-Earth Asteroid, Near-Earth Object } \\ \text { NPV } & \text { Net Present Value } \\ \text { NiFe } & \text { Nickel-Iron } \\ \text { PGM } & \text { Platinum Group Metals } \\ \text { ppm } & \text { parts per million } \\ \text { SSPS } & \text { Satellite Solar Power Station } \\ \text { SSTO } & \text { Single Stage to Orbit } \\ \text { SNC } & \text { Vertical Takeoff and Landing } \\ \text { VTOL } & \end{array}$




\section{Mathematical Symbols}

a

e

i

q

Q

$\Delta \mathrm{v}$

$\mathrm{I}_{\mathbf{s p}}$

$v_{e}$

$\Delta \mathrm{v}_{\mathrm{DS}}$

$\mathrm{C}_{3}$ semi-major axis (of orbit)

eccentricity (of orbit)

inclination to the ecliptic (of orbital plane)

perihelion (of orbit)

aphelion (of orbit)

velocity change

specific impulse of rocket exhaust (seconds)

exhaust velocity (of rocket)

deep-space rendezvous velocity

hyperbolic departure velocity squared $\left(\mathrm{km}^{2} / \mathrm{s}^{2}\right)$ 
List of Tables

Page

$1.1 \quad$ Mission Velocity Requirements ............................................ 7

1.2 Spectral Type, Inferred Mineralogy, \& Potential Products ..................... 11

1.3 Possible Products and Sources.................................................. 13

$1.4 \quad$ Propulsion and Power Choices ............................................. 14

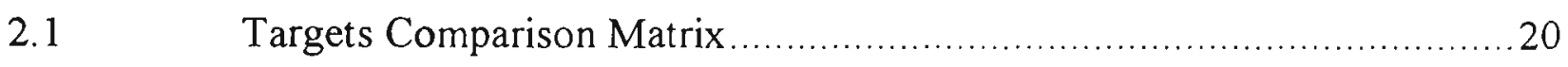

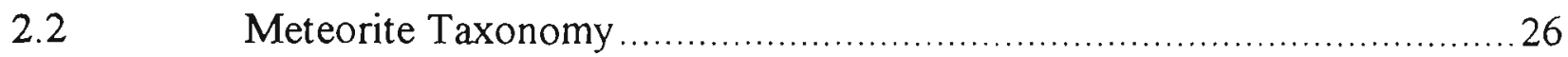

Asteroid Observation Methods ............................................... 30

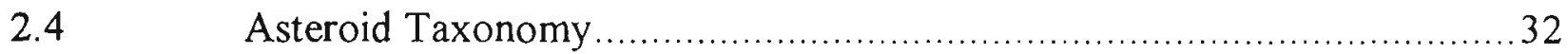

2.5 Suspected Comet-Origin Asteroids ............................................. 35

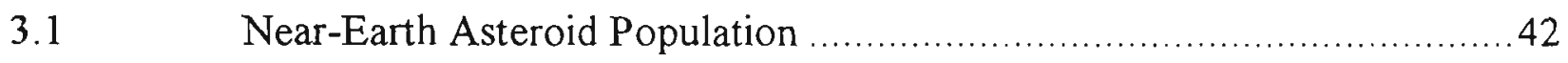

Accessibility of Near-Earth Asteroids ......................................... 44

3.3 Minimum Eccentricity Near-Earth Asteroids .................................46

Kuck's List of Short-Period Comet Targets .................................... 48

6.1 Matrix of Product-Process Options ........................................... 81

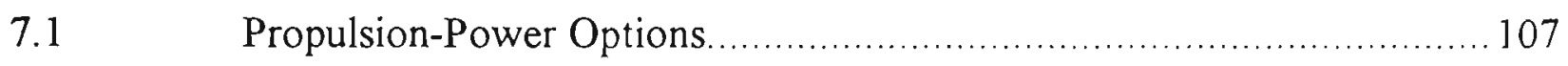

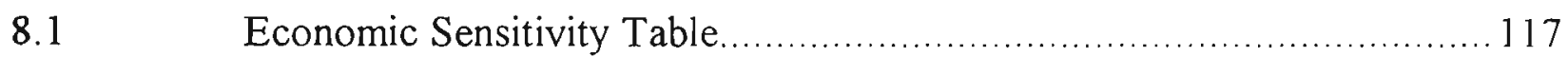

8.2 Present Value versus Time to Payment ....................................... 118

9.1 Nereus Orbital Parameters ................................................... 132

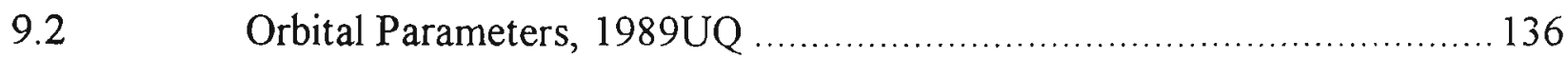

9.3 Aten Missions Comparison ............................................. 141

9.4 Orbital Parameters, 1991VG ................................................ 142

9.5 Orbital Parameters, Wilson-Harrington .................................. 145

9.6 Orbital Parameters, p/DuToit-Hartley ....................................... 149 


\section{List of Figures}

Present Launch Costs .5 


\section{CONTENTS}

Acknowledgments .i

Abstract ii

Certification iii

Glossary iv

Abbreviations .. V

Mathematical Symbols vi

List of Tables vii

List of Figures viii

\section{Chapter 1 Introduction}

1.1 Background to the Concepts of Space Mining .................................. 1

1.1.1 Desirability of Space Development....................................1

1.1.2 Commercial 'Drivers' for Space Industrialization .....................2

1.1.3 Potential Future Market for Mass-in-Orbit ..............................4

1.1.4 Accessibility of Non-Terrestrial Resources............................6

1.1.5 In-Situ Propellant Production.............................................. 8

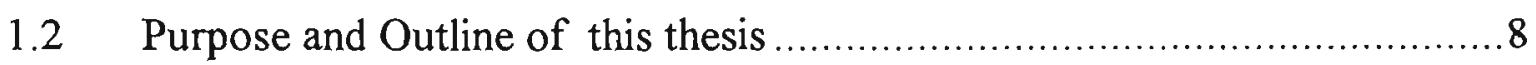

1.2.1 Review of Asteroid Geology \& Resources. ........................ 10

1.2.2 Asteroid Accessibility and Mission Profiles. ............................ 11

1.2.3 Concepts for Mining, Processing, Power, \& Propulsion. ........... 12

1.2.4 Asteroid Mining Project Feasibility \& Selection Criteria ............ 14

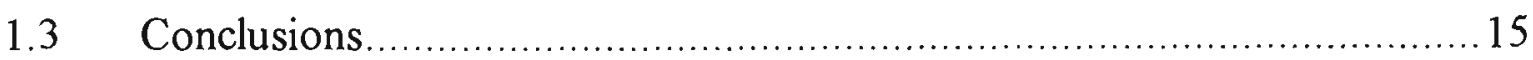

\section{Chapter 2. Asteroid Resources}

2.1 Attractiveness of Asteroids c.f. the Moon and Phobos / Deimos ............. 16

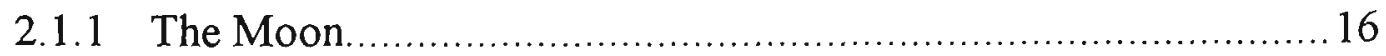

2.1.2 The moons of Mars.................................................. 18

2.1.3 The Near-Earth Asteroids .......................................... 19

2.1.4 NEAs Attractiveness................................................ 21 
2.2 Asteroid and Comet Geology and Resources .................................. 22

2.2.1 Meteorite Mineralogy \& Taxonomy....................................25

2.2.2 Asteroid taxonomy and inferred compositions ........................30

2.2.3 Comet origin of some asteroids ..................................... 35

2.2.4 Comet models and theorised mantle structure ....................... 36

2.3 Conclusion: Appears to be a continuum from "cryptocometary" through carbonaceous to silicate and metallic asteroidal bodies.

\section{Chapter 3: Asteroid "Geography"}

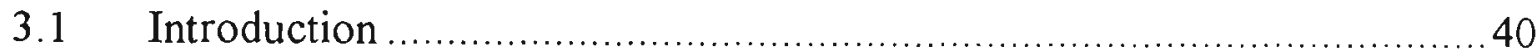

3.2 The Earth-Approaching or Near-Earth Asteroids ............................... 40

3.3 Databases of known NEAs................................................... 41

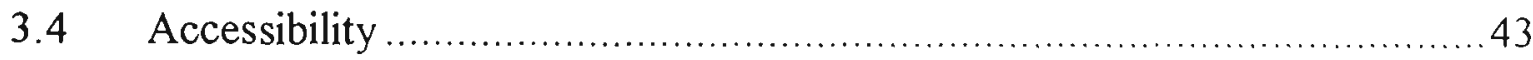

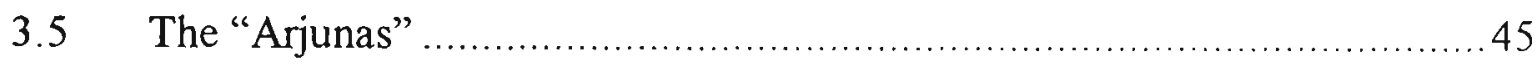

3.6 Short-Period Comet targets ................................................... 48

3.7 Conclusion: Large \& growing no. of NEAs; many possible "orebodies"; many NEAs more accessible than the Moon.

\section{Chapter 4: Orbital Mechanics}

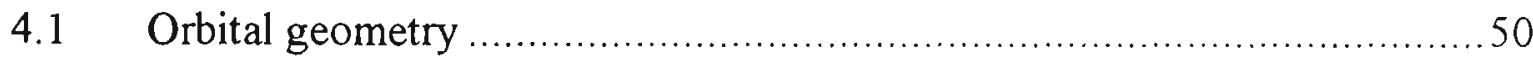

4.2 Mission velocity as measure of "accessibility" \& propulsion demand....... 50

4.3 Delta-v Calculations ........................................................... 52

4.3.1 Shoemaker and Helins' Figure of Merit formulae ...................52

4.3.2 Empirical "polynomial fit" formulae ................................. 55

4.4 Hohmann 'first principle' calculations; energy cost of plane change .........56

\section{Chapter 5: Mission Plans and Trajectories}

5.1 Hohmann missions and timing considerations...........................60

5.2 Taxonomy of alternative Project Mission Types...............................61 61

5.2 .1 “Apollo-Type missions .................................................61

5.2.2 Short Period Comet missions............................................64 64

5.2 .3 “Aten-Type" missions...............................................65 
5.2.4 "Arjuna-Type" missions. 66

5.2.5 High inclination, Low eccentricity missions ...........................68

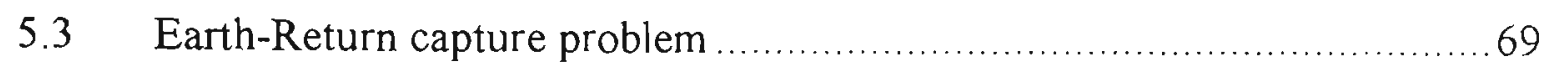

5.4 Arguments against multiple return missions ................................. 70

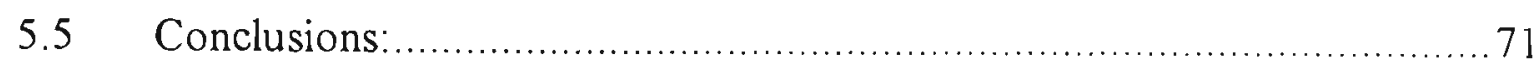

(i) Phasing requirements inhibit "multiple return" missions. ...........71

(ii) Excessive $v_{\text {hyp }}$ on Earth-Return is as detrimental to project as high $\Delta v_{D S}$, or $\Delta v$ for return departure.

(iii) Non-Hohmann transfer best for low-e; Hohmann transfer with aphelion mining season best for high-e targets

\section{Chapter 6: Engineering Choices : Mining and Processing}

6.1 Mining in Zero-Gravity: anchoring and reaction force ........................ 72

6.1.1 Mining in Zero Gravity ............................................... 72

6.1.2 Possible Collection \& Extraction Methods .............................. 73

6.1.2.1 Surface Mining .......................................... 74

6.1.2.2 Underground Extraction ............................... 75

6.1.2.3 In-situ Extraction ........................................... 76

6.2 Metallurgical Processing / Beneficiation........................................ 78

6.2.1 Comminution.......................................................... 78

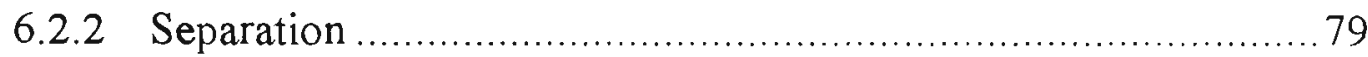

6.2.3 Processing - Influence of Product Type ............................... 79

6.2.4 Processing - Influence of Feedstock ................................. 80

6.3 Feasible Products \& Processing Methods........................................... 82

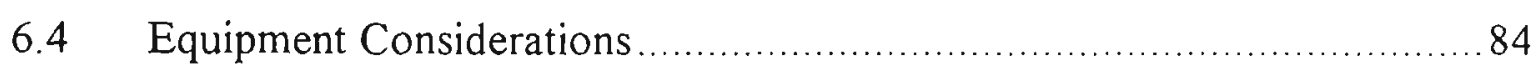

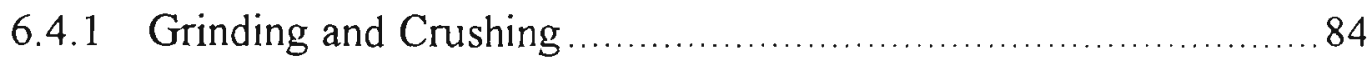

6.4.2 Materials Handling...................................................... 85

6.4.3 Heat Processing ........................................................ 86

6.4.3.1 Melting Ice ........................................... 86

6.4.3.2 Devolatilizing Carbonaceous Chondrite Material ......87

6.4.3.3 Further Heating Considerations ............................ 89

6.4.4 Masses and Throughputs ............................................ 91

6.5 Conceptual Process Flowsheet .............................................. 92 
6.6 Kuck process for extraction of comet core volatiles ............................95

6.7 System integration and mass budget .............................................. 99

6.8 Control: telepresence/remote control and machine autonomy ............... 100

6.9 Conclusions: Product-Process Matrix has been developed to guide project choices; Mass estimates for process equipment

\section{Chapter 7: Engineering Choices : Propulsion and Power}

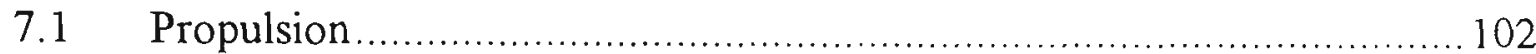

7.1.1 Impulsive (High Thrust) Systems .................................. 103

7.1.2 Low Thrust Systems ................................................... 105

7.2 Power: Solar thermal, solar photovoltaic; or nuclear........................... 106

7.3 Conclusions: Power-Propulsion "Matrix" for sensible system

choices; solar-thermal power and propulsion for initial missions 108

\section{Chapter 8: Project Selection Criteria.}

8.1 Economic Analyses.

8.1.1 Findings from the Literature .......................................... 109

8.1.2 Simple Economic Feasibility Example .............................. 112

8.2 Technical and Economic linkages: the "Spider Diagram" ................... 113

8.3 Net Present Value Discussion and Formula Derivation........................ 115

8.3.1 Breakeven Analysis Comments ......................................... 116

8.3.2 Sensitivity Analysis .................................................. 117

8.3.3 Reliability / Probability Analysis ................................... 118

8.3.4 NPV Derivation and Calculation Process .......................... 118

8.4 Conclusions: NPV should be used to guide project conceptual design, as in terrestrial mining projects.

\section{Chapter 9. Project Examples and Calculations.}

9.1 "Apollo-type" mission to 1989ML......................................... 123

9.2 "Apollo-type" mission to 1982DB Nereus ................................... 132

9.3 “Aten-type" mission to 1989UQ ............................................... 136

9.4 Mission to an "Arjuna", 1991VG ............................................ 142 
9.5 Wilson-Harrington using Kuck process................................... 145

9.6 Comet perihelion mission to $\mathrm{p} / \mathrm{du}$ Toit-Hartley ................................. 149

9.7 Comet perihelion mission to $\mathrm{p} / \mathrm{How}$ ell .................................. 151

9.8 Hypothetical "Arjuna" mission ................................................ 153

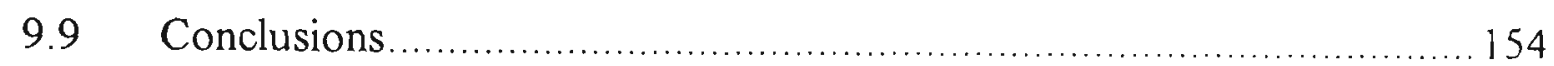

Chapter 10: General Conclusions and Further Work

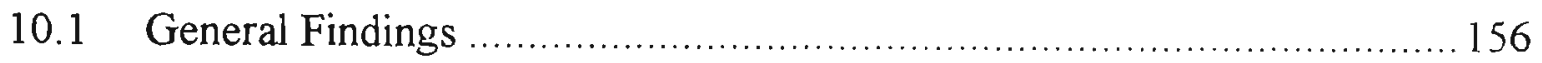

10.2 Information and Technology Gaps Identified for Further Work ............ 159

\section{Appendices:}

1. Legal Regime for Asteroid Mining Tenure ......................................... 160

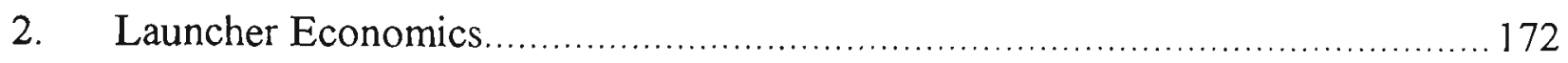

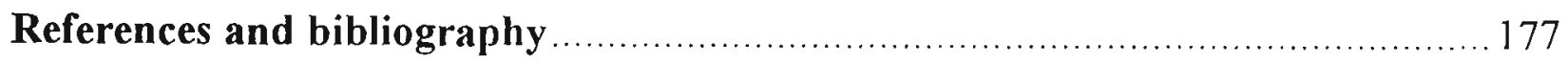

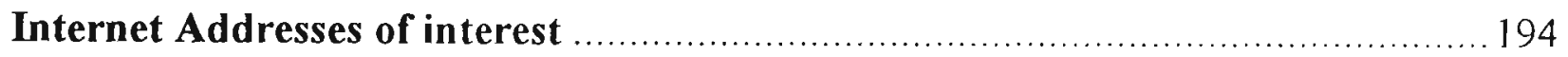

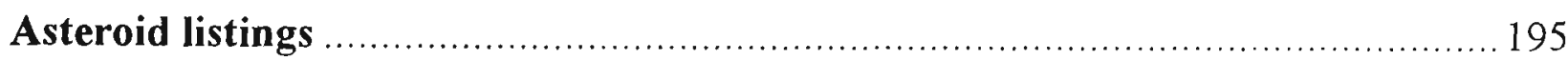




\section{The Technical and Economic Feasibility of Mining the Near-Earth Asteroids.}

M J Sonter, Dept of Physics and Dept of Civil \& Mining Engineering, University of Wollongong, New South Wales, Australia.

\section{Chapter 1: Introduction}

This thesis reviews the literature and concepts relating to mining the Near-Earth Asteroids for supply of resources to future in-space industrial activities. It develops a standard approach for carrying out Preliminary Feasibility Studies on proposals for asteroid resource recovery projects.

\subsection{Background to the Concept of Space Mining.}

\subsubsection{The Desirability of Space Development}

Any considerations of the long-term prosperity and indeed survival of humanity must take into account possible cosmic catastrophes, such as impact by asteroid or comet (Gehrels, 1994; and Chapman \& Morrison, 1994). The need to develop a capability to deflect such potential impactors strongly suggests that mankind needs to be a spacecapable, or in fact a space-colonizing species, rather than a species restricted in its ecological range only to the Earth.

Similarly, considerations of access to the almost unlimited energy and material resources of the solar system also point towards space colonization, focussing initially on the plentiful and diverse material resources in the asteroids (Lewis \& Lewis, 1987).

The technology needed to avert comet or asteroid impact is similar to that needed to recover the probable resources contained in these bodies. Thus it is desirable to develop asteroidal resources, both to achieve wanted outcomes (namely space industrialization, species security, and long term prosperity) and to build the capacity to avert disaster. 
The effect on the popular consciousness of realising that the species range is not restricted to the surface of the Earth, but potentially extends throughout the Solar System, will be profound. The "Limits to Growth" paradigm will be replaced by an optimistic view of an increasingly prosperous, capable, and widespread, and therefore more secure humanity colonizing the Moon, the asteroids, Mars, and the moons of Jupiter and Saturn, and utilizing their resources. This clearly needs to be tested against technical and economic principles as to its achieveability.

\subsubsection{Potential Commercial 'Drivers' for Space Industrialization}

The future industrialization, settlement, and utilisation of space is likely to be brought about primarily by increasing commercial activities in space. In the near term (e.g. the next 20 years) the following reasonably predictable space commercial activities could develop.

Commercial space operations presently provide trunkline communications, direct broadcast TV, navigation, remote sensing, and meteorological services worth several billion dollars per year from an in-space satellite assets investment estimated to be about 50 billion dollars. Space based personal communications is imminent with the introduction of low earth orbit (LEO) satellite based mobile phone systems: "worldphones" such as Motorola's Iridium system. Capital expenditure for the Gates / McCaw Teledesic system, with a constellation of some 800 satellites, is in the order of $\$ 10$ billion.

At present, each satellite investment is rendered useless when it runs out of stationkeeping propellant. "The present annual consumption of propellant to boost communications satellites from low earth orbit (LEO) to geostationary orbit (GEO) is about 250 tonnes. At the present cost of lifting propellant to LEO of about $\$ 8000$ per kilogram, this amounts to about $\$ 2000$ million dollars per year." (Kuck, 1995). Thus, inspace refuelling and refurbishment of this enormous investment is a likely near-term development, using some sort of remote controlled in-orbit refueller. 
It has been speculated that in-space industry may include commercial space-based production of high value pharmaceuticals, semiconductors, ultra-pure crystals, and exotic alloys. Although work has been done on the Russian "Mir" space station (in orbit since 1986) and on various Shuttle Flights, commercialization has been severely delayed by problems of restricted and costly access to space via the Space Shuttle, particularly after the Challenger disaster.

A more speculative, but technically sound driver for commercial space based activities, is the concept of large orbiting satellite solar power stations (SSPS), initially proposed by Dr Peter Glaser of Arthur D Little in the late 1970s. (For a good review of early work, see Glaser, 1982).. Such satellites would collect solar power, convert it to microwaves, and beam it to receiving antennae on Earth where the energy would be rectified, converted to AC power, and fed into the electricity grid.

This concept is again receiving active consideration, after a decade of disinterest: the Japanese are carrying out formal studies into an equatorial orbit SSPS pilot plant, titled SPS2000, to orbit at $1100 \mathrm{~km}$ altitude (Nagatomo, 1996). A full-scale Geosynchronous orbit Solar Power Station (of say 5GW) would mass many thousands of tonnes. An SPS constructed using non-terrestrial materials, sourced from the Moon or the asteroids, could save up to $99 \%$ of the earth-launched mass, and hence earth-launch cost, with concomitant total increased mass of less than $8 \%$ (Space Studies Institute, 1986).

Another speculative, but again technically sound proposal for large scale activity in orbit is the work on the feasibility of space tourism by Patrick Collins, of University of Tokyo Research Centre for Advanced Science and Technology. The Japanese Rocket Society, with support from major industrial organizations, is planning a 50 - passenger, vertical take off and landing, single stage to orbit rocket - the "Kankoh-maru" - with a gross lift off mass of approx 550 tonnes (Isozaki et al, 1994). Their cost target is to get down to $\$ 200 / \mathrm{kg}$, believing that at this cost, the space tourism market will grow rapidly to several billion dollars per year, and require hotels in orbit, to cater for 10000 person accommodation after some years (Collins et al., 1994; Collins \& Isozaki, 1996). 
Thus there will be a market for construction metal, and for both stationkeeping and deorbit propellant.

\subsubsection{The potential future market for mass-in-orbit}

As a result of the activities described above, it is possible to hypothesize a conceptual post-2010 market for mass in low-earth-orbit (i.e. metals for construction, propellants, ceramics, life-support volatiles, and unprocessed mass for ballast and shielding against cosmic radiation). It is thought that the main early demand for mass in orbit will be for volatiles for propellant, closely followed by nickel-iron for construction material. The size and rate of development of this future in-orbit market for materials is not clear at the moment, but could easily exceed 1000 tonnes per year by 2010 , growing exponentially to tens of thousands of tonnes per year if any of the larger-scale activities "take off".

All these developments are presently stalled by the cripplingly high "airfreight" cost to lift anything into orbit. Present launch systems cost from $\$ 10,000$ to $\$ 20,000$ per kilogram to place mass in low earth orbit (LEO - 300km altitude), and about $\$ 40,000$ per kilogram to place mass in high geosynchronous earth orbit (GEO - 35,000km altitude). (Figure 1.1 on next page, from Stuart \& Gleave, 1991).

This high launch cost is the result of the "throwaway" nature of present launch vehicles, low flight frequency and utilization, and extreme manpower demands of present operations. Totally reuseable, low maintenance, fast turnaround, high utilization airline style vehicles and operations would probably reduce cost to launch by a couple of orders of magnitude, to something like $\$ 500$ per kilogram to low earth orbit, still a rather high figure. As noted above, market studies suggest that at about $\$ 200 / \mathrm{kg}$ there will be a multi-billion dollar space transportation market, driven initially by tourism. Large reduction in launch cost will prompt a huge growth in the market.

Thus, space commercialization is presently held back by the high entry cost, and, conversely, the development of low cost launch systems will be slow until there is an indication of a growing demand for transportation into space. 


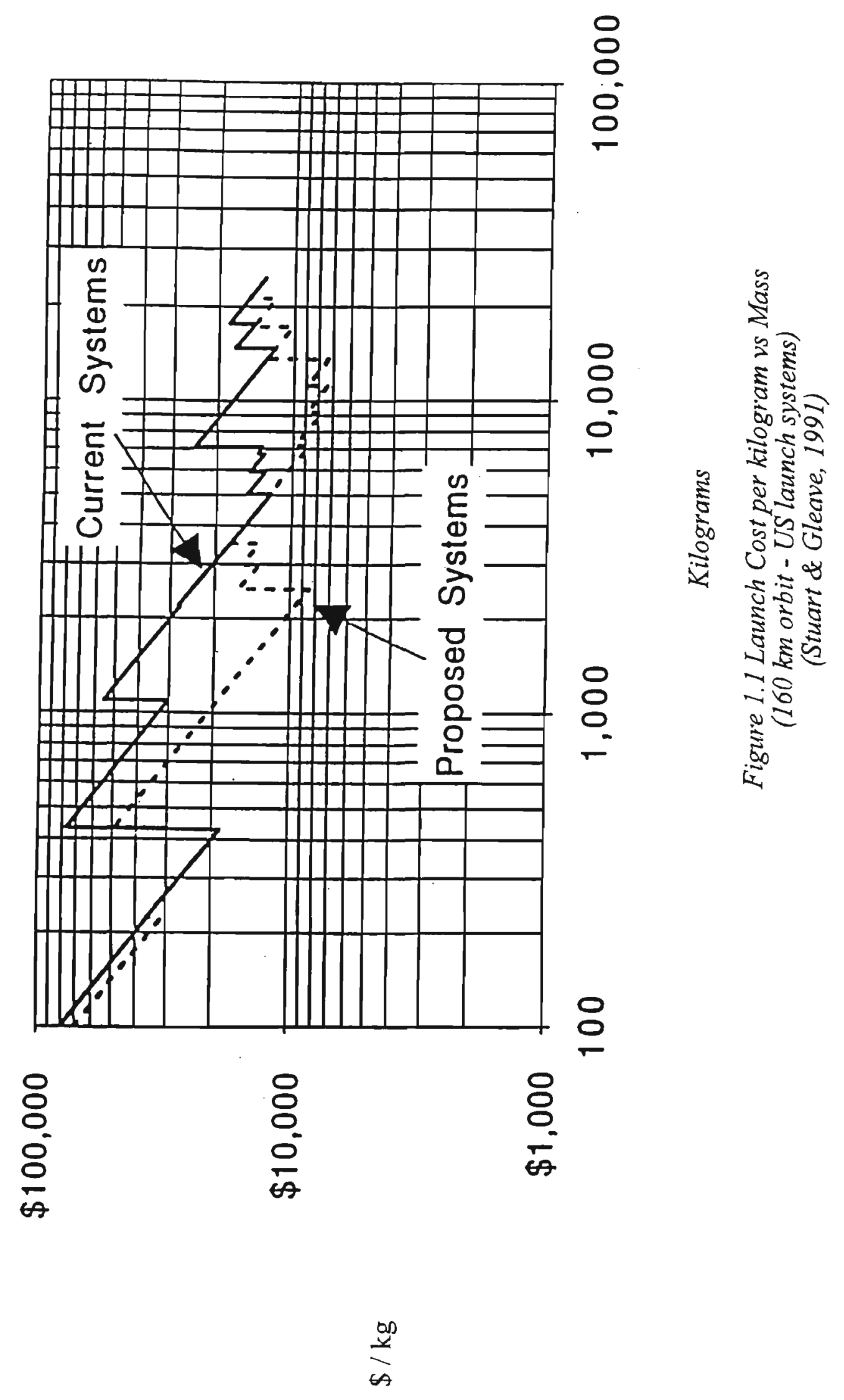




\subsubsection{The Accessibility and Competitiveness of Non-Terrestrial Resources}

Activities in orbit will require mass in orbit: metals for structural materials, oxygen, hydrogen, hydrocarbons, and ammonium compounds for fuels, and nonmetals for insulation and radiation shielding.

There are natural resources in space: metallic nickel-iron alloy, ilmenite, silicate minerals, hydrated minerals, bituminous material, and various volatiles, including water, ammonia, carbon dioxide, methane, and others. These have been identified either in meteorites, in lunar soil, or spectroscopically in asteroids and comets. There is also nonstop solar power at the rate of about $1.3 \mathrm{~kW} / \mathrm{sq} \mathrm{m}$ (thermal) or $100 \mathrm{~W} / \mathrm{sq} . \mathrm{m}$ electrical.

The idea of mining the asteroids was first discussed in concept by Cox and Cole, 1964, and in detail by Brian O'Leary in "Mining the Apollo and Amor Asteroids", in 1977. Other early writers are Herrick (1979), Morrison \& Niehoff (1979), and Kuck (1979).

Any industrial development in space requiring more than about a thousand tonnes of structural mass or propellant per year will direct attention to these materials as ores, in the true mining engineering sense.

Raw materials retrieved from non terrestrial sources need not attract the high "airfreight" costs referred to above. This is because the energy requirement to return material from many of these possible target asteroids is much less than the energy requirement to launch from Earth.

In addition, the freedom to deliver the velocity change non-impulsively, over an extended period, means that low power propulsion systems may be considered, and this opens up the possibility of choosing a system that uses solar power and derives its return-journey propellant from the target body.

This in situ propellant production then allows a high Mass Payback Ratio (mass multiplication). 
Thus there will potentially exist a profit-making opportunity for a resource developer who could develop a capability to recover space-based materials and return them for sale in low-earth-orbit, to capture the developing in-orbit market at its inception.

Accessibility of Space Resources: In space, the parameter which determines how easy or difficult it is to deliver mass from one orbit to another, is not distance, but is the required velocity change, $\Delta v$, needed to perform the transfer.

From consideration of velocity increments for different transfers, it can be seen that it is easier to go from low earth orbit (LEO) to nearly anywhere in the inner solar system than it is to get into orbit from the earth's surface (see table 1.1 below).

Table 1.1 Mission Velocity Requirements ( $\Delta v)$

$\begin{array}{lr}\text { Earth surface to LEO } & 8.0 \mathrm{~km} / \mathrm{s} \\ \text { Earth surface to escape velocity } & 11.2 \mathrm{~km} / \mathrm{s} \\ \text { Earth surface to GEO } & 11.8 \mathrm{~km} / \mathrm{s} \\ \text { LEO to escape velocity } & 3.2 \mathrm{~km} / \mathrm{s} \\ \text { LEO to Mars or Venus transfer orbit } & 3.7 \mathrm{~km} / \mathrm{s} \\ \text { LEO to GEO } & 3.5 \mathrm{~km} / \mathrm{s} \\ \text { LEO to HEEO } & 2.5 \mathrm{~km} / \mathrm{s} \\ \text { LEO to Moon landing } & 6.3 \mathrm{~km} / \mathrm{s} \\ \text { LEO to Near Earth Asteroid } & 5.5 \mathrm{~km} / \mathrm{s} \\ \text { Lunar surface to LEO (with aerobraking) } & 2.4 \mathrm{~km} / \mathrm{s} \\ \text { NEA orbits to Earth transfer } & 1.0 \mathrm{~km} / \mathrm{s} \\ \text { Phobos / Deimos to LEO } & 8.0 \mathrm{~km} / \mathrm{s}\end{array}$

Likely low $\Delta \mathrm{v}$ targets for initial resource development are the "Earth-Approaching" Apollo, Amor, or Aten type asteroids; the moons of Mars, Phobos and Deimos; the asteroid 1990MB Eureka, which is a Mars Trojan; any hypothetical Earth-Trojan asteroid; any of the Earth-orbit-hugging "Arjunas"; and indeed Earth's Moon itself. (Trojan asteroids are bodies which share the same orbit as a major planet, are 
gravitationally trapped in that orbit, and lead or lag the planet by 60 degrees in its orbit around the Sun.) These asteroid types are defined in Chapter 3.

\subsubsection{In-Situ Propellant Production}

The mission velocity $\Delta \mathrm{v}$ needed to reach these "near earth" low $\Delta \mathrm{v}$ targets is not

- significantly greater than that needed to place a communications satellite in geosynchronous earth orbit (GEO). The $\Delta \mathrm{v}$ required to return material from these targets is in some cases very much less than that required to lift mass into orbit from the surface of the earth, and it does not necessarily have to be provided impulsively. It can be imparted gradually, over several weeks, thus very substantially reducing the demands on the propulsion / power system.

If the return transfer can be accomplished using part of the retrieved non-terrestrial mass as reaction mass, or propellant, and solar energy for the power source, or onboard nuclear power, then it becomes possible to return to earth orbit very much more mass than the outbound-leg earth-orbit-departure mass of the mining-processing spacecraft. Mass multiplication factors above 100 are the initial aim.

The effect of the very low energy requirement to return some asteroidal material to earth orbit, together with the possibility of using asteroid-derived mass for propulsion, is that some asteroidal material may be able to be delivered into Earth orbit for a cost which is very much less than the cost to launch the same mass of material from the Earth.

\subsection{Purpose and Outline of Thesis}

The intent of this thesis is to identify the requirements that must be satisfied by an Earthapproaching asteroid or short-period comet to make it an "orebody" in the mining engineering sense: that is, to identify it as a resource source that can support an economic materials retrieval project.

These economic and technical requirements are: 
(i) a development of a market for the products

(ii) adequate spectral data to suggest the body will contain the desired materials

(iii) identification of the range of orbital parameters that give reasonable accessibility and mission duration

(iv) development of feasible retrieval concepts (via In-Situ Propellant Production)

(v) development of feasible concepts for mining \& processing

(vi) "adequate" Mass PayBack Ratio, and most importantly, positive Net Present

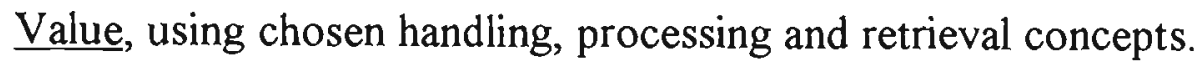

The following diagram is intended to show how these concepts interact in this thesis.

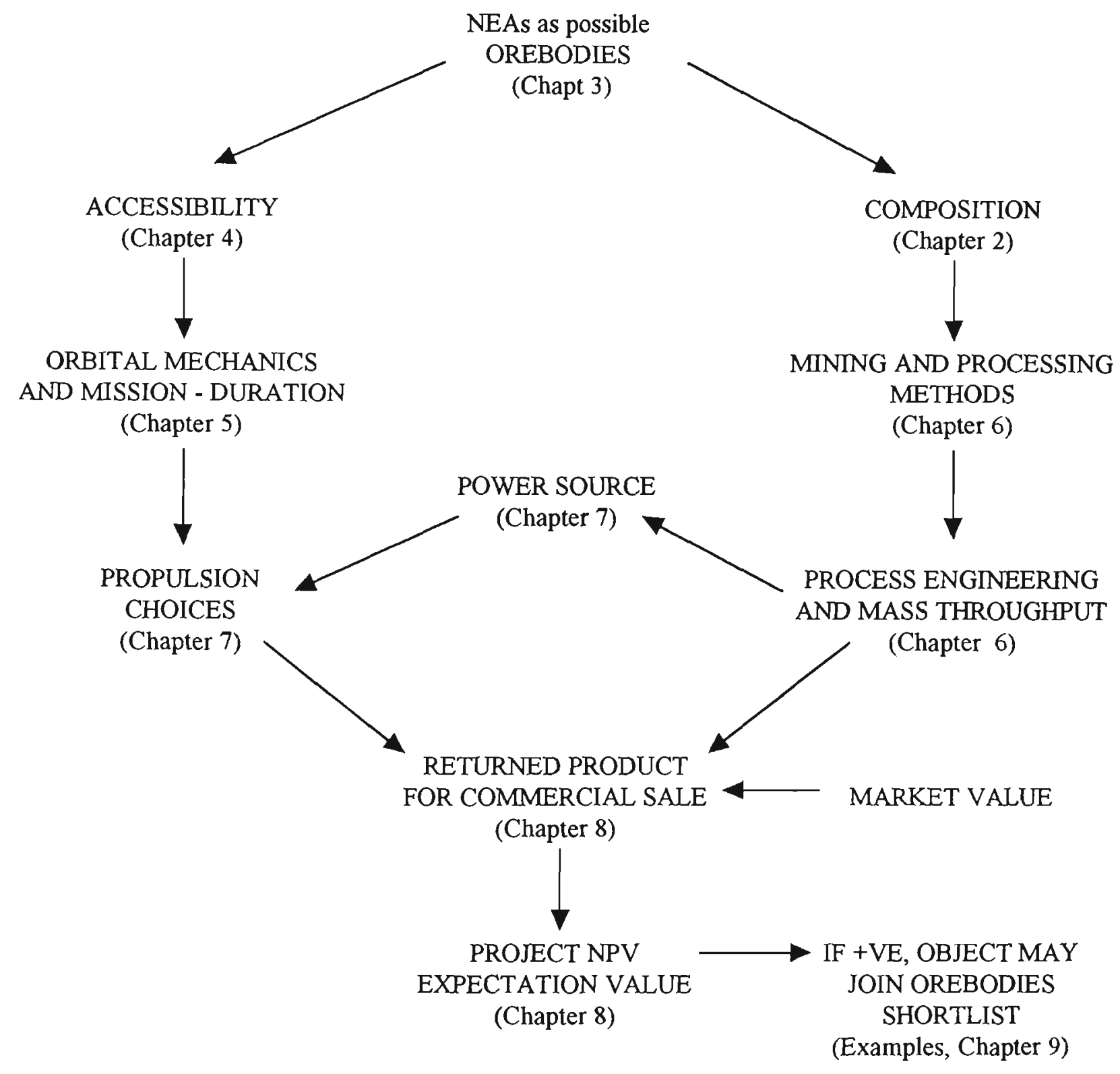

Figure 1.2 Concepts Flowchart 
The above flowchart is a simple description of the methodology which is developed in this thesis for determining the technical and economic feasibility of any hypothetical asteroid mining project. It also shows the dissection of the topics into their respective chapters.

A brief review of the Chapter contents follows.

\subsubsection{Review of Asteroid Geology \& Resources}

Astronomical work over the last fifteen years has increased the number of known Near Earth Asteroids (NEAs) from about 30 (Wetherill, 1979) to about 380 (see Asteroid Listings, page 195). Asteroid geology has also advanced dramatically in the last decade, drawing on spectroscopic and dynamical studies of asteroids and comets, and meteorite studies, and reasonable correlations can now be made between spectral / photometric asteroid types and inferred surface mineralogy.

It is now believed that many asteroids may be "volatiles bearing", containing clays, hydrated salts, and hydrocarbons. It has also become clear that there is a continuum from asteroidal to dormant cometary bodies, within the population of NEAs.

A subset of the NEAs is more accessible than the Moon, in terms of required mission velocity $(\Delta v)$ for outbound and return trips.

A matrix of alternative asteroid types and proposed products has been developed, from consideration of meteorite types and project options.

\begin{tabular}{|c|c|c|c|}
\hline $\begin{array}{l}\text { meteorite } \\
\text { mineralogies }\end{array}$ & $\begin{array}{l}\text { spectroscopically } \\
\text { inferred asteroid } \\
\text { geology }\end{array}$ & $\begin{array}{l}\text { possible mining } \\
\text { and processing } \\
\text { concepts }\end{array}$ & $\begin{array}{l}\text { desired } \\
\text { product }\end{array}$ \\
\hline
\end{tabular}


Table 1.2 Matrix of spectral type, inferred mineralogy, and potential products.

\begin{tabular}{|l|l|l|}
\hline Type & Inferred Mineralogy & Product \\
\hline $\mathrm{C}, \mathrm{D}, \mathrm{P}$ & clay, organics, ice at depth? & volatiles: $\mathrm{H}_{2} \mathrm{O}, \mathrm{CO}_{2}, \mathrm{CH}_{4}$ \\
$\mathrm{~B}, \mathrm{G}, \mathrm{F}$ & clay, silicate, ?limestone, & volatiles: Nickel-Iron metal \\
& ? Nickel-Iron metal & \\
Q, S, M & silicates, Nickel-Iron metal & metal, silicates, Platinum \\
& & Group Metals (PGMs) \\
\hline
\end{tabular}

In the case of those bodies which are dormant or extinct comets (there are several likely candidates), there may be remnant primordial ices within their cores, and hence possible sources of volatiles for future space industry.

Asteroid "geography": NEAs are classified by orbital parameters into Apollos, Amors, and Atens. The "Arjunas" are a group of small objects in very Earth-like orbits. The discovery rate of NEAs is now quite high, about 50 per year.

\subsubsection{Asteroid Accessibility and Mission Profiles}

Accessibility is defined in terms of $\Delta v$, total velocity change required, for outbound and for return trajectories. Approximately $10 \%$ of NEAs are more accessible than the Moon and at least $50 \%$ of these are likely to be potential orebodies.

There have been various concepts proposed for mining and retrieval to low-earth-orbit of materials from NEAs, but it has been noted in the literature (Cutler \& Hughes, 1985; Lewis Ramohalli \& Triffet, 1990; Ramohalli, Kirsch, \& Priess, 1994; Oxnevad, 1991) that means of comparison of mission concepts are not well-developed.

This thesis develops methods for comparison of different asteroid mining concepts, and for choosing between various trajectory, mission, and engineering alternatives, so as to maximize project economic feasibility.

Application of the concepts of celestial mechanics show that 
(i) simple estimates of "global minimum" delta-v can be made, by several methods;

(ii) the launch windows for these "global minimum" opportunities are infrequent, but somewhat more expensive local minima occur at approx 2-yearly intervals, for most NEAs;

(iii) good outbound opportunities do not generally fit with good return opportunities;

(iv) long synodic periods and phasing requirements militate against multiple-return mining missions;

(v) Earth-return hyperbolic velocity should be kept low (lunar flyby capture may be useful).

(vi) high-e targets require Hohmann transfers out and back, and a short mining season at aphelion;

(vii) low-e targets may use continuous-thrusting propulsion, and extended mining season.

(viii) missions appear to be classifiable by trajectory type and linkage with target type

Missions appear to be classifiable into 5 distinct types.

Despite the above constraints, there is a growing subset of targets that appear to be intermittently accessible for an outbound $\Delta v$ of under $6 \mathrm{~km} / \mathrm{s}$, and offering return departure $\Delta \mathrm{v}$ under $2 \mathrm{~km} / \mathrm{sec}$.

\subsubsection{Concepts for Mining, Processing, Power, and Propulsion}

Requirements and engineering choices for mining and processing depend on the assumed regolith mineralogy and bulk handling properties, and on the assumed subsurface composition and properties, if the desired material is to be recovered by drilling. Process options are:

\footnotetext{
in-situ fluidization

mechanical collection and thermal dehydration

mechanical collection and magnetic separation

carbonyl process.
} 
Table 1.3 Possible Products and Sources

\begin{tabular}{|c|c|c|}
\hline \multirow{2}{*}{ Type } & \multicolumn{2}{|c|}{ Product and Process } \\
\cline { 2 - 3 } & Volatiles & Metal \\
\hline "cryptocometary" & $\begin{array}{c}\mathrm{H}_{2} \mathrm{O}, \mathrm{CO}_{2} \\
\text { in-situ fluidization } \\
\text { carbonaceous }\end{array}$ & $\mathrm{H}_{2} \mathrm{O}, \mathrm{CO}_{2}$ \\
thermal dehydration & ------ & $\mathrm{NiFe}$ \\
ordinary chondrite & magnetic separation \\
metallic & $-\cdots$ & NiFe \\
& & magnetic separation \\
& & NiFe, Platinum Group Metals \\
& & carbonyl process \\
\hline
\end{tabular}

For reasons of simplicity, risk minimisation, and near-term achievability, as well as achievability of economic viability, various choices would have to be made, and this thesis will expand only on some of these in further detail rather than all alternatives:

Product and Engineering Choices (those to be expanded on are underlined):

product

water; metal; other gases; silicates; PGMS

process

drilling with insitu melting and extraction;

electrostatic / magnetic extraction; carbonyl

extraction; heat, volatilize, and condense

target type : overtly cometary; extinct or dormant comet; overtly carbonaceous or hydrous asteroid; S-type asteroid; overtly metallic asteroid.

trajectory and

mission type

power

propulsion

control
"Apollo type"; "Aten- type"; "Arjuna type"; "Amor type"

SP comet type; low-e, plane-change-dominated.

solar thermal; nuclear thermal; photovoltaic;

mass driver; arcjet; steam rocket

manual : telepresence; machine autonomy

Propulsion and power choices are linked; only a subset is technically viable. In-situ production at the asteroid of the propellant required for materials return is an 
important "enabling" concept. Sensible system choices are given below and further discussed in Chapter 7.

Table 1.4 Propulsion and Power Choices

\begin{tabular}{|c|c|c|c|c|}
\hline & & \multicolumn{3}{|c|}{ Propulsion } \\
\hline & & steam rocket & arcjet & mass driver \\
\hline \multirow{3}{*}{ Power } & solar thermal & Yes & No & No \\
\hline & solar PV & No & Yes & Yes \\
\hline & nuclear & Yes & Yes & Yes \\
\hline
\end{tabular}

\subsubsection{Project Feasibility and Selection Criteria}

The intent of this study is to develop a robust approach to defining the selection criteria which will determine the choice of preferred prospective target orebodies within the population of Near-Earth-Objects. It will be necessary to develop a logical way of comparing competing project concepts; ie a "Figure of Merit" for comparing asteroid mining concepts. Most of the (relatively fragmentary) literature to date has concentrated on Mass Payback Ratio, but it is clear that Net Present Value (NPV) is a more basic criterion for determining economic viability. The formula for NPV can be readily expanded so as to explicitly reference the astrodynamical parameters that define various asteroid missions.

The concepts discussed here involve the design for the simplest, minimum mass and cost product return system possible, which precludes a crewed mission. It assumes a remote controlled or automated mining and processing plant and assumes initial in-orbit market will be for volatiles for fuel. 


\subsection{Conclusions}

This thesis provides the outline, plan, or generic methodology for performing Feasibility Studies for the asteroid mining projects of the early years of the next century. 


\section{Chapter 2: Asteroidal Resources}

\subsection{Attractiveness of the Near-Earth Asteroids versus other targets.}

This Section reviews the Near-Earth Asteroids as potential resource sources in comparison with the other more-generally proposed alternatives, which are the Moon; and Phobos or Deimos, the moons of Mars.

\subsubsection{The Moon}

The Moon's relatively deep gravity well calls for an impulsive (i.e. rapidly applied) velocity change of approx $1 \mathrm{~km} / \mathrm{sec}$ to transfer from lunar orbit to lunar surface or vice versa; and approx $2.4 \mathrm{~km} / \mathrm{sec}$ either to perform a soft landing from a direct-from-earth trajectory or to take off on a direct to LEO (low Earth orbit) trajectory. The orbit to surface and surface to orbit transfer impulse requirements demand a large thrust-to-mass ratio rocket motor. This is a severe constraint on propulsion system and on propellant type; it means that the rocket must be either chemical or nuclear thermal, and the propellants of choice either hydrogen and oxygen, or hydrogen, respectively.

Electromagnetic launchers are not a near-term option because their emplacement demands very high level engineering capability and significant on-surface infrastructure. In addition, their inflexibility in launch azimuth and ultra-high g-force regime renders them much less useful than other launch systems.

Although there are simple schemes available for extraction of oxygen from lunar soils, there are no known sources of water or hydrogen on the moon other than gases from the solar wind trapped in the top layer of the regolith, present in ppm concentrations. The absence of hydrogen on the Moon means that as a source of resources the Moon is a "non-starter", unless lunar polar ice deposits are proven, in which case the economics of activity on the Moon would be profoundly changed. 
There is a possibility of permanent deposits of water ice and other ices in perpetually shadowed craters near the north and south poles; this has not yet been properly investigated. The recent Clementine mission revealed the giant Aitken - South Polar Basin within which such ice deposits may exist, and a radio-reflection experiment done during the mission gave a strong depolarization signal in a small area centred over the South Pole that is characteristic of water ice.

If substantial water ice deposits do exist, the Moon would then become a very easy place to get to and return from, despite its gravity well, because of the insitu availability of hydrogen and oxygen for fuel, and a very easy place to colonize.

It must be noted however that the Moon is the only non-terrestrial body humans have actually visited; there is certain knowledge of the composition of the soil at some dozen locations on its surface. It is known with certainty how the soil might be processed to recover free metal (Ni-Fe) fines (Agosto, 1981), or iron and oxygen from its ilmenite, or aluminium and oxygen from the feldspar. In addition, solar power is available, and beamed power may be an exportable commodity, provided solar cells can be manufactured on-site.

The relatively high gravity means that structure design and materials handling will not be dissimilar to earth based solutions; stable foundations and gravity imply easy civil engineering; there is an extensive literature regarding possible mining techniques for the Moon (see e.g. US Army Corps of Engineers, 1990).

An important advantage of the Moon over other targets is that communications are nearly instantaneous, and therefore real-time remote control from Earth is possible. However, any hypothetical polar ice mining will need several communications satellites in lunar polar orbit.

In summary, the arguments against the Moon as a source of resources include: no volatiles, hardly any free metal, very difficult to extract any useful product (with the exception of oxygen from ilmenite); two weeks of imposed darkness out of every four, 
hence need for energy storage of heroic proportions, or for $50 \%$ downtime, or for nuclear power; also very severe temperature cycling. There is however a small spot near the lunar South Pole which appears to have near permanent sunlight.

\subsubsection{The moons of Mars}

Phobos and Deimos are potential resource targets. There is already high resolution photography of their surfaces, showing a well developed soil or regolith layer; and they are thought to be captured D-type asteroids. If this is so, they may contain both recoverable volatiles and easily extractable metal. The Russian Phobos 2 probe detected a shock front near Deimos, indicating either a magnetic field or evidence of outgassing (pers. comm., D. Kuck, 1996). However, spectral signatures for hydrated silicates have not been seen. It has been calculated (Fanale \& Salvail, 1990) that deep primordial ice may persist in Phobos and Deimos, at depths of (approx) 20 metres (polar) to 100 metres (equatorial). This being so, these moons could be highly prospective as ice orebodies.

The velocity change needed to rendezvous with these minor planets is not great. It is the same as that needed to launch out of earth orbit onto a Mars transfer ellipse, i.e. approx $3.5 \mathrm{~km} / \mathrm{s}$, plus the $\Delta \mathrm{v}$ to circularize at the satellite's altitude. This assumes a zero-fuelrequirement aerocapture at Mars to match with Mars's heliocentric velocity, and to drop into an elliptical orbit with its highest point at the altitude of the target satellite. The satellite chosen should be Deimos because it is the higher, and hence a smaller circularization velocity will be required $(700 \mathrm{~m} / \mathrm{s})$. Also, the velocity required for the later launch of valuable material back to earth orbit will be less than would be the case if it were to be launched from Phobos.

Phobos and Deimos are natural bases from which to conduct manned exploration and colonization of Mars.

There is a very significant drawback with the choice of the moons of Mars, however. This is that the navigational demands for successful aerocapture by close fly-through of the Mars atmosphere are extreme, and are not demonstrated to be within the present state of the art. Spacecraft control during aerocapture must necessarily be autonomous, 
because telemetry time-of-flight time delay is many minutes, longer in fact than the entire aerocapture manoeuvre.

In the absence of aerocapture, an additional $\Delta v$ of about $3.5 \mathrm{~km} / \mathrm{s}$ is needed to kill hyperbolic velocity and drop into Mars orbit. In addition, the post-Mars-capture $\Delta v$ needed to circularize at Deimos of $700 \mathrm{~m} / \mathrm{sec}$ is similar to or greater than the total deep space $\Delta \mathrm{v}$ for certain Apollo asteroid rendezvous manoeuvres. Additionally, some 2 tonnes of chemical fuel would be needed to lift a 1000 tonne payload off Deimos (assumes fuel $\mathrm{I}_{\mathrm{sp}}-$ Specific Impulse- of $270 \mathrm{sec}$ ).

\subsubsection{The Near-Earth Asteroids}

The Apollo, Amor, and Aten asteroids are the three classes of Earth-approaching asteroids or Near Earth Asteroids. The NEAs are quite small objects, generally about 1 $\mathrm{km}$ in diameter, and may be metallic, silicate, or "primitive" carbonaceous chondrite, inwhich case, water-bearing, i.e., hydrated in composition.

Apollos are asteroids which cross the Earth's orbit; they do represent a collision threat, and are now known to be the origin of some of the regular named meteor showers (Morrison (1993), Olssen-Steel (1987)). Amors do not cross Earth's orbit, but approach it to within 0.3 Astronomical Units (AU). Atens are Earth crossers which have the larger part of their orbit within Earth's orbit, i.e., their orbital semimajor axis is less than that of the Earth's orbit. Atens therefore also represent a collision threat.

The mission velocity to depart LEO for the most accessible of the Apollo-Amor-Aten asteroids is of the order of 5 to $6 \mathrm{~km} / \mathrm{s}$, with a 'deep space' rendezvous (orbit matching) velocity increment of $1 \mathrm{~km} / \mathrm{s}$ or less for the most favourable outbound trajectories. The required velocity for departure to return to Earth is generally small, also of the order of 1 $\mathrm{km} / \mathrm{s}$, and it can be imparted slowly, over a period of many months if necessary, and hence is suited to a propulsion system that is power-limited but continuous, and has available to it a large amount of reaction mass. 
Table 2.1 Targets Comparison Matrix

\begin{tabular}{|l|l|l|l|l|}
\hline target & \multicolumn{1}{|c|}{$\begin{array}{c}\text { propulsion } \\
\text { requirements }\end{array}$} & resource type & $\begin{array}{l}\text { timetabling } \\
\text { constraints }\end{array}$ & \multicolumn{1}{c|}{ comments } \\
\hline Moon & $\begin{array}{l}6.3 \mathrm{~km} / \mathrm{s} \text { out; } 2.4 \\
\mathrm{~km} / \mathrm{s} \text { back }\end{array}$ & $\begin{array}{l}\text { silicates giving } \\
\text { aluminium and } \\
\text { oxygen }\end{array}$ & none & $\begin{array}{l}\text { both out and back } \\
\text { must be impulsive, } \\
\text { high thrust }\end{array}$ \\
\hline $\begin{array}{l}\text { Phobos } \\
\text { or } \\
\text { Deimos }\end{array}$ & $\begin{array}{l}\sim 4 \mathrm{~km} / \mathrm{s} \text { out, } 3 \mathrm{~km} / \mathrm{s} \\
\text { back; using } \\
\text { aerobraking at Mars } \\
\sim 8 \mathrm{~km} / \mathrm{s} \text { out without } \\
\text { aerobraking at Mars }\end{array}$ & $\begin{array}{l}\text { silicates, } \\
\text { possibly NiFe, } \\
\text { volatiles, deep } \\
\text { buried ice }\end{array}$ & $\begin{array}{l}\text { 2 year } \\
\text { synodic } \\
\text { period }\end{array}$ & $\begin{array}{l}\text { lunar gravity assist } \\
\text { may be used both } \\
\text { out and back; } \\
\text { Mars aerocapture } \\
\text { needs autonomous } \\
\text { guidance }\end{array}$ \\
\hline $\begin{array}{l}\text { subset of } \\
\text { NEAs }\end{array}$ & $\begin{array}{l}5 \mathrm{~km} / \mathrm{s} \text { out, } 1 \mathrm{~km} / \mathrm{s} \\
\text { back; low thrust } \\
\text { non impulsive } \\
\text { return acceptable }\end{array}$ & $\begin{array}{l}\text { water, metal, } \\
\text { carbon dioxide, } \\
\text { silicates }\end{array}$ & $\begin{array}{l}\text { irregular, } \\
\text { imp is } \\
\text { important } \\
\text { constraint }\end{array}$ & $\begin{array}{l}\text { lunar gravity assist } \\
\text { may help } \\
\text { especially on } \\
\text { return }\end{array}$ \\
\hline
\end{tabular}

The propulsion needs for a subset of NEAs are therefore much less than for either the Moon or for Phobos/Deimos.

Thus some NEAs are very easy to reach and to return from; they appear to contain a wide range of potential resources (not all necessarily in one body); it is becoming evident that a total gradation exists between comets and asteroids; solar power is available; onasteroid production of return propellant is possible, which enhances (by orders of magnitude) the potential mass return; it is known that at least some asteroids have regoliths; metallurgical recovery of metals and volatiles will clearly be much easier than from lunar soils, because of the very much higher grades in asteroidal regoliths.

Note that a number of $\mathrm{E}$ and $\mathrm{M}$ type asteroids, previously thought to be anhydrous, are now shown to have spectral indications of hydrated silicates: this greatly enhances their potential value as resource objects (Williams \&Tedesco, 1995). Platinum Group Metals are valuable enough to return to Earth for sale (Kargel, 1994), albeit in the case of near term and small scale operations, probably only as a byproduct.

There are however several serious technical challenges associated with remote-controlled operations on the asteroids. One is that the radio signal time of flight may be anything 
from 5 to 30 minutes; thus a remote miner must be designed to be "smart"; it must use machine intelligence to operate semi autonomously, with only high-level human operator support.

A second problem is that we do not know how to land on, or more correctly, dock with, a slowly rotating asteroid (average rotation period about 6 hours) the gravity of which is 0.001 of Earth's, or less. There is also the necessity to secure equipment during mining operations, against various reaction forces; and there is the need to design processes which will run in zero-gravity.

The third major problem is that there is at present only inadequate geological knowledge; and detailed spectra exist for only very few of the NEAs. This can be easily addressed by support for low-cost astronomical studies.

A further interesting observation is that as mining projects, the asteroids appear to be "one season" mines, because of synodic or phasing constraints on launch and return arrival times.

\subsubsection{The Resource Attractiveness of the NEAs:}

Despite these drawbacks, i.e., 'one-season' mines, inadequate present geological knowledge, and necessity to develop autonomous mining equipment, the NEAs still appear to contain the most attractive orebodies for supply of water or 'celestial stainless steel' to facilities in LEO. The purpose of this thesis is to define more clearly the requirements that need to be met to achieve technical and financial feasibility, for any hypothetical asteroid mining project.

"... the Near Earth Asteroids are compositionally diverse, ... with $\mathrm{km}$-sized chunk of natural stainless steel, the cores (possibly ice-rich) of extinct comets, primitive unmelted planetary materials, and differentiated rocks similar to lunar basalts....". (There are also, it is now known, asteroids containing water-bearing clays and carbonaceous oil-shalelike materials ...) 
"The panoply of materials is vastly broader and richer than those known to be present on the Moon. At least one-fifth of NEAs are volatile-rich, ... and almost all the others are metal-rich. Also, about one fifth are energetically more accessible than the surface of the Moon .....Schemes are already known by which spacecraft dispatched on round-trip missions to the best of these asteroids could return over 100 times their own mass of asteroidal resources to near-earth-space" -Lewis and Hutson, 1993.

Thus, the asteroids may be a veritable cornucopia of resources for the industrialization of space. The NEAs are good -probably the best- prospects for early extraterrestrial resource recovery ventures.

\subsection{Asteroidal and Cometary Geology and Mineralogy}

The geology and mineralogy of asteroids can only be interpreted from visible and $\mathbb{R}$ spectral studies by astronomers, by attempting to correlate information from meteorite samples, from photographs taken during the two asteroid flybys of Gaspra and Ida by the Galileo probe, and radar studies using radio telescopes.

Asteroids Gaspra and Ida, both S-types, photographed by Galileo during its flybys of them in 1991 and 1993 respectively, both show a well developed regolith despite the negligible gravity (Sullivan et al, 1995). There had been speculation that asteroids would necessarily lose any fines and be reduced to bare rock or metal. Ida was found to have a moon, named Dactyl, which, despite being only $1.5 \mathrm{~km}$ in diameter, itself retains a regolith.

Two Apollo asteroids, $1986 \mathrm{DA}$ and 3554 Amun, are known from radar reflection studies to be solid metal (Ostro et al, 1991). Others are suspected to be so from spectroscopic studies.

There are radar images of only three asteroids, Castalia and Toutatis, which both appear to be contact binaries, and very irregular, and Geographos, which is "the most elongated body in the solar system" (Ostro et al., 1995). See images on page 24. 
Photometric and spectroscopic studies appear to show a wide variety of compositions, including metallic nickel-iron, silicates, hydrated silicates, and bituminous.

All of our assumptions about the makeup of asteroids depend on spectroscopic and photometric information and on implied linkages with meteorites. Meteorites are the only "ground truth" available, and selection biasses are large and not well known. For example, not all lumps of material entering the upper atmosphere will survive to reach the ground: volatile and structurally weak or friable objects will generally not survive to end up in museum display cases or meteoricists' laboratories. (An interesting exception is the recent recovery of an apparent ice meteorite in China! -Wang \& Zhang, 1995.)

There is also selection bias at work in asteroid discovery rates and proportions: darker asteroids are clearly more difficult ot discover than lighter asteroids.

Thus in order to review asteroid taxonomy and assumed mineralogy, meteorite mineralogy and taxonomy must first be discussed. 


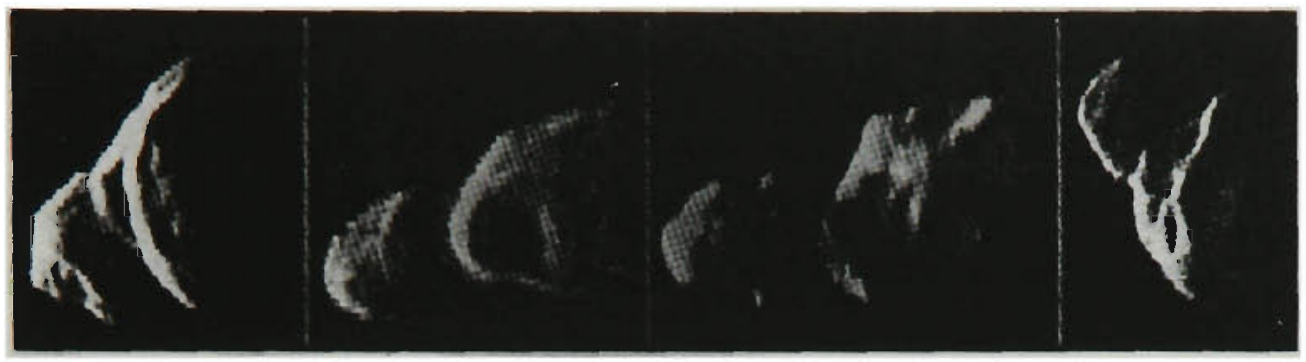

\section{Figure 2.1}

\section{Near-Earth Asteroid}

Toutatis (from JPL)

Figure 2.2

NEA 1620 Geographos (from Ostro et al)

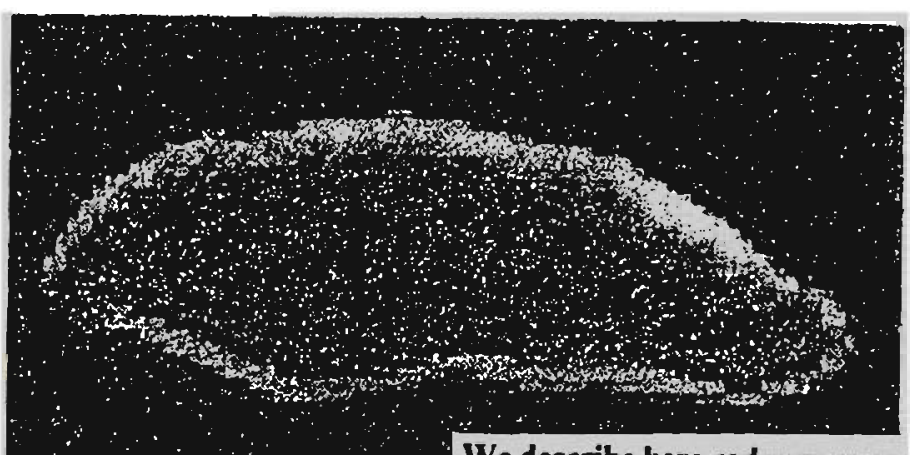

We describe here radar measurements of the Earth-crossing asteroid 1620 Geographos during its recent close encounter with the Earth. We have determined the silhovette of Geographos along its rotation axis, and confirm earlier lightcurve-based conjectures ${ }^{10}$ that this object has a very unusual shape.

Figure 2.3

Comet $p /$ Halley

(from Max-Planck Institut)

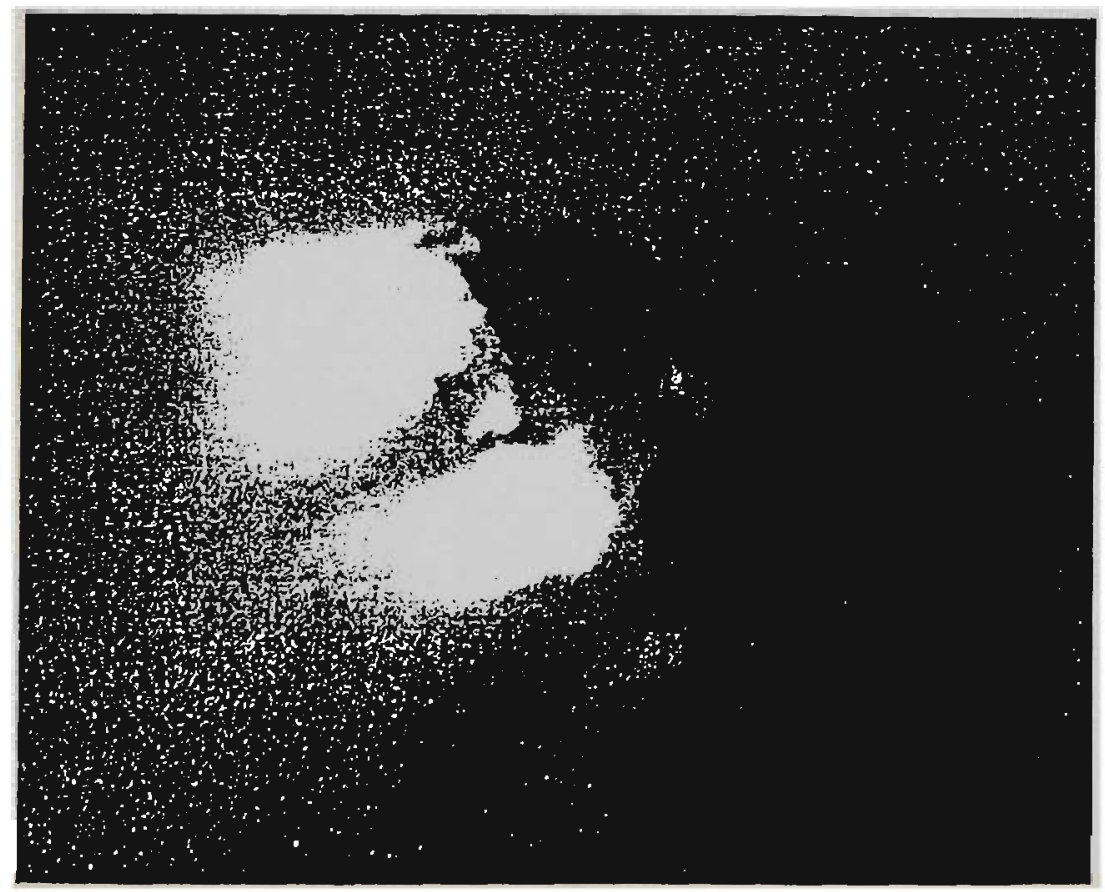

Composite photograph of the nucleus of comet Halley as recorded by the Giotto spacecraft March 14. 1986. The Sun is at left. The spacecraft approached the nucleus from the dark side. and is viewing the sunrise terminalor. Discreie active areas and dust jets are visible on the sunlit hemisphere. The dark. irregular nucleus is silhouetted against the bright dust coma of the comet. The nucleus is approximately $16 \mathrm{~km}$ long by $8 \mathrm{~km}$ wide. Ploto councsy of H. U. Kefler. Max-Planck-Institut für Acronomic. Linklau. 


\subsubsection{Meteorite Mineralogy and Taxonomy}

Meteorites are classified into Stones, Stony-Irons, and Irons.

The Stones comprise two subclasses, the chondrites and the achondrites. The Chondrites appear to be samples of primitive solar system accretion bodies with zero to low metamorphism, possibly with some aqueous alteration, and possibly some metasomatism. These are believed to be "never melted" bodies, dating from the origins of the solar system, and to be compositionally unchanged since their condensation from the presolar nebula, with composition correlating with mean radius of formation from the Sun. These are further subdivided into Enstatites, Ordinary Chondrites (these contain "abundant" free metal), and Carbonaceous Chondrites (these contain "no free metal, but autoreduction of magnetite and other iron oxides by carbon permits extraction of up to $40 \%$ by weight of total HCNO volatiles" - Lewis and Hutson, 1993).

The second classification within the Stones, the Achondrites, are igneous, basaltic, and show clear evidence of having been differentiated from a melt.

The Stony irons and the Irons are even more heavily differentiated and are interpreted to be fragments from the metallic core or the mantle-core boundary of an igneously differentiated planetesimal which suffered disruption in a cataclysmic impact. 
Table 2.2 Meteorite Taxonomy

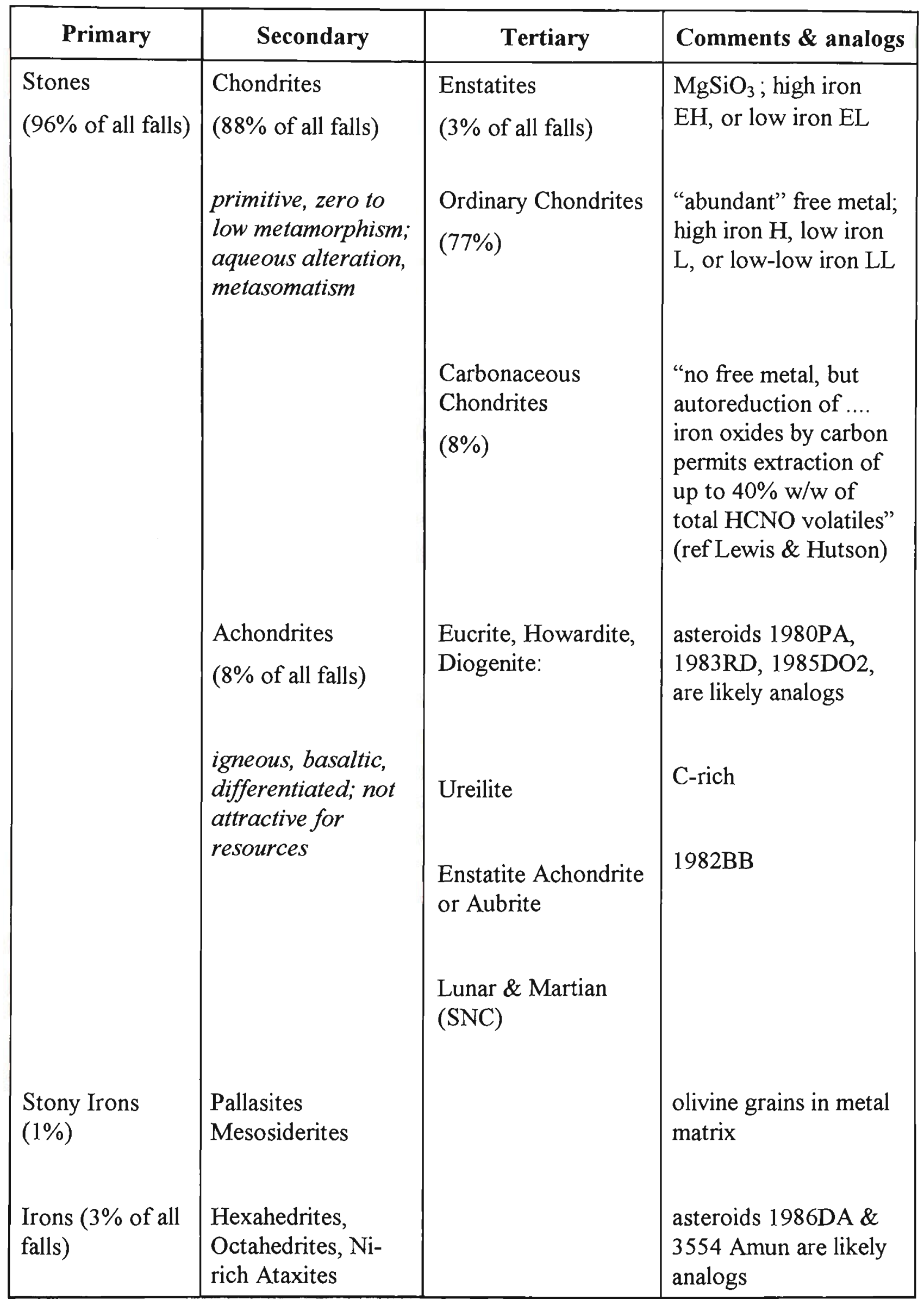




\section{Ordinary Chondrites}

The identity of the source asteroids for Ordinary Chondrites is a major puzzle of meteorite/ asteroid studies, because despite their abundance in meteorite collections, the only spectral matches are apparently to 1864 Apollo and to 3628 Boznemcova (Bell, 1995).

Theories and possibilities are:

1. Some S-type asteroids may be source objects for Ordinary Chondrites but regolith weathering processes may have enhanced the metal content of the surface and hence reddened their spectra. However Clark, Fanale, and Salisbury, 1992, argue convincingly against this hypothesis in "Meteorite - Asteroid Spectral Comparison - the Effects of Comminution, Melting, and Recrystallization".

2. Ordinary chondrite source bodies may have all attritioned to sizes too small to detect by telescope.

3. Regolith processes (gardening, radiation exposure) may have darkened the surfaces of the origin bodies to the point that they now have quite different spectra and get identified (erroneously) as C-type asteroids.

4. Ordinary chondrites may come not from asteroids but from comets.

5. Maybe all come from Boznemcova, which is close to Kirkwood gap, and has matching spectrum.

6. It has been suggested that commentators are mistaken in applying a time - constant "Uniformitarianist" model to the type-distribution information, and the proportions of 'falls' do in fact change over time. "There is no reason why we should expect meteorite type-abundances to be in line with source population abundances, over intervals of less than (say) 10 Myrs." (Gaffey, 1995) -present author's italics.

The implication of this conclusion for asteroid mining is that the spectroscopic evidence suggests a much higher abundance of volatiles in asteroids than might be interpreted from the falls proportion for carbonaceous chondrites in the total recovered meteorite population. If the spectroscopic evidence is more reliable, and in particular taking into 
account the observational bias against easy detection of $\mathrm{C}, \mathrm{D}$ class asteroids, then there may be much greater availability of volatiles orebodies in space than meteorite falls indicate.

\section{Carbonaceous Chondrites}

Carbonaceous chondrites give the only 'ground truth' there is for composition of the most likely asteroidal source for volatiles, the C, D, P, F, and G class asteroids. The Carbonaceous Chondrite classifications are: $\mathrm{CI}, \mathrm{CM}, \mathrm{CO}, \mathrm{CV}, \mathrm{CR}$.

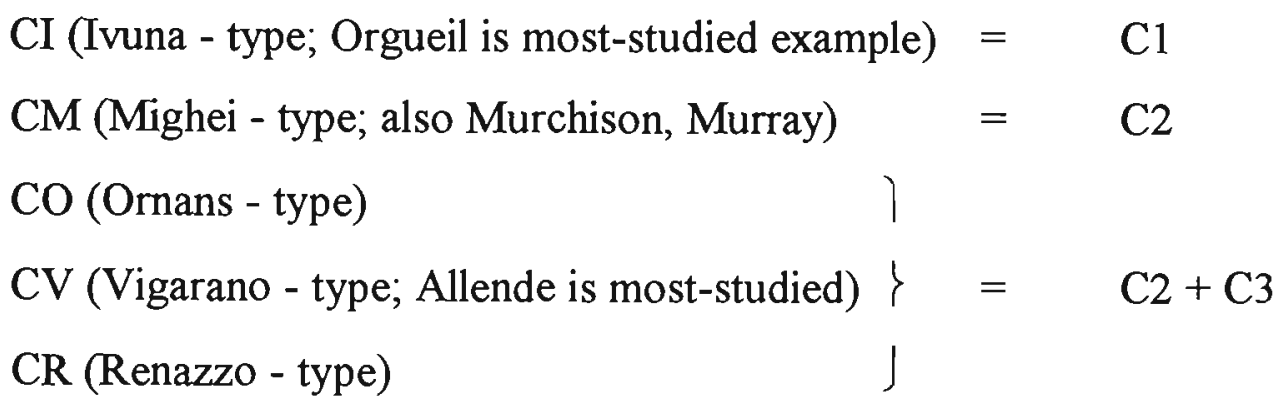

The numbers refer to a petrological scale, ranging from 1 to 6 , where $1=$ maximum aqueous alteration (e.g. to hydrated salts or clays); $3=$ unaltered (most primitive texturally), and very friable; and $6=$ maximum thermal alteration (well cemented; diagenesis); chondrules totally absorbed.

There is an implicit identification of C-type asteroids with meteorites of classes $\mathrm{C} 1 / \mathrm{C} 2$ (being relatively pristine chemically -but not texturally- with high proportion of clays) and with meteorites of classes $\mathrm{C} 3 / \mathrm{C} 4$, which exhibit some thermal and aqueous alteration, metasomatism, and metamorphism. All such "identifications" must be treated with suspicion and care.

Note that $\sim 70 \%$ of the carbon in CM-type carbonaceous chondrites is present as "insoluble, macromolecular" material. This material is described by Hayatsu 1977, 1980 thus: "Macromolecular carbon (in carbonaceous chondrites) is composed of condensed aromatic, heteroaromatic, and hydroaromatic ring systems in up to 4-ring clusters, crosslinked by short methylene chains, ethers, sulphides, and biphenyl groups". He notes its 
similarity with vitrinite macerals of low-volatile bituminous coals, or with type III kerogens from oil-shales. Cronin et al, 1988, state "macromolecular carbon with $\mathrm{H}, \mathrm{N}$, $\mathrm{O}$ and $\mathrm{S}$... has been called "kerogen-like" and an "organic polymer", but is different from both; it is acid resistant and in this regard fits the formal definition of kerogen ---". The stoichiometry has variously been given as

$\mathrm{C}_{100} \mathrm{H}_{71} \mathrm{~N}_{3} \mathrm{O}_{12} \mathrm{~S}_{2} \quad$ or $\quad \mathrm{C}_{100} \mathrm{H}_{48} \mathrm{~N}_{1} \mathrm{O}_{12} \mathrm{~S}_{2}$

Various researchers have investigated pyrolysis of carbonaceous chondrites and release of volatiles from this "polymer/kerogen" matrix material: Kerridge, 1985; Robert and Epstein 1982; Levy et al.,1973; Studier et al 1972. From the reports of these workers, stepwise heating of carbonaceous chondrite meteorites gives sequential volatiles release, as follows:

initially: $\mathrm{CO}, \mathrm{CO}_{2}, \mathrm{H}_{2} \mathrm{O}$; all at low temperature $(\sim 100 \mathrm{C})$ in "large" quantities; (unfortunately not measured)

then $\mathrm{C}_{2} \mathrm{H}_{4}, \mathrm{CH}_{4}, \mathrm{C}_{3} \mathrm{H}_{6}$ etc in "increasingly smaller quantities" at higher temperatures;

finally more $\mathrm{CO}$ released at $\mathrm{T} \cong 600^{\circ} \mathrm{C}$ (possibly due to autoreduction of $\mathrm{FeO}$ ?)

The implication of these findings is that asteroids having the composition of carbonaceous chondrites are legitimately able to be considered as orebodies for extraction of water and /or hydrocarbon volatiles.

Unfortunately, quantitative yields are not given, nor is there information to indicate required heating times for volatiles release. There is a need for simple experimental work on carbonaceous chondrites to determine lowest ultimate temperature for efficient volatiles extraction, and fastest heating rate for volatiles extraction, so as to be able to begin design of extraction processes. 


\subsubsection{Asteroid Taxonomy and Inferred Compositions}

Asteroid classification schemes have been based on identifying groupings of photometric and spectroscopic characteristics. Below are listed the various techniques:

Table 2.3 Asteroid Observation Methods

\begin{tabular}{|l|l|}
\hline Technique & Information derived and interpretations \\
\hline $\begin{array}{l}\text { Reflectance spectroscopy } \\
\text { and multicolor photometry }\end{array}$ & $\begin{array}{l}\text { Asteroid Classification; } \\
\text { inferred surface mineralogy [Requires broad spectral } \\
\text { coverage, high resolution, and high signal-to-noise } \\
\text { ratio: knowledge of albedo improves } \\
\text { characterization] } \\
\text { Detection of water-bearing materials }\end{array}$ \\
\hline $\begin{array}{l}\text { Visible photometry and } \\
\text { lightcurve photometry }\end{array}$ & $\begin{array}{l}\text { Size [Requires knowledge of albedo] } \\
\text { Albedo [Requires knowledge of size] } \\
\text { Rotation period [Requires a sequence of closely } \\
\text { spaced observations over several nights] } \\
\text { Approximate shape [From analysis of lightcurves] } \\
\text { Orientation of spin axis [From variation of lightcurve } \\
\text { form with viewing geometry] }\end{array}$ \\
\hline Visible polarization & $\begin{array}{l}\text { Albedo [Requires observations over a range of phase } \\
\text { angles] "there is an empirical reln. between albedo and } \\
\text { polarization of reflected light, and phase angle" }\end{array}$ \\
\hline Occultations & $\begin{array}{l}\text { Diameter [Dependent on obtaining accurate durations } \\
\text { from several sites]; existence of satellites }\end{array}$ \\
\hline $\begin{array}{l}\text { Infrared photometry } \\
\text { photometry] } \\
\text { metative emissivity [Model-dependent indication of }\end{array}$ \\
\hline
\end{tabular}




\section{Table 2.3 (continued)}

\begin{tabular}{|l|l|}
\hline Technique & Information and Interpretations \\
\hline Radar & $\begin{array}{l}\text { Surface conductivity or metal abundance [Model } \\
\text { depends on assumptions of surface porosity] } \\
\text { Diameter [From duration of returned signal] } \\
\text { Rotation rate [From frequency spread] } \\
\text { Shape [From temporal variation of frequency spread] }\end{array}$ \\
\hline $\begin{array}{l}\text { Passive microwave } \\
\text { spdiometry (incl IRAS) and }\end{array}$ & $\begin{array}{l}\text { Near-surface temperatures } \\
\text { Temperature gradients, conductivities, and thermal } \\
\text { inertias; cross-sectional area and albedo }\end{array}$ \\
\hline Space telescope images & $\begin{array}{l}\text { Moderate resolution images [Approximately 30-km } \\
\text { resolution in middle of asteroid belt] }\end{array}$ \\
\hline
\end{tabular}

From the accumulated meteoritics knowledge and from the classication efforts of Tholen \& Barucci (1989), and others, based on photometric and colorimetric properties, there has developed a tentative concensus regarding asteroid class-meteorite class match (eg Gaffey and McCord 1982, Lagerkvist \& Banucci, 1992).

Barucci's classification is based on 7 spectrophotometric colours and on IRAS-derived albedos, and shows that the population can be statistically split into 9 classes, denoted B, E, G, C, M, D, S, A. Subclasses are numbered $0,1,2$, etc with increasing value for higher albedos. This is described more fully in Tholen \& Barucci (1989). Inferred mineralogies are in Table 2.4 below.

\section{Asteroid Taxonomy and Inferred Compositions}

Despite the tentative identifications below, meteorites are an incomplete and misleading analogue of asteroidal material: they do not cover the D, P type asteroids at all, and they tell us effectively nothing about regolith mechanical properties. They do indicate mineral assemblages, but incompletely, and only of the surface material.

A major discovery of the last several years is that approximately two-thirds of Main Belt C-type asteroids show spectral evidence of water of hydration. (Jones, 1990, quoted in 
Davis et al., 1993). Rivkin \& Lebovsky 1995 found that "over 70 asteroids have been studied in the $3 \mu \mathrm{m}$ region for hydrated minerals, and these minerals have been found in roughly half of asteroids surveyed. Recent years have seen hydrated minerals discovered on asteroids of the $\mathrm{E}$ and $\mathrm{M}$ classes, previously thought to be anhydrous." In addition, the NEAs appear to exhibit the full diversity of compositions found thoughout the Main Belt, not being bound by the compositional gradation with solar distance that one finds within the Main Belt (Figure 2.4).

Table 2.4 Asteroid Taxonomy (modified from Nelson et al, 1993)

\begin{tabular}{|l|l|l|}
\hline Type & Interpreted mineralogy & Meteorite analog \\
\hline V & pyroxene, feldspar & howardite, eucrite, diogenite \\
A & olivine, olivine-metal & brachinite, pallasite \\
S & metal, olivine, pyroxene (1) & pallasite, mesosiderite \\
$\mathrm{K}^{*}$ & & carbonaceous / ordinary chondrites \\
M & metal, enstatite & irons, enstatite chondrites? \\
$\mathrm{R}^{*}$ & pyroxene, olivine & \\
$\mathrm{Q}^{*}$ & olivine, pyroxene, metal & ordinary chondrites \\
$\mathrm{E}$ & enstatite & enstatite achondrites \\
$\mathrm{T}^{*}, \mathrm{D}, \mathrm{P}^{*}$ & organic-rich silicates, carbon & \\
$\mathrm{B}, \mathrm{C}, \mathrm{F}^{*}, \mathrm{G}$ & hydrated silicates, carbon, organics, & CI, CM chondrites, black, gas-rich \\
$(2)$ & opaques, shock or radiation- & chondrites? \\
& darkened silicates & \\
\hline
\end{tabular}

(*) Asterisked letters do not appear in Barucci's scheme, but are used in some other classifications

(I) N.B. The density of Ida, an unambiguous S-type, as revealed by Dactyl's period, is such that it cannot contain much iron (unless its porosity is greater than 50\%), hence pallasite meteorites should no longer be considered derived from S-type asteroids.

(2) " $P, D, R D, T, F, G$, and $B$ are all C-type subclasses, and hence are probably rich in carbon and volatiles." - Nichols, 1993. 


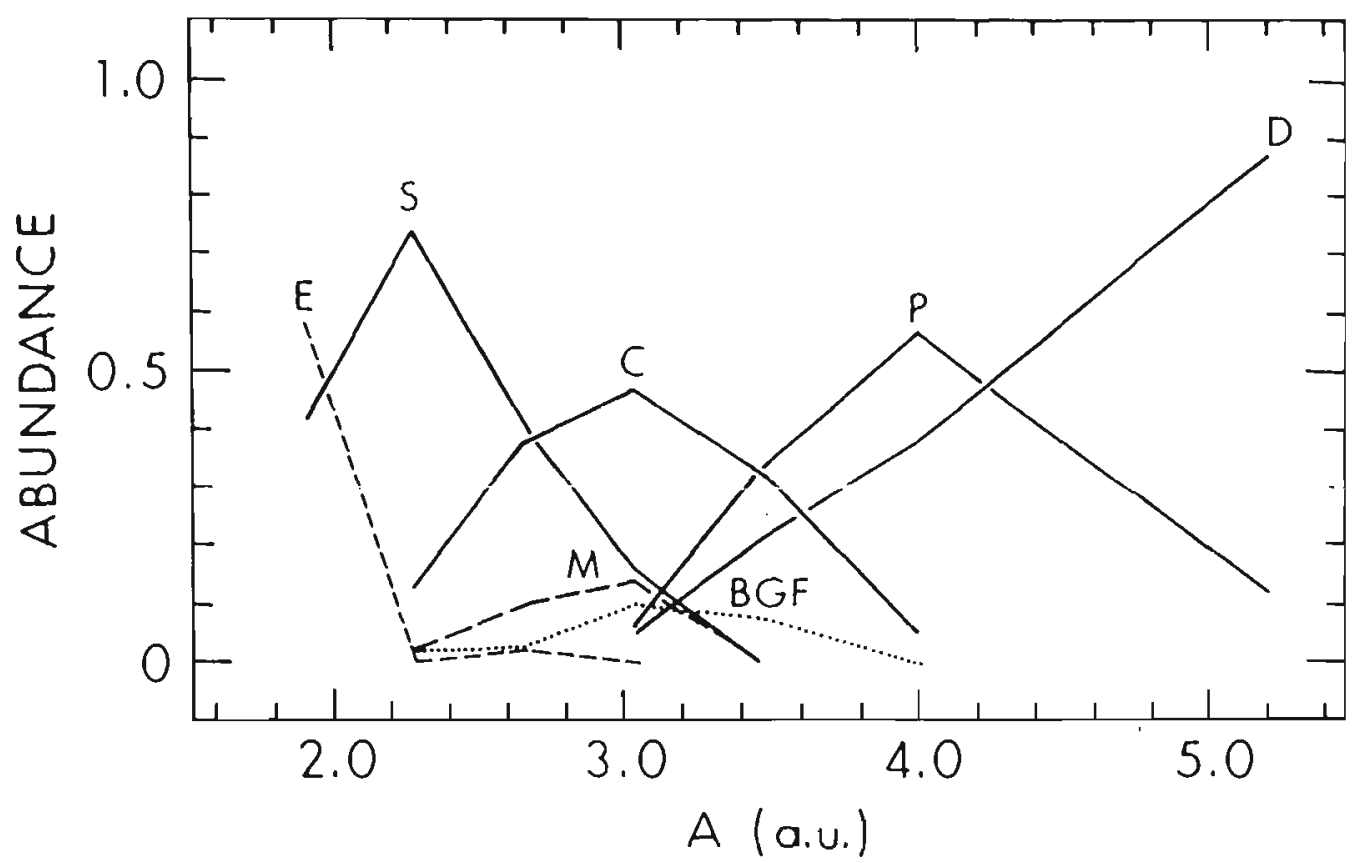

Plot of observed relative type distribution of the major taxonomic types as a function of semimajor axis. (Figure adapted from Bell et al. [1989]).

Figure 2.4 Compositional Gradation with Semimajor Axis (from Lewis, 1991)

Also, approximately half of the NEAs are believed to be derived from, or consist of, extinct comets. (Indeed, Steel suggests that a sizeable proportion are genetically related to $\mathrm{p} /$ Encke as apparent members of the Taurid Complex (Steel, 1995). Shoemaker suggests that comet - origin NEAs are up to $70 \%$ of the total. (pers.comm. JS Lewis). In fact, it appears to be now accepted that there will be objects which fit into an evolutionary progression from blatant comet to rocky asteroid devoid of gases. (See image of p/Halley, on page 24; this is the most detailed image of a comet yet obtained.)

Thus the majority of NEAs appear to contain at least some water, even if only present as water of hydration or chemically bound in clay minerals, and at least some readily extractable metal; they also appear to contain at least some $\mathrm{CO}_{2}$ source materials, either $\mathrm{C}$-polymer together with $\mathrm{FeO}$, or calcite/dolomite. 
Thus our targets have characteristics ranging from frozen mudballs or gravel-clad snowballs to strengthless gravel-banks, solid rocky mountains with or without detrital cover, to flying lumps of stainless steel, coal, or oil-shale!

Asteroidal regolith, where present (believed never to be present on bodies less than $2 \mathrm{~km}$ in diameter, until the discovery of Dactyl) is believed to be of very low strength, e.g., like dry sand or soil, very loose, high porosity, and very poorly sorted, ranging from micronsized particles to boulders.
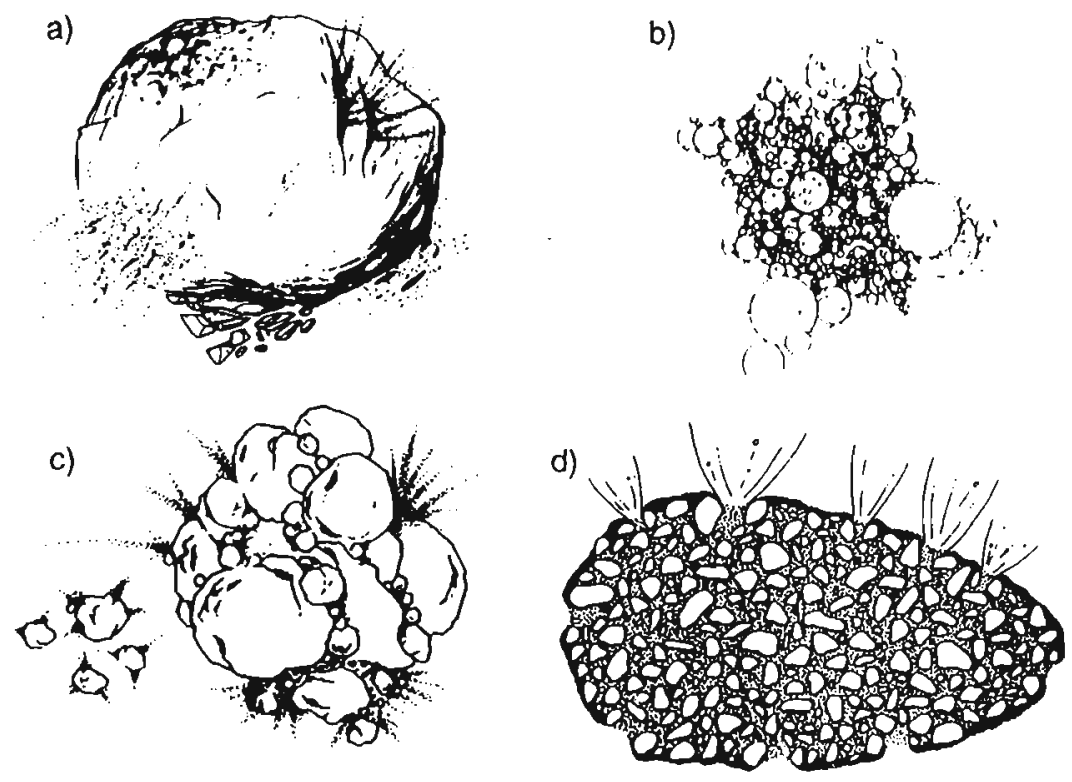

Four suggested models for the structure of cometary nuclei: (a) the icy conglomerate model (Whipple 1950; drawing from Weissman and Kieffer 1981); (b) the fractal model (Donn et al. 1985); (c) the primordial rubble pile (Weissman 1986); and (d) the icy-glue model (Gombosi and Houpis 1986). All but (d) were suggested prior to the Halley spacecraft encounters in 1986.

Figure 2.5 Comet Models (from Weissman 1994)

from top left: icy conglomerate (Whipple); fractal (Donn);

priordial rubble pile (Weissman); icy glue (Gombosi \& Houpis) 
This apparent resource richness and apparent surface 'tractability' tempts one to consider that which most professional metallurgists would seek to avoid, a plant designed to extract two or more products; but which the mining engineer loves: a multiproduct mine!

Further discussion is given in Chapter 6, Engineering Choices, Mining and Processing.

\subsubsection{Comet Origin of Some Asteroids}

There are strong indications that many asteroids are extinct or dormant comets (either because of the shape of their orbit, or because of telescopic/spectroscopic evidence), and thus potential sources of volatiles. Asteroid 4105 1979VA is known from its orbit to have been identified as comet $\mathrm{p} /$ Wilson-Harrington with a visible coma on its discovery apparition in 1949 (see e.g. Zuppero, Whitman, \& Sykes, 1993). Asteroid 1986TF is identified as comet p/Parker-Hartley 1989. Asteroid 2060 Chiron shows irregular outgassing and coma formation confirming a cometary nature. Lewis \& Hutson identify 1983SA (DonQuixote) as an "apparent extinct comet core".

Other probable and possible comet-origin asteroids are (Weismann and Campins, 1993):

Table 2.5 Suspected comet-origin asteroids

\begin{tabular}{|l|l|}
\hline probable comet origin & possible \\
\hline 944 Hidalgo & 1580 Betulia \\
2101 Adonis & 1620 Geographos \\
2201 Oljato & 1685 Toro \\
2212 Hephaistos & 1862 Apollo \\
3200 Phaeton (1983TB) in same orbit as Geminids & 1866 Sisyphus \\
3552 DonQuixote (1983SA) - a D-type Apollo & 1917 Cuyo \\
1984 KB & 1981 Midas \\
& 2062 Aten \\
\hline
\end{tabular}


Kuck has identified possible comet-origin asteroids from their Tisserand parameter; they include: 1986JK, 1987QB, 1994AB1, 1994JF1. The Tisserand parameter is defined in Chapter 3 and gives an indication of whether the orbit is under the influence of Jupiter (thus indicating likely membership of the family of "Jupiter comets").

In his use of the Tisserand parameter Kuck follows Hartmann, Tholen, and Cruickshank, 1987. Their listing is as follows:

strongest candidates:

$\begin{array}{ll} & \text { 1983SA } \\ & \text { 1984BC } \\ & \text { Chiron (now proven) } \\ \text { weaker candidates: } & \text { Griqua } \\ \text { Hilda } & \text { Thule } \\ \text { Chicago } \\ \text { Normannia } \\ \text { 1979VA (now proven) }\end{array}$

As noted by Duncan Steel, asteroids which are associated with meteor streams are more likely to be derived from, or to be remnants of, disintegrated comets, or may themselves be dormant or extinct domets (Steel, 1995).

\subsubsection{Comet Mantle Model}

Cometary material is probably of high porosity. Britt, Kring, \& Bell, 1995, suggest asteroidal material is probably also of high porosity, say $40 \%$ void spaces (e.g., like snow). This may arise as a result of very poor size sorting, lack of compaction forces, and also relates to the very skeletal structure of Interstellar Dust Particles.

Cometary material (and at least some asteroidal material) is probably of very low strength (say 100 to $1000 \mathrm{~N} / \mathrm{m}^{2}$ ) - a tiny fraction of the strength of ordinary rock. This is indicated by the evidence of comets which have broken apart, particularly during 
perihelion passage, and by the crater chains seen on various solar system bodies. Shoemaker -Levy - 9 was a recent spectacular instance of breakup under gravitational tidal forces. The details of the breakup of S-L-9 suggest that it was a zero-strength body, i.e., a "rubble-pile", of density much less than $1 \mathrm{gm} / \mathrm{cc}$..(Asphaug \& Benz, 1995).

Density is estimated to be approx $0.6 \mathrm{~g} / \mathrm{cc}$ by McKinnon \& Benner, 1995 from considerations of crater chains on Callisto and Ganymede.

Those asteroids which are "extinct comets" (1979VA Wilson-Harrington being apparently one) are believed to have various volatiles trapped at depth, such as water ice, frozen carbon dioxide, ammonia, and hydrogen cyanide ices, with silicates and hydrocarbons, under an insulating layer of remnant nonvolatile hydrocarbon and silicate detritus, called a "lag deposit" (Prialnik \& Mekler, 1991).

Prialnik \& Mekler's model proposed a porous dust mantle and a dense sub-mantle ice crust overlying a core of porous ice.

If the dust mantle is "too thin" then sublimation occurs rapidly and the surface ice layer evaporates faster than any inward-advection-produced ice crust can build up. If dust mantle is "too thick", then the mantle quenches vapour production, and you get an extinct or dormant comet. For $\rho=0.2$ and $0.5 \mathrm{~g} . / \mathrm{cc}$ and dust mantles $5 \mathrm{~mm}$ and $1 \mathrm{~mm}$ thick, an ice crust forms, and expands to depth of $\sim 1.3 \mathrm{~m}$ and $2.2 \mathrm{~m}$ respectively (after 10 orbits).

With the build up of crust, sublimation rate drops, decreasing by "many orders of magnitude". Dust impedes outflow of vapour and hence enhances inward flow - which densifies the deeper ice layer, but also warms it.

As ice sublimes and vapour streams away from comet surface, it entrains dust particles below a critical aerodynamic diameter, leaving coarser grains as a detrital layer. 
Their model suggests ice temperature (under mantle, at perihelion) of $100 \mathrm{~K}$ to $233 \mathrm{~K}$. (most likely $\sim 200 \mathrm{~K}$ ).

The implication of this model is that it is reasonable to believe that ices could survive for many orbits at depth under an insulating mantle.

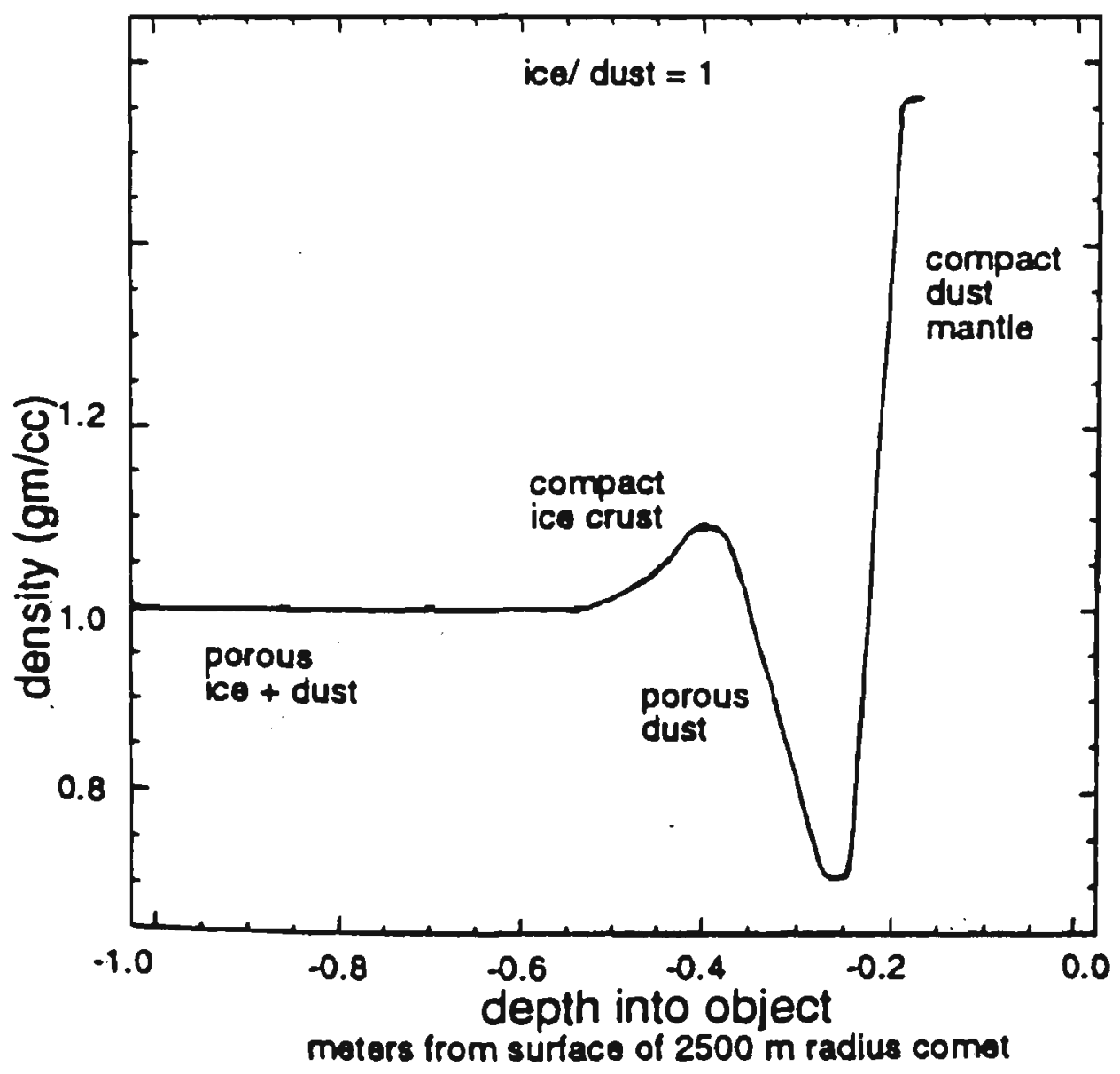

Figure 2.6 Density Profile of a Dormant Comet (from Zuppero et al, 1993) 


\subsection{Conclusions}

The new view of asteroids now emerging is that there appears to be a continuum in types from cometary through highly carbonaceous coal-like or oil-shale - like to hydrated silicates, anhydrous silicates, and metal. Unexpectedly, even the smallest of objects appear to be capable of developing a regolith. 


\section{Chapter 3 : Asteroid "Geography"}

\subsection{Introduction}

Most asteroids orbit the Sun in the Main Asteroid Belt, between 2 and $3.2 \mathrm{AU}$ (Astronomical Units) from the Sun (Earth-orbit radius is $1 \mathrm{AU}$ ). Also, most asteroids have their orbits inclined only slightly to the general orbital plane of the solar system. The objects of interest in this thesis are the Earth-approaching asteroids.

\subsection{The Earth-approaching or Near-Earth Asteroids.}

The Near-Earth asteroids (NEAs) are classified into the Apollos, Amors, and Atens, as follows:

$\begin{array}{ll}\text { Apollos: } & \mathrm{q}<1.017 \mathrm{AU} \\ & \text { Q unconstrained } \\ & \mathrm{a}>1.0 \mathrm{AU} \\ & \text { e, i unconstrained } \\ & \\ \text { Amors: } & \mathrm{q}>1.017 \mathrm{AU} \\ & \mathrm{Q} \text { unconstrained } \\ & \mathrm{a}>1.0 \mathrm{AU} \\ & \mathrm{e}, \mathrm{i} \text { unconstrained }\end{array}$

Atens: $\quad \begin{aligned} & \mathrm{q}<1.0 \mathrm{AU} \\ & \mathrm{Q}>1.0 \mathrm{AU} \\ & \mathrm{a}<1.0 \mathrm{AU} \\ & \\ & \mathrm{e}, \mathrm{i} \text { unconstrained }\end{aligned}$




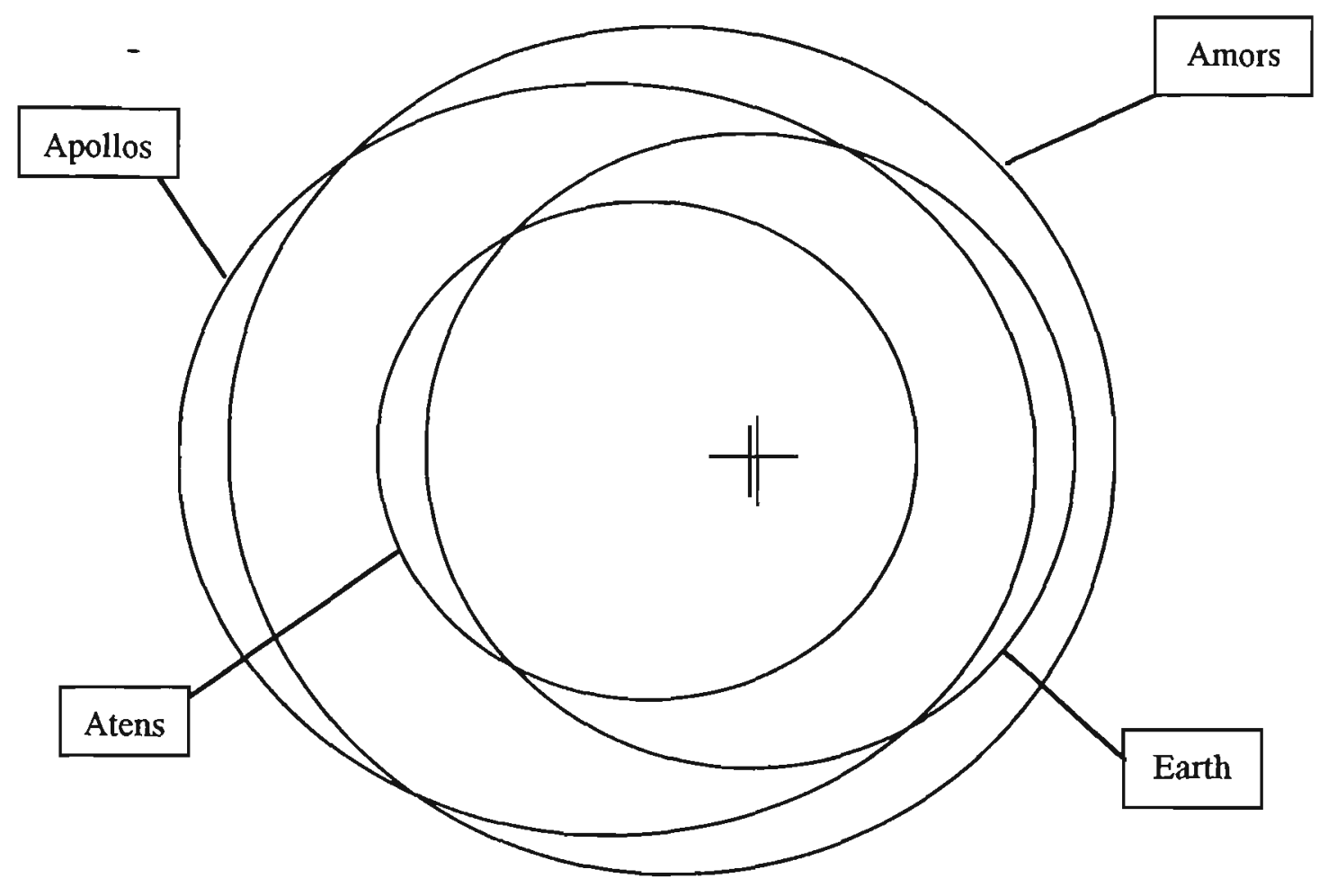

Figure 3.1 Apollos, Amors and Atens

The population of the NEAs is known to be continually depleted by collision with the inner planets, by gravitational boost out of the solar system following close encounter with Jupiter, and by impact with the Sun following gravitational perturbation by Jupiter (Farinella et. al., 1994).

There must be mechanisms for delivering new bodies into the NEA population, otherwise there would by now be none left over from the initial population emplaced at the time of origin of the solar system. The required mechanisms for maintaining the NEA population are not known but are believed to include capture into lower orbit and gradual decline in activity of short period comets, until they no longer show any cometary properties; and collision between large main belt asteroids injecting fragments into lower orbits either directly or via the Kirkwood gaps.

\subsection{Databases}

The Steward Observatory Asteroid Relational Database (SOARD) and the Planetary Data System Small Bodies Node (PDSSBN) are intended to provide up to date 
information on the orbital parameters of all known asteroids, and are available on Internet (see Appendix: Internet Addresses, p194). The total number of discovered Near Earth Asteroids is now about 380 and is increasing at about 50 per year. The table and graph below indicate the likely total population of the NEAs, as estimated by Shoemaker and updated by Lewis. (Lewis, 1993)

"Asteroid Listings", in the Appendices, at page 195, gives a recent listing of all low inclination Near Earth Asteroids. Also included is a listing of all NEAs with an orbital plane inclination less than 15 degrees, higher inclinations being essentially inaccessible with near-term propulsion systems.

Table 3.1 NEAR-EARTH ASTEROID POPULATION (from Lewis, 1993)

\begin{tabular}{|c|c|c|c|c|c|c|}
\hline \multirow{2}{*}{$\begin{array}{l}\text { Asteroid } \\
\text { Class }\end{array}$} & \multirow{2}{*}{$\begin{array}{l}\text { No. } \\
\text { Known }\end{array}$} & \multirow{2}{*}{$\begin{array}{l}\text { Projected } \\
\mathrm{D}>1 \mathrm{~km}\end{array}$} & \multirow{2}{*}{$\begin{array}{l}\text { Projected } \\
\mathrm{D}>0.1 \mathrm{~km}\end{array}$} & \multicolumn{3}{|c|}{$\begin{array}{l}\text { Number Easier to reach than Moon } \\
\text { (delta } V \text { out }<6.0 \mathrm{~km} / \mathrm{s} \text { from LEO) }\end{array}$} \\
\hline & & & & Known & $\begin{array}{l}\text { Projected } \\
D>1 \mathrm{~km}\end{array}$ & $\begin{array}{l}\text { Projected } \\
D>0.1 \mathrm{~km}\end{array}$ \\
\hline Aten $(\mathrm{a}<1.000)$ & 14 & 150 & 45,000 & 3 & 30 & 9,000 \\
\hline Apollo $(\mathrm{q}<1.017)$ & 113 & 1100 & 300,000 & 9 & 200 & 60,000 \\
\hline Amor $(q<1.300)$ & 89 & 750 & 220,000 & 11 & 150 & 45,000 \\
\hline TOTAL & 216 & 2000 & 565,000 & 23 & 380 & 114,000 \\
\hline
\end{tabular}

As seen from the table above, and from the graph overpage, the statistically predicted population of Near-Earth objects of diameter bigger than 100 metres, and even 500 metres, is very large indeed. 
Equivalent dlameters in kilometers

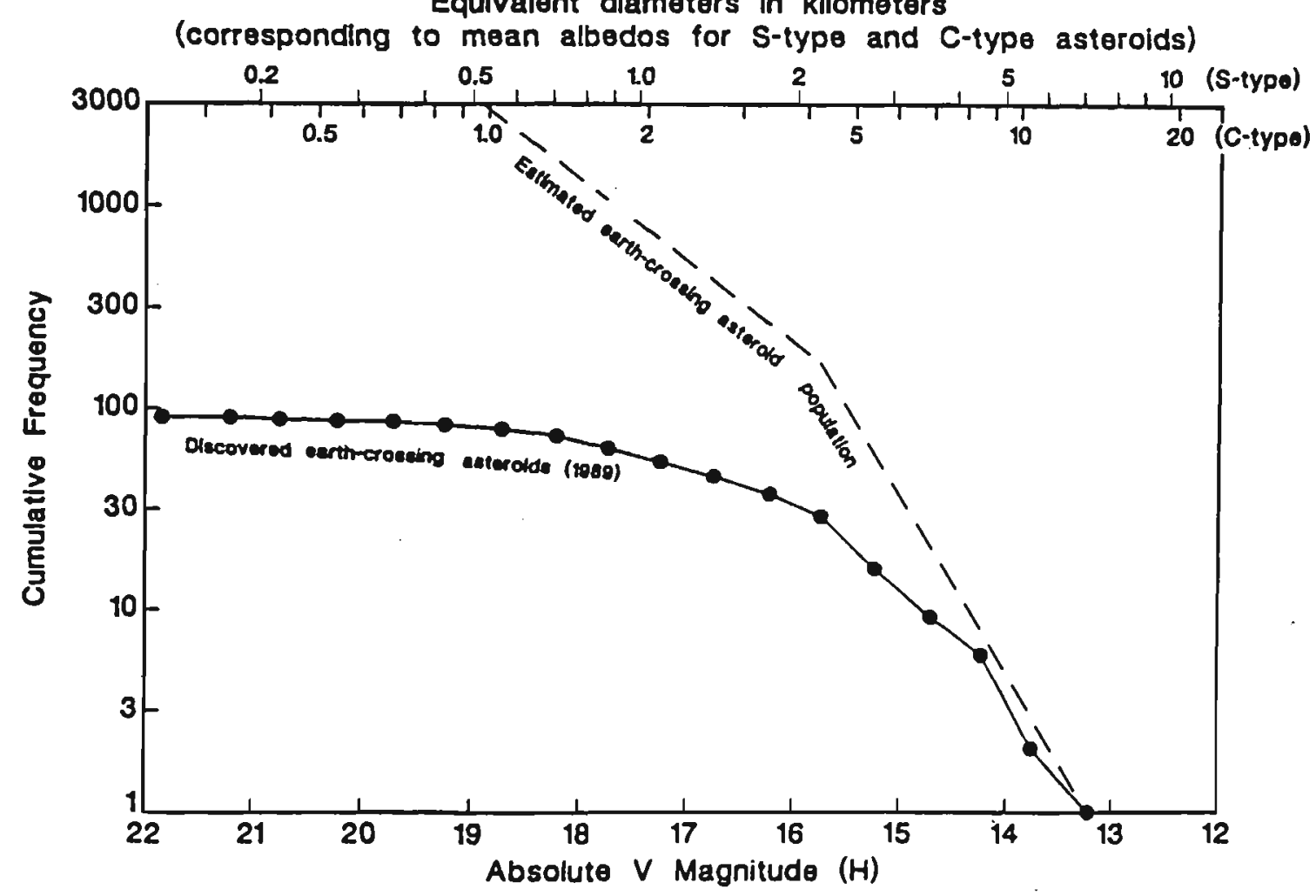

Figure 3.2 Size-Frequency Distribution (from Davis et al, 1993)

\subsection{Accessibility}

Discussion of target choice and of global minimum $\Delta \mathrm{v}$ calculations is given later.

However, asteroids 4660 Nereus (previously 1982DB) and 1989ML both have minimum $\Delta v$ 's less than $5 \mathrm{~km} / \mathrm{s}$, and as of 1991 there were 22 NEA's with minimum launch $\Delta v^{\prime} s$ 
under $6 \mathrm{~km} / \mathrm{s}$. Table 3.2 below gives accessibility of various NEAs compared with the Moon.

Table 3.2 Accessibility of Near-Earth Asteroids (from Lewis, 1991)

\begin{tabular}{|c|c|c|c|c|}
\hline Target & $\begin{array}{c}\text { Launch } \\
\text { Year }\end{array}$ & $\begin{array}{c}\text { Flight Time } \\
\text { (days) }\end{array}$ & $\begin{array}{c}\text { Outbound } \Delta V \\
(\mathrm{~km} / \mathrm{sec})\end{array}$ & $\begin{array}{c}\text { Inbound } \Delta V \\
(\mathrm{~km} / \mathrm{sec})\end{array}$ \\
\hline $1989 \mathrm{ML}$ & 2006 & 264 & 4.25 & $?$ \\
\hline $46601982 \mathrm{DB}$ & 2002 & 639 & 4.50 & 0.06 \\
\hline 1943 Anteros & 1997 & 731 & 5.29 & 0.39 \\
\hline $37571982 \mathrm{XB}$ & 1997 & 694 & 5.37 & 0.22 \\
\hline 3361 Orpheus & 1994 & 453 & 5.39 & 0.26 \\
\hline 1977VA & 2000 & 515 & 5.53 & \\
\hline 1980AA & 2001 & 703 & 5.54 & 0.36 \\
\hline $39081980 \mathrm{PA}$ & 2007 & 746 & 5.71 & \\
\hline Moon & any & 3 & 6.0 & 3.0 \\
\hline
\end{tabular}

Outbound $\Delta V$ is minimum total velocity change required to go from low-Earth orbit (LEO) to the surface of the body listed. Inbound $\Delta V$ is the $\Delta V$ required to go from the surface of the body to Earth intersection.

Mission velocities as calculated by the Shoemaker-Helin formula, given in Chapt 4, are presented in the Asteroid Listings Tables in the Appendices. Davis, Friedlander, and Jones (cited above) showed that the Shoemaker-Helin formula gives values within +/$20 \%$ of those calculated for actual missions. (see Fig 3.3.) The filled squares in Fig 3.3 are calculated total $\Delta v$ for actual specific launch opportunities. The dotted line is the Shoemaker-Helin calculation, which is intended to estimate a realistic 'global minimum'. As can be seen, Shoemaker-Helin is reasonably accurate, as a general formula, overestimating by about $0.5 \mathrm{~km} / \mathrm{s}$ for $1989 \mathrm{ML}$ and $1982 \mathrm{DB}$. 


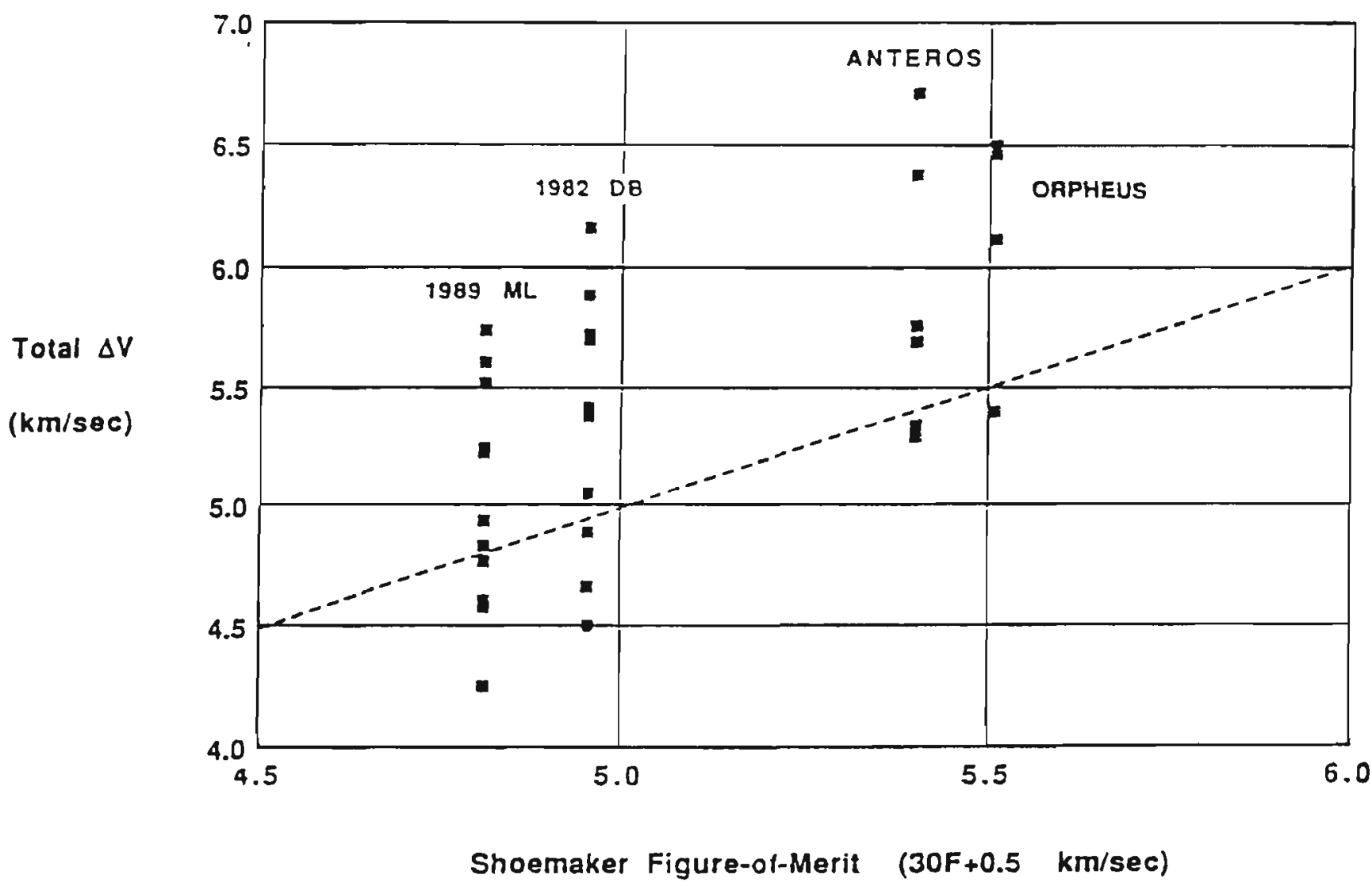

Figure 3.3 Shoemaker Figure of Merit c.f. specific missions (from Davis et al, 1993)

\subsection{The Arjunas}

Rabinowicz et al. have shown that there is an excess of small objects in nearly circular very"Earth-like" orbits, a near - Earth belt, which they have named the "Arjunas", less clearly defined as follows:

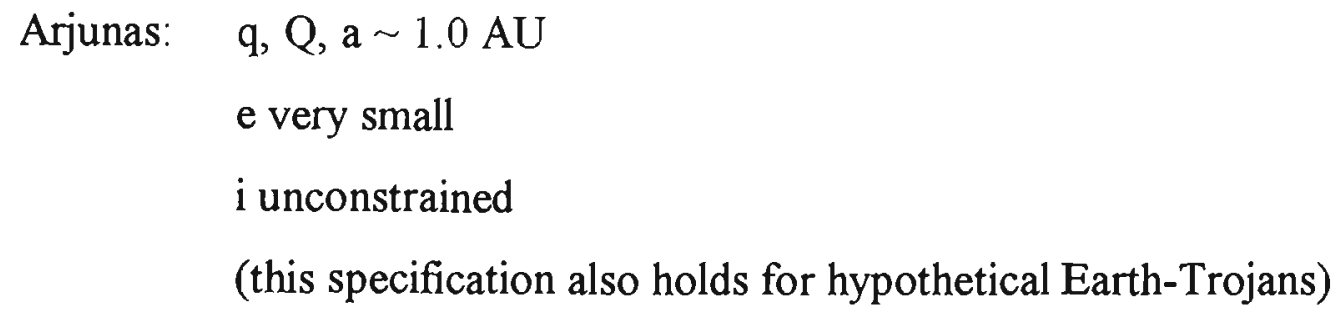

The hyperbolic $\Delta v$ for transfer to or return from these objects is likely to be under 1 $\mathrm{km} / \mathrm{sec}$. Dunbar has considered the possibility of Earth-Trojans and of bodies in Earthstabilized "horseshoe" orbits (Dunbar, 1979). The discovery of the Arjunas and the 
discovery of the Mars-Trojan, 1990MB Eureka makes it more likely that these hypothetical bodies may yet be discovered. These would be very difficult objects to discover with Earth-based telescopes, because of their large phase angle when closer than $1 \mathrm{AU}$, and dynamical prohibition of near approaches. They also would have very low energy requirements for transfer orbits to and from them. These bodies are close enough, energetically speaking, for material return missions to be considered without insitu propellant production.

A table of low-eccentricity NEAs (not all Arjunas) is given below:

Table 3.3 Minimum eccentricity Near Earth Asteroids

\begin{tabular}{|l|c|c|c|c|}
\hline Name & Eccentricity & Inclination & perihelion & aphelion \\
\hline 4581 Asclepius & 0.357 & 4.9 & 0.66 & 1.39 \\
1992BF & 0.271 & 7.25 & 0.66 & 1.15 \\
1991JY & 0.295 & 49.00 & 0.67 & 1.23 \\
1989UQ & 0.265 & 1.3 & 0.67 & 1.16 \\
1989UR & 0.356 & 10.34 & 0.70 & 1.47 \\
3554 Amun & 0.281 & 23.4 & 0.70 & 1.25 \\
2062 Aten & 0.182 & 18.9 & 0.79 & 1.14 \\
1982HR Orpheus & 0.322 & 2.68 & 0.82 & 1.60 \\
1991JW & 0.118 & 8.7 & 0.915 & 1.161 \\
1994UG & 0.246 & 4.5 & 0.925 & 1.527 \\
1991VG & 0.049 & 1.5 & 0.975 & 1.077 \\
1992JD & 0.032 & 13.5 & 1.002 & 1.067 \\
1993DA & 0.094 & 12.4 & 0.85 & 1.02 \\
\hline
\end{tabular}

(1991VG is the most accessible NEA presently known)

Of these objects, those with minimum inclination will be extremely accessible. The listing is far from complete, and as shown earlier, is being expanded on all the time. A one-year round trip to Arjuna 1991JW is shown in Figure 3.4. 


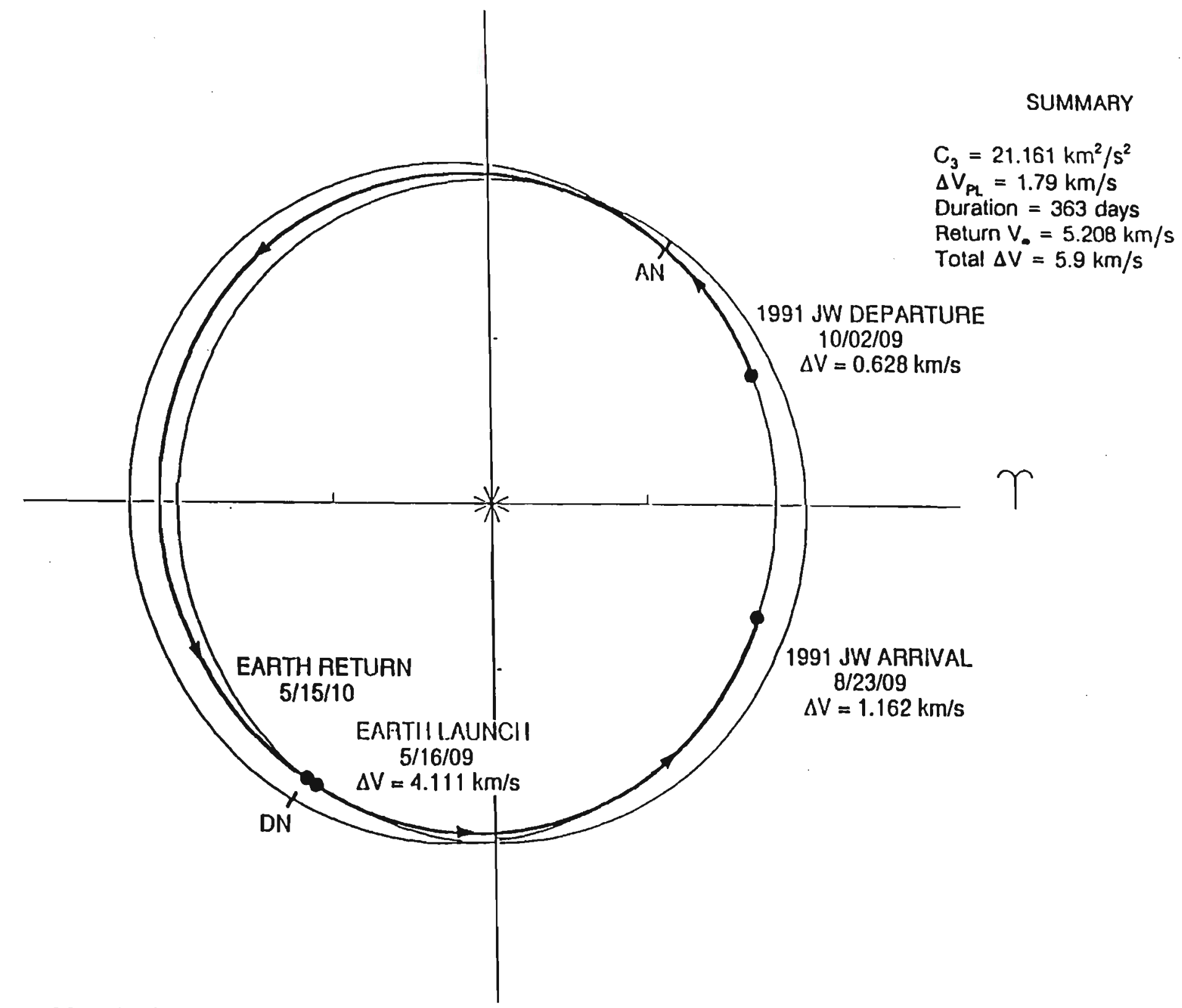

2009 LAUNCH OPPORTUNITY FOR A ONE-YEAR ROUND TAIP TO $1991 \mathrm{JW}$

Figure 3.4 Round Trip to $1991 \mathrm{JW}$ (from Davis et al)

(n.b. inclination to ecliptic is 8.7 degrees) 


\subsection{Short-period comets}

Given that there appears to be a genetic link between at least some of the NEAs and short period comets, it is appropriate to include some consideration of them as potential targets. Kuck has done this, and notes that very long trip times for ballistic aphelionrendezvous missions may be so disadvantageous financially that fast perihelion missions to short-period comets (with almost-guaranteed volatiles return) may be preferable, despite much higher delta-v requirement.

Table 3.4 Kuck's list of short-period comet targets

\begin{tabular}{|l|c|c|c|c|c|c|}
\hline Name & $\begin{array}{c}\text { Perihelion } \\
\text { (q) AU }\end{array}$ & $\begin{array}{c}\text { Aphelion } \\
\text { (Q) AU }\end{array}$ & $\begin{array}{c}\text { inclination } \\
\text { (degrees) }\end{array}$ & $\begin{array}{c}\text { Period } \\
\text { (years) }\end{array}$ & $\begin{array}{c}\text { eccent } \\
\text {-ricity }\end{array}$ & $\begin{array}{c}\text { Tisserand } \\
\text { parameter }\end{array}$ \\
\hline Boethin & 1.147 & 9.065 & 4.2 & & & \\
Churyumov- & 1.292 & 5.722 & 7.1 & 6.56 & & \\
Gerasimenko & 1.201 & 4.814 & 2.9 & 5.21 & 0.61 & 2.92 \\
du Toit-Hartley & 1.034 & 6.110 & & & 0.71 & 2.60 \\
Finley & 1.274 & 5.626 & 4.9 & 6.41 & & \\
Haneda-Campos & 1.034 & 5.871 & 13.5 & 6.41 & 0.70 & 2.78 \\
Hartley 2 & 0.528 & 5.514 & 4.3 & 5.25 & & \\
Honda-Myrkos- & 1.406 & 4.882 & 4.4 & 5.57 & & \\
Pajansakova & 1.584 & 5.351 & 4.7 & 6.46 & & \\
Howell & 1.367 & 6.202 & 4.2 & 7.36 & & \\
Kopf & 0.937 & 5.185 & 11.4 & 5.36 & 0.69 & 2.95 \\
Kushida & & & & & & \\
Schwassman- & 1.052 & 5.124 & 9.2 & 5.43 & 0.66 & 2.83 \\
Wachmann 3 & 1.159 & 4.524 & 4.5 & 4.79 & 0.59 & 3.02 \\
Tuttle-Giacobini- & 1.583 & 5.302 & 3.2 & 6.39 & & \\
Kresak & 1.059 & 5.132 & 11.7 & 5.44 & 0.66 & 2.64 \\
Wild 2 & 1.0003 & 4.287 & 2.8 & 4.3 & 0.62 & 3.08 \\
Wirtanen & & & & & & \\
Wilson- & 0.896 & 4.704 & 2.1 & 4.68 & 0.68 & 2.93 \\
Harrington & 1.135 & 4.468 & 3.5 & 4.69 & 0.59 & 3.04 \\
1986 JK & 1.159 & 3.763 & 3.5 & 4.05 & 0.48 & 3.27 \\
1987 QB & 1994 AB1 & & & & & \\
1994 JF1 & 1.317 & & & & & \\
\hline
\end{tabular}


Kuck used the Tisserand variable as an indicator of likely membership of the group of "Jupiter comets", in his review of asteroidal targets.

$\mathrm{T}=\frac{a_{j}}{a}+2\left(\frac{a}{a_{j}}\left(1-e^{2}\right)\right)^{0.5} \cos i \quad$ where $a_{j}=$ Jupiter's semi-major axis, and $\mathrm{T}<3$

indicates likely cometary origin.

As discussed in Chapter 9, only the objects with the smallest aphelia can really be considered to be prospective targets, because time duration will render an aphelion mission infeasible, and velocity requirement will render a perihelion mission infeasible.

\subsection{Conclusion}

The number of discovered NEAs is increasing rapidly. Many remain to be found. A substantial proportion are likely to be prospective "orebodies". The Arjunas in particular are very accessible. 


\section{Chapter 4: Orbital Mechanics}

This Chapter describes solar system orbit parameters and discusses various approaches for calculation of mission velocities for transfer between different heliocentric orbits.

\subsection{Orbital Geometry}

The position and orientation of an orbit with reference to the rest of the solar system is given by its semi-major axis, $a$; the inclination of the orbit's plane to the plane of the ecliptic (i.e., to the plane of Earth's orbit), $i$; the orbit's eccentricity, $e$; the longitude of the ascending node, $\Omega$; and the argument of the perihelion, $\omega$.

The longitude of the ascending node of an object's orbit is the angular distance, measured anticlockwise looking from the north, from the radius vector giving the Earth's position at vernal equinox (i.e., Earth's position on 21st Sept) to the position at which the object passes from below (south of) the earth's orbital plane to above (north of) the Earth's orbital plane. The argument of perihelion $\omega$ is the angular distance around the object's orbit from its ascending node to its perihelion, measured in the direction of rotation. (see figure 4.I)

\subsection{Delta-v (or mission velocity) as a measure of accessibility}

To depart from one orbit on a transfer trajectory to intersect another orbit requires application of a velocity change; and to rendezvous with the target body, i.e. to match velocity with it when the transfer trajectory intersects or is tangent to its orbit, also requires application of a velocity change. It is thus the total velocity change required to reach a body, rather than its distance, which is the true measure of the body's physical accessibility. 


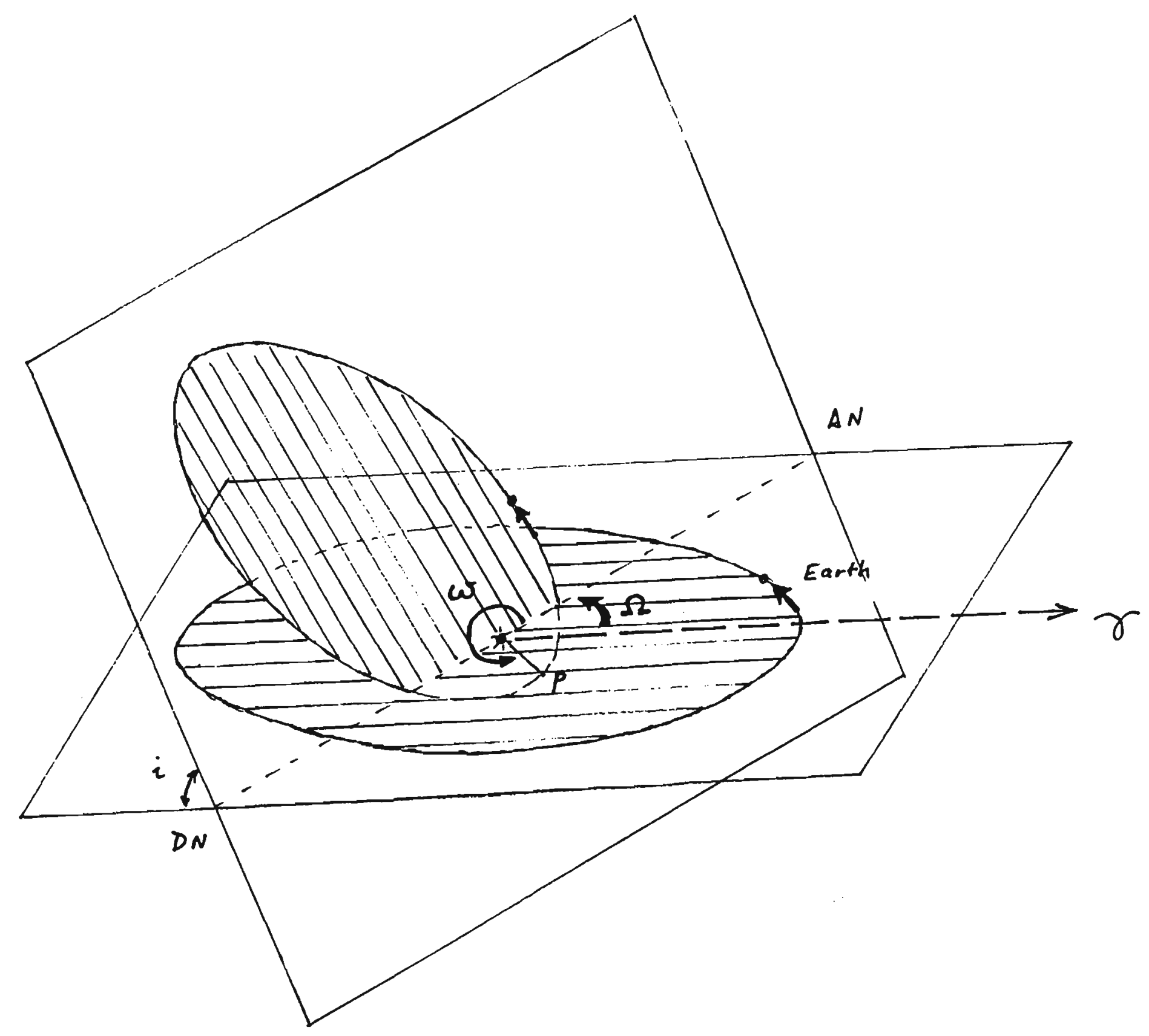

Figure 4.1 Geometry of Solar System orbits 


\subsection{Delta-v Calculations}

There are several approaches to estimating a general velocity requirement for any particular target, without zeroing in on a particular launch window. The most often referenced in the literature is the system of formulae presented by Shoemaker $\&$ Helin in 1978. An alternative is to use "first principles" calculations and this is described in Section 4.4.1. Time-specific calculations are more accurate, but are beyond the "proofof-concept" requirements of this overview.

Both eccentricity (and orbit semimajor axis) and target orbit inclination are critical as determinants of required mission velocity, as seen in Figure 4.2, from Davis et al..

\subsubsection{Calculation of Delta-v according to the formulae of Shoemaker \& Helin}

Shoemaker and Helin (1978) presented formulae for calculating $\Delta v$ to enter a transfer orbit to an NEA; they used an intermediary "Figure of Merit", F, where

$$
\Delta \mathrm{v}=(30 \mathrm{~F}+0.5) \mathrm{km} / \mathrm{s}
$$

Their formulae are as follows:

(1) Figure of Merit, $F=U_{L}+U_{R}$, where $U_{L}$ is the impulse required to inject the spacecraft into the transfer orbit from LEO; and $U_{R}$ is the impulse required to rendezvous with the asteroid The authors note that low $\Delta \mathrm{v}$ trajectories are achieved by rendezvous at or near aphelion or perihelion of the asteroid's orbit. Minimum $\Delta \mathrm{v}$ missions to Apollos and Amors are achieved by rendezvous at aphelion.

(2) With some qualifications, $U_{L}=\sqrt{U_{t}^{2}+s^{2}}-U_{o}$, where $\mathrm{s}$ is earth-escape velocity $(11.2 \mathrm{~km} / \mathrm{s})$, and $U_{o}$ is low-earth-orbital velocity $(8.0 \mathrm{~km} / \mathrm{s})$, and

Note that $U_{t}^{2}$ is more generally designated in space mission literature as $C_{3}$ the hyperbolic departure velocity squared (in $\mathrm{km}^{2} / \mathrm{s}^{2}$ ). 
Thus, $\mathrm{U}_{\mathrm{L}}$ is more normally given as

$U_{L}=\sqrt{C_{3}+(11.2)^{2}}-8.0 \mathrm{~km} / \mathrm{s}$

$U_{t}^{2}=3-\frac{2}{Q+1}-2 \sqrt{\frac{2 Q}{Q+1}} \times \cos \frac{i}{2}$

where $\mathrm{Q}$ is aphelion of the asteroid in $\mathrm{AU}$, and $i$ is inclination of its orbital plane to the ecliptic. They reference this equation to Opik, 1951.

$\mathrm{U}_{\mathrm{R}}$, the required impulse at rendezvous, is given as

$U_{R}=\sqrt{U_{c}^{2}-2 U_{r} U_{c} \cos \frac{i}{2}+U_{r}^{2}}$ where, for both Apollos and Amors,

$U_{c}^{2}=\frac{3}{Q}-\frac{2}{Q+1}-\frac{2}{Q} \sqrt{\frac{2}{Q+1}}$ and $U_{r}^{2}=\frac{3}{Q}-\frac{1}{a}-\frac{2}{Q} \sqrt{\frac{a}{Q}\left(1-e^{2}\right.} ;$ the present author has deleted, for simplicity, a plane-change term, $\cos \frac{i}{2}$, which multiplies with the surd in the $U_{c}{ }^{2}$ term for Amors, and with the surd in the $U_{r}{ }^{2}$ term for Apollos. This is reasonable for the cases of interest in this thesis, because for $i<10$ degrees, $\cos (i / 2)$ is still almost unity.

For Atens, minimum $\Delta \mathrm{v}$ missions are achieved by rendezvous at perihelion. Shoemaker and Helin do not however present formulae for perihelion rendezvous, but only for "short mission" aphelion rendezvous trajectories.

These are, $U_{t}^{2}=2-2 \sqrt{2 Q-Q^{2}} \cdot \cos \frac{i}{2}$; and $U_{c}{ }^{2}=\frac{3}{Q}-1-\frac{2}{Q} \sqrt{2-Q}$, with the other formulae above still applying. 
Shoemaker and Helin note that "a characteristic of special importance about rendezvous missions at aphelion with low $\Delta \mathrm{v}$ Amors and Apollos is that rendezvous impulse is very low, typically of the order of $1 \mathrm{~km} / \mathrm{s}$. Under optimum conditions, the departure $\Delta v$ for return to Earth is about the same."

Lau and Hulkower (1985) found that $10 \%$ of known NEAs had total $\Delta v \leq 6 \mathrm{~km} / \mathrm{s}$. They found that reasonable low $\Delta \mathrm{v}$ launch opportunities occur for NEAs with a period of about 2 years for each object.

They claim that global minimum total $\Delta \mathrm{v}$ is a "viable measure of accessibility". Unlike earlier more pessimistic assessments, they "demonstrated that asteroids (which) ranked high in the (accessibility) classification actually had more mission opportunities requiring less total $\Delta \mathrm{v}$ than those ranked low."

\subsubsection{Empirical formulae}

Cutler (1987) plotted $C_{3}, \Delta v_{D S}$, and total $\Delta v$ values for "ideal" opportunities given in Lau and Hulkower (referenced above), and produced "least squares" empirical formulae for predicting global minimum energy requirements. He found good correlation for $C_{3}$ and for total $\Delta v$ (i.e., departure $\Delta v$ plus deep space (rendezvous) $\Delta v$ ), with the following formulae:

$C_{3}=(34.615 \times a)-(9.0231 \times e)-(27.204 \times p)+(1.9280 \times i) \quad\left(r^{2}=0.951\right)$

total $\Delta v=(2.1161 \times a)-(1.6508 \times e)-(1.5273 \times p)+(0.19506 \times i) \quad\left(r^{2}=0.940\right)$

These least squares fits are surprisingly good, for an empirical, "sledgehammer" approach, and may therefore allow easy preliminary screening of candidate asteroids.

Cutler also plotted $\Delta v_{\text {out }}$ versus $\Delta v_{\text {return }}$ for various targets from Lau and Hulkower's specific mission data, and found "there is a general trend for.....many of the "best" mission opportunities to form a line of negative slope that defines an excluded region 
near the origin in which no mission opportunities lie." This behaviour implies that ideal minimum $\Delta v$ 's are not achieveable on both outbound and return trajectories for the same mission.

\subsection{Hohmann Transfer Orbits}

The lowest energy transfers are elliptical transfer between coplanar, semi-major axisaligned, elliptical orbits, tangent to the inner and outer orbits at perihelion and aphelion, respectively. The mathematics of these transfers is given in Section 4.4.1.

However, these "best case" situations are generally not available because the orbits

(i) do not have collinear semi-major axes and do not have their respective perihelion and aphelion 180 degrees apart; and/or

(ii) are not coplanar.

For the more general transfer situation, to/from an inclined elliptical orbit from/to a circular orbit, not in the same plane, the minimum energy transfer occurs when perihelion or aphelion is near ascending or descending node.

The $\Delta \mathrm{v}$ for plane change is additive to that for aphelion and perihelion change. The addition is vector addition, if both energy change and plane change occur simultaneously; but is arithmetic addition, if the two impulses have to occur at separate times.

At its simplest, $\Delta \mathrm{v}$ for inclination change is $0.5 \mathrm{~km} / \mathrm{s}$ times $\mathrm{i}$ (degrees), when change is made at $1 \mathrm{AU}$, at Earth's circular velocity. It is "cheaper", however, if the velocity change can be made at the asteroid's aphelion.

Figure 4.2 shows the combined effects of eccentricity and semimajor axis on minimum mission velocity. It implies that one should search for objects that meet the following criteria:

$0.05>\mathrm{e}>0.15 \mathrm{AU}$; and $0.95>\mathrm{a}>1.1 \mathrm{AU}$. 

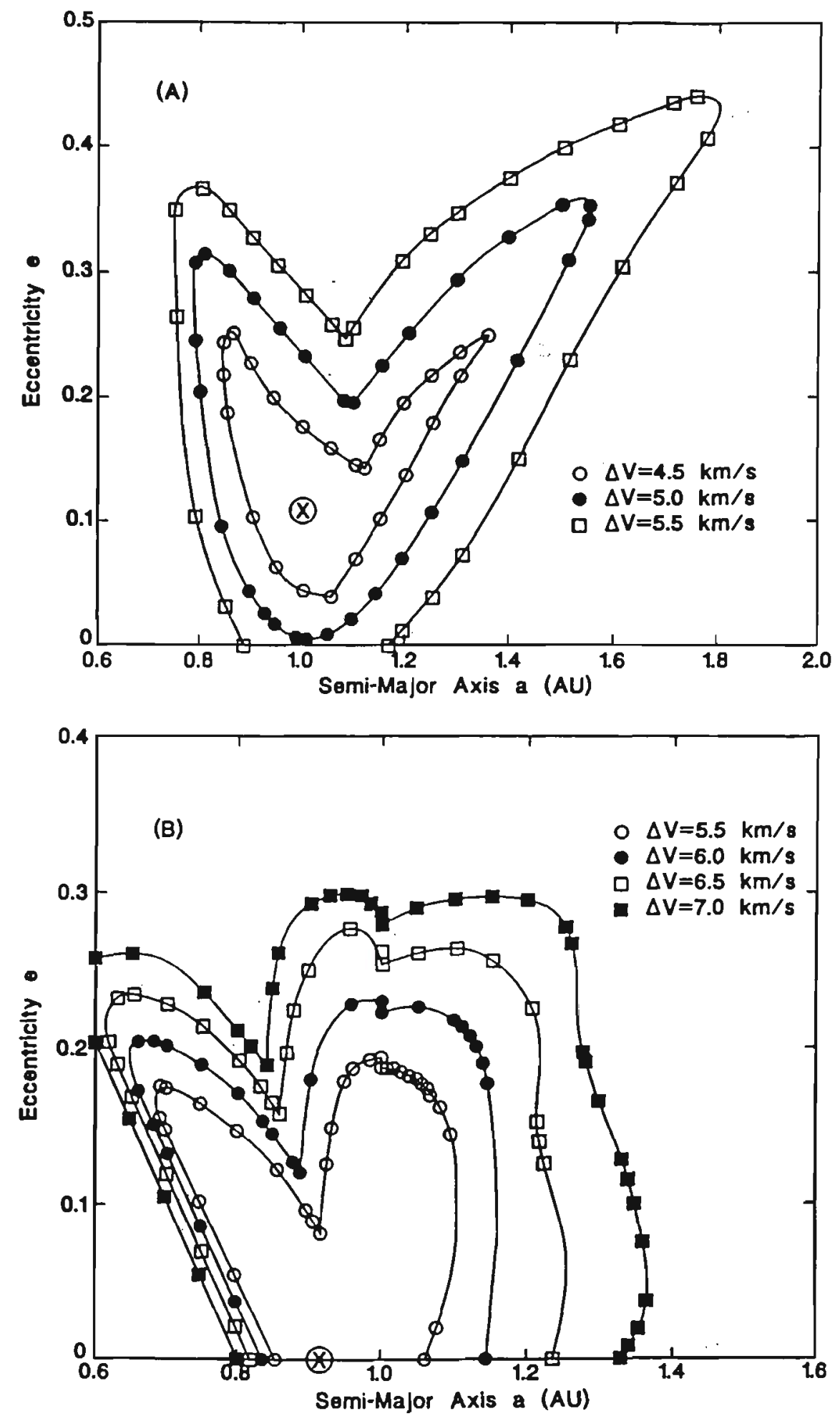

$\Delta V$ contours in $a-e$ space for $i=5 \mathrm{deg}$. Part (A) is for minimum-energy rendezvous, while part $(B)$ is for fast trip rendezvous.

Figure 4.2 Mission Velocity contours on Eccentricity - Semi-major Axis plot from Davis et al) 


\section{Hohmann Transfer Calculations}

The "first principles" calculation of velocity increments for carrying out a transfer from one elliptical orbit to another, coaxial, coplanar elliptical orbit is given below.

The minimum energy transfer from the lower (inner) orbit to the outer commences with a $\Delta \mathrm{v}$ (increase in velocity) at the periapse of the inner orbit, $\Delta \mathrm{v}_{\mathrm{p}, 1}$. This velocity increase must be of enough magnitude to raise the apoapse of the transfer orbit to be equal to the apoapse of the outer orbit.

At the point where the transfer orbit contacts the outer orbit, a second $\Delta v$ impulse is required (again in the forward direction) to increase velocity to match that of the higher orbit, i.e., raise the periapse to that of orbit 2 .

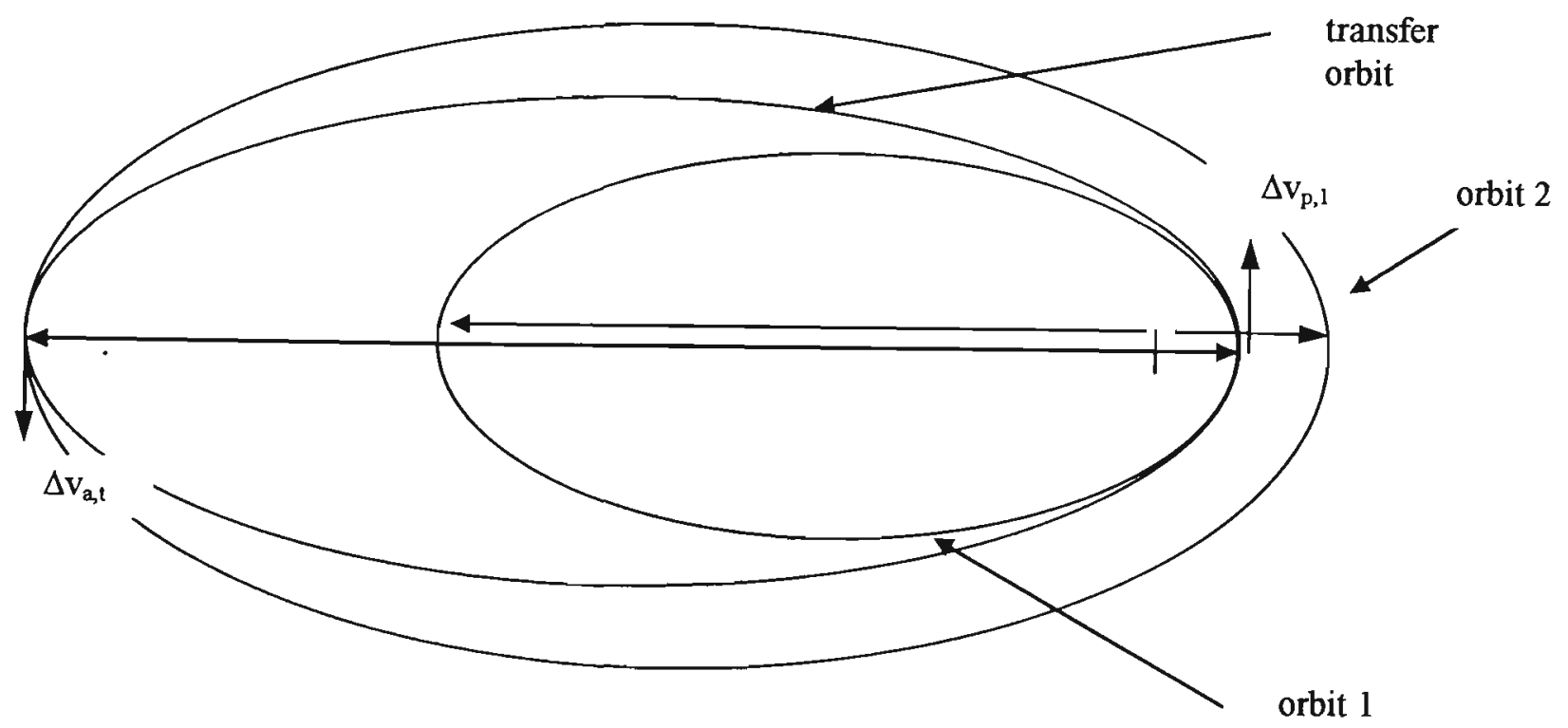

Figure 4.3 Hohmann Transfer Orbit

\section{Delta-v's for Elliptical Orbit Transfers}

The formulae below are taken from Thomson (1986) pp70-71:

The velocity at periapse of orbit 1 is: 


$$
v_{p, 1}=\sqrt{\frac{\mu\left(1+e_{1}\right)}{r_{p, 1}}} \quad \text { where } \mu=\mathrm{G} . \mathrm{m}_{\mathrm{s}}
$$

The transfer orbit must satisfy:

$$
v_{p, t}=\sqrt{\frac{\mu}{r_{p, 1}\left[\frac{2\left(r_{a, 2} / r_{p, 1}\right)}{1+\left(r_{a, 2} / r_{p, 1}\right)}\right]}}
$$

First (periapse) impulse is: $\Delta v_{p, t}=v_{p, t}-v_{p, 1}$

At apoapsis, the transfer ellipse velocity is found from the Conservation of Momentum:

$$
v_{a, t}=r_{p, 1} \cdot v_{p, t} / r_{a, 2}=\sqrt{\frac{\mu}{r_{a, 2}}\left[\frac{2}{1+\left(r_{a, 2} / r_{p, 1}\right)}\right]}
$$

- the required velocity at this point, to enter orbit 2 , is $v_{a, 2}$ :

$$
v_{a, 2}=\sqrt{\frac{\mu\left(1-e_{2}\right)}{r_{a, 2}}}
$$

Thus, second impulse is $\Delta v_{a, t}=v_{a, 2}-v_{a, t}$;

The total $\Delta \mathrm{v}$ for the Hohmann transfer is:

$$
\begin{aligned}
& \Delta v_{\text {total }}=\Delta v_{p, t}+\Delta v_{a, t} \\
& \Delta v_{p, t}=\sqrt{\frac{\mu}{r_{p, 1}\left[\frac{2\left(r_{a, 2} / r_{p, 1}\right)}{1+\left(r_{a, 2} / r_{p, 1}\right)}\right]}-\sqrt{\frac{\mu\left(1-e_{1}\right)}{r_{p, 1}}}} \\
& \Delta v_{a, t}=\sqrt{\frac{\mu}{r_{a, 2}}\left(1-e_{2}\right)}-\sqrt{\frac{\mu}{r_{a, 2}}\left[\frac{2}{1+\left(r_{a, 2} / r_{p, 1}\right)}\right]}
\end{aligned}
$$

Note also, $e=\frac{Q-q}{Q+q}$ and $Q=\frac{q(1+e)}{(1-e)}$ 
The $\Delta v$ for orbital plane inclination change is, at its simplest, when $i$ is small and the inclination change is made at $1 \mathrm{AU}$, at Earth circular velocity:

$$
\Delta v \quad=\quad 0.5 \mathrm{~km} / \mathrm{s} \times i \text { (degrees) }
$$

There is a relationship between the hyperbolic departure velocity of a departing spacecraft (its velocity relative to the Earth at a point outside Earth's gravity well: $v_{\infty}$ ) and the required velocity of departure from LEO ( $\mathrm{V}$ burnout, LEO ). It is:

$\left(\mathrm{v}_{\text {burnout }} \text { LEO }\right)^{2}=\mathrm{v}_{\infty}{ }^{2}+\left(\mathrm{v}_{\text {esc, }} \text { LEO }\right)^{2}$ or in words,

$(\text { LEO departure velocity })^{2}=(\text { escape velocity at LEO })^{2}+(\text { velocity at } \infty)^{2}$ eqn (11)

This comes directly from the Conservation of Energy.

The above formulae are used in calculations of $\Delta v$ requirements for the examples in Chapter 9. 


\section{Chapter 5: Mission Plans and Trajectories}

This Chapter considers the alternative out-and-return trajectories to different target bodies, taking into account allowable stay times for resource extraction. Five "mission types" are identified.

\subsection{Hohmann missions and timing considerations}

From consideration of the orbital locations of targets discussed in Chapter 3, we can see that there will be a variety of mission and trajectory types. This is because:

- $\quad$ targets may be in 'low' or 'high' eccentricity orbits;

- $\quad$ targets may have perihelion inside or outside earth orbit;

- $\quad$ transfer from target may be by Hohmann ellipse or by 'continuous thrusting';

- $\quad$ mining season may be 'short-term' or extended;

- $\quad$ mining season may be 'single-mission' or 'repeating';

- $\quad$ if 'short-term' mining season, it may be aphelion-centred or perihelion-centred.

For low-e targets: can be continuous thrusting return;

can be 'long' mining season ( $>90$ degrees of orbital arc);

can be repeating.

For high-e targets: must be impulsive transfer ( $<20$ degrees of orbital arc); must be short mining season; must be aphelion or perihelion mining season; must be "one-off".

\section{$\underline{\text { Timing Scenarios }}$}

The synodic period of a body, with respect to the Earth, is the time that elapses between similar configurations (e.g., conjunctions; oppositions).

$(\text { synodic period })^{-1}=+/-(\text { period of body })^{-1}-/+(\text { period of Earth })^{-1}$ 
Thus an Arjuna with a period of 15 months would have a synodic period of 60 months ( 5 years).

Orbit-matching and synodic period constraints militate against a general "pro-forma" approach to trajectory design. For example, it is necessary for the payload on its return trajectory to intersect Earth orbit when Earth is nearby (and not on the other side of the sun).

This implies longer project timelines than indicated simply by use of transfer orbit period, $\mathrm{T}$, because of the necessity for phasing orbits. In turn, these longer timelines impact negatively on Net Present Value.

On the other hand, they allow for a longer mining season and hence allow less demanding specifications on mining equipment and on solar collector/furnace.

However, if we contemplate a thrusting time/thrusting arc which extends through more than approx $15^{\circ}$ of anomaly or (say) 50 to 100 days immediately post- aphelion for an Apollo, Amor, or Comet, then the departure from impulsive, Hohmann, ballistic conditions is large, and the $\Delta v$ requirements increase, generally by up to 1.5 times.

In the worst case, with continuous-thrusting spiral trajectories, calculations have shown $\Delta \mathrm{v}$ to be twice that for Hohmann transfers.

Like terrestrial mining projects, we find that each asteroidal resource project will have its own idiosyncrasies, reflected here in the alternative mission trajectory profiles to be considered.

\subsection{Alternative Mission Types}

From a review of the orbital geometries we can see that there are various scenarios available: 


\subsection{1 “Apollo-Type": Apollo or high-e Amor asteroids}

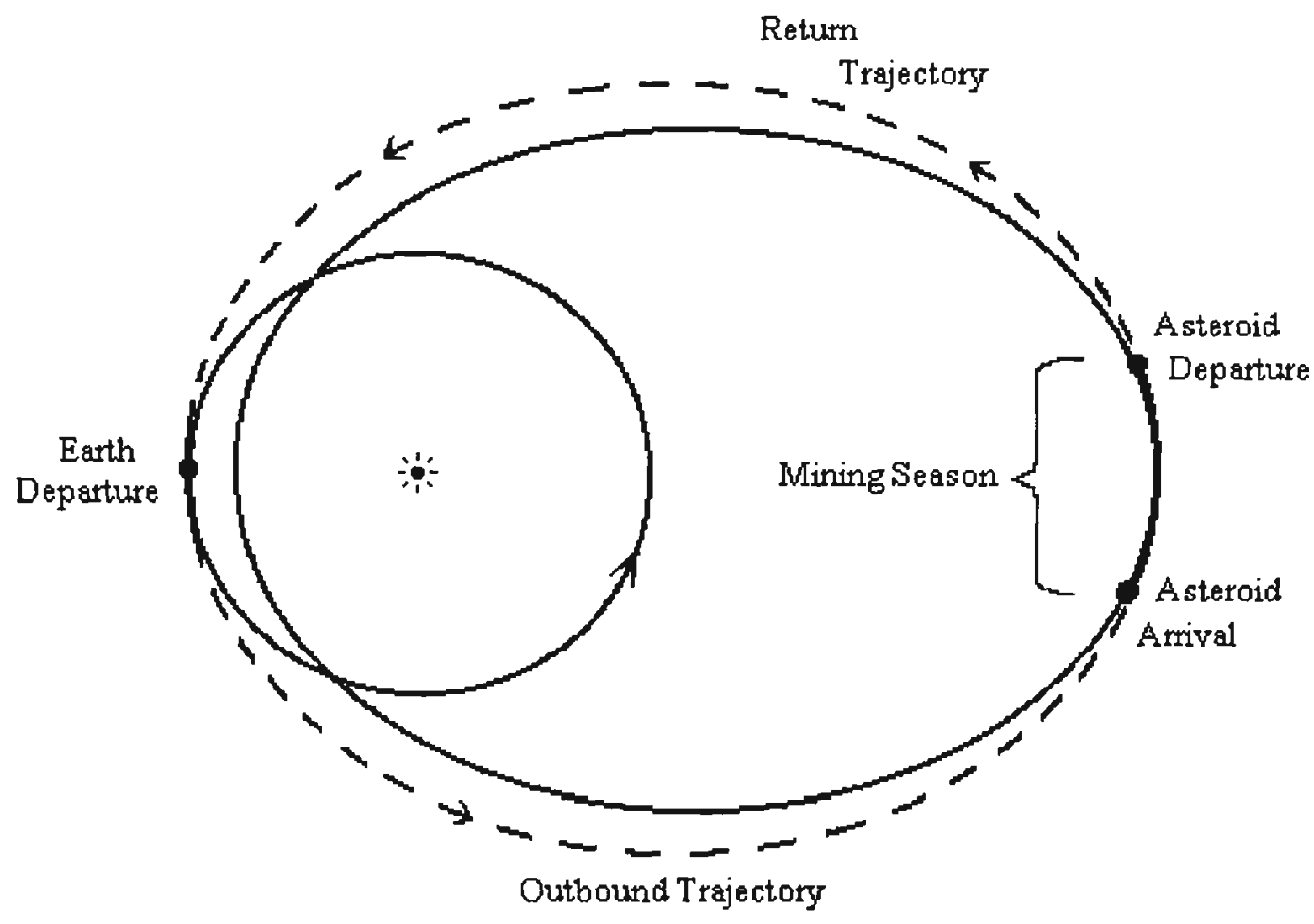

Figure 5.1 "Apollo-Type" Mission

Objects with "high" eccentricity, low-i orbits demand Hohmann transfer for both outbound and inbound trajectories, because of their relatively high delta- $\mathrm{v}$ requirement. Mining season is restricted to a short period during aphelion; $\Delta \mathrm{v}$ for return must be achieved in a small fraction of $\mathrm{T}$.

This trajectory applies to a target body which is in an elliptical orbit, a $>1$ AU, i.e., an Apollo, Amor or short-period comet; and assumes Hohmann transfer with rendezvous near but before aphelion for minimum $\Delta \mathrm{v}_{\text {out }}$; a "short" aphelion-centred mining season, (approx 3 month mining stay); and a post-aphelion departure for Earth-return, with approx 3 month thrusting, for minimum $\Delta \mathrm{v}$ return .

Such a mission encounters the problem of long project duration, which is undesireable as regards NPV. In addition, it does not allow good use of the time: only a small arc centred on aphelion is available for mining, and only a limited arc is available post- 
aphelion for boost into return trajectory. There is also a need to destroy a relatively large return (hyperbolic) arrival delta v. Lunar flyby is of partial use only, because it can remove only $1.5 \mathrm{~km} / \mathrm{s}$. This criterion, i.e., the delta- $v$ requirement to achieve Earthcapture, is in fact more demanding than the asteroid-departure delta-v requirement.

The use of Hohmann ellipse transfer out and back, and "short" aphelion-centred mining season, implies that mission duration must approximate the period $\mathrm{T}$ of transfer orbit which itself must approximate an integer no. of years (because the Earth has to be there when the payload gets back!).

Note therefore with regard to synodic period that either one adopts a low-thrust spiral trajectory approach or adopts a selection rule: that earth-body transfer orbits must be of period $=$ integer no. of years $( \pm 10-15 \%)$.

To minimise delta-v (deep space), the object's orbit should be "Earth-grazing", i.e., $q=$ $1.0 \mathrm{AU}$.

So, $\mathrm{T}_{\text {transfer orbit }}=2$ years implies a semi-major axis $=1.587 \mathrm{AU}$, and thus, with $\mathrm{q}=1.0$ AU, Q must be 2.174 AU. This gives aphelion at the inner edge of the Main Belt.

For $\mathrm{T}_{\text {transfer orbit }}=3 \mathrm{yrs}, \mathrm{a}=2.08 \mathrm{AU}$, and for $\mathrm{q}=1.0 \mathrm{AU}, \mathrm{Q}=3.16 \mathrm{AU}$.

This aphelion is at the outer edge of the Main Belt. 


\subsubsection{Short period comet missions:}

Perihelion rendezvous may be appropriate for mining short-period comets, as discussed by Kuck (1995), because (i) solar insolation is too weak at aphelion; (ii) more importantly, aphelion rendezvous imposes financially disastrous time delays (see orbital periods for short period comets in Kuck's list).

Dormant comets may be desireable targets because (i) drilling is assumed to achieve close to $100 \%$ recovery and capture of liberated volatiles; (ii) equipment for in-situ melting is likely to be considerably less massive than equipment for mining and processing regolith (possibly by factor of 10 ).

This is counter-balanced by the very much higher $\Delta v$ requirement for return, which translates into a requirement for much higher propellant usage on return transfer, and hence a larger "mining" requirement. Note also the imposition of very short mining season.

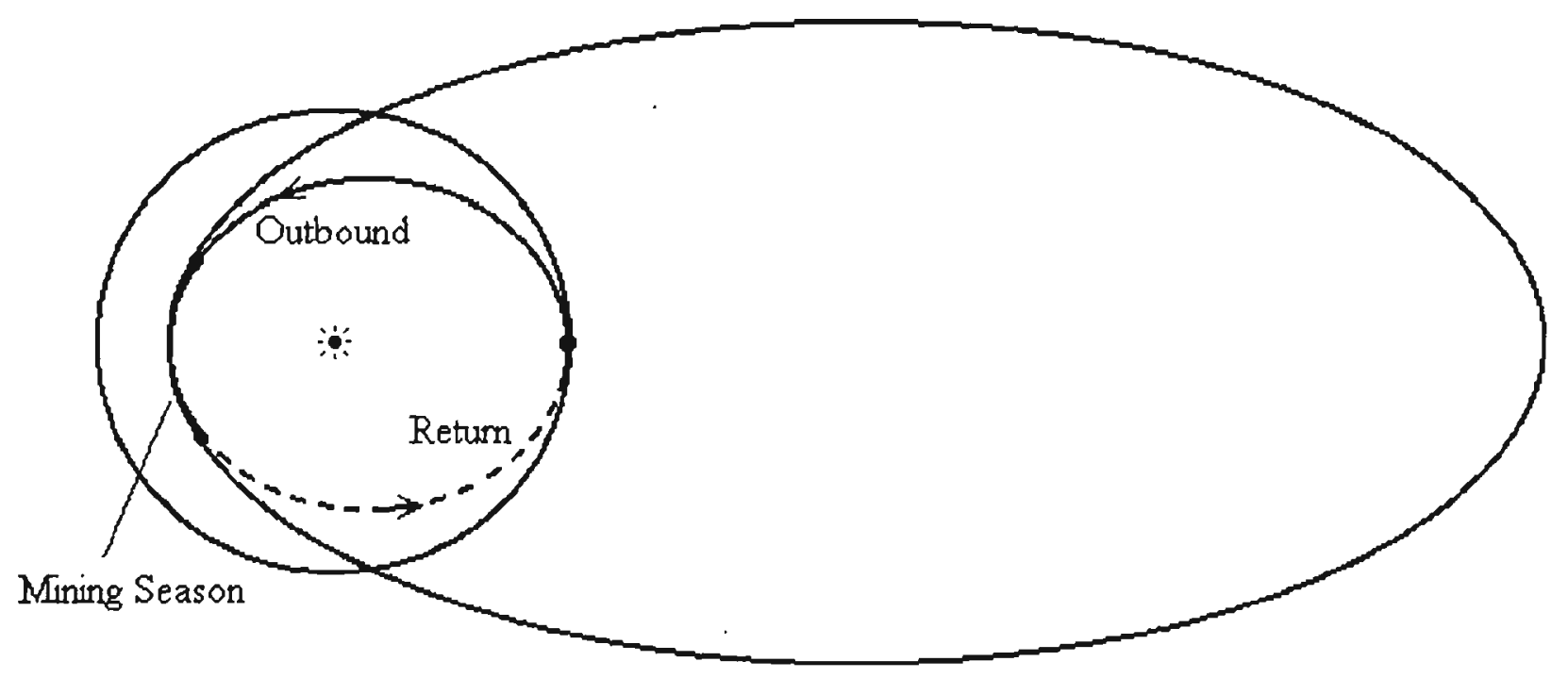

Figure 5.2 "Comet-Type" Mission 


\subsection{3 “Aten-Type": High-e Atens:}

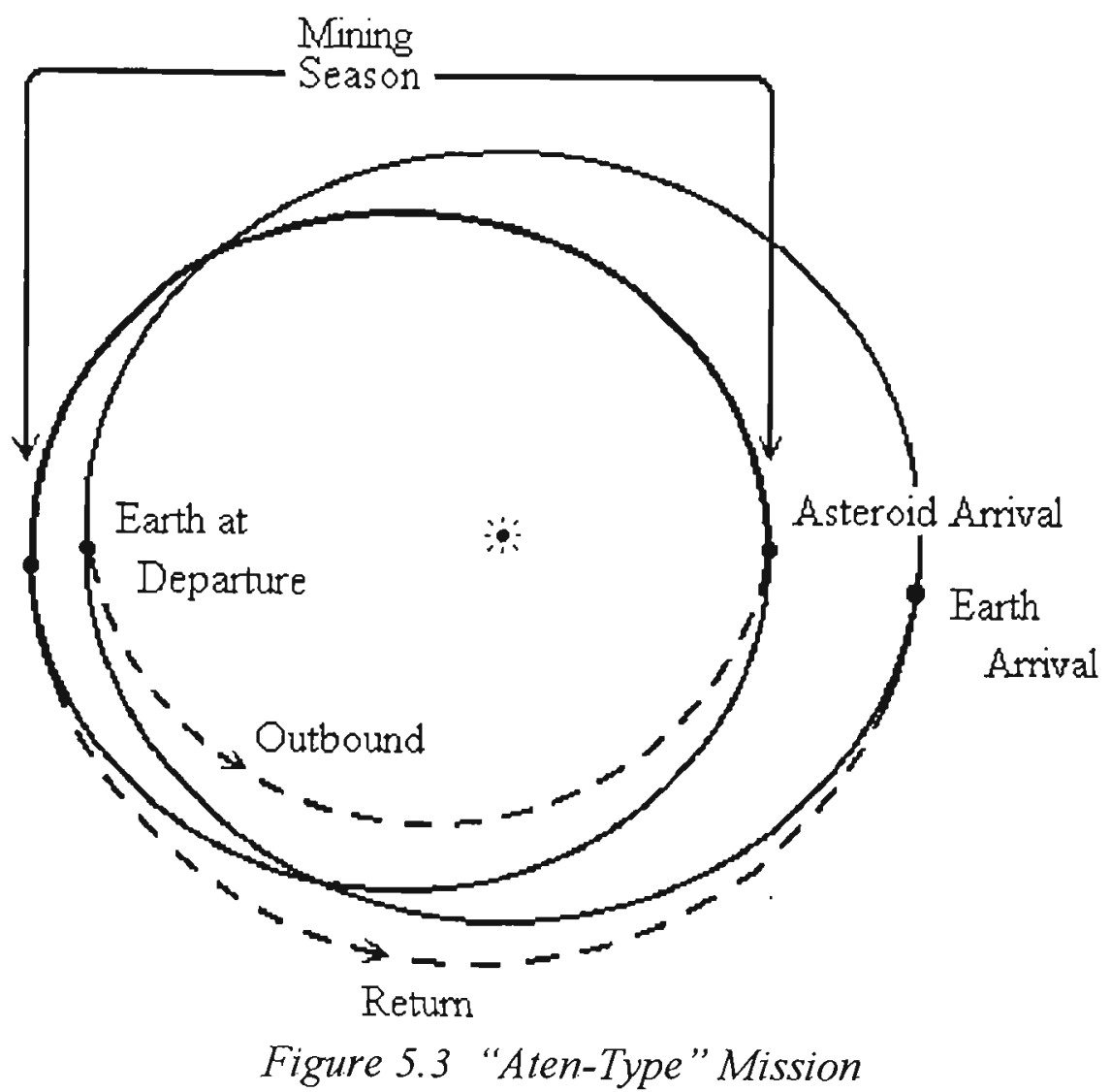

This mission type assumes a Hohmann transfer to rendezvous with the target asteroid at its perihelion; and near-aphelion departure after T/2 stay time. Post-perihelion departure is ruled out, because (i) this gives inadequate mining season duration; (ii) there is a phasing requirement : T of transfer orbit $<1 \mathrm{yr}$, so Earth will not 'be there' if return craft sets out from target's perihelion).

This mission profile assumes a target body a $<1$; elliptical orbit (an Aten); and a mining season commencing at perihelion, and running until aphelion. Note that phasing to achieve Earth rendezvous on return forces off-optimum transfer orbits anyway, thus one might contemplate an aphelion arrival (requiring high $\Delta v$ ds to rendezvous) and a perihelion departure for low return $\Delta v$ requirement.

This implies another "selection rule": [T/2 of transfer orbit to target's perihelion + T/2 of target's orbit + T/2 of transfer orbit from target's aphelion to Earth] is the mission time, and this must approximate to 1.5 years. 
Whether to choose perihelion or aphelion rendezvous for these "Aten-type" missions needs to be determined on individual basis, by checking $\Delta \mathrm{v}_{\text {out }}$ and $\Delta \mathrm{v}_{\text {retum }}$, and total time of mission. (see Chapter 9)

Perihelion mining season gives short project duration, which is favourable for NPV, but is very demanding propulsion system requirement, and is in any case, not amenable to phasing requirements.

If one were to abandon Hohmann missions, either for "Apollo-type" or for "Aten-type" missions, on the basis that the time constraints on mining season and on thrust times (which must be short c.f. the transfer semi-orbit), are too severe, then one needs to look for orbits that appear to match closely to low-thrust, spiral-out, spiral-in pattern, ie, low eccentricity.

\subsubsection{Arjunas and low-e Amors ("Arjuna-Type'):}

The "Arjunas", and some Amors, have very nearly circular orbits. There are also probably some as yet undiscovered asteroids, in a class as yet unnamed, whose aphelia are $<1.0 \mathrm{AU}$, and are of low eccentricity.

Such close, low eccentricity, low i NEAs, may be favourable for spiral, non-Hohmann returns; a characteristic of these trajectories is the 'softness' of the launch window for return; effectively there are little or no time constraints; one is free to set solar collector size to minimize processing time plus return transit time. However, because of the long synodic period, "those objects with semimajor axis approaching 1 AU exhibit increasingly longer gaps between direct ballistic opportunities, owing to the low relative motion between themselves and the Earth" (Niehoff, 1978).

Low-ellipticity targets, both outside Earth orbit (Amors) and overlapping Earth orbit (Arjunas) give much less concern about phasing and mining season duration. This is because much more of the orbit arc for can be used both mining and thrusting. The return transfer orbit may approximate continuous - thrust spiral; and multiple-return 
missions are more feasible provided total mission time remains short enough for NPV considerations.

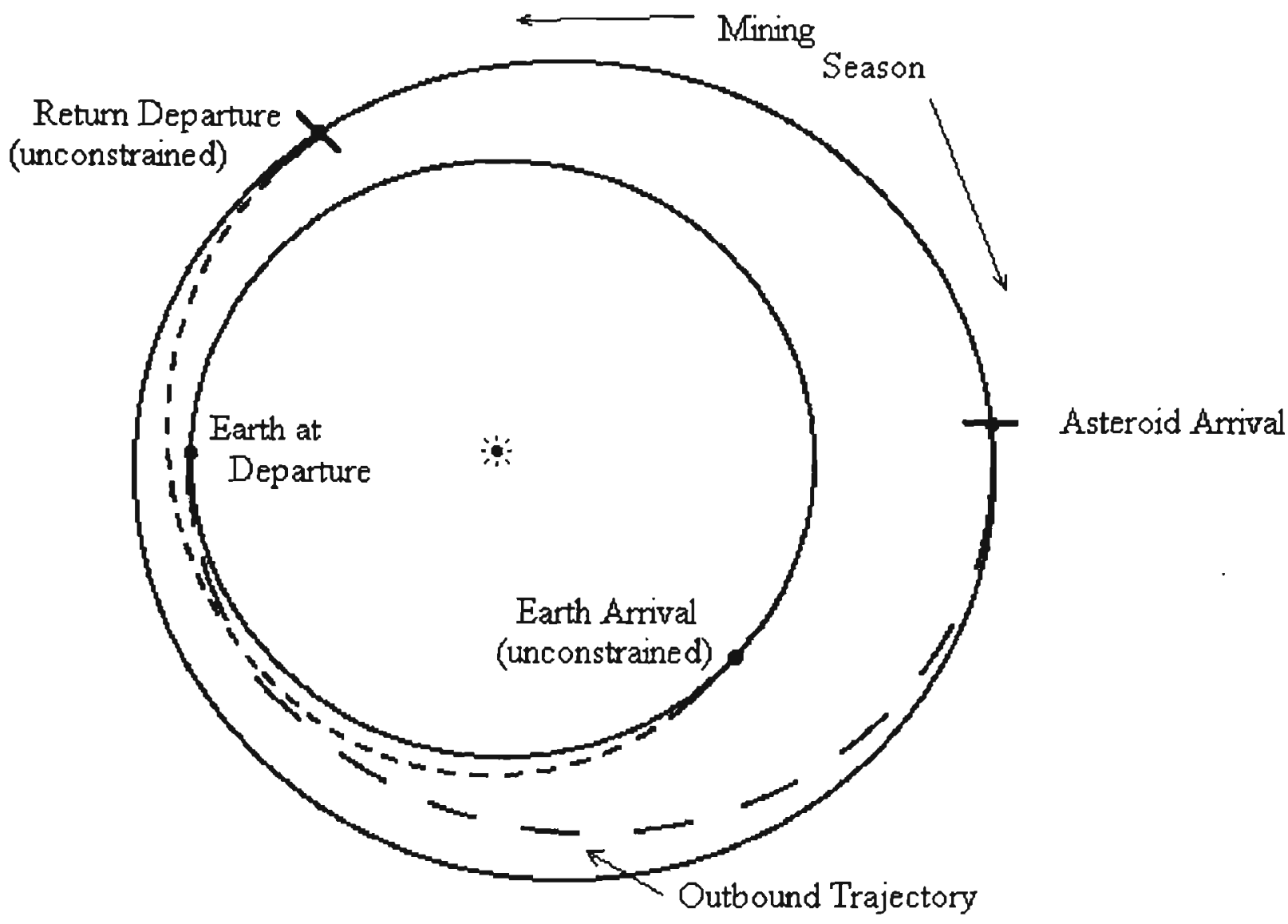

Figure 5.4 "Arjuna-Type" Mission

For low eccentricity targets with low eccentricity transfer orbits (eg Arjunas, but also some Apollos and Atens and Amors), Hohmann transfer is not essential; the major concern may be $\Delta v$ for inclination change.

Slow spiral return implies longer mining season, and hence less demanding specifications on mining, processing, and propulsion equipment, and on solar collector. Note that spiral return trajectories can be designed to deliver the payload at very small $\mathrm{v}_{\text {hyp }}$ (hyperbolic return velocity), because the spacecraft trajectory can be made tangent to the Earth's orbit. Such low v hyp implies easy capture into HEEO (Highly Elliptical Earth Orbit) by lunar flyby. 


\subsubsection{High-inclination, low eccentricity targets:}

The overriding characteristic of these missions is the need for high thrust during passage through the nodes. With low eccentricity targets, phasing with regard to perihelion / aphelion is not an issue, but inclination change can be a major impulse demand, $(\Delta v$ inclin $\cong 0.5 \times \mathrm{i} \mathrm{km} / \mathrm{sec}$.), so timing of mission phases with respect to Ascending / Descending Nodes may be important.

In this case, the timing and duration of the out and return trajectories will be determined primarily by the location of the ascending and descending nodes, rather than the location of the aphelion or perihelion, as in cases 1 and 2 (i.e. launch when Earth is at AN/DN). All configurations seem to suggest $\leq 180^{\circ}$ return thrusting, centred on a node; and $\leq 180^{\circ}$ mining season, centred on a node; and $90^{\circ}$ to $180^{\circ}$ outbound trajectory.

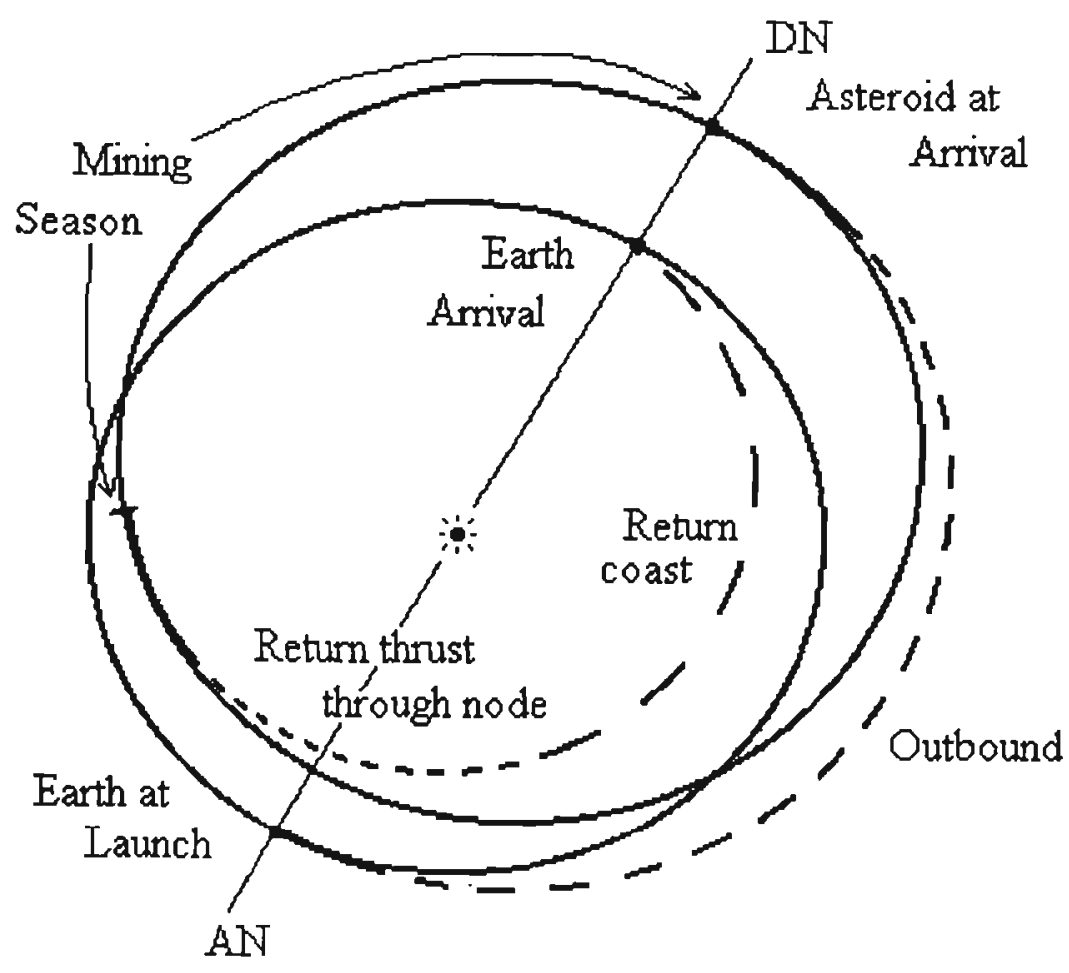

Figure 5.5 "High-i, Low-e" Missions

Note that perihelion missions in 5.2.2, 5.2.3, and 5.2.4 above may keep the target close enough to Earth to enable teleoperation rather than require machine autonomy. This may very well impact on probability of success. 
Note also that perihelion missions are shorter duration, and enjoy the advantage of better solar insolation. Both impact positively on NPV. But short mining season implies high mass throughput requirement, as does the higher propellant production requirement due to higher $\Delta v$ characteristic of the transfer orbit.

\subsection{Return to Earth Orbit Capture (LEO or HEEO)}

A major energy cost of the return mission is to decelerate the payload so as to achieve Earth-capture. There are various possibilities for reducing velocity from hyperbolic to a bound orbit upon return:

(i) rely on propulsive braking, using some of the Asteroid-derived propellant; this is simplest, but undesirable, as it reduces that which is available for sale. It is also most efficient in propellant use if done in an impulsive, high thrust manoeuvre, a system demand not otherwise addressed.

(ii) rely on aerobraking, using an Earth-fabricated, LEO-fabricated, or asteroidfabricated aerobrake. May be metallic or refractory silicate. The question is, how to fabricate an aerobrake on an asteroid, by remote means, using insitu resources?

(iii) use of a conducting tether for electromagnetic braking has been considered, but it does not give anywhere near enough retardation, either when considered as a passive conductor cutting the Earth's magnetic field (taken to average 0.1 gauss over an interception distance of $12,000 \mathrm{~km}$ ), or with an actively driven current of any reasonable size.

(iv) use lunar flyby to remove hyperbolic $\Delta \mathrm{v}$. This will naturally insert the returning craft into HEEO (Highly Elliptical Earth Orbit)! Navigation requirements must be met, to ensure the requisite low altitude pass over the Moon at the proper time in its orbit to provide maximum velocity loss. Note that gravitational capture exerts no stress on the payload package. However there is a tight constraint on the return "capture window". O'Leary quotes $\max 1.5 \mathrm{~km} / \mathrm{sec}$ for single lunar flyby.

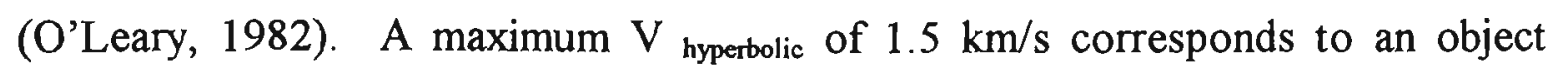
returning on a transfer orbit of $\mathrm{Q}=1.25 \mathrm{AU}$, from an Aphelion mining mission; 
and an object returning on a transfer orbit of $\mathrm{q}=0.83 \mathrm{AU}$ from a perihelion mining mission.

Thus, the most desirable targets for lunar flyby capture are those asteroids whose orbits are nearly tangent to Earth's orbit, and with aphelia less than 1.25 AU or perihelia more than $0.83 \mathrm{AU}$.
i.e., $q>0.83 \mathrm{AU}$,
a $>0.9 \mathrm{AU}$
or $\mathrm{Q}<1.25 \mathrm{AU}$,
$\mathrm{a}<1.25 \mathrm{AU}$.

Future detailed trajectory design will need to use mathematical and analytical tools described in Yen, 1984, ('mission opportunity maps'), and optimizer programs such as Science Applications International Corp's Trajectory Optimizer program (available from SAIC, Schaumberg., Illinois).

\subsection{Arguments against Multiple Trip Scenarios:}

The NPV calculations in Chapter $7 \& 8$ have been performed assuming a single return. Repeated returns to the target asteroid have not been considered in this work, because

(i) the high required IRR means that receipts following the first one are heavily discounted;

(ii) it is assumed that any later mission to the same target will be severely "offoptimum" compared with the first, to the extent that a different target will be preferable;

(iii) it is assumed that the operator will want to recover the remote miner and refurbish and upgrade it;

(iv) it is assumed that lessons learned after the first mission will dictate modifications to both the equipment and the mission planning.

Chapter 9 gives fully worked examples for each of these mission types. 


\subsection{Conclusions:}

(i) there are several mission types that can be identified, each with implications for length of mining season and total mission duration;

(ii) Earth-return hyperbolic velocity is a major mission $\Delta \mathrm{v}$ demand;

(iii) synodic considerations suggest that "multiple return" missions to a permanentlyemplaced mining facility are not competitive. 


\section{Chapter 6: Engineering Choices - Mining and Processing}

\subsection{Considerations in Space Mining}

The definition of "ore" depends on what can be recovered to sell or barter for profit. It is that which can be sold upon return to earth, eg Platinum Group Metals; or (the object of this thesis) that which can be sold for delivery into some orbit in space; or that which is not valuable to others, but which is valuable to the operator, as import replacement, or for process enablement.

\subsubsection{Mining in Near-Zero-Gravity conditions}

In order to perform mining operations on an asteroid or comet, one has to dock the mining equipment with it and "make secure". How? Note that gravity on a $10 \mathrm{~km}$ diam rock is about 1 milli-g; on a $1 \mathrm{~km}$ rock, gravity is about $0.1 \mathrm{milli}-\mathrm{g}$ (i.e., $0.1 \mathrm{~cm} / \mathrm{s}^{2}$ ). So there's no weight to provide stability. Also note that average rotation period is about 5 hours.

Anchoring is easy with rigid, competent, strongly bonded matrices - you can drive in pitons, glue or adhere to surface, or clamp against opposing surfaces. But it is likely to be very difficult with low strength or unconsolidated material. This may need very wide area anchoring, over an extended footprint, up to and including the approach of totally surrounding the target asteroid - wrapping it in plastic, so to speak.

Possibilities for securing to an asteroid are:

- $\quad$ tie the spacecraft down with a rope passing around the entire NEA

- $\quad$ drive in pitons - requires you assume the material is mechanically competent

- fire in harpoons or penetrators which resist extraction

- $\quad$ screw in large area augers or screw-plates - requires assumption that there is a regolith and it is loose enough and compressible enough for screw to penetrate (for screw-plate technology, for anchoring in low gravity, see Kloski (1995)).

- weld tie-downs into massive clasts of metal, ice, or solid silicate rock 
- use large area fluked anchors

- burrow completely into the regolith, somehow (e.g., using contra-rotating screws)

\subsubsection{Possible Extraction and Collection Methods}

Hard rock mining on the Moon can easily be extrapolated from Earth experience, but mining on asteroids will, because of the low gravity, require positive anchoring of the drill, pick, or cutting head, so as to generate adequate force against the rock, ice, or metal.

The reaction forces created by such operations as drilling or scraping may require to be spread over a very wide "footprint", if the regolith strength is low, and because of the milli-g gravity.

The mining method will depend on the material being sought. If regolith, the method will clearly be very different from that chosen if recovering solid metal; different again, if the "ore" is high in volatiles and ices. Loose material can be scooped, scraped, or shovelled. Friable but bound material will have to be broken or cut, or somehow disaggregated, before collection. Hard rock will require drilling, cutting, or blasting.

Containment will be important, because escape velocity for small asteroids may be of the order of $20 \mathrm{~cm} / \mathrm{s}$.

Frozen volatiles may be cut or melted at low temperature. Solid metal must be cut or melted at high temperature, or reacted at a lower one, eg. using vapour-metallurgical techniques of the Mond process, as have been proposed by Lewis and Nozette (1983) and Lewis, Jones, and Farrand (1988). 
Mining approaches will depend on the material:

loose regolith - scraper etc

competent silicate matrix - drill and blast or cut

silicates and ices or hydrocarbons - vaporization

silicate and metal - cut and crush

extensive metal - cut

- more exotic approaches may include carbonyl volatilization, or electrolytic release.

If it is necessary to break rock, then that requires that a force be exerted against the rock surface, either by impact or by pressurization or by static loading (eg impact of a pick, pressurization of a drill hole by an explosion, or static loading by the teeth of a roadheader or cutting discs of a tunnelborer). Classical percussion drills use the inertia (of the jumbo machine) or pneumatic pressure (of the airleg) to resist the Normal Reaction of the face being bored. Down-the-hole-hammer drills react against the inertia of the drill string and indirectly its friction against the side of the hole. Tunnelborers clamp against the already-cut tunnel walls.

In any operation, the mining machinery must be anchored to the asteroid surface, and the released material efficiently recovered.

\subsubsection{Surface mining}

Regolith mining on the Moon would, by analogy with sand and gravel pit operations on the Earth, use Front End Loaders and trucks, or Load-Haul-Dumps. Small quantities may be reclaimed by use of scraper/winch systems. Gertsch (1984) has proposed the classical three-drum slusher/scraper for lunar operations, because of its simplicity and low mass. None of this however appears to be applicable to asteroids, where the overriding considerations appear to be (i) very low strength regolith; (ii) zero gravity..

Note that in milli-g it is necessary to (i) ensure the scraper or shovel is held against the surface; and (ii) ensure that collected material is effectively retained within the collecting mechanism, and doesn't "float away". 
Thus, mining on low gravity bodies will require an approach which encloses the regolith being collected, eg by a screw conveyor or an enclosed drag chain conveyor, or uses a cactus or clamshell grab and gives positive displacement. An enclosed flail will also disaggregate and crush.

\subsubsection{Underground extraction}

There may be good reasons to use underground mining techniques, both on the Moon and when mining on asteroids:

(i) easier to generate reaction forces for cutting, drilling, or dragging (i.e., more "normal" technology)

(ii) the surface layer may be depleted in the desired material (e.g., volatiles at depth supposed to reside within Phobos or Deimos; or volatiles under a lag deposit in a dormant comet)

(iii) it may be easier to contain the cut or released material.

(iv) the resulting volume may itself be useful, e.g., for storage, habitat, or plant.

Underground mining on the Moon would probably use roadheader-type technology, with subsequent shotcreting to seal the excavated heading and enable it to contain an atmosphere.

Virtually all underground mining technologies could be used, but one should be chosen which uses minimum consumables, or none at all. One also should be chosen which does not require a large normal reaction force, and which has minimum impact on ground which is suspected to be weak and friable. (Even in milli-g, failures of ground will be inconvenient).

Note that the underground mining techniques are the only ones relevant for mining "massive" competent material, material which is not regolith. Hence they are the only techniques for use with regolith-free bodies, and in the case of needing to respond to an Earth-impact threat, they are necessary for placement of sub-surface nuclear explosives, desireable for best coupling of momentum from blast ejecta to the body. 


\subsubsection{In-situ extraction}

A particular case of underground extraction is, as noted by Sharp, Miller, and Gertsch (1993), and Kuck (1995), fluid extraction through drillholes. This is analogous to the Frasch process for melting and extraction of liquid sulphur from deep deposits using injected steam, and solution mining using a circulating solvent, as is practised in in-situ leach of uranium orebodies, and in solution extraction of salt deposits.

Kuck (1995) has listed the following benefits and risks of in-situ extraction:

\section{benefits:}

- $\quad$ simplicity and smaller mass of equipment

- no mining, transportation, crushing, grinding, separation, solid material handling, or tailings disposal to worry about

- the body itself provides the reaction vessel

- no power needed to crush, grind, etc.

- much less complicated

risks:

- loss of drilling and heat transfer fluid due to (a) blowout or intersection with large voids or fissures, (b) excess seepage into porous or loosely consolidated matrix, (c) insufficient volatiles "make" to replace this fluid loss

- $\quad$ incomplete separation of solids from return fluid

- $\quad$ plugging of equipt due to precipitation by sulphur or hydrocarbons

- $\quad$ plugging of matrix by fine solids, clays, etc.

- $\quad$ insufficient matrix permeability

This is simple technology, but may be threatened by loss of circulating fluid, due to porosity or permeability. Hence such an approach must provide for make-up fluid, preferably from the material being mined, or be able to seal the volume from within which the material is being extracted. In certain circumstances, e.g. use of steam in a permafrost deposit, this sealing may occur naturally. 
Mining is assumed to achieve $10 \%$ by processed mass recovery of volatiles from predominantly phyllosilicate and carbonaceous matrix regolith, or close to $100 \%$ recovery of water by drilling to extract primordial ice from an extinct comet core.

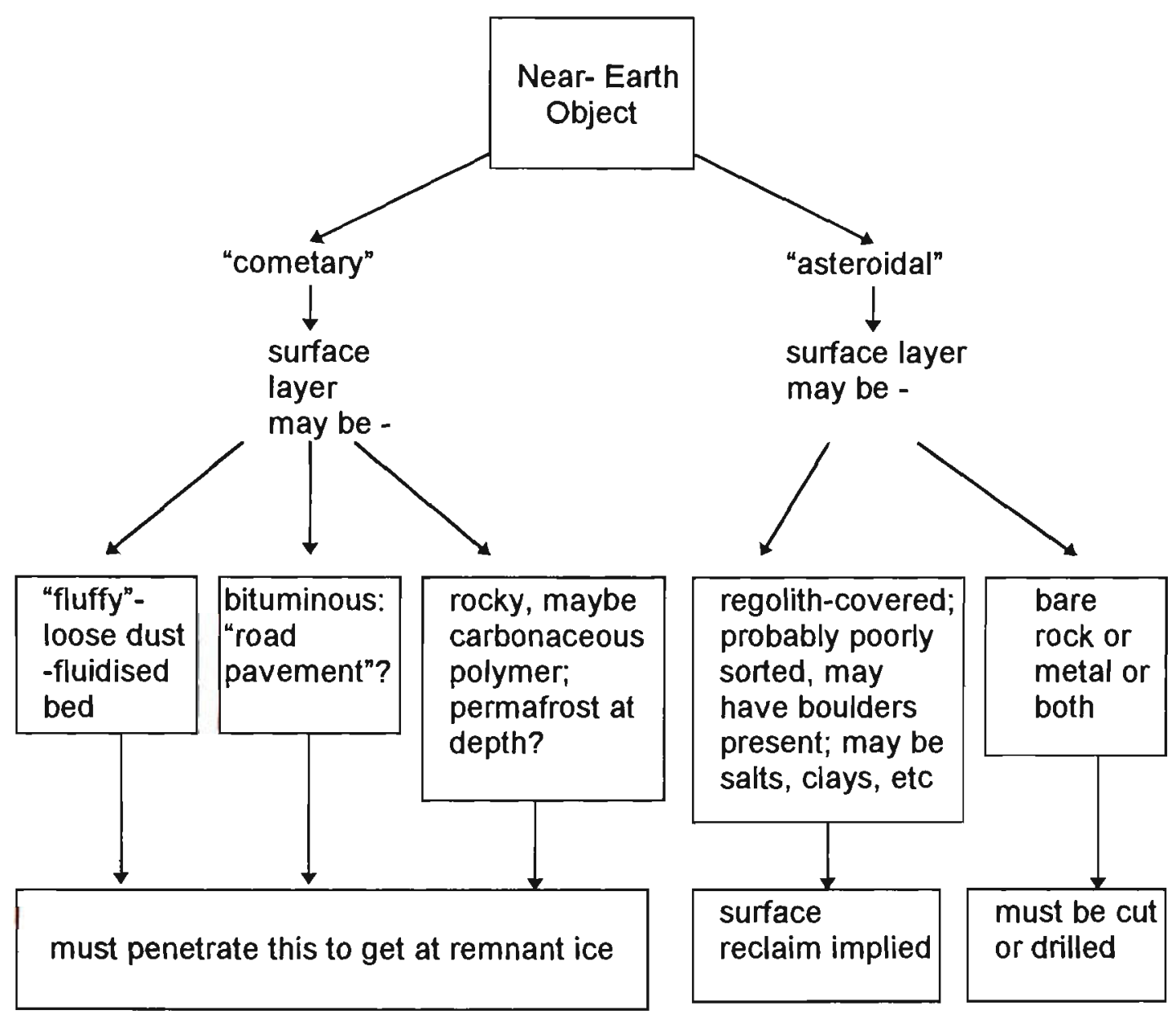

Figure 6.1 Mining Engineering Choices and Constraints 


\subsection{Metallurgical Processing / Beneficiation.}

These processes will depend on the material which has been collected, and the product(s) being extracted. Table 6.1, drawn partially from Burbine \& Binzel (1994), brings together the concepts presented below.

\subsubsection{Comminution:}

Most metallurgical separation processes commence with crushing and grinding so as to liberate the wanted particles or at least to obtain access to free surfaces of fine particles of the desired mineral. This is not true however in the cases of eg mineral sands processing, where the particles are already freed and the task is merely one of separation; or extraction of salt, sulphur, uranium or gypsum using surface or insitu dissolution methods. Liberation of individual valuable grains from matrix material may in some situations be quite easy as, depending on the details, it may be possible to sort directly from the regolith eg using a magnet for $\mathrm{NiFe}$ grains (Lewis proposes a magnetic rake), or remove as a liquid or vapour (eg various ices, or metal carbonyl as in the Mond process).

If the desired material is all in the big lumps, then crushing is not needed, in order to obtain recovery; simply identify and separate. This may be the case with, for example, lumps of ice in cometary bodies, or alternatively, lumps of silicates for rejection; and in the case of lumps of metal in the regoliths of rocky bodies.

In this case, mining and beneficiation of material from a loose, coarse, heterogeneous regolith may most simply be done by "manual sorting" using a dextrous 3 or 4-fingered robot manipulator for selective collection of lumps of a signature matching that retained by the robot and defined by a discriminating expert system.

Note alternatively, that the valuable resource may be in the "fines", and there may be "enough" fines available in the regolith to dispense with crushing and grinding, requiring merely sorting, e.g. by sieve or cyclone, to separate and collect the desired feedstock. 
If the material being handled is regolith and if the desired fraction is in the fines, then the process may require little or no comminution, or only very gentle disaggregation, followed by size separation.

In this case, adequate liberation of volatiles, especially, might not require grinding; in which case, this operation should obviously be deleted!

However, assuming that it is necessary to crush and grind, note that particle separation is then desireable to provide one stream of material of enhanced grade for further treatment, and another stream (tailings) for rejection.

\subsubsection{Separation}

Separation of fine particles can be by any of the following:

- electrostatic field, e.g. high voltage plate separators;

- magnetic field, e.g. cross-belt magnets;

- density differences e.g. air- or hydro- cyclone;

- vaporization / condensation

- sublimation / deposition, e.g. the Mond process)

- sizing / sieving

\subsubsection{Processing: influence of the desired product type -}

(a) Metal

- if present as loose grains - electrostatic or magnetic separation is needed

- if present as macroscopic lumps in a silicate matrix - crushing then sieving

- if present as interconnected dendrites with minor silicate - carbonyl separation

- if present as continuous mass - cut off big slabs (?)

(b) Volatiles

- $\quad$ if present with minor silicate ('dirty iceberg' - melt or cut off slabs 
- $\quad$ if minor component (icy mudball - permafrost) - drill into, vaporize and distill

- if present only in chemical combination (eg, water of hydration, ammonium or carbonate compounds), then severe heating required (e.g., to $800 \mathrm{~K}$ )

\section{(c) Hydrocarbons}

- $\quad$ if present with major silicates, then heat and distill.

Vapour phase processes will imply:

heating and volatilization, probably with a carrier or reactant gas

collection of gas, vapours, and entrained solids

separation of entrained solids

condensation of vapours collectively or sequentially

recovery of gas, reheating and re-injection

\subsubsection{Processing - influence of plant feedstock:}

- $\quad$ is it mixed aqueous and organic liquids, e.g., from drill hole mining of extinct comet? - in this case, oil-water separator technology is needed: see the oil industry for useful technology.

- $\quad$ is it mixed liquids as above, with gases and vapours, i.e., emulsion or foam? - again see the oil industry.

- $\quad$ is it various sizes of solid particles (coarse to fine, soft to hard, siliceous or metallic, reactive or not) in gases; then the experience and techniques of the dry process metallurgist are called for- see the mineral sands people.

- is it solids, liquids, and gases? - then one will need to heat, separate by cyclone, and treat separately. Filter screens can be expected to blind. Perhaps review material handling in food industry. 


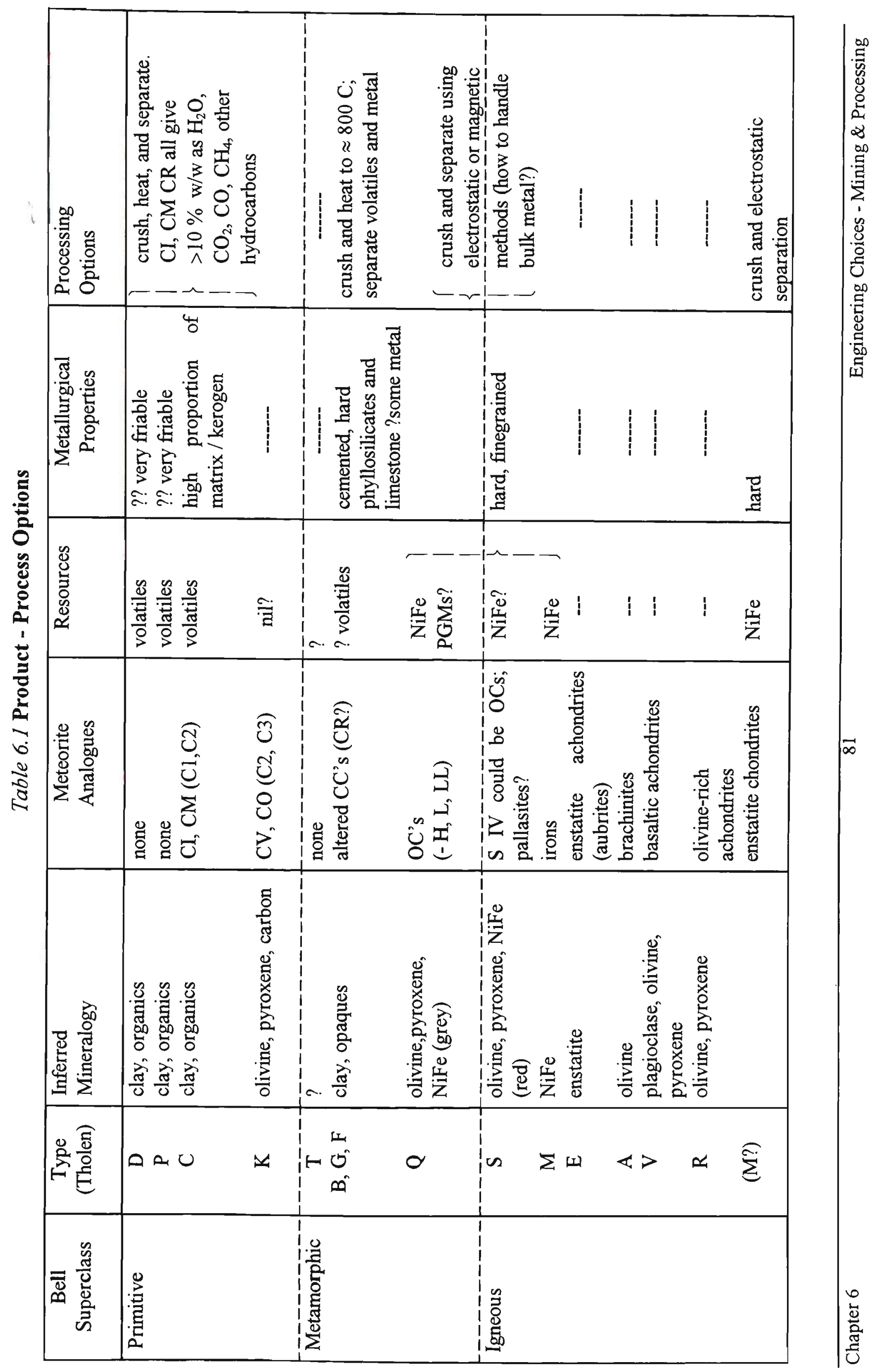




\subsection{Feasible Products and Processing Methods}

According to Lewis (1993), the prime targets should be the asteroids which are analogues of the carbonaceous chondrites, initially for their volatiles, for the following reasons:

(i) they are apparently plentiful in number

(ii) they contain $\mathrm{H}_{2} \mathrm{O}, \mathrm{CO}_{2}, \mathrm{CO}, \mathrm{CH}_{4}, \mathrm{NH}_{3}$, etc.

(iii) they contain metal (albeit mainly in oxidized form; but readily reduced)

(iv) the metal phase should contain high grade PGMs

This implies earthmoving or burrowing into regolith; heating to extract water and other volatiles; perhaps further heating to reduce metal oxides to metal and produce $\mathrm{CO}_{2}$; extraction of metal by, e.g., electrostatic or magnetic means, or by carbonyl volatilization.

Note that this is going to require quite a bit of solar heating; it is energy intensive. Thus the question arises, what is the status of lightweight solar mirror technology?

The same solar mirror can drive the solar thermal rocket for material return, provided the power requirements are similar.

A different approach is taken by Kuck (1995) who recommended targeting the dormant comets, because it is only with them that it is unequivocally clear that one can obtain water; and he claims that the greater required mass of mining and processing equipment for extracting volatiles from the asteroids militates against their early viability. The down-side of Kuck's proposal is that almost all of the probable targets for his scenario are in orbits which are much less accessible, energetically speaking, than the potential targets that Lewis can propose. 
Zuppero is also supportive of comet mining, but has specifically recommended as his target 1979VA Wilson-Harrington, and has proposed a nuclear thermal rocket using recovered water as the propellant (Zuppero, Whitman, \& Sykes, 1993; Zuppero, 1996).

Meinel \& Parks (1985) forego In -Situ Propellant Production. But this can only be supported by limiting target choice to the most energetically accessible asteroids, and by severe restriction on returned mass, and by assuming market is in high earth orbit, i.e. GEO or HEEO.

Kargel has proposed mining the ordinary chondrite analogue asteroids for the PGMs.(Kargel, 1994). However, precious metals are likely only to be a small byproduct in any near-term project, and only become viable as a primary product on its own at a scale that implies very large impact on the earth's supply of Platinum, and a very large inspace mining operation.

The details of the beneficiation process to be chosen obviously depends on the material being recovered. If it is fines from which it is hoped to extract Fe-Ni metal sand plus volatiles, then one might consider the following conceptual flowsheet (adapted from O’Leary, 1982).

\section{Conceptual flowsheet, from O'Leary:}

1. material collection, for example, by scraper or screw conveyor;

2. pressurization, to $\sim 0.01$ atmosphere, to enable pneumatic handling;

3. comminution, to $\sim 0.2 \mathrm{~mm}$, to release $\mathrm{NiFe}$ fines and volatiles;

4. sizing, for example, by dry cyclone, for effective separation;

5. magnetic separation, by cross-belt magnets to collect metal grains, magnetite, and ilmenite;

6. heating, using solar furnace, to $\sim 500 \mathrm{C}$ to extract vapours and gases;

7. separation of gases and remaining solids, by cyclone;

8. condensation of gases and vapours in cold traps (shaded vessels);

9. recirculation of permanent gases for pneumatic handling. 


\subsection{Equipment Considerations}

Here are addressed some aspects of likely equipment mass and power requirements.

\subsubsection{Grinding and crushing}

Assuming that the material does need comminution, there are several possible equipment choices.

Energy requirements are estimated using the Bond Work Index; this is basically an indication of ore hardness. It is defined as the specific work (in kilowatt hours per tonne) required to reduce particles from infinite size to 100 microns. Average Work Index for industrial feedstocks appears to be 10 to $15 \mathrm{~kW}$-hr/tonne.

It is worth noting that about $99 \%$ of energy input into crushing and grinding goes into heat, with only $1 \%$ going into the energy of formation of new surfaces.

One can readily visualise a conical gyratory "grizzly" with external annular conical screens for scalping oversize and delivering fines for further separation. Note that screens must be non-blinding and capable of being cleared by reverse flow cleaning.

Roll crushers have low energy requirement, of order of $0.5 \mathrm{~kW} /$ tonne.hr., and give a low production of fines.

Fluid energy mills: An example is the "micronizer", which gives a very fine grind, and 1 metre diam model handles 2 tonnes /hr. (SME Mineral Processing Handbook).

Hammer mills are used as coal pulverizers; hot gas can be used to dry the coal during pulverizing, from $8 \%$ moisture to $1 \%$ moisture. 


\subsubsection{Materials Handling}

1. Solutions here are not altogether obvious, given:

(i) the process takes place in zero-g;

(ii) the material(s) are ill-defined and likely to be extremely poorly sorted in particle size, and chemically heterogeneous, and possibly mutually reactive when heated.

2. Low pressure pneumatic transport was suggested by O'Leary, referenced above. As in the case of Kuck's Frasch-process-style extraction, it is important to know whether you can supply or generate make-up fluid to replace losses due to leakage, reaction, etc.

Again as in the case of Kuck's process, if one were mining for volatiles, you would certainly expect to be able to generate adequate make-up gases such as $\mathrm{CO}, \mathrm{CH}_{4}, \mathrm{~N}_{2}$, $\mathrm{CO}_{2}, \mathrm{HCN}, \mathrm{NO}_{2}$, etc. There are various advantages with pneumatic handling: good heat transfer properties; minimise reliance on mechanical equipment; easy release, entrainment and capture of mined volatiles, by use of a cold trap (which as pointed out by O'Leary, can be as simple as a shaded storage container).

Note that the extraction "head" must be gas tight else material will be lost; a "guard ring" approach to design is needed.

3. Steam ejectors can be used as gas pumps or pressurizers:

- $\quad$ they can achieve compression ratios of up to $10: 1$;

- they can handle gas with entrained dust or condensate;

- $\quad$ they have no moving parts, hence no maintenance requirement. (see Perry's Chemical Engineer's Handbook, McGraw Hill).

4. Pneumatic conveyors "can be used for free-flowing materials of almost any particle size up to approx $6 \mathrm{~mm}$ at rates over 10 tonnes per hour. Typical pressure loss is about 0.5 atmosphere." At $20 \mathrm{t} / \mathrm{hr}$., transporting $0.5 \mathrm{gm} / \mathrm{cc}$ suspension, $150 \mathrm{~mm}$ 
diam pipe is needed. (see Society of Mining Engineers Mineral Processing Handbook, 1985).

5. Screw conveyors for material handling: should work effectively in zero-gravity. They are very robust, accept coarse material, and for material of bulk density close to $1 \mathrm{gm} / \mathrm{cc}$, the power requirement is approx $1 \mathrm{~kW}$ per 10 tonnes $/ \mathrm{hr}$ tranported through 10 metres of conveyor flight.

6 Rotary feeder /star wheel feeder can hold off 1 atmosphere pressure.

\subsubsection{Heat Processing}

Both "Kuck Process" and Carbonaceous Chondrite Devolatilization rely on heating for product recovery; here the power requirement for the minimum-scale project concept is discussed.

\subsubsection{Melting Ice}

Assume it is planned to extract (via Frasch process for example) 5000 tonnes of $\mathrm{H}_{2} \mathrm{O}$ ice from an extinct comet core, within a period of 6 months. This throughput implies a processing rate of $\sim 250 \mathrm{grams} / \mathrm{second}$.

Heat $=$ Mass $\times$ Specific Heat of Fusion

Power required is $=2.5 \times 10^{2} \times 4.2 \times 80$ joules $/ \mathrm{sec}=80 \mathrm{~kW}$;

If the water needs to be volatilized, then the power required becomes $\cong 800 \mathrm{~kW}$.

Since solar insolation at $1 \mathrm{AU}$ is $1 \mathrm{~kW} / \mathrm{m}^{2}$, the required mirror size is then either $80 \mathrm{~m}^{2}$, or $800 \mathrm{~m}^{2}$. 


\subsubsection{De-volatilizing carbonaceous chondrites}

Assume that it is planned to 'cook out' approx $10 \%$ of the mass of a CI or CM chondrite (and /or reduce another $10 \%$ of the mass ( $\mathrm{FeO}$ to $\mathrm{Fe}$ ) if $\mathrm{CR}$ chondrite). Note that it is necessary to heat to approx $800 \mathrm{C}$ in order to extract $\mathrm{H}_{2} \mathrm{O}$ from talc and other phyllosilicates (Ganguly \& Saxena, 1989). The temperature required to extract $\mathrm{H}_{2} \mathrm{O}$ from gypsum is (depending on the rate of heating) 500 to $600 \mathrm{C}$.

No quantitative data exists for volatiles release as a function of temperature and heating rate, for meteoritic materials, and there is very little information on the effect of heating rate even for simulants, such as gypsum, epsomite, calcite, dolomite, clays, oilshale, and combinations of these materials. Extraction of volatiles has not been studied at all from the point of view of yield determination, experimentally.

There is however some information available, as an aside, collected during stepwise heating experiments done for the purpose of identifying hydrocarbon species in Allende, Murchison, and Murray meteorite samples; and from experiments done for the purpose of looking at isotopic ratios of hydrogen, oxygen, and nitrogen; but it is incomplete and it was not performed to address the question of volatiles yield vs temperature vs time for various carbonaceous chondrite types.

The stepwise heating experiments done by Levy, Studier et al, Kerridge, Robert and Epstein, Villieras et al, and others, all tend to indicate that up to $10 \% \mathrm{H}_{2} \mathrm{O}$, and possibly up to $5 \% \mathrm{CO}_{2}$, may be released These are however, underestimates, because in most of the above cases, volatiles that were released at temperatures below $150 \mathrm{C}$ or below $200 \mathrm{C}$ were not reported, or were not accounted for, were treated as nuisance material, and pumped to waste, or collected on $\mathrm{KOH}$ and discarded. Ganguly \& Saxena (1989) have done calculations which suggest up to $30 \%$ by weight of the carbonaceous chondrite material could be released on heating, but this has not yet been tested. 
Experimental results (Levy, 1973; Kerridge, 1985) and theoretical calculations (Ganguly \& Saxena, 1989) both suggest that the release reqires that the chondritic material be heated to quite high temperatures (variously $400,600,800$ or 1000C).

CR chondrites have significant calcite / dolomite; we know therefore that they may be heated to extract $\mathrm{CO}_{2}$.

Autoreduction of $\mathrm{FeO}$ with Carbon will depend on the mineral grains being in close contact and at high temperature. There is indication of autoreduction occurring in some chromatograms of Levy et al, at $600 \mathrm{C}$. It also appears to be occurring in the pyrolysis experiments of Robert \& Epstein, 1982.

However, in neither these nor other possible cases was it commented on, because it was not what the experimenters were interested in: they were not looking for a resourceextraction process.

As a result, Lewis and Hutson's assertion that "up to $40 \%$ by weight is extractable volatiles" is as yet untested by experiment, although clearly plausible, based on abundances and on the circumstantial evidence from the above heating experiments. Ganguly has experimentally shown a $3 \%$ water release from talc on its thermal decompositon to enstatite and quartz. He has also indicated good theoretical reasons to expect similar yields from thermal decomposition of other phyllosilicates.

So, a reasonable assessment of the literature reviewed is that if one wishes to extract water and carbon dioxide from $\mathrm{CI}, \mathrm{CM}$, or $\mathrm{CR}$ material then one has to heat it to (probably) about 600 to $800 \mathrm{C}$. If it is desired to obtain $\mathrm{CO}_{2}$ by autoreduction of intimately mixed $\mathrm{FeO}$ and $\mathrm{C}$ matrix in $\mathrm{CI}, \mathrm{CM}$ material, then it must be heated to approx $800 \mathrm{C}$.

Assume for a process restricted to volatiles production, i.e., no metal production, a temperature requirement of $500 \mathrm{C}$, and a volatiles recovery of $10 \%$ of the feed mass. 
What is the heat capacity of common solids?

\begin{tabular}{|c|c|c|c|c|}
\hline $\mathrm{CaCO}_{3}$ & $80 \mathrm{~J} / \mathrm{mole} . \mathrm{K}$ & M.W. $=100$ & hence & $0.8 \mathrm{~J} / \mathrm{gm} . \mathrm{K}$ \\
\hline $\mathrm{CuSO}_{4}$ & 100 & 160 & & 0.6 \\
\hline $\mathrm{SiO}_{2}$ & 50 & 50 & & 0.83 \\
\hline
\end{tabular}

- so $1 \mathrm{~J} / \mathrm{gm} . \mathrm{K}$ appears appropriate; note that water is $4.2 \mathrm{~J} / \mathrm{gm} . \mathrm{K}$.

At 12 tonnes per hour feed rate (to give 5000 tonnes of water after 6 months at 10\% recovery), and $\Delta \mathrm{T}$ of $400 \mathrm{C}$, the implied power requirement is $1.5 \mathrm{MW}$, in the absence of heat reclaim/ recuperator / heat exchanger. Note that this is only about twice the power requirement for volatilization of ice at the rate giving same volatiles production rate.

\subsubsection{Further heating and processing considerations}

What particle sizes do we need for liberation of $\mathrm{H}_{2} \mathrm{O}, \mathrm{CO}_{2}$, etc., on heating? -and what energy is required to grind to this size, if indeed grinding is required?

What heat energy is required to heat this sized fraction to say $600 \mathrm{C}$ ? -and what heat reclaim can be obtained?

It seems intuitive that release will be aided by reducing the particle size. However, Ganguly suggested that the dehydration reaction is adversely affected by too small a particle size, because that inhibits nucleation of the new species; however, he expects this to occur only at sub-micron sizes. This being the case, Ganguly would suggest that the production rate would be found to increase with reducing particle size, down to this small diameter, then reduce as size decreases below the optimum for nucleation, the optimum being perhaps at a micron or so.

However, a paper on Brucite decomposition indicated best size for speed of reaction to be at 150 microns; my own rough experiments on dehydration of gypsum showed little 
effect of particle size. Thus there may not be any need for fine grinding at all, with particles up to even 500 microns or so potentially processable with no great loss of yield.

It is likely that some of the engineering rules of thumb regarding sizing, throughput, mass, and heat transfer relating to fluidised bed reactors and pneumatic dryers may be applicable. For example, Fluidised Bed Reactors:

- $\quad$ provide extremely good heat transfer performance;

- $\quad$ are well understood if dilute phase flow regime; this holds if mass of particles is less than 5 times the mass of the fluid $\left(\mathrm{m}_{\mathrm{p}}<5 \mathrm{~m}_{\mathrm{f}}\right)$.

- Particle diameter should generally be between 60 micron and $2 \mathrm{~mm}$; i.e., not necessity for fine grind. $\left(60 \mu \mathrm{m}<\mathrm{d}_{\mathrm{p}}<2 \mathrm{~mm}\right)$.

- Gas cyclone can be used to return oversize particles to the FBR, or to separate and dump.

Pneumatic conveying dryers "are suited to free-flowing powders and granular, crystalline, or fibrous solids" (Perry's Chemical Engineers' Handbook). They may also be set up to grind the feed at the same time.

Note retention time of particles in hot zone is very short - a few seconds only).

Volumetric heat transfer is in order of $2000 \mathrm{~J} / \mathrm{m}^{3} \mathrm{~K}$. Thus, if $\sim 1 \mathrm{MW}$ is required, this implies heating / grinding chamber needs to be about $1 \mathrm{~m}^{3}$ in volume. (This can be reduced by heating of shell to add radiant heat input).

Typical products handled in pneumatic conveyor-dryers are (Perry's, p20-54): 


$\begin{array}{lccr}\text { material } & \text { initial moisture } & \text { final \% } & \text { rate (k } \\ & & & \\ \text { coal }(1 \mathrm{~cm}) & 11.5 \% & 1.5 & 1200 \\ \text { kaolin } & 10 & 0.5 & 120 \\ \text { clay (ball) } & 25 & 0.5 & 60 \\ \text { gypsum } & 25 & 5.0 & 50 \\ \text { silica gel } & 50 & 10.0 & 80\end{array}$

\subsubsection{Masses and throughputs of mining and processing equipment}

Materials collection:

Front End Loader: at 1 bucket per minute with a bucket load of 5 tonnes and machine mass of 10 tonnes, $f=720 \mathrm{~kg} /$ day per $\mathrm{kg}$ equipment

Scraper/winch: at 600 tonnes per day, with winch plus scraper mass of 0.2 tonnes, $\mathrm{f}=3000 \mathrm{~kg} /$ day per $\mathrm{kg}$

Grinding/separation: depends on type: fluid energy mills probably mass approx $50 \mathrm{~kg}$, unmodified; and appear to require about $10 \mathrm{~kW}$ for $500 \mathrm{~kg} / \mathrm{hr}$ throughput; so $\mathrm{f}=250$

Impactors/hammermills: mills massing 100 to $200 \mathrm{kgs}$ give 2 to 5 tonnes per hour, hence $\mathrm{f}=500$. Coal pulverizers are generally hammermills and the feed is often direct -heated with hot gas to dry coal from $8 \%$ moisture to $1 \%$.

Generalising and extrapolating from the above, $f$ (daily throughput/mass of equipment) may be approx $=200$; hence to mine and process chondrite material at 300 tonnes per day should require approx 1.5 tonnes of equipment.

This will give (according to the assumptions) 30 tonnes volatiles / day, and therefore a payload plus propellant cargo after 180 days of 5000 tonnes. 


\subsection{Process Flowsheet Concepts}

What:

1. collection

2. discard lumps

3. pressurize and fluidize soil

4. grind and heat

5. separate gases from solids

6. heat recovery $\&$ depressurize solids

7. discard solids

8. condense product vapours

9. store as ices

10. repressurize carrier gases
How:

by augers or scoop arms

by dimensions of collector or by annular 'grizzly'

rotary valve or screw feeder; gas ejector fluid mill or impactor / hammer mill dry cyclone or baghouse some sort of gas expansion engine some sort of slinger / ballistic ejector adiabatic (heat engine) expansion into storage bag radiative cooling of storage bag mechanical compressor driven by 6 or reheat 8; and solar collector heat exchanger

See Fig 6.2 over page. 


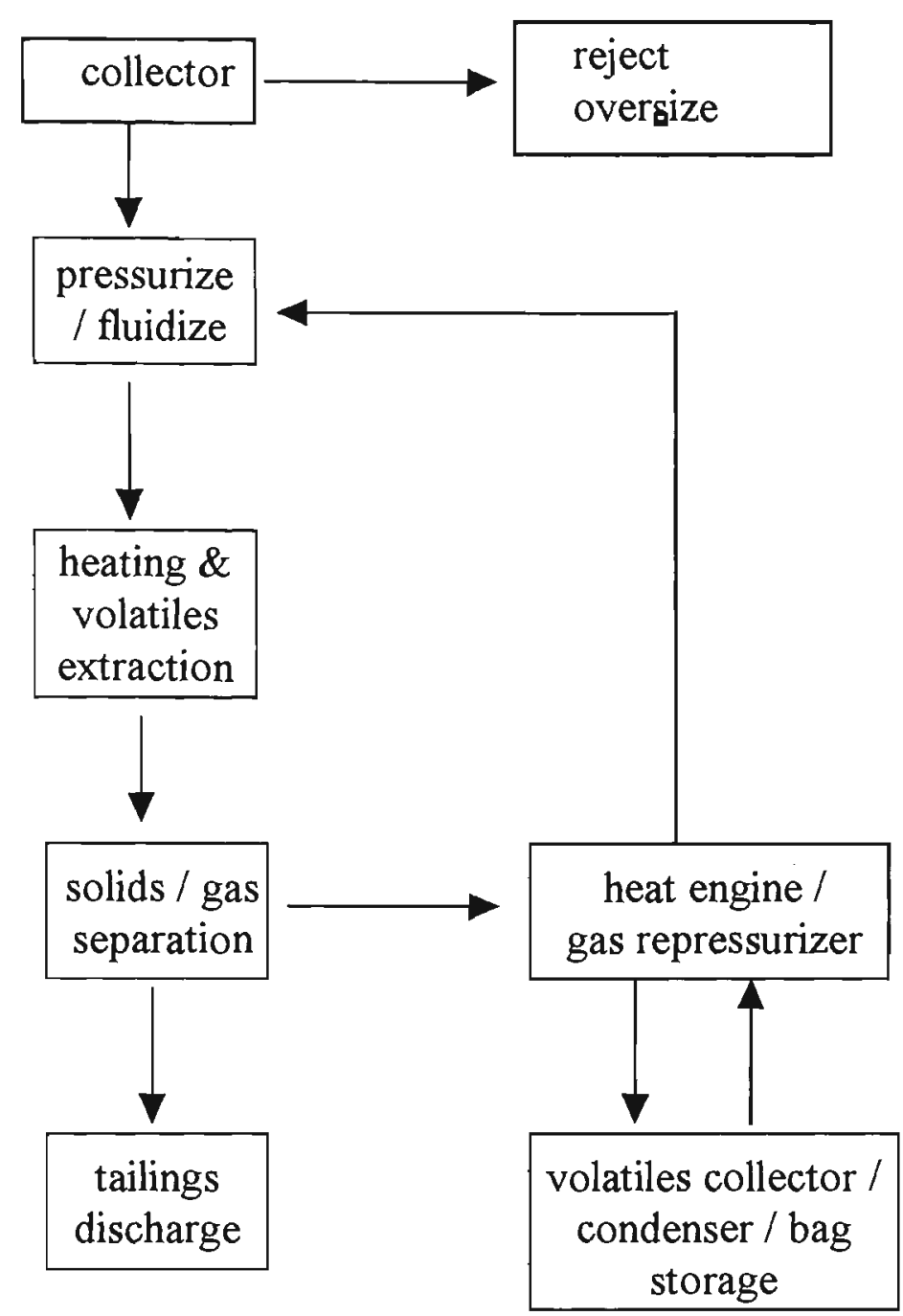

Figure 6.2 Conceptual Process Flowsheet for volatiles extraction from Carbonaceous Chondrite-type Asteroidal Regolith 
Notes on Flowsheet

This flowsheet is intended to extract volatiles (primarily water) from carbonaceous chondrite and hydrated silicate materials, found loose in asteroidal regolith.

Pressurization is necessary to enable gas-pneumatic handling of the solids, and for efficient heat transfer.

Rotary valves can hold off 1 Atm $(100 \mathrm{kPa})$. Screw conveyors are also capable of supporting substantial backpressure.

Grinding and heating duty should be integrated and simultaneous, because both require adequate residence time and fluid bed conditions are good for heat transfer.

Grind size should be kept as coarse as possible. to reduce grinding work requirement and to ease solid - gas separation, and/or to minimize blinding of filter cloth (say $50 \%$ passing 200 micron or coarser).

Temperature needs to be raised to approx $600 \mathrm{C}$ to ensure dehydration.

Gases must be cooled to (if possible) $-50 \mathrm{C}$; this should be partly by expansion through a heat engine; similarly, initial reheat after condensation of volatiles should be by mechanical compression, rather than heat exchanger; recompression being necessary for reuse as carrier gas, and heat exchangers being massive items.

Other issues:

It is necessary to address the mass of the heat engine and compressor, or steam ejector, or diffusion pump, for recirculation of the heat transport and grains-transport gases. It is also necessary to address the mass of the filter sock upstream of or internal to the volatiles collection and condensation bag. 
In the case of the in-situ drilling and melting scenario, the compressor requirement is (say) $0.5 \mathrm{~kg} / \mathrm{sec}$ of gas at (say) 4 atmospheres $(400 \mathrm{kPa}$ ), and this implies about $2 \mathrm{~kW}$ power requirement. Such a pump might mass 10 or 20 kilograms.

In the case of the mining and processing of regolith, there is a larger power requirement, mainly because of the order of magnitude greater throughput required, so in this case, the heat engine/compressor will mass (say) 100 to $200 \mathrm{~kg}$.

\subsection{Kuck Process Option.}

If one can be sure that the body to be mined is a comet core with remnant deep or shallow ices (under a non-volatile insulating layer that may be fluffy or rocky, or bituminous) then one would tend to choose Kuck's modified Frasch process for combined mining/processing. (see Figure 6.3 below).

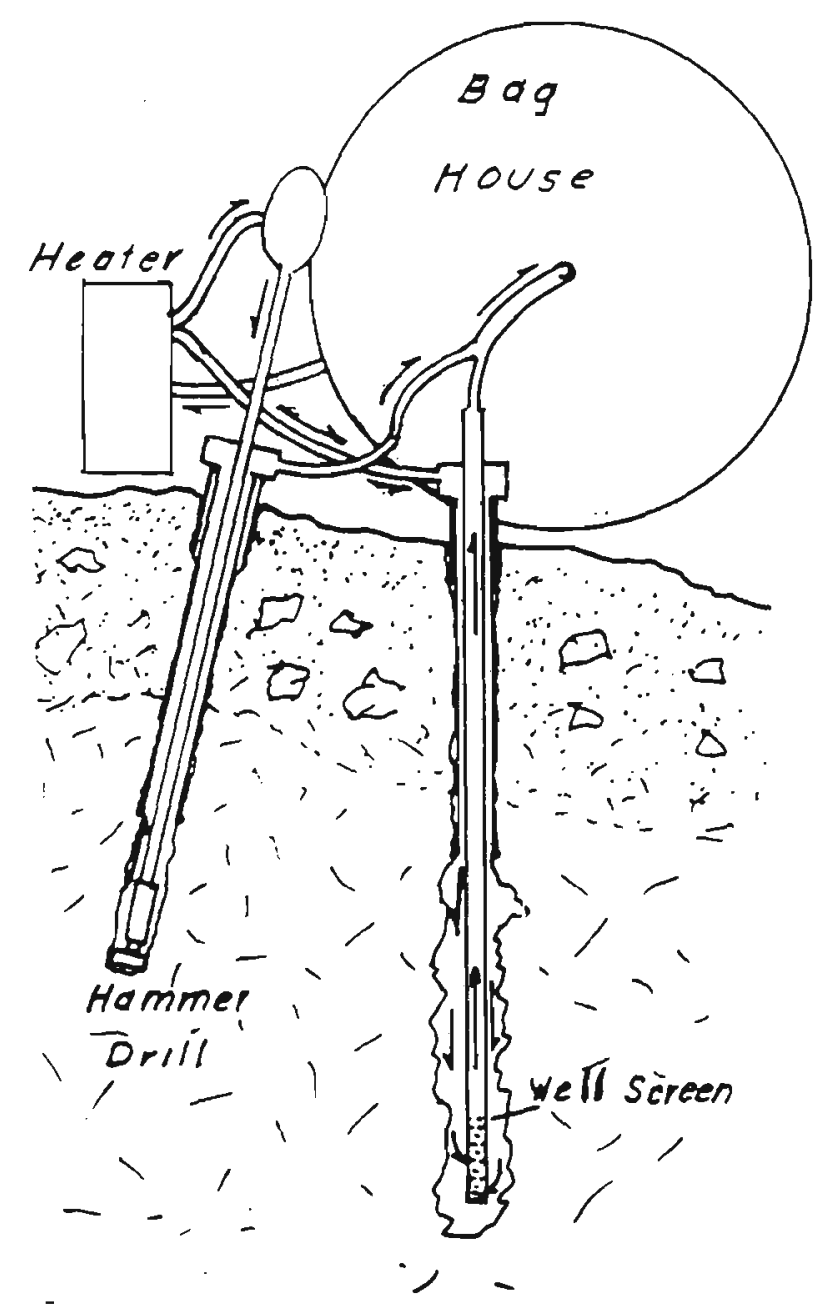

Figure 6.3 Drilling to recover volatiles from comet core (from Kuck, 1995) 
Note that we can only surmise as to the nature of the lag deposit or detrital cover layer. Lewis suggests that it may be as uncompacted and strengthless as fluff.

Kuck's process requires at least enough competence or strength in the surface layers to anchor the drill to, and to cement the wellcasing to. A poorly sorted rocky armour of 3 to 5 metres thickness with bituminous / tarry infilling would be almost ideal for this approach. Sandvik use the 'Tubex' method to drill into sanitary landfills and permafrost ground, and this could be adapted for the duty envisaged (Kuck, pers.comm. 1996).

Lewis's picture of a fluffy covering would be totally unsuited to a Kuck-process extraction rig, inasmuch as the drill rig relies on the strength of the surface non-volatile layer to provide reaction forces and anchoring forces, and to provide a gas-tight seal within which the volatiles can be fluidised.

Should the comet core be covered by "fluff" then the mining equipment will have to cut or melt into the exposed near-surface ice layer so as to form a seal.

The treatment of the product which has been fluidized depends on whether it is gas/vapour (and dust), liquid (and dust) or gas and liquid and dust. There may also be contaminant salts. Such multi-phase flow is highly likely to cause clogging in all but the simplest of processes.

It should be noted that the circulating species could be :

$$
\begin{aligned}
& \text { mobilising gas }\left(\mathrm{CO}_{2} \text { or } \mathrm{CO} \text { or } \mathrm{N}_{2}\right) \\
& \text { volatilized gas }\left(\mathrm{CO}_{2}, \mathrm{CO}, \mathrm{NH}_{3}, \mathrm{HCN}, \mathrm{CH}_{4}, \mathrm{C}_{2} \mathrm{H}_{6}, \mathrm{H}_{2} \mathrm{~S}, \mathrm{SO}_{2}\right. \text {, etc) } \\
& \text { vapours capable of easy condensation }\left(\mathrm{H}_{2} \mathrm{O}, \mathrm{C}_{6} \mathrm{H}_{14} \text {, etc }\right)
\end{aligned}
$$

silicate dust, carbon 'polymer' dust, inorganic salts, bituminous fines.

The process proposed by Kuck assumes collection of all particulates, along with the volatiles, so as to simplify the process flowsheet. This is only allowable if you believe that solids will be but a small proportion of the total collected mass. 
Otherwise, very simple separation of the dust is the first essential process step, and must occur at a temperature above the condensation point of the most easily condensed, highest condensing, vapours.

Simple cyclone gas cleaners are readily adaptable to zero-g. So are baghouses, but blinding must be considered to be a major problem.

Various gas cleaning and condensation steps may be necessary, with the permanent gases being re-compressed, reheated, and re-injected for further volatiles recovery.

\section{Items to be addressed in engineering a design for the Kuck process are:}

- what total volatiles payload should one aim for? (a figure of 1000 tonnes delivered into LEO is suggested elsewhere in this thesis)

- what heat input is needed to melt (or melt and volatilize) the required mass of payload and propellant?

- what hot gas flowrate would be desireable to do this? - and at what pressure?

- what solar collector size is therefore required?

- what radiator capacity is needed, to condense volatiles and dump heat engine waste heat?

- what is the estimated total mass of plant?

Note: if we do not volatilize, but merely melt, the desired material, then a gas-cleaning step would tend to reject product. Thus, a "melt only" Kuck process is restricted to a scenario in which (i) the total mass proportion of dust is low; and (ii) the presence of dust does not threaten the propulsion system. 
Considerations of market capacity and minimum mass return for financial viability indicate that the required production of volatiles is in the order of 5000 tonnes in 6 months. This gives a required extraction rate of about 1.2 tonne per hour.

If it is assumed the product mass is $100 \%$ water, and apply latent heats of fusion and vaporization, we find the required heating power is about $1 \mathrm{MW}$, to melt and vaporize this quantity. Note that this is only half or less of the energy input required in extraction of volatiles from carbonaceous chondrite -type asteroidal regolith processing.

If there is no need to vaporize the recovered liquid, then the heat input power requirement is lower again, by a further factor of ten. This is the minimum heat power requirement under the simplest version of the "Kuck process".

It is noteworthy that the power needed for the solar thermal rocket is about $750 \mathrm{~kW}$ for long period thrusting (say 6 months).

Thus it appears that the same solar collector of approximately $1 \mathrm{MW}$ will meet the requirements for both processing and propulsion, in the case of the Kuck volatilization process.

What mass throughput and energy requirements do the various process equipment items have?

As noted elsewhere, this requirement may be relaxed if orbit phasing constraints allow a longer stay time, and therefore longer mining season. Another way of buying more time, and hence permitting a lower equipment mass, is to choose a higher energy outbound trajectory which "gets you there earlier" albeit at higher outbound $\Delta \mathrm{v}$ cost.

If the operation consists of drilling and extraction using a hot-gas lixiviant (Kuck's process) then the total drilling and extraction equipment mass could be under 1 tonne 


\subsection{Mass budget and system integration considerations}

Assume a single launch, with no on-orbit assembly. The mass which can be launched outbound to a Near Earth Asteroid is then assumed to be 5 tonnes, this being the maximum payload of a Russian Proton rocket for injection into a Mars or Venus transfer orbit: these missions have $\Delta v$ 's of approx $5 \mathrm{~km} / \mathrm{sec}$, similar to the mission being envisaged.

The quoted best power to mass ratio of solar photovoltaic arrays is now somewhat below $10 \mathrm{~kg} / \mathrm{kW}$. Thus a $200 \mathrm{~kW}$ array will mass $2,000 \mathrm{~kg}$. An inflatable solar collector will have a much lower mass than this (say 50 to $100 \mathrm{~kg}$ ). The recent Shuttle-launched INSTEP inflatable dish collector had areal density of $0.4 \mathrm{~kg} / \mathrm{m}^{2}$ and diameter of 14 metres.

Instruments for navigation, surveillance, remote analysis, sample analysis, etc., may require $50 \mathrm{~kg}$.

The materials storage bag has to hold 4,000 tonnes at a density of say 0.2 tonnes/cubic metre. This will require a volume of $25,000 \mathrm{cu} . \mathrm{m}$., and hence a surface area of (say) 6,000 sq.m.; if the areal density of storage bag material is $0.25 \mathrm{~kg} / \mathrm{sq} . \mathrm{m}$., then the bag mass is $1600 \mathrm{~kg}$.(eg., "Monarflex" reinforced polyethylene tarpaulin material, strength 7.3 $\mathrm{kN} / \mathrm{m}$ ). Ordinary industrial "Bulker Bags" have areal density of $\approx 0.5 \mathrm{~kg} / \mathrm{m}^{2}$.

Structure, anchor mechanism, sunshield, etc., can be estimated at $? 300 \mathrm{~kg}$.

This leaves for reclaim and separation equipment a mass budget of possibly $3000 \mathrm{~kg}$, to make up to a total mass of $5000 \mathrm{~kg}$, which is the mass budget of the Proton rocket, into various NEA transfer orbits; in fact however there is some "slack" in the system: $\Delta v$ demand on the Proton can be reduced substantially by lunar flyby on the outbound trip which can give a free $1 \mathrm{~km} / \mathrm{s}$ boost, and hence allow heavier load. 
Before any further detailed consideration of materials reclaim and processing systems and their probable mass, it should be noted that there are two approaches that can be taken:

(i) the material can be processed and separated, and tailings discarded, at the asteroid prior to departure for return to Earth; or

(ii) the raw material can be loaded undifferentiated, then processed during the return flight to produce the required propellant.

The latter choice suggests a larger return departure mass, a lower mass throughput requirement on the processing plant, but demands a certainty that the required propellant is in fact recoverable from the amount of material collected; the latter also contains some mass-saving and time-saving process integration possibilities. Because of the fact that volatiles yield will be unknown until processing takes place, approach (i) will be adopted, at least in this present discussion.

\subsection{Telepresence, telerobotics, and autonomous mining robots}

The remote miner will need a high level of autonomy because it will be several to many light-minutes away from active human control: real-time remote control will simply not be responsive enough. The miner will have to be imbued with at least a level of intelligence that looks after housekeeping tasks, and makes the system go into a safe shutdown in event of anything unexpected. Preferably it should contain a level of intelligence capable of accepting, then unfolding, expanding, and interpreting, then implementing, high-level instructions.(see Pidgeon et al., JBIS $\underline{45}$, pp87-92)

Reviews of Artificial Intelligence literature suggest that there are two alternative approaches to developing "empirical" AI for autonomous vehicles: one is to "train" a neural net to experimentally attain the required goal; the other is to develop a rule based expert system and then implement it using fuzzy logic.

Telepresence and teleoperation have other problems apart from time-of-flight signal delay: they also require very wide bandwidth, high data rate communications links 
between the machine and the operator. Nevertheless, mines in Sweden and in Canada operate multiple drilling jumbos (Kiruna, Sweden) and trucks and load-haul-dumps (Inco, Eagle Point, Canada) from remote locations. A Robbins raise drill was used at the Hilton mine, near Mt. Isa, to bore an internal vent shaft, and was operated totally by remote control from a Robbins control room in Seattle, using the public telephone system. Apparently, remote controlled / automated LHDs have also been trialled at Isa/Hilton.

There is very rapid development occurring in the field of artificial intelligence, and futher review of this area is beyond the scope of this thesis.

\subsection{Conclusions:}

Conceptual flowsheet development and equipment sizing can be done; initial study suggests that equipment for extraction of 5000 tonnes of volatiles from 10 times that mass of asteroidal regolith in 6 months can be designed to meet a notional mass budget of well under 5 tonnes. 


\section{Chapter 7: Engineering choices: Propulsion and Power}

\subsection{Considerations in propulsion system choice}

The basic parameters which are used to describe the performance capability of rocket engines are exhaust velocity, $V_{e}$, which is self-explanatory, and reported in kilometres per second, or specific impulse, $I_{\text {sp. }}$. Specific impulse is generally expressed in seconds, and represents the number of seconds for which one kilogram of propellant can provide one kilogram force of thrust. (This description of the meaning of specific impulse is not correct in a pedantic sense, and the correct measure is Newton-seconds of impulse per kilogram of propellant, which gives a number ten times larger than the number in seconds, because one kilogram force is approx ten Newtons; nevertheless, seconds continues to be used as the unit for Specific Impulse.) Obviously, the higher $I_{s p}$ is, the more efficient is the propellant useage, and the less is required to achieve any given $\Delta v$. $I_{s p}$ in seconds is numerically equal to exhaust velocity $V_{e}$ in $\mathrm{km} / \mathrm{sec}$ multiplied by 100 .

As discussed earlier, it is usual to characterize the energy demand of a space mission by the velocity change, $\Delta v$, required to accomplish it.

The "Rocket Equation" is a rewrite of Newton's Second Law of Motion, and relates velocity change $\Delta v$, exhaust velocity $V_{e}$, mass of rocket at departure $M_{0}$, and mass of propellant $\mathbf{M}_{\mathbf{p}}$ :

$$
\Delta v=V_{e} \ln \left(M_{o} /\left(M_{0}-M_{p}\right)\right)
$$

Thus a high final velocity is achieved by a high propellant mass fraction, and/or by a high exhaust velocity.

The choices available depend on whether the velocity change must be delivered rapidly, i.e. high acceleration required, or whether it can be delivered slowly over a long period. If the former then a high thrust rocket motor is needed, and the choices are limited to chemical rockets or nuclear thermal rockets. Even then, the mass that would be able to 
be returned to LEO would be limited, dependent on the rocket thrust and burn time available, and the minimum required acceleration. If low acceleration, non impulsive propulsion is allowable, then more possibilities exist.

\subsubsection{Impulsive (high thrust) systems}

Chemical rockets may either burn propellants that have been carried up from the earth's surface or propellants that have been produced from the mined resource.

Space-storable chemical rockets to date have almost invariably used as propellants, monomethyl hydrazine and nitrogen tetroxide; this combination is readily storable for years, and has an $I_{s p}$ of 270 to 300 seconds. Alternatively, solid rockets having an $I_{s p}$ of $250 \mathrm{sec}$. have been used.

The formula that determines the $I_{\mathrm{sp}}$ of a thermally generated jet is

$$
\mathrm{I}_{\mathrm{sp}}=270(\mathrm{~T} / \mathrm{MW})^{0.5}
$$

where $\mathrm{T}$ is temperature of exhaust gases in degrees Kelvin, and MW is their average molecular weight.

However, because the whole point of asteroid mining is to deliver large amounts of mass to LEO at less cost than launching it from Earth, the use of Earth-sourced fuels is not helpful. If the resource contains easily recoverable volatiles then it is possible that oxygen and fuel can be chemically extracted, stored, and then burned, rapidly, to provide high thrust and acceleration. The thrust levels possible might be very large, e.g. equal to the Space Shuttle Main Engines, which give 2,000,000 Newtons each, in vacuum. Storage of $\mathrm{LOX}$ and $\mathrm{LH}_{2}$, however would require cryogenic refrigeration and insulation, a major mass burden. 
Nuclear rockets were being actively developed 20 years ago (the NERVA project). The concept was to pump hydrogen through the core of a nuclear reactor to heat it to exhaust as reaction fluid.

A full scale operating nuclear rocket was constructed and ran, and achieved an $I_{s p}$ of 800 seconds, and nearly attained flight readiness before funding was withdrawn (Dewar,1994). Nuclear rockets are now perceived by many to present unacceptable risk of release either of fission products from possible rupture of fuel rods during operation within the atmosphere, or of fuel, which would be highly enriched uranium or plutonium, in the case of a crash or of the necessity to destroy the vehicle during ascent into orbit for range safety reasons. Thus it is likely that nuclear rockets will only be accepted if they are launched 'cold' and are started from "nuclear - safe" orbit, generally taken to be several hundred kilometres altitude. However, the concept has been fully engineered, with major recent technology recovery by Grumman focussing on particle bed reactors ( $R$ Haslett, 1993). The advantage of the nuclear thermal rocket is that the energy source - high enriched uranium - is of very high energy density, and the reactor can use any non coking volatile material as its coolant/working fluid/propellant.

There are other nuclear rocket concepts, which are potentially very powerful, capable of very large impulses (mass times velocity): Project Orion, which proposed propulsion by rapid repeated nuclear explosions behind a pusher plate; and Zubrin's "Nuclear Salt Water Rocket" (Zubrin, 1991), which proposes fissioning and expulsion as superheated vapour of an aqueous solution of enriched uranium salt. Another nuclear pulse - driven concept is the Medusa, described in Solem (1993). The exhaust in these cases would be highly radioactive, so the rocket could not be used anywhere except in space, for orbit transfers, but they would provide truly staggering performance, and make sciece-fictionlike missions easy! ( $I_{\text {sp }}$ of $10,000 \mathrm{sec}$ and thrust of many kilonewtons...)

Speculative, but approaching technical feasibility, are fusion power and propulsion systems, using either magnetic or inertial electrostatic confinement (e.g. Shultz, 1994). 


\subsubsection{Non impulsive (low thrust) systems}

Low thrust systems are incapable of lift off from a planetary surface, because of their poor thrust to mass ratio, but may still be able to provide large $\Delta v$ performance, because of very long thrusting times. The acceleration provided by low thrust systems is necessarily very small, but its continued application over many days or weeks can still give a large $\Delta \mathrm{v}$.

Non impulsive propulsion systems generally use an inert propulsion mass (reaction mass) and an external (generally electrical but possibly solar) power supply. Examples are:

- $\quad$ Resistojet: Nitrogen gas expelled after heating by electrical resistors; reject due to very poor specific impulse: $I_{s p}=170 \mathrm{sec}$. Electrically boosted monopropellant hydrazine, $I_{s p} \approx 600 \mathrm{sec}$, depending on exhaust temperature.

- Ion rocket: Mercury, Argon or Xenon ions accelerated by electrostatic repulsion; excellent specific impulse: $I_{\mathrm{sp}}=2000$ to $6000 \mathrm{sec}$; technology is well advanced, but thrust levels are very low: 0.1 to 0.3 Newton. (Fearn, 1982; Rudolph \& King, 1984)

- Arcjet: Ammonia gas heated by electric arc; models have been run for several weeks continuously, at tens of kilowatts power levels and several Newtons thrust levels; specific impulse is about twice that of chemical rockets, i.e., 600 to $800 \mathrm{sec}$, and they can potentially use any gas as propellant; technology well advanced. (Nakanishi, 1985; Deininger \& Vondra, 1991)

- Mass driver: Electromechanical accelerator; this propulsion method would provide thrust by ejecting at high velocity any waste solids, e.g. silicate "tailings" from prior or concurrent materials processing; thrust can be quite high; technology was under development for a few years only at a couple of institutions, MIT and Princeton. (O’Neill \& Snow, 1979; Kolm et al, 1979) 
The electrical power requirements of the above propulsion methods range from 5 to $10 \mathrm{~kW} / \mathrm{N}$ for the low $\mathrm{I}_{\mathrm{sp}}$ Mass Driver technology, to intermediate power requirements for the arcjet, up to 20 to $50 \mathrm{~kW} / \mathrm{N}$ for Ion rocket technology.

- Solar thermal rocket; this method would use solar heat to pressurise and expel any volatile material; technology immature. $I_{\text {sp }}$ could be anywhere from 200 to 800 or more seconds, depending on the working fluid's temperature and molecular weight (Shoji, 1985). See also "Inflatable Concentrators for Solar Propulsion and Dynamic Space Power", Grossman and Williams, 1990. (reports the work done at L'Garde Inc, Tustin, Calif).

- Solar sails: these obtain their reaction force from the reflection of sunlight, thus use no mass at all, and require no power supply, however the thrust is very low.

- Combustion of electrolytically produced fuel: as a possibility, hydrogen and oxygen could be derived from asteroidal water ice, using solar or nuclear generated electricity, and burned in a low thrust long running engine.

\subsection{Considerations of Power Sources}

Most of these propulsion methods require a supply of electrical power. A power source is also needed for the mining and processing operations. This can be from solar photovoltaic panels, a solar concentrator mirror driving a heat engine and thence an electrical generator, or from a nuclear reactor or radioisotope generator. Speculatively, fusion power should also be considered, both as a power source and applied directly for thrust.(refs).

The largest near term space electrical power systems are Photovoltaic (PV) or solar thermodynamic and in the range 50 to $100 \mathrm{~kW}$, but there is no technical reason to limit their growth.

Comparison between PV and solar thermal power must note the following: 
- the form of energy required for volatiles recovery, and for Mond process, is primarily thermal.

- solar thermal rocket technology has been developed (by Shoji et al) to the point where it is a reasonable option

- the thermal power requirements for processing and for propulsion for the examples considered in this thesis, are similar enough to suggest the use of the same collector for both purposes, giving major mass savings

- ultra lightweight inflatable solar collectors have been developed by L'Garde Inc..

- $\quad$ PV technology has also been getting lighter but suffers the disadvantage of at best $20 \%$ conversion efficiency.

- $\quad$ electrical energy has the advantages that it is needed, anyhow, for electronics and electromechanical purposes

New lightweight solar arrays using amorphous silica on flexible substrates are quoted to have power to weight ratio above $100 \mathrm{~W} / \mathrm{kg}$. (Stuart \& Gleave, 1990)

The SP-100 nuclear reactor is quoted to have a power to mass ratio of $25 \mathrm{~W} / \mathrm{kg}$ (Kelley, Boain \& Yen).

From the above considerations, the following Propulsion - Power Matrix can be drawn:

Table 7.1 Propulsion - Power Options

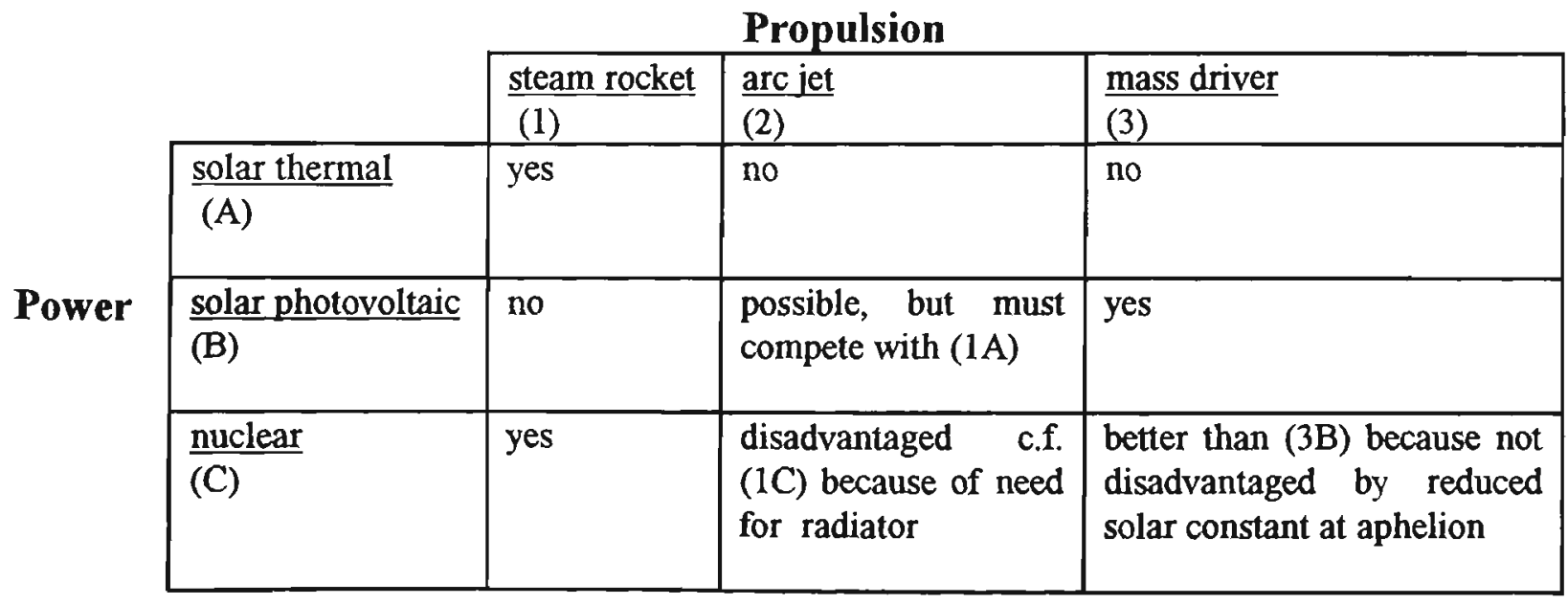


Given that "bootstrapping" - i.e., the use of in-situ derived propellants for return propulsion - is the major project enabler, the propulsion system choices reduce to the following options:

(i) mass driver powered by PV or nuclear reactor and using regolith silicate;

(ii) arcjet powered by PV or nuclear reactor and using any volatile (preferably steam because of its availability);

(iii) thermal rocket using any volatile (preferably steam) and powered by solar or nuclear heat.

System choices (2B) and (2C) above require demonstration that arcjets can work acceptably with steam. System choice $1 \mathrm{~A}$ is that proposed by $\mathrm{J} \mathrm{S}$ Lewis, and followed most fully in this thesis; system choice $1 \mathrm{C}$ is the preferred option of Zuppero et al, in their discussions of comet mining, and in particular, the exploitation of 1979 WilsonHarrington. System choice 3C was the preferred option of O'Leary and of O'Neill in their writings of the early 1980's and late 1970's.

A notable advantage of the mass driver is that it can use the residue from the volatiles and metals extraction process; thus reducing the total mass to be mined; and that the processing can take place during the course of the return trajectory; thus reducing overall mission time and improving financial feasibility.

Simplicity and light weight suggest use of arcjet with nuclear power, or a solar thermal steam rocket. The photovoltaic powered arcjet is also possible, but is disadvantaged by its higher mass / power ratio; although the PV array pointing requirement is much less stringent than is the pointing requirement for a solar concentrator. The arcjet also has in its favour a much higher probable specific impulse, $I_{\text {sp }}$.

\subsection{Conclusions:}

The "best" system choices for power and propulsion are interrelated, and depend also on the desired product and processing options and requirements. The simplest near-term choice is solar thermal power and propulsion, using water ice as reaction mass. 


\section{Chapter 8: Project Selection Criteria}

\subsection{Economic Analyses}

There have been various papers addressing the economics of mining the asteroids and mining the moons of Mars; (e.g., Leonard, Blacic \& Vaniman, 1987; Cordell \& Wagner, 1986).

Several papers discuss "Figures of Merit" for assessment of missions and use mass payback ratio (MPBR) as a "Figure of Merit" for comparison of alternative asteroid mining missions (e.g., Lewis, 1993; Lewis, 1991; Meinel \& Parks, 1985).

\subsubsection{Findings from the Literature}

Several papers note the important fact that time-cost-of-money puts an upper limit on the allowable project cycle time, and that time from capital committment to initial income from product sales is critical. Meinel \& Parks, 1985, suggest that it is necessary to achieve an internal rate of return (IRR) in excess of 30\% per annum!! However, Collins (pers. comm., 1996), points out that the Japanese time-cost-of-money allows a much lower venture capital I.R.R.)

Some other observations from these and other papers are as follows:

"We need some way of quantitatively assessing the merit of a very large number of (competing) combinations of minesites, ores, processes, products, and destinations..... (There is) the very important task of identifying and evaluating the "big picture". How (can one make) these various technologies mesh together to give the best overall system? Here the important concept of Figure of Merit has been evolved to enable a quick quantitative evaluation of the overall mission impact of various candidate technologies." - (Lewis, Ramohalli, \& Triffet, 1990 - this author's italics). 
Leonard, Blacic, and Vaniman used the US National Commission on Space report, "Pioneering the Space Frontier" to define their minimum market size and rate of development. Assumed volatiles, ie water, to be the essential product. They noted that time-cost-of-money requires the project developer to minimize startup capital; also the equipment must arrive on 'minesite' ready-to-start with no delay (this implies minimization of on-site infrastructure construction requirement). They assumed 200 tonnes of mining equipment, nuclear power, manned, ion rocket technology. They suggested "Phobos manufacturing capability may develop before manufacturing on the Moon."

They concluded: "Using propellent (for nuclear-ion rocket propulsion) lifted from Earth, the breakeven point (for feasibility) is at an Earth-to-leo launch cost (not below) between $\$ 500$ and $\$ 1000$ per kilogram for volatiles from the Moons of Mars to be profitable. If propellent for the ion thruster can be derived from extraterrestrial resources, the breakeven point moves much closer to the very optimistic figure of $\$ 200$ per kilogram Earth launch cost."

Lewis (1991a) provided several "mission architectures" for delivery of water from NearEarth asteroids to HEEO, and calculated MPBR for these missions, for single and multiple return trips.

Ramohalli, Kirsch and Priess have shown that 'Figures of Merit' may be useful for purposes of inital screening of a myriad of concepts. They looked particularly at Mars Sample Return missions, and used FOM approach to assess competitiveness of ISPP concepts. FOM considers specific impulse, mass ratio, reliability, inverse risk, repairability, ease of autonomous controls, scalability and adaptability. They gave several possible definitions of FOM;

1 mass payload/mass launch

2 standard cost/total lifecycle cost of the mission

3 mass sample returned to leo/mass craft launched from leo

4 total useful mass/mass at launch

5 any of above, modified by factors for reliability, repairability and inverse risk. 
Using Lotus 1-2-3 spreadsheet they experimented to find optimum FOMs given multiple independent variables. They noted that the high Isp of $\mathrm{LH}_{2}-\mathrm{LOX}$ is not in itself a good enough reason to choose this propellant over other alternatives.

In “An Investment Analysis Model for Space Mining Ventures" (1991), Kurt Oxnevad found that:

(i) "Through extensive sensitivity analysis, it was... shown that launch cost (was) not a critical parameter."

(ii) Traditional MPBR "does not take into account development costs, difference in value between mass launched and mass returned, nor does it take into account the time-cost of money." Oxnevad went on to point out that rigorous economic comparative analyses should emphasise NPV rather than MPBR.

Oxnevad also discussed the "International Asteroid Mission", a study project carried out by students of the International Space University in 1990. This plan assumed a manned mission, and was based on sale of mass to a Low Lunar Orbit space station site. Oxnevard, as a member of the study group, suggested $20-25 \%$ nominal discount rate (used for comparable high risk terrestrial projects), and calculated its NPV as negative $\$ 60$ billion! Clearly, high capital cost, high discount rate, and long payback time all contributed to this 'show-stopper' finding.

Cutler \& Hughes, 1985, made similar points:

(i) "high MPBR is not particularly important. Low initial capital is important.... Optimizing selected physical parameters such as delta-v or Isp does not in general lead to most economical system." 
(ii) "A general economic methodology to evaluate schemes for extraterrestial resource utilization is needed. At the moment no standardized method exists for researchers to compare their schemes on a common basis. They are not able to evaluate the effects of specific innovations. Each prior study calculated costs differently and set up a different manufacturing scenario without isolating the economic effects of each system component. Thus, quantitative comparison between these studies is not possible."

There is discussion as to whether comet mining, in particular, would be better done via aphelion mining, with the concomitant burdens of long project time, impacting on NPV; low solar flux, impacting on power supply; and long signal time-of-flight delay, impacting on ease of teleoperation; or perihelion mining, with the problem of very large delta-v for out and return flights, impacting on available throw-weight outbound; and on proportion of payload that must be expended as propellant on return trip; and very short mining season duration, impacting on the total mass that can be mined and processed in the time available. These choices demand economic, as well as technical, analysis.

To summarize, there is an apparent need, identified by several workers, for a robust general approach to comparing hypothesised space mining projects; and for performing realistic feasibility assessments. This chapter addresses these requirements.

\subsubsection{Simple financial feasibility example}

Any mission plan must meet constraints based on required rate of return, mission cycle time (which would itself depend on the orbital synodic period for return visits to the target), the mass that can be launched as a remote/autonomous miner to the target, and the propulsion choice and $\Delta v$ for the return journey. And of course, the value per kilogram of returned product.

Let us assume that a project is envisaged to return 2,000 tonnes of useful material to Low Earth Orbit, and that it is worth, in orbit, $\$ 500$ per kilogram. (Note that present freight cost to LEO is approx $\$ 10,000$ per kilogram). Thus the value of returned 
product is $\$ 1$ billion. Let us also assume that the reaction mass can be extracted from the mined material, and that it will be used in a low thrust long operating propulsion system, either an arcjet using the permanent gases or the volatiles, or an electromagnetic mass driver using silicate fines, or a solar thermal rocket using recovered volatiles. Flight time is long, and it is determined by the available power. Long flight time impacts on project financial viability, so duration of transfer trajectories out and back, available power for processing and for propulsion, and the power requirements of the propulsion system become critical considerations.

Assuming $10 \mathrm{~kW} / \mathrm{N}$ arcjet power to thrust ratio and a power supply of $200 \mathrm{~kW}$ : for a return $\Delta v$ of $1 \mathrm{~km} / \mathrm{sec}$, simple use of $F=m \times a$ shows that time required will be about 1,000 days. Outbound travel time for the miner might reasonably be about 400 days.

The total project time, therefore, might be about 6 years from commitment. Net Present Value (NPV) of $\$ 1$ billion at 6 years in the future at $30 \%$ interest rate is about $\$ 210$ million, so the budget for such a project certainly could not go much over $\$ 150$ million, in view of the high technological risk.

The rest of this Chapter is intended to derive more rigorously a generic approach to performing a Feasibility Study for a hypothetical asteroid mining project.

\subsection{Technical and economic interrelations - the "Spider Diagram"}

As is now clear from earlier discussion, much study is needed to define realistic project alternatives, including :

- $\quad$ target asteroids / comets

- $\quad$ propulsion methods and propellants

- $\quad$ power sources

- $\quad$ materials to be reclaimed

- materials reclaim and processing methods

- guidance, navigation, and control, both outbound and return 
- $\quad$ autonomous control of mining and processing activities

- $\quad$ sizing of minimum feasible project, and

- financial considerations

The following linkages are apparent:

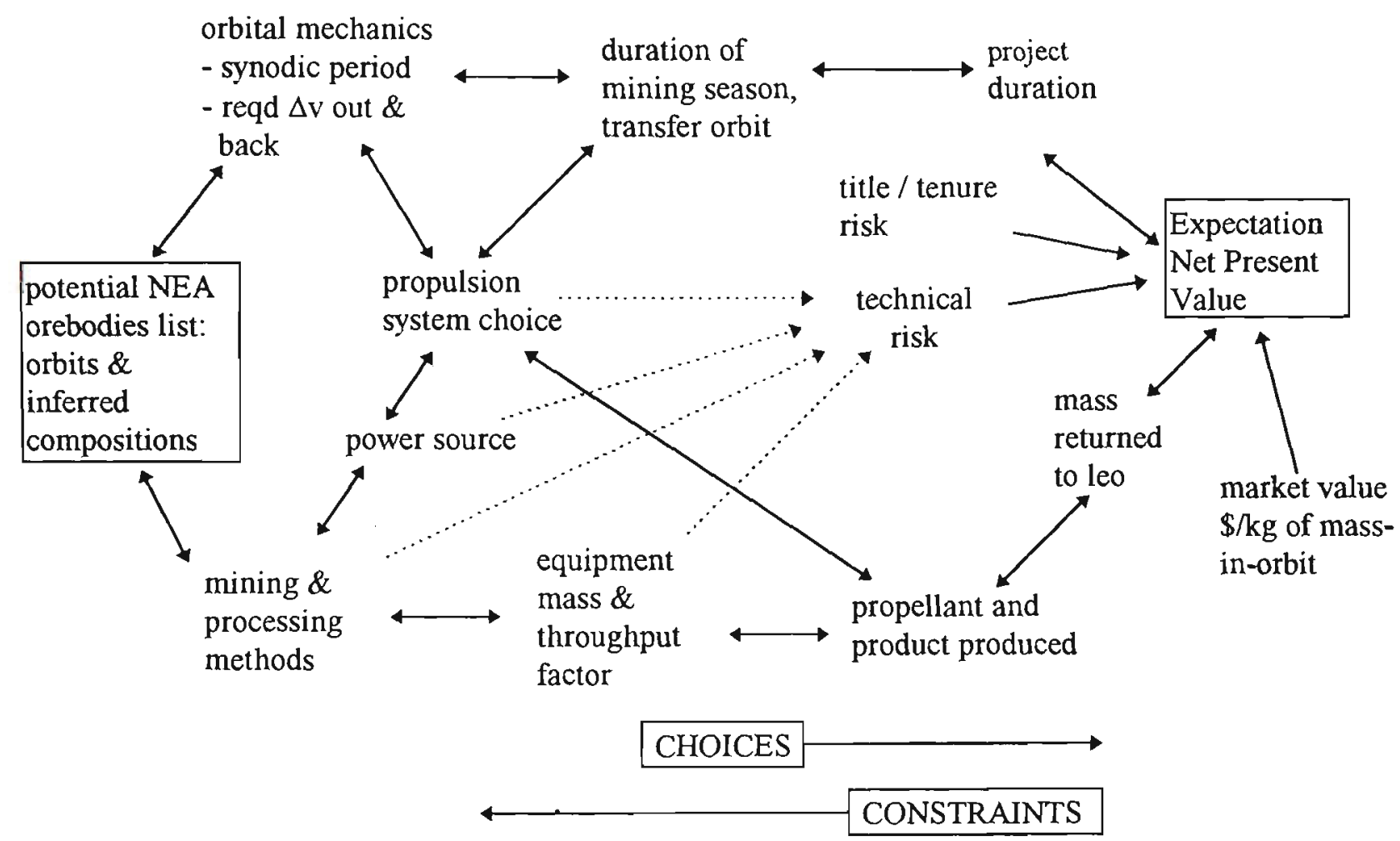

Figure 8.1 Project Feasibility "Spider Diagram"

These choices are interrelated, as selection of a particular option in one area introduces constraints in the other areas. Also, different levels of knowledge and technical maturity apply to the various options.

Mechanisms for ensuring political acceptance of a right to mine the resource are considered in Appendix 1. 


\subsection{NPV Discussion and formula derivation}

Economic "figures of merit" used to assess financial feasibility of proposed projects are: Payback Period ( = Net Investment / Net Yearly Cash Benefit ) for quick analysis, and: Net Present Value, as a more accurate measure of project merit over a project time period of (say) up to 10 years.

Internal Rate of Return (IRR) is the discount rate at which NPV equals zero, i.e., is the implied interest rate that the project pays its owners.

Mining companies (and more generally, banks and other large investors) regard a project that can pay back its capital in 3 or 4 years as attractive, and one which will take 6 or more years as unattractive and not worth investing in. Considerations of attaining strategic political or market positioning may however override these rules of thumb.

NPV calculates the present value of receipts of money to be received " $n$ " years in the future, taking into account the foregone interest that the invested money could have been earning. The longer you have to wait for the income, the more heavily discounted it must be, in the NPV calculation.

The following rules generally apply:

- capital - minimize

- $\quad$ requirement for technical innovations - minimize

- time before income stream - minimize

- technical risk - minimize

- $\quad$ revenue - maximize

- $\quad$ multiple products - desireable

- $\quad$ sensitivity to market fluctuations - minimize

- $\quad$ multiple potential customers - maximize

- $\quad$ large market - desireable 
It is important to try to find a way to compare the financial feasibility of competing space mining mission proposals, such as:

- volatiles from comet core (aphelion mission)

- volatiles from comet core (perihelion mission)

- volatiles from C-type asteroid (aphelion mission)

- $\quad$ metals and volatiles from C-type asteroid

- metals from M-type asteroid

- $\quad$ PGMs only from C-type asteroid

- $\mathrm{LOX}, \mathrm{LH}_{2}$ from lunar polar ice

- bootstrapping vs non-bootstrapping missions to NEAs

- non-bootstrapped raw mass return from an Arjuna

- volatiles from Phobos or Deimos

In order to carry out these comparisons, it is necessary to rewrite the formula for Net Present Value in terms of astrodynamic and the Rocket Equation variables.

\subsubsection{Breakeven Analysis}

Breakeven occurs when fixed plus variable costs equate to revenue. In space mining missions, it seems likely that the great majority of costs will be fixed costs, namely, equipment development and acquisition, launch and control. These are in the nature of sunk costs and are not proportional to output. The implication of this for asteroid mining is that once the initial payload is returned and sold, very large reductions in the price of product can be sustained, limited only by the need for reinvestment capital. 


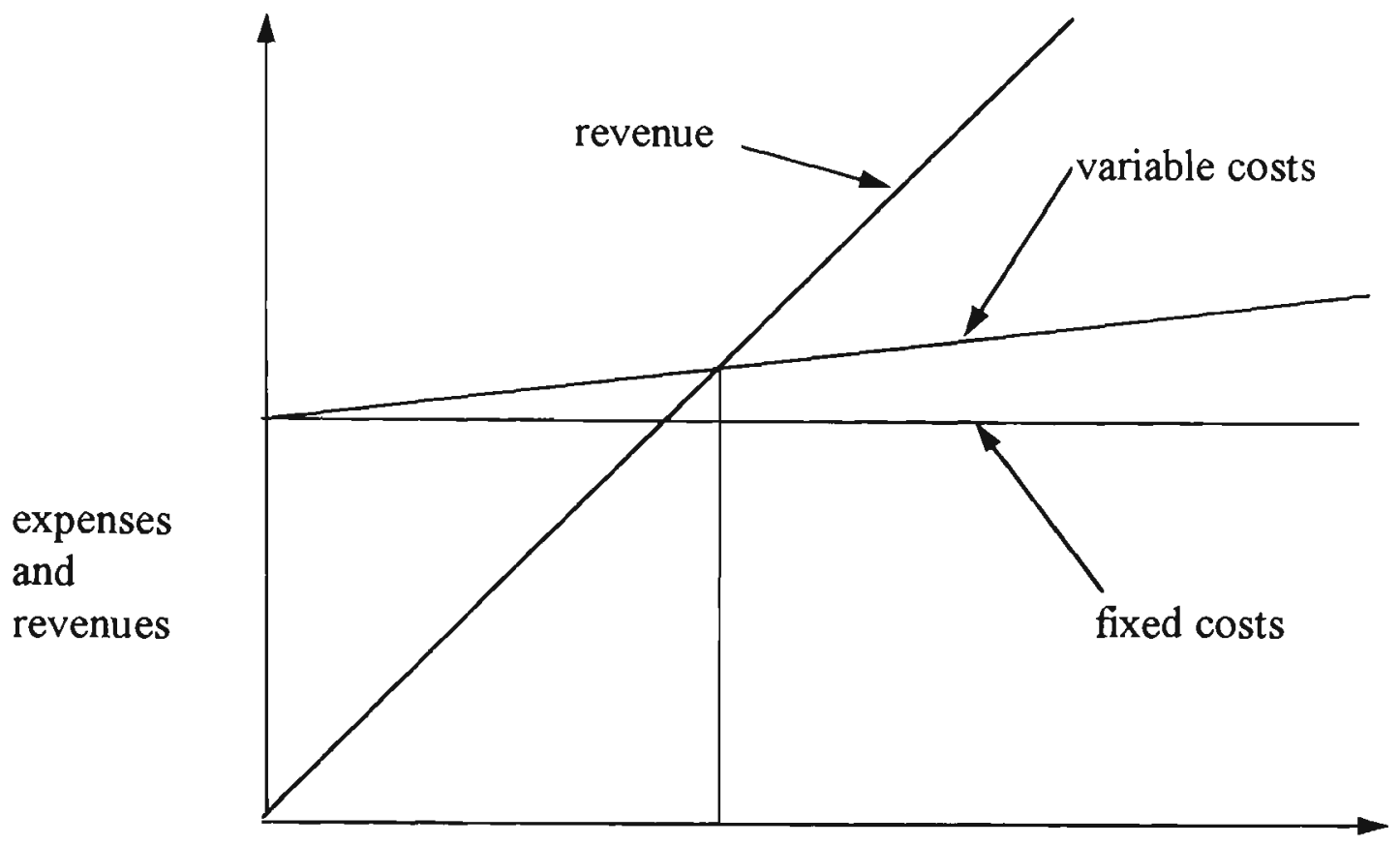

sales from production

Figure 8.2 Breakeven Analysis diagram

In addition, it is necessary to incorporate both a classical Sensitivity Analysis, and a Reliability or Probability-of-Success Analysis.

\subsubsection{Sensitivity Analysis}

Table 8.1 Sensitivity Table

\begin{tabular}{|c|c|c|c|c|c|}
\hline \multirow[t]{2}{*}{ cost of capital } & & \multicolumn{4}{|l|}{$(30 \%)$} \\
\hline & \multicolumn{5}{|l|}{$(25 \%)$} \\
\hline \multirow[t]{2}{*}{$(20 \%)$} & & \multicolumn{4}{|c|}{ Market Size (tonnes per mission) } \\
\hline & & 1000 & 2000 & 5000 & 10000 \\
\hline Competing & 100 & $1 \times 10^{8}$ & $2 \times 10^{8}$ & $5 \times 10^{8}$ & $1 \times 10^{9}$ \\
\hline Launch & 200 & $2 \times 10^{8}$ & $4 \times 10^{8}$ & $1 \times 10^{9}$ & $2 \times 10^{9}$ \\
\hline Costs & 500 & $5 \times 10^{8}$ & $1 \times 10^{9}$ & $2.5 \times 10^{9}$ & $5 \times 10^{9}$ \\
\hline \multirow[t]{2}{*}{$\$ / \mathrm{kg}$} & 1000 & $1 \times 10^{9}$ & $2 \times 10^{9}$ & $5 \times 10^{9}$ & $10 \times 10^{9}$ \\
\hline & 2000 & $2 \times 10^{9}$ & $4 \times 10^{9}$ & $10 \times 10^{9}$ & $2 \times 10^{10}$ \\
\hline
\end{tabular}


A Sensitivity Analysis is intended to answer the questions:

- what happens if costs to LEO drop to (say) $\$ 500 / \mathrm{kg}$ ? (or $\$ 200 / \mathrm{kg}$, vide P Collins)

- what if lunar LOX, $\mathrm{LH}_{2}$ are deliverable to LEO at (say) $\$ 500 / \mathrm{kg}$ ? (JS Lewis would strongly discount this as a possibility).

- what if market size is only 500 tonnes per year in LEO?

- $\quad$ if we increase output by 50 or $100 \%$ can we still sell it?

\subsubsection{Reliability / Probability Analysis}

An appropriate Figure of Merit for a risky commercial enterprise is "expectation value of NPV", where the expectation value of NPV, its most likely value, weighted by probability of outcome, is:

$(\mathrm{NPV})_{\mathrm{w}}=\mathrm{NPV}_{1} \cdot \mathrm{p}_{1}+\mathrm{NPV}_{2} \cdot \mathrm{p}_{2}+\mathrm{NPV}_{3} \cdot \mathrm{p}_{3}+\ldots$.

where probabilities $p_{1}, p_{2}, p_{3}, \ldots$ etc add to $=1$.

\subsubsection{Net Present Value Derivation \&Calculation Process}

Present Value of a Receipt $R$ obtained in year $n$ is:

$P V=R .(1+i)^{-n} \quad$ where $i$ is the interest rate paid for risky investment capital.

Table 8.2 shows PV's for space-sourced mass (at $25 \%$ pa and $\$ 1000 / \mathrm{kg}$ value of mass in orbit).

Table 8.2 Present Value versus Time to Payment

\begin{tabular}{|l|l|l|l|l|l|l|l|}
\hline Time (years) & 0 & 1 & 2 & 3 & 4 & 5 & 6 \\
\hline & & & & \$million & & & \\
\cline { 2 - 8 } & 500 & 400 & 320 & 255 & 205 & 165 & 130 \\
tonnes \\
returned
\end{tabular}


$\mathrm{NPV}=\sum_{\mathrm{i}=1 . \mathrm{n}} \mathrm{R}_{\mathrm{i}} \cdot(1+\mathrm{i})^{-\mathrm{n}}-\mathrm{C} \quad(\mathrm{C}$ is invested Capital)

NPV in the comet or asteroid mining case depends on:

cost to launch and conduct the mission

mass returned and what you can sell it for

time it takes to accomplish

Outbound mass consists of

final stage propellant to give reqd $\left(\mathrm{v}_{\infty}+\Delta \mathrm{v}_{\mathrm{DS}}\right)$

mining and processing equipment

solar collector

payload bag

Whilst outbound $\Delta \mathrm{v}$ is not critical, except within the constraints of the launcher capability, return $\Delta \mathrm{v}$ must be minimized; and duration of mining season should be maximized, consistent with minimizing total mission time and maximizing mass returned.

The implications for asteroidal or cometary resource return projects are that missions taking longer than (say) three years would have to have very good MPBRs (mass payback ratios), in order for the NPV to be positive.

For the "Apollo-type" asteroid or comet mining case, with a single payload return, the formula for NPV can be expanded as follows:

For a single payback receipt, $\quad \mathrm{NPV}=\mathrm{R}(1+\mathrm{i})^{-\mathrm{n}}-\mathrm{C}$

(i) $\mathrm{n}=$ time from capital-raising to launch $+\mathrm{T}_{\text {transfer orbit }}+$ time from Earth-capture to sale

(ii) $\mathrm{T}_{\text {transfer orbit }}=\left(\right.$ semi-major axis, "a") ${ }^{3 / 2}$ (years) 
(iii) Receipt, $\mathrm{R}=\$ / \mathrm{kg}$ value of mass in orbit $\times$ mass of volatiles returned for sale;

- note that $\$ / \mathrm{kg}$ launch cost sets upper limit on what the resources enterprise can charge for mass in orbit.

(iv) $M_{\text {returned }}=M_{\text {produced }} \times e^{-\Delta v / v_{e}}$

- from the Rocket Equation, which says $\Delta v=v_{e} \times \ln \left(M_{\text {start }} / M_{\text {finish }}\right)$.

- $\quad$ (this neglects equipment mass, small compared with the returned payload)

(v) $\mathrm{M}_{\text {produced }}=\mathrm{M}_{\text {mined }} \times \%$ recovered volatiles

(vi) $M_{\text {mined }}=M_{\text {mpe }} \times$ throughput factor " $\mathrm{f}$ " $\times \mathrm{t}_{\text {mining stay }} ; \mathrm{M}_{\text {mpe }}$ is the mass of the mining and processing equipment; and " $\mathrm{f}$ " is $\mathrm{kg} /$ day handled per kilogram of equipment, and is considered likely to be approx 200 , based on discussion in Chapter 6.

(vii) $v_{e}=270(\mathrm{~T} / \mathrm{MW})^{0.5}$ metres/second, where $\mathrm{T}$ is in Kelvin and $\mathrm{MW}$ is molecular weight of the exhaust gases. (Note that you divide the exhaust velocity by 10 to get $\mathrm{I}_{\mathrm{sp}}$ in seconds.)

(viii) $\Delta v_{\text {retum }}=\sqrt{\Delta v_{\text {ecliptic-transfer }}^{2}+\Delta v_{\text {inclin-change }}^{2}}$--if the line of nodes is coincident with the line of apsides

(ix) $\Delta v_{\text {incl }}=(0.5 \times \mathrm{i}($ degrees $)) \mathrm{km} / \mathrm{s}$

(x) Mass launched $=M_{\text {mpe }}+M_{\text {outbound fuel }}+M_{\text {power source }}+M_{\text {payload bag }}+M_{\text {instr \& control }}$ $(=$ mass of mining and processing equipt + mass of power supply + mass of cargo container + mass of instrumentation and controls)

(xi) $\mathrm{C}=$ capital costs + running costs until product return

$$
\begin{aligned}
=\left(\left(M_{m p e}+M_{p s}+M_{i \& c}\right) \times(\$ / k g \text { purchase cost }+\$ / k g \text { "airfreight" to orbit })+\right. \\
\quad(\text { annual budget }) \times n \text { yrs })
\end{aligned}
$$




$$
\text { So, } \begin{aligned}
\text { NPV } & =R(1+\mathrm{i})^{-\mathrm{n}}-\mathrm{C} \\
& =\$ / \mathrm{kg}_{\text {orbit }} \times \mathrm{M}_{\text {retumed }}(1+\mathrm{i})^{-(\mathrm{a} 3 / 2)}-\mathrm{C} \\
& =\$ / \mathrm{kg}_{\text {orbit }} \times \mathrm{M}_{\text {prod }} \times e^{-\Delta v / v_{\mathrm{e}}} \times(1+\mathrm{i})^{-(\mathrm{a} 3 / 2)}-\left(\left(\mathrm{M}_{\mathrm{mpe}}+\mathrm{M}_{\mathrm{ps}}+\mathrm{M}_{\mathrm{i} \& \mathrm{c}}\right) \times \$ / \mathrm{kg}+\right. \\
& \text { annual budget } \times \mathrm{n})
\end{aligned}
$$

(xii) Note that $\mathrm{M}_{\mathrm{prod}}=\left(\mathrm{M}_{\mathrm{mpe}} \times \mathrm{f} \times \mathrm{t}\right) \times \%$ recovered volatiles;

then (finally):

$$
\begin{aligned}
& \mathrm{NPV}=\$ / \mathrm{kg} \text { orbit } \times \mathbf{M}_{\text {mpe }} \times \mathbf{f} \times \mathrm{t} \times \% \text { recov } \times \mathrm{e}^{-\Delta \mathrm{v} / \mathrm{ve}} \times(1+\mathrm{i})^{-(\mathrm{a} 3 / 2)} \\
& \left.-\left(M_{m p e}+M_{p s}+M_{i \& c}\right) \times \$ / k g \text { manuf }+ \text { budget } \times \mathbf{n}\right)
\end{aligned}
$$

Note for interest that Mass Payback Ratio is given by

MPBR $=M_{\text {mpe }} \times f \times t \times \%$ recov $\times e^{-\Delta v / v e} /\left(M_{m p e}+M_{p s}+M_{i+c}\right)$

Note that the formulae for $\Delta v$ are given in Chapter 4.

\section{Process for determining NPV}

This applies to mining missions with short stay times centred around aphelion or perihelion, with Hohmann transfers out and back; it assumes that the thrusting time on the return transfer is short c.f. the orbital period of the transfer orbit, i.e., less than (say) 20 degrees of arc. It also assumes that capture into earth orbit is via lunar flyby to remove hyperbolic velocity. The $\Delta \mathrm{v}$ to go from HEEO to LEO has not yet been considered.

The process for determining feasibility is thus as follows:

1. set required payload to be returned.

2. find $\Delta v$ (return) from target body using Hohmann transfer calc or otherwise.

3. adjust for $\Delta \mathrm{v}$ reqd for inclination change (i in degrees): 
4. from $\mathrm{I}_{\mathrm{sp}}$, calculate propellant requirement;

5. determine mining stay time, and assume some recovery (say $10 \%$ of bulk feed); hence determine power reqd by miner to process reqd quantity of volatiles.

6. using same power source, derive "burn time" curve (for interest only), and from it check mass returned.

7. calculate elapsed time from period of transfer orbit.

8. insert all variables into formulae above, and calculate NPV.

\section{Expectation Value of NPV}

Strictly, the NPV calculated above should be discounted to take into account the lessthan-unity chance of success:

$\operatorname{Exp} N P V=p_{1} \times N P V_{1}+p_{2} \times N P V_{2}+p_{3} \times N P V_{3}+$

where $\mathrm{p}_{1}=$ fractional probability of outcome $\mathrm{i}$; e.g., if probability of total success is $80 \%$ and probability of total failure is $20 \%$, then Exp.NPV will be:

Exp.NPV $=0.8 \times \mathrm{NPV}+0.2 \times(-\mathrm{C})$

So finally,

Expectation NPV =

$\mathrm{p}_{\text {success }} \times\left[\$ / \mathrm{kg}\right.$ product in orbit $\times \mathrm{M}_{\text {mpe }} \times \mathrm{f} \times \mathrm{t} \times \%$ recov $\times e^{-\Delta v / m} \times(1+i)^{-a 3 / 2}$

$-\left(M_{\text {launched miner }} \times \$ / \mathrm{kg}\right.$ 'airfreight' $+\left(\mathrm{M}_{\text {mpe }}+\mathrm{M}_{\mathrm{ps}}+\mathrm{M}_{\mathrm{i}+\mathrm{c}}+\mathrm{M}_{\text {cont }}+\mathrm{M}_{\mathrm{prop}}\right) \$ / \mathrm{kg} \mathrm{mfg}+$ annual budget $\left.\left.\times\left(a^{3 / 2}+\mathrm{T}\right)\right)\right]$,

where $\mathrm{T}$ is the total pre-launch project preparation time plus the post-return time to finalise sales.

Then one needs to insert formulae for $\Delta v_{\text {return }}, V_{e}$.

\subsection{Conclusion}

A similar process of calculation can be performed for other mission scenarios as described and discussed in Chapter 5. Examples are developed in Chapter 9. 


\section{Chapter 9 : Project Example Calculations}

In all of the following cases we assume that the desired product for return is water; that the reclaim is to be done by a remote/autonomous miner; that the requirement for simplicity demands that the project rely on a single launch; that the mass budget is restricted to 5 tonnes; and that a solar thermal rocket propulsion system has been chosen, with an operating temperature of $2400 \mathrm{~K}$, a conservative figure for a Rhenium nozzle, and giving an $\mathrm{I}_{\text {sp }}$ of 310 seconds.

Other realistic alternative scenarios could be developed, e.g. assuming PV power and arcjet propulsion, but these have not beèn pursued here, because the intent of this chapter is merely to demonstrate some of the trade-offs which are uncovered.

\subsection{Example \#1: 1989ML ("Apollo-Type") mission}

We wish to deliver 1000 tonnes of volatiles to earth orbit from $1989 \mathrm{ML}$, an Amor. (Assuming that volatiles are indeed obtainable from this body, via dehydration of clays, etc..).

We will assume an arbitrary 60 day stay time (or mining season) on the body, centred around its aphelion, and near-Hohmann-ellipse transfer orbits out and back..

1. Let us calculate the outbound $\Delta v$ to depart LEO on heliocentric transfer to $1989 \mathrm{ML}$, and return departure $\Delta \mathrm{v}$ required to place the payload in an earth-return trajectory.

1989ML has these orbital parameters:

$\mathrm{q}=1.099 \mathrm{AU} ; \quad \mathrm{Q}=1.445 \mathrm{AU} ; \quad \mathrm{e}=0.1365 ; \mathrm{i}=4.38$ degrees; $\mathrm{a}=1.272 \mathrm{AU}$ 


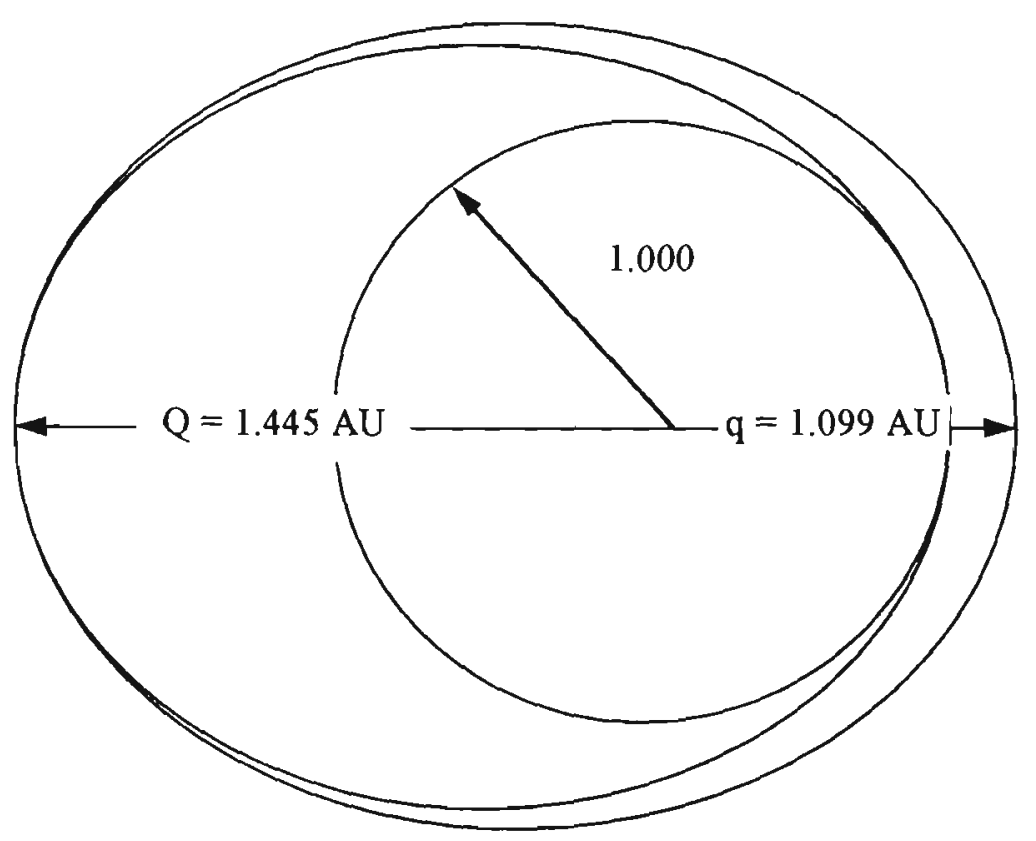

Figure 9.1 "Apollo-type" mission

The outbound velocity requirements $\Delta \mathrm{v}$ outbound, hyperbolic and $\Delta \mathrm{v}$ departure, leo will now be calculated:

The velocity required by a spacecraft at the perihelion of the transfer orbit shown in Fig 9.1 above, is

$\mathrm{v}_{\text {perihelion, transfer }}=\sqrt{\frac{\mu}{1.00}\left[\frac{2(1.445 / 1)}{1+(1.445 / 1)}\right]}$

$\mu_{\operatorname{sun}}=1.33 \times 10^{20} \mathrm{~m}^{3} / \mathrm{s}^{2} ; 1 \mathrm{AU}=1.5 \times 10^{11} \mathrm{~m}$

so $v_{p, t}=\sqrt{\frac{1.33 \times 10^{20}}{1.5 \times 10^{11}}\left[\frac{2.890}{2.445}\right]}$

\section{$=32.37 \mathrm{~km} / \mathrm{sec}$}

Since the circular orbital velocity of the earth is $\mathrm{v}_{\text {earth }}=29.8 \mathrm{~km} / \mathrm{sec}$,

the difference, $\mathrm{v}_{\text {outbound, hyperbolic }}=2.57 \mathrm{~km} / \mathrm{sec}$. 
The question then becomes, "what is the required $\Delta v$ in LEO to give Earth-escape plus sufficient excess energy to provide this hyperbolic velocity?'

$\Delta \mathrm{v}_{\text {departure, leo }}=?$

$\mathrm{v}_{\text {burnout }}{ }^{2}=\mathrm{v}_{\infty}{ }^{2}+\mathrm{v}_{\text {esc }}{ }^{2}$ (from Conservation of Energy, and eqn (11), 4.4.1)

$$
=2.57^{2}+11.2^{2}
$$

$\therefore \mathrm{v}_{\text {bo }}=11.49 \mathrm{~km} / \mathrm{sec}$

Since orbital velocity in LEO is $8.0 \mathrm{~km} / \mathrm{s}, \underline{\Delta v_{\text {departure,leo }}}=11.49-8.0=\underline{3.49 \mathrm{~km} / \mathrm{sec}}$

Note that deep-space rendezvous $\Delta \mathrm{v}$ is small, and is in fact identical to return departure $\Delta v$ calculated in the next section, and is $0.62 \mathrm{~km} / \mathrm{sec}$.

Now we will calculate the return requirement:

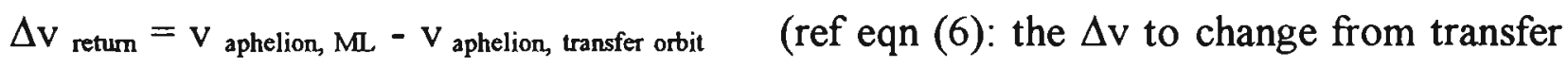
orbit to outer orbit at aphelion is identical (but in opposite direction) to the $\Delta v$ required to change from the outer orbit to the transfer orbit, at aphelion.)

$$
\begin{aligned}
v_{Q, M L} & =\sqrt{\frac{\mu\left(1-e_{M L}\right)}{Q_{M L}}} \quad \text { (from eqn (5), section 4.4.1) } \\
& =\sqrt{\frac{1.33 \times 10^{20}(1-0.136)}{1.445 \times 1.5 \times 10^{11}}} \\
& =23.025 \mathrm{~km} / \mathrm{sec}
\end{aligned}
$$




$$
\begin{aligned}
& \mathrm{v}_{Q, \text { transf }}=\sqrt{\frac{\mu\left(1-e_{t r}\right)}{Q, t r}} \quad \text { (from eqn (5), again) } \\
& {\left[e_{t r}=\frac{Q-q}{Q+q}=\frac{1.445-1}{1.445+1}=0.182\right]} \\
& \mathrm{v}_{Q, t r}=\sqrt{\frac{1.33 \times 10^{20}}{1.445 \times 10^{11}}(0.818)} \\
& \quad=22.404 \mathrm{~km} / \mathrm{sec} .
\end{aligned}
$$

\section{$\therefore \Delta \mathrm{v}_{\text {retum, departure }}=23.025-22.404=0.621 \mathrm{~km} / \mathrm{sec}$ (to decrease heliocentric velocity).}

Note that there may also be an inclination change required, since $\mathrm{i}$ for $1989 \mathrm{ML}$ is at $4.38^{\circ}$ to the ecliptic.

If the line of nodes of the orbits of Earth and 1989ML is coincident with Q, q for the transfer orbit, then no separate deep-space $\Delta \mathrm{v}$ "burn" for inclination change is required. However if the line of nodes is not coincident with the major axis of the transfer orbit, then an inclination change will be required, so as to rotate the plane of the transfer orbit into the ecliptic. At its simplest, when the line of nodes is $90^{\circ}$ away in anomaly from Q, q of transfer orbit, this will require a $\Delta v$ for plane change at the line of nodes, of magnitude $=0.5 \mathrm{~km} / \mathrm{sec}$ per degree of inclination change.

Thus at worst, for 1989ML,

$$
\Delta \mathrm{v}_{\text {inclin change }}=2.19 \mathrm{~km} / \mathrm{sec} \text {, for } 4.38^{\circ}
$$

Note that the plane-change $\Delta v$ dominates!

(This also applied on the outbound journey, although it was not mentioned above.)

Note that in general, this $\Delta \mathrm{v}_{\text {inclin }}$ will not take place at the same time as asteroid departure, i.e., $\Delta \mathrm{v}_{\text {retum }}$, but at some later time, and will therefore be additive, i.e., 


$$
\Delta \mathrm{v}_{\text {retum, total }}=2.19+0.62=2.81 \mathrm{~km} / \mathrm{sec}
$$

If and only if the departure from $1989 \mathrm{ML}$ can be delayed after aphelion long enough to coincide with its passage through the line of nodes, can the inclination change and the transfer orbit "burn" occur together. If that is possible, then

$$
\Delta \mathrm{v}_{\text {return,total }}=\sqrt{ }\left(0.62^{2}+2.19^{2}\right)=2.28 \mathrm{~km} / \mathrm{sec} \text {. (from Pythagoras). }
$$

Thus for $1989 \mathrm{ML}$, taking plane change into account, $\Delta \mathrm{v}_{\text {retum }}=2.8 \mathrm{~km} / \mathrm{sec}$. at worst.

2. Let us assume use of a solar thermal rocket, with exhaust temp. of $2400 \mathrm{~K}$. (This operating temperature requires a Rhenium nozzle).

$\mathrm{v}_{\mathrm{e}}=270 \sqrt{\frac{\bar{T}}{M W}} \cong 3100 \mathrm{~m} / \mathrm{s} ;$ since $\mathrm{v}_{\mathrm{e}}=10 \times \mathrm{I}_{\mathrm{sp}}, \mathrm{I}_{\mathrm{sp}}=310$ seconds

From the Rocket Equation, $\Delta \mathrm{v}=\mathrm{v}_{\mathrm{e}} \times \ln \left[\frac{M_{\text {start }}}{M_{\text {finish }}}\right]$, we get alternatively:

$\mathrm{M}_{\text {start }}=\mathrm{M}_{\text {finish }} \times e^{\Delta v / v_{\mathrm{e}}} ;$ for $\mathrm{v}_{\mathrm{e}}=3.1$ and $\Delta \mathrm{v}=2.8 \mathrm{~km} / \mathrm{sec}$, respectively, $e^{\Delta v / v_{c}}=2.47$ for the above $\Delta v_{\text {return }}$.

So $\mathrm{M}_{\text {start }}=1000 \times 2.47=2470$ tonnes at departure from asteroid.

Thus the volatile mass used as propellant is 1470 tonnes.

3. What energy is required to produce 2500 tonnes of water in (say) 60 days, assuming $1989 \mathrm{ML}$ is a carbonaceous chondrite - type asteroid, and that volatiles recovery is $10 \%$ of treated mass?

The implied regolith processing rate is 18 tonnes per hour. 
The heating power needed to raise the temperature of this mass from $0^{\circ} \mathrm{C}$ to $600^{\circ} \mathrm{C}$, given specific heat of regolith of $\cong 1 \mathrm{~J} / \mathrm{gm} . \mathrm{K}$, is 3 Megawatts (without use of heat exchanger).

With heat recovery from tailings prior to discarding them, we might reduce heat input requirement to (say) $1 \mathrm{MW}$. This should be preferably via a heat engine rather than a heat exchanger, because of the massive nature of heat exchangers, and the necessity in any case for a supply of mechanical energy.

4. Rocket propulsion power and propellant usage:

$\mathrm{dm} / \mathrm{dt}=\frac{\text { power in }}{\text { spec enthalpy for ice } \rightarrow \text { steam at } 2400 \mathrm{~K}}$

$=\frac{1 \times 10^{6} \mathrm{~W}}{\approx 8000 \times 10^{3} \mathrm{~J} / \mathrm{kg}}$

$\cong 0.125 \mathrm{~kg} / \mathrm{sec}$

Therefore Thrusting time $=\left(\frac{1500 \times 10^{3}}{0.125}\right)$ seconds

$$
=130 \text { days }
$$

And the thrust is $\mathrm{dm} / \mathrm{dt} \times \mathrm{v}_{\mathrm{e}}$

$$
\begin{aligned}
& =0.125 \times 3100 \\
& =387.5 \text { Newtons }
\end{aligned}
$$

5 Now we can construct a burn time / fuel used / $\Delta \mathrm{v}$ plot for $1989 \mathrm{ML}$, using the Rocket Equation: 


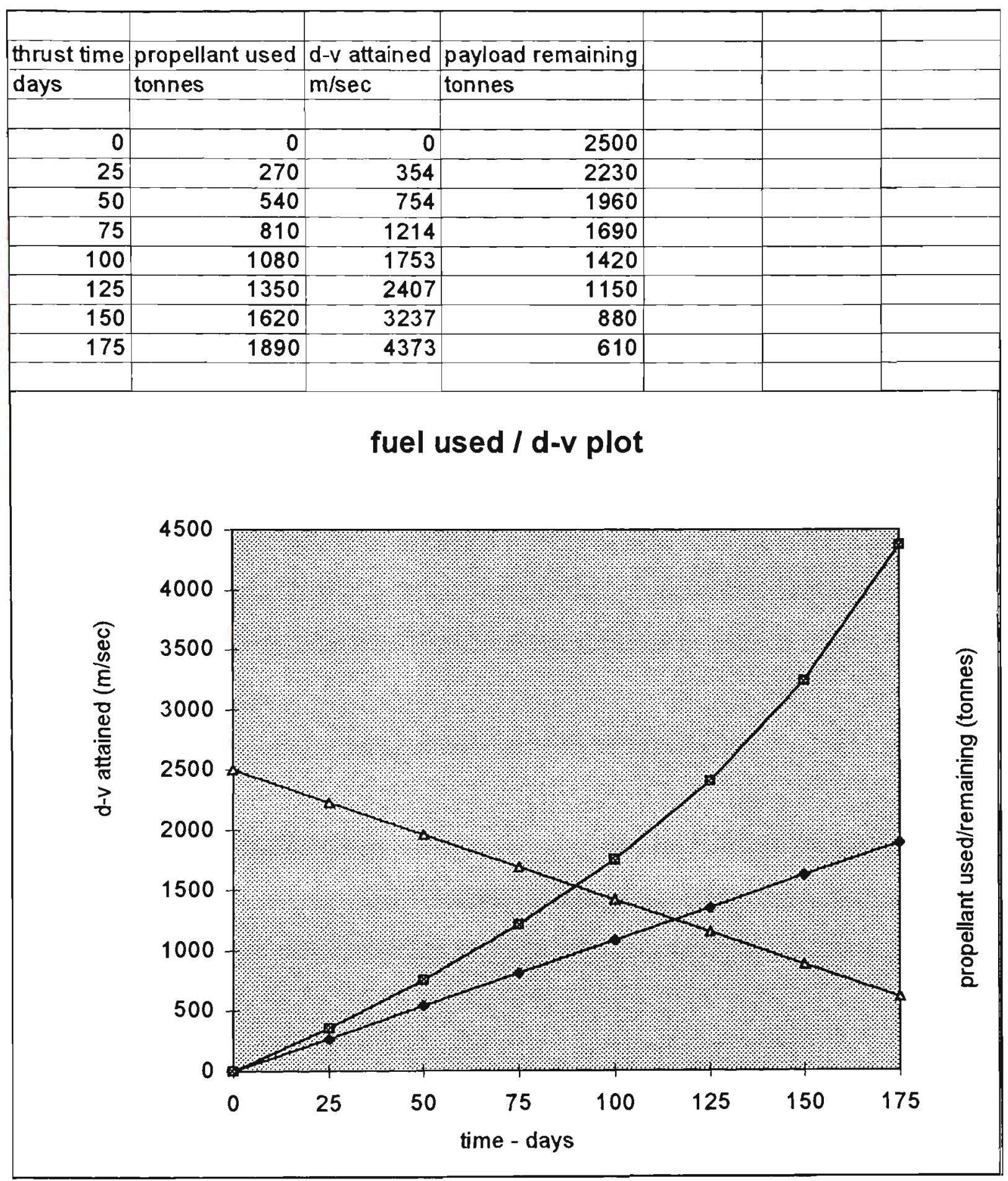

Figure 9.2 Fuel-use vs delta-v graph

NB: Period T of transfer orbit is $=(a) 3 / 2$

$$
\begin{aligned}
& =1.352 \text { years } \\
& =493 \text { days }
\end{aligned}
$$

From this we see that $2.8 \mathrm{~km} / \mathrm{s}$ is attained in about 135 days of thrusting, with almost exactly 1000 tonnes remaining. 
This is assumed to bring the payload into an orbit which is tangent to the earth's, at its perihelion. Capture is assumed to be assisted by lunar gravity flyby, and will be into a highly-elliptical earth orbit (HEEO).

Hyperbolic velocity $v_{\infty}$ at time of capture will be

$$
\begin{array}{ll}
\mathrm{v}_{\mathrm{p}}, \mathrm{t} & =32.37 \mathrm{~km} / \mathrm{s} \\
\mathrm{v}_{\text {earth }} & =30 \mathrm{~km} / \mathrm{s} \\
\therefore \mathrm{v}_{\infty} & =2.37 \mathrm{~km} / \mathrm{s}
\end{array}
$$

Of this, $\sim 1.5 \mathrm{~km} / \mathrm{s}$ can be subtracted in a single lunar flyby (ref O'Leary), leaving a further $0.9 \mathrm{~km} / \mathrm{s}$ to be removed by propulsive braking, in order to achieve Earth-capture. From the Rocket Equation, this will reduce remaining propellant mass from 1000 to $\mathbf{7 4 8}$ tonnes. Retro-thrusting would start $\sim 25$ days pre-arrival.

The final transfer from HEEO to LEO will cost a further $\Delta \mathrm{v}$ of $\cong 2.5 \mathrm{~km} / \mathrm{s}$.

This could be accomplished using aerobraking - (but this would imply robotic fabrication of same on the asteroid - another level of complexity) - or could again be achieved propulsively, unfortunately using still more of the diminishing payload:

$$
\begin{aligned}
\text { M finish } & =\quad \text { start } \times \mathrm{e}^{-\Delta v / v e} \\
& =750 \times 0.446 \\
& =335 \text { tonnes !! }
\end{aligned}
$$

If we assume sale in HEEO, then 750 tonnes, at (say) $\$ 4000 / \mathrm{kg}=\$ 3 \times 10^{9}$

Time since drawdown of funds $=\mathrm{T}_{\text {transfer }}$ plus pre-launch construction and preparation time is taken to be approx 2.5 years; then

$$
\mathrm{NPV}=\frac{R}{(l+i)^{n}}-\text { Capital invested }
$$




$$
\begin{gathered}
=\quad \frac{3 \times 10^{9}}{(1+0.3)^{2.5}}-\text { Capital } \quad \text { (assume } 30 \% \text { cost of loan money) } \\
=\quad \$ 1500 \times 10^{6} \text { - initial Capital. }
\end{gathered}
$$

Hence initial capital must be less than $\$ 1500$ million for NPV to be positive, and the project to be feasible.

However for delivery into LEO, where a lower value (of $\$ 1000 / \mathrm{kg}$ ) is assumed to apply, and only 335 tonnes is left over for sale, the sale income is $\$ 335$ million or an NPV of approx $\$ 170$ million. This sets the limit on maximum allowable initial capital for the project giving delivery into LEO.

The finding that delivery into high Earth orbit would be about ten times more lucrative is interesting, but in the absence of any likely large-scale market, is quite hypothetical. 


\subsection{Example \#2 : 1982DB Nereus ("Apollo-Type") mission}

Return of approx 1000 tonnes of volatiles from 1982DB Nereus: assume that approx $10 \%$ of the mass of the regolith is readily extracted volatile $\left(\mathrm{H}_{2} \mathrm{O}, \mathrm{CO}_{2}\right.$, etc. $)$. Assume an aphelion mining season of 60 days.

1982DB Nereus has the following orbital parameters:

Table 9.1 Nereus orbital parameters

\begin{tabular}{|c|c|c|c|c|c|}
\hline $\begin{array}{c}\text { Perihelion } \\
\mathrm{q}(\mathrm{AU})\end{array}$ & $\begin{array}{c}\text { Aphelion } \\
\mathrm{Q}(\mathrm{AU})\end{array}$ & $\begin{array}{c}\text { eccent } \\
\mathrm{e}\end{array}$ & $\begin{array}{c}\text { inclin } \\
\mathrm{i}(\mathrm{deg})\end{array}$ & $\begin{array}{c}\text { s.m.a } \\
\mathrm{a}(\mathrm{AU})\end{array}$ & $\begin{array}{c}\text { period } \\
\underline{\mathrm{T}(\mathrm{yrs})}\end{array}$ \\
\hline 0.953 & 2.025 & 0.36 & 1.4 & 1.489 & 1.82 \\
\hline
\end{tabular}

We now wish to calculate the $\Delta v$ required to depart from with sufficient energy to give the required $v$ hyperbolic to enter the transfer orbit to Nereus.

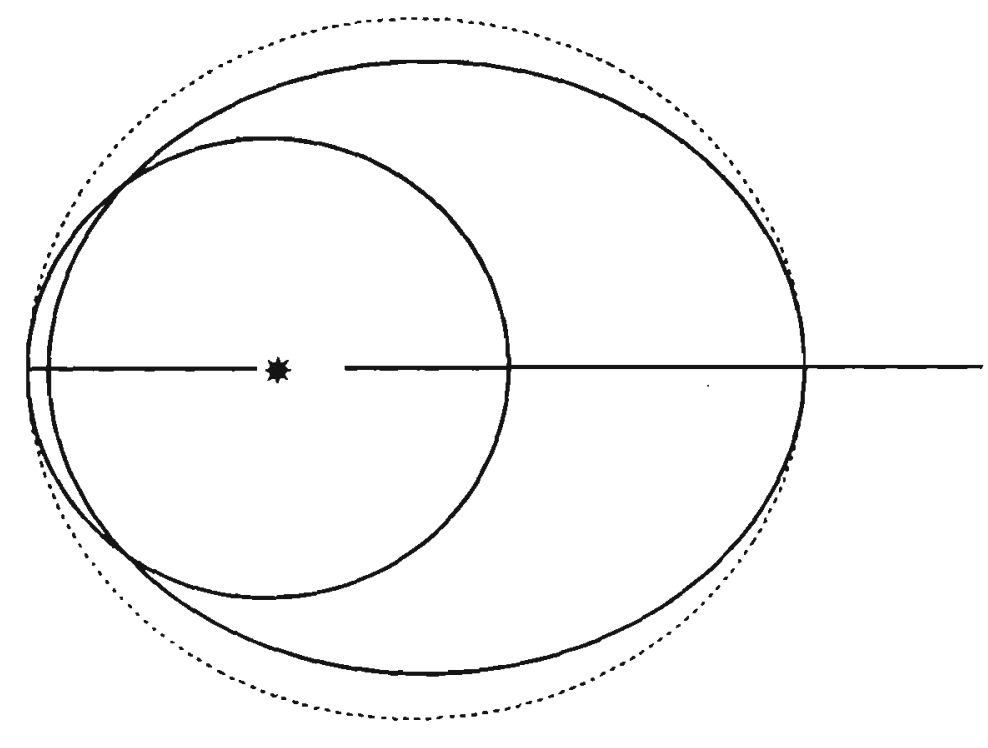

Figure 9.3 "Apollo-type" mission, example \#2 


$$
\begin{gathered}
\mathbf{v}_{\mathrm{p}, \mathrm{t}}=\sqrt{\frac{\mu\left(2\left(r_{a 2} / r_{p 1}\right)\right)}{r_{p}\left(1+\left(r_{a 2} / r_{p 1}\right)\right)}} \\
=\sqrt{\frac{1.33 \times 10^{20}}{1.5 \times 10^{11}} \frac{(2 \times 2.025 / 1)}{(3.025)}} \\
=34.45 \mathrm{~km} / \mathrm{sec}
\end{gathered}
$$

Therefore $\mathrm{v}_{\text {hyper }}=34.45-29.80=4.65 \mathrm{~km} / \mathrm{sec}$

$$
\begin{aligned}
\Delta \mathrm{v}_{\text {depart leo }}=\sqrt{(11.2)^{2}+(4.65)^{2}}-8.0 & \\
& =12.13-8.0=4.13 \mathrm{~km} / \mathrm{sec}
\end{aligned}
$$

Deep - Space rendezvous velocity $\Delta v$ Ds must now be calculated:

$$
\begin{aligned}
\Delta \mathrm{v}_{\mathrm{DS}} & =\Delta \mathrm{v}_{\mathrm{a}, \text { trans }}=\mathrm{v}_{\mathrm{a}, \text { trans }}-\mathrm{v}_{\mathrm{a}, \mathrm{DB}} \\
\mathrm{v}_{\mathrm{a}, \text { trans }} & =34.45 \times \frac{1}{2.025} \text { from Conservation of Angular Momentum } \\
& =17.01 \mathrm{~km} / \mathrm{sec} \\
& =\sqrt{\frac{\mu}{Q_{D B}}\left(1-e_{D B}\right)} \\
\mathrm{v}_{\mathrm{a}, \mathrm{DB}} & \text { (from eqn (5)) } \\
& =\sqrt{\frac{1.33 \times 10^{20}}{2.025 \times 1.5 \times 10^{11}}(1-0.36)} \\
& =16.74 \mathrm{~km} / \mathrm{sec} \\
\text { So } \underline{\Delta \mathrm{v}_{\mathrm{DS}}} & =17.01-16.74
\end{aligned}
$$

This is also the $\Delta v$ required, at aphelion, to inject into an Earth-return transfer orbit. 
Return trajectory: Note that Nereus' orbit is inclined $1.43^{\circ}$ to Earth's, so there will be a small plane change needed, (unless departure is at the line of nodes!)

So $\Delta \mathrm{v}_{\text {inc }}=1.43 \times 0.5=0.71 \mathrm{~km} / \mathrm{s}$

The total return $\Delta v$ is $5.63 \mathrm{~km} / \mathrm{s} \quad(=0.27+4.65+0.71)$. This is because $\Delta v$ for departure from the asteroid is equal to $\Delta v$ for rendezvous at arrival; and $v_{\infty}$ hyperbolic velocity upon arrival will be equal to the departure $\Delta v_{\text {hyper }}$ calculated earlier; $0.71 \mathrm{~km} / \mathrm{s}$ is the plane-change requirement.

- the rocket equation implies a starting volatiles (propellant) mass of

$$
\begin{array}{rlrl}
\mathbf{M}_{\text {st }} & = & \mathbf{M}_{\mathbf{f}} \times \mathrm{e}^{\Delta \mathbf{v} / v e} \\
& = & 1000 \times 6.15 \\
& =\quad 6150 \text { tonnes }
\end{array}
$$

To collect 6000 tonnes of volatiles, it is assumed that 60,000 tonnes of regolith must be processed. To do this in 60 days implies $1000 \mathrm{tpd}$

$$
\begin{aligned}
& \cong \quad 40 \text { tonnes per } \mathrm{hr} \\
& =\quad 11 \mathrm{~kg} / \mathrm{sec}
\end{aligned}
$$

To heat 40 tonnes per hour to (say) $600^{\circ} \mathrm{C}$ from $0^{\circ} \mathrm{C}$ will require approx $6 \mathrm{MW}$.

(Less if Heat Exchanger / Recuperator is used)

If this material requires to be crushed and milled to $500 \mu \mathrm{m}$, then

Grinding Power requirement $=$ Bond Work Index $\times$ Mass flow rate

Assume Wi = $5 \mathrm{kWhr} /$ tonne;

at 40 tonnes $/ \mathrm{hr}$, Grinding Power $=40 \frac{\text { tonnes }}{\text { hour }} \times 5 \frac{\mathrm{kWhr}}{\text { tonne }}$

$$
=200 \mathrm{~kW}
$$


Note that this mechanical power requirement is small compared with the thermal power requirement of $6 \mathrm{MW}$.

The thrusting time required to achieve any given $\Delta \mathrm{v}$ on the return depends on Heat Power and thus on $\mathrm{dm} / \mathrm{dt}$.

For Heat Power to the solar thermal rocket of (say) 3MW, and specific enthalpy of approx $8000 \times 10^{3} \mathrm{~J} / \mathrm{kg}$ to heat water to $2400 \mathrm{~K}$,

$$
\mathrm{dm} / \mathrm{dt}=0.375 \mathrm{~kg} / \mathrm{sec}
$$

At $0.375 \mathrm{~kg} / \mathrm{sec}$, how long will it take to consume propellant mass of 5000 tonnes (leaving 1000 tonnes of payload)?

$$
\begin{aligned}
\text { thrusting time } & =\left(\frac{4 \times 10^{6}}{0.375}\right) \text { seconds } \\
& =123 \text { days }
\end{aligned}
$$

Approximately half of the remaining volatiles payload must then be used to drop into low earth orbit, as calculated in the previous example, unless aerobraking or another method of killing excess velocity is used.

The total time from launch for the resource return project is approximately 2.5 years; therefore, as was the case for the previous example, NPV for material delivery into LEO is $(\$ 1000 / \mathrm{kg} \times 500,000 \mathrm{~kg} \times 0.5)=\$ 250$ million, assuming $30 \%$ interest rate.

Obviously, anything which reduces the requirement for propulsive $\Delta v$ for earth return and injection into LEO; and any possibility of a less onerous cost of money, would assist the economics of these missions greatly. 


\subsection{Example Project \# 3 : 1989UQ (“Aten-Type”) mission}

let us consider a mission to an Aten, 1989UQ, again assuming that volatiles can be recovered by thermal processing of regolith material to extract $\mathrm{H}_{2} \mathrm{O}$ from clays etc.

1989UQ has the orbital parameters:

Table 9.2 Orbital parameters, $1989 U Q$

\begin{tabular}{|c|c|c|c|c|}
\hline $\begin{array}{c}\text { Perihelion } \\
\mathrm{q}(\mathrm{AU})\end{array}$ & $\begin{array}{c}\text { Aphelion } \\
\mathrm{Q}(\mathrm{AU})\end{array}$ & $\begin{array}{c}\text { eccentricity } \\
\mathrm{e}\end{array}$ & $\begin{array}{c}\text { inclination } \\
\mathrm{i}(\mathrm{deg})\end{array}$ & $\begin{array}{c}\text { semi-major axis } \\
\mathrm{a}(\mathrm{AU})\end{array}$ \\
\hline 0.67 & 1.16 & 0.26 & 1.28 & 0.915 \\
\hline
\end{tabular}

Rendezvous can be made either at perihelion or at aphelion.

We will calculate $\Delta v$ out and $\Delta v$ return for both cases.

\section{Aphelion rendezvous}

$$
\begin{aligned}
& \Delta \mathrm{v} \text { outbound, } \mathrm{v}_{\text {hyperbolic }}=\mathrm{v}_{\text {peri, transfer }}-\mathrm{v}_{\text {earth }} \\
& {\left[\mathrm{v}_{\text {earth }}=30 \mathrm{~km} / \mathrm{s}\right]} \\
& \mathrm{v}_{\text {perihelion, transfer }}=\sqrt{\frac{\mu}{r_{\text {peri }}\left[\frac{2(Q / 1)}{1+(Q / 1)}\right]}} \\
& =\quad 30.86 \mathrm{~km}
\end{aligned}
$$


So $\mathrm{v}_{\text {hyperbolic }}=0.86 \mathrm{~km} / \mathrm{s}$, for aphelion rendezvous

$\Delta v$ departure, LEO (i.e., the velocity increment required in LEO to give the necessary hyperbolic velocity to enter the transfer orbit desired) is LEO departure velocity - LEO orbital velocity; LEO departure velocity is that required by Conservation of Energy to give the hyperbolic excess velocity needed for the transfer orbit.

$$
\begin{aligned}
& \text { LEO departure velocity, } \mathrm{v} \text { burn out }=\sqrt{11.2^{2}+0.86^{2}} \\
& =\sqrt{125.44+0.74}=11.23 \mathrm{~km} / \mathrm{s} \\
& \text { So } \Delta \mathrm{v}_{\text {dep, leo }}=11.23-8.0=3.23 \mathrm{~km} / \mathrm{s} \text { (for aphelion rendezvous) } \\
& \text { Return: } \Delta \mathrm{v}_{\text {retum, aphelion }}=\left[v_{\text {aph, } U Q}-v_{\text {aph,transferorbit }}\right.
\end{aligned}
$$

Note that transfer orbit has higher perihelion than 1989UQ, $\therefore \mathrm{v}_{\text {aphel,transfer }}>\mathrm{v}_{\text {aphel,UQ. }}$

$$
\begin{aligned}
\mathrm{V}_{\text {aphel, UQ }} & =\sqrt{\frac{\mu}{Q_{U Q}}\left(1-e_{U Q}\right)} \quad \text { from eqn (5) 4.4.1 } \\
& =\sqrt{\frac{1.83 \times 10^{20}}{1.5 \times 10^{11} \times 1.16}(1-0.26)} \\
& =\sqrt{\frac{1.33 \times 10^{20} \times 0.74}{1.5 \times 10^{11} \times 1.16}} \\
& =23.78 \mathrm{~km} / \mathrm{s} \\
\mathrm{V}_{\text {aphel, transfer }} & =\sqrt{\frac{1.33 \times 10^{20}}{1.5 \times 10^{11} \times 1.16}\left(1-e_{\text {transfer }}\right)} \\
\mathrm{e}_{\text {transfer orbit }} & =\frac{Q-q}{Q+q}=\frac{0.16}{2.16}= \\
& =\frac{1.16-1}{1.16+1}=0.074
\end{aligned}
$$


So $\mathrm{v}_{\text {aphel, transfer }}=26.60 \mathrm{~km} / \mathrm{s}$

So $\Delta \mathrm{v}_{\text {return }}=26.60-23.78=\underline{2.82 \mathrm{~km} / \mathrm{s}}$

That is, for return from aphelion of $1989 \mathrm{UQ}$, a departure $\Delta v$ of $2.82 \mathrm{~km} / \mathrm{s}$ is needed, in order to increase heliocentric velocity in orbit, and intersect Earth orbit.

Perihelion rendezvous: Outbound hyperbolic velocity, $\mathrm{v}_{\infty}=\mathrm{v}_{\mathrm{e}}-\mathrm{v}_{\text {aphel, transfer orbit }}$

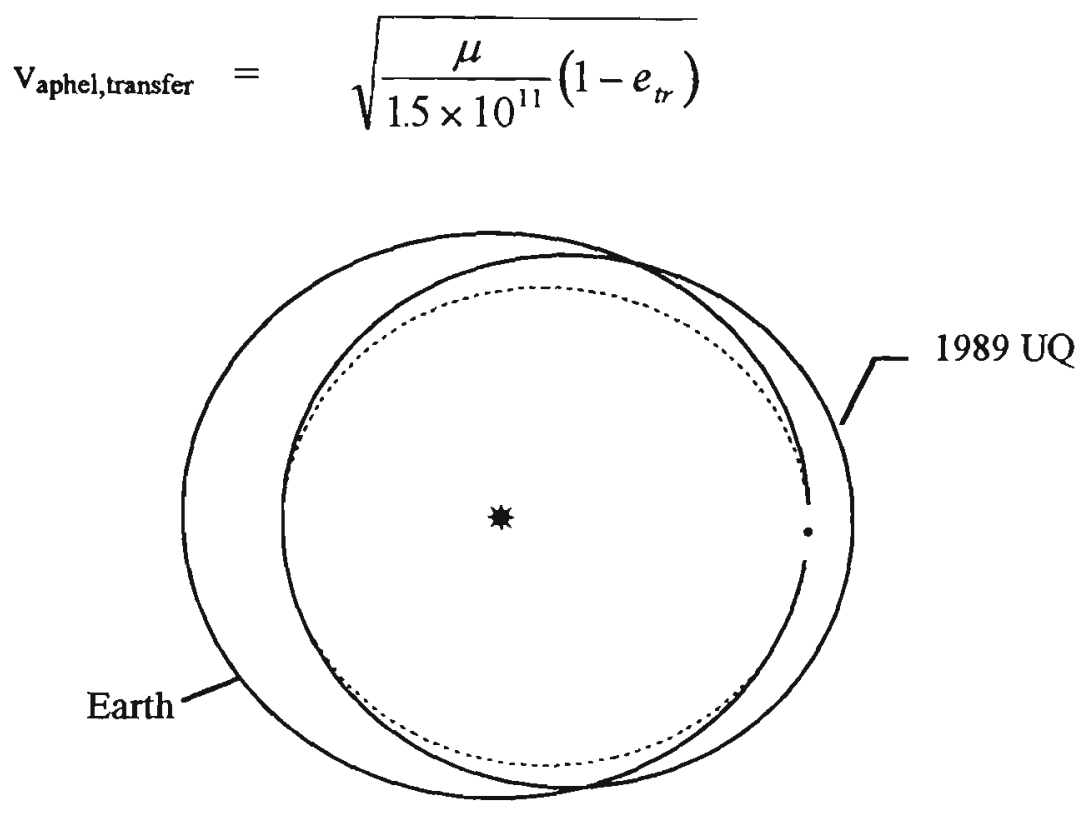

Figure 9.4 "Aten-type" mission

$$
\begin{aligned}
\mathrm{e}_{\text {transfer }} & =\frac{Q t-q t}{Q t+q t}=\frac{1.0-0.67}{1.0+0.67} \\
& =\frac{0.33}{1.67}=0.198 \\
\mathrm{~V}_{\text {aphel, transfer }} & =26.67 \mathrm{~km} / \mathrm{s}
\end{aligned}
$$

Since Earth's circular velocity is $30 \mathrm{~km} / \mathrm{s}$, then $\mathrm{v}_{\infty}=3.33 \mathrm{~km} / \mathrm{s}$ for perihelion rendezvous.

$\Delta \mathrm{v}_{\text {departure, leo }}=\sqrt{11.2^{2}+3.33^{2}}-8.0$ 


$$
\begin{aligned}
& =\sqrt{125.44+11.09}-8.0 \\
& =11.68 \mathrm{~km} / \mathrm{s}-8.0 \mathrm{~km} / \mathrm{sec} \\
& =\quad 3.68 \mathrm{~km} / \mathrm{sec}
\end{aligned}
$$

Return:

$$
\begin{aligned}
\mathrm{v}_{\text {peri, UQ }} & =\sqrt{\frac{\mu}{Q_{U Q}}\left(1-e_{U Q}\right)} \\
& =\sqrt{\frac{1.33 \times 10^{20}}{0.67 \times 1.5 \times 10^{11}}(1+0.26)} \\
& =40.83 \mathrm{~km} / \mathrm{s} \\
\mathrm{v}_{\text {peri, trans }} & =\sqrt{\frac{\mu}{q_{t r}}\left(1+e_{t r}\right)} \\
& {\left[e_{t r}=\frac{Q_{t r}-q_{t r}}{Q_{t r}+q_{t r}}=\frac{1-0.67}{1.67}=0.197\right] } \\
\mathrm{v}_{\text {peri, trans }} & =39.80 \mathrm{~km} / \mathrm{s}
\end{aligned}
$$

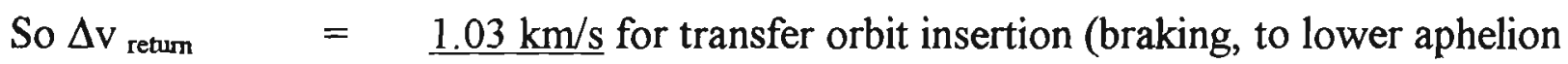
to earth orbit tangent). This is a surprisingly low figure!

To calculate hyperbolic velocity on arrival at earth orbit, use Conservation of Angular Momentum: $\quad \mathrm{v}_{\text {peri, trans }} \times \mathrm{q}_{\mathrm{tr}}=\quad \mathrm{V}_{\mathrm{ap}}$, transfer $\times \mathrm{Q}_{\mathrm{tr}}$

$$
\mathrm{V}_{\mathrm{aph}, \text { trans }}=\frac{39.80 \times 0.67}{1.00}=26.67 \mathrm{~km} / \mathrm{sec}
$$
So $\quad v_{\text {hyp }}$
$=30-26.67$
$=$
$3.33 \mathrm{~km} / \mathrm{sec}$

(in agreement with calculation for $\mathrm{v}$ outbound) 
$\Delta v$ for inclination change: This depends on where i change occurs, and heliocentric velocity at that point.

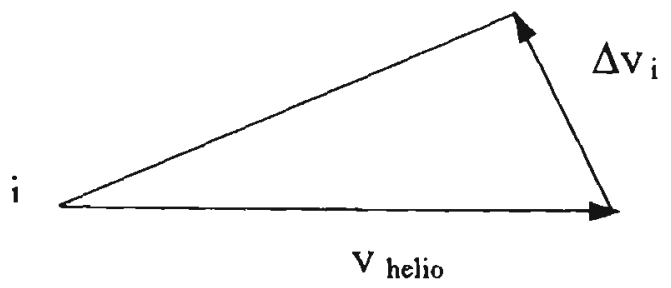

$$
\begin{aligned}
& \tan \mathrm{i}=\quad \sin \mathrm{i}=\frac{\Delta v_{i}}{v_{\text {heliocentic }}} \quad \therefore \Delta \mathrm{v}_{\mathrm{i}}=\mathrm{v}_{\text {helio }} \times \sin \mathrm{i} \\
& \text { for } \mathrm{v}_{\text {helio }}=30 \mathrm{~km} / \mathrm{sec} \\
& \Delta \mathrm{v}_{\text {incl }}=(0.52 \times \mathrm{i}) \mathrm{km} / \mathrm{s}
\end{aligned}
$$

So a $1.28^{\circ}$ plane change implies $\Delta \mathrm{V}_{\text {incl }}=\underline{0.665 \mathrm{~km} / \mathrm{s}}-$ if accomplished at $30 \mathrm{~km} / \mathrm{sec}$ heliocentric velocity.

So $\Delta \mathrm{v}$ for return to earth transfer is $1.03+0.67=1.7 \mathrm{~km} / \mathrm{s}$. Using the $1989 \mathrm{ML}$ calculations, we see that this can be achieved in 100 days, with a payload injected into transfer orbit of a little over half of the start mass (i.e., for the 5000 tonne case, 2500 tonnes onto Earth-return trajectory).

Some of this mass will necessarily be used in propulsive braking to attain Earth-capture.

So, for this and other Atens (as noted in Shoemaker and Helin, 1978), there is a choice between perihelion rendezvous and aphelion rendezvous, with a half-period mining stay, then a return trajectory departing at aphelion or perihelion, repectively.

Calling the "Aphelion rendezvous / perihelion-departure return" case Mission 1, and the "Perihelion rendezvous / aphelion-departure return" case Mission 2, the following comparison is found: 
Table 9.3 Aten Missions comparison: 1989UQ

\begin{tabular}{|c|c|c|c|c|c|}
\hline & $\begin{array}{l}\text { Earth } \\
\text { departure } \\
\Delta \mathrm{v}_{\text {leo, depart }}\end{array}$ & $\begin{array}{l}\text { asteroid arrival } \\
\text { (aphelion) } \\
\Delta v_{\text {Deep Space }}\end{array}$ & $\begin{array}{l}\text { asteroid } \\
\text { departure } \\
\text { (perihelion) }\end{array}$ & $\begin{array}{l}\text { Plane } \\
\text { change }\end{array}$ & $\begin{array}{l}\text { Earth arrival } \\
\text { (capture only) } \\
v_{\infty}\end{array}$ \\
\hline Mission 1 & 3.23 & 2.82 & 1.03 & 0.67 & 3.33 \\
\hline & $\begin{array}{l}\text { Earth } \\
\text { departure }\end{array}$ & $\begin{array}{l}\text { asteroid arrival } \\
\text { (perihelion) }\end{array}$ & $\begin{array}{l}\text { asteroid } \\
\text { departure } \\
\text { (aphelion) }\end{array}$ & $\begin{array}{l}\text { Plane } \\
\text { change }\end{array}$ & $\begin{array}{l}\text { Earth arrival } \\
\text { (capture only) }\end{array}$ \\
\hline Mission 2 & 3.68 & 1.03 & 2.82 & 0.67 & 0.86 \\
\hline
\end{tabular}

Note the outbound propulsive requirements are easier for Mission 2 (total outbound $\Delta \mathrm{v}$ of $4.71 \mathrm{~km} / \mathrm{s}$ versus $6.05 \mathrm{~km} / \mathrm{s}$ for Mission 1). The return trajectory comparison is interesting: Mission 1 return departure $\Delta v$ is approx $1 \mathrm{~km} / \mathrm{s}$ versus approx $2.8 \mathrm{~km} / \mathrm{s}$ for Mission 2, but the hyperbolic return velocity for Mission 1 is $3.3 \mathrm{~km} / \mathrm{s}$ versus $0.86 \mathrm{~km} / \mathrm{s}$ for Mission 2, which is much less demanding in propellant useage, and may well be amenable to lunar flyby capture.

Mission 2 is clearly the less demanding case, having a total return $\Delta \mathrm{v}$ requirement to Earth-capture of $4.35 \mathrm{~km} / \mathrm{s}$.

NPV for this case will assisted by the extra time available for mining stay $(T / 2=160$ days) which thus reduces the mass required for mining equipment and also reduces the power requirement for its operation.

Additionally of advantage for NPV is the relatively short mission time, which is approximately 17 months from launch. 


\subsection{Example \# 4: Mission to an 'Arjuna', 1991VG.}

'Arjunas' are very small (eg diameter $=20 \mathrm{~m}$ ), have very "Earth-like" orbits, and have very long synodic periods, so either outbound or return trajectory will either be non Hohmann or will require phasing orbit; $\Delta v^{\prime}$ s outbound and return are under $1 \mathrm{~km} / \mathrm{s}$. 1991VG has the orbital parameters:

Table 9.4 Orbital parameters, $1991 \mathrm{VG}$

\begin{tabular}{|c|c|c|c|c|}
\hline $\begin{array}{c}\text { Perihelion } \\
\mathrm{q}(\mathrm{AU})\end{array}$ & $\begin{array}{c}\text { Aphelion } \\
\mathrm{Q}(\mathrm{AU})\end{array}$ & $\begin{array}{c}\text { eccentricity } \\
\mathrm{e}\end{array}$ & $\begin{array}{c}\text { inclination } \\
\mathrm{i}(\mathrm{deg})\end{array}$ & $\begin{array}{c}\text { semi major axis } \\
\mathrm{a}(\mathrm{QU})\end{array}$ \\
\hline 0.975 & 1.077 & 0.049 & 1.45 & 1.026 \\
\hline
\end{tabular}

$\Delta v$ to depart Earth to rendezous with $1991 \mathrm{VG}$ at its aphelion is:

$$
\begin{aligned}
v_{p_{t}} & =\sqrt{\frac{\mu}{q_{t}}\left(1+e_{t}\right)} \\
& =\frac{1.077-1}{1.077+1}=\frac{0.077}{2.077}=0.037 \\
& =\sqrt{\frac{1.33 \times 10^{20}}{1.5 \times 10^{11} \times 1}(1+0.037)} \\
& =30.32 \mathrm{~km} / \mathrm{sec} \\
\therefore \Delta \mathrm{v}_{\text {outbound }} & =30.32-29.80
\end{aligned}
$$




$$
=\quad 11.21-8.0=3.21 \mathrm{~km} / \mathrm{sec}
$$

$\Delta v$ to depart 1991VG on return, for Earth-transfer-injection -

$$
\begin{aligned}
& \Delta \mathrm{V}=\mathrm{v}_{\mathrm{at}} \quad-\quad \mathrm{v}_{\mathrm{aVG}} \\
& \mathrm{v}_{\mathrm{at}}=\sqrt{\frac{\mu}{Q_{V G}}\left[\frac{2}{1+\left(Q_{V G} / 1\right)}\right]} \\
& =\sqrt{\frac{1.33 \times 10^{20}}{1.077 \times 1.5 \times 10^{11}}\left[\frac{2}{1+1.077}\right]} \\
& =28.16 \mathrm{~km} / \mathrm{sec} \\
& v_{\mathrm{aVG}}=\sqrt{\frac{\mu}{1.077 \times 1.5 \times 10^{11}}(1-0.049)} \\
& =27.98 \mathrm{~km} / \mathrm{sec}
\end{aligned}
$$

So $\Delta \mathrm{v}_{\text {return }}=0.180 \mathrm{~km} / \mathrm{sec}$

Obviously, this is a very small velocity change. In addition, we have already calculated that the $v$ hyp on Earth-arrival is $\approx 0.5 \mathrm{~km} / \mathrm{sec}$., easily low enough for capture and insertion into HEEO by a lunar flyby manoeuvre.

With these objects, mining season is not constrained by the need to depart near aphelion or perihelion, so mining rate can be quite slow; also total propellant requirement is small. This implies a much smaller equipment mass budget than calculated for the "Apollotype" cases discussed earlier.

At $0.1 \mathrm{~kg} / \mathrm{sec}$, Mining Rate $=8.6$ tonnes $/$ day; the formula,

Throughput $=200 \times$ Equipment Mass, gives equipt mass $=50 \mathrm{~kg}$ !

The equipment is dwarfed in the Mass Budget by collector bag (still approx 1.0 tonnes). 
Total system mass could therefore easily be less than 2.0 tonnes.

Thus, in an engineering sense, the Arjunas will be the most easily accessible bodies; the question which is unanswered is whether there is any loose regolith on their surfaces, amenable to mechanical collection (or indeed whether any of them are "ice bodies"). 


\subsection{Example \#5: Wilson-Harrington Volatiles Return}

Asteroid 1979VA Wilson-Harrington is now believed to be a dormant comet, because the discovery plate taken in 1949 showed a tail.

Its orbital parameters are:

Table 9.5 Orbital parameters, Wilson-Harrington

\begin{tabular}{|c|l|l|l|l|l|}
\hline $\begin{array}{c}\text { Perihelion } \\
\mathrm{q}(\mathrm{AU})\end{array}$ & $\begin{array}{c}\text { Aphelion } \\
\mathrm{Q}(\mathrm{AU})\end{array}$ & $\begin{array}{c}\text { eccentricity } \\
\underline{\mathrm{e}}\end{array}$ & $\begin{array}{c}\text { inclination } \\
\mathrm{i}(\mathrm{deg})\end{array}$ & $\begin{array}{c}\text { semi major axis } \\
\underline{\mathrm{a}}(\mathrm{QU})\end{array}$ & $\begin{array}{c}\text { Period } \\
\text { years }\end{array}$ \\
\hline 0.996 & 4.286 & 0.622 & 2.78 & 2.641 & 4.29 \\
\hline
\end{tabular}

What departure $\Delta \mathrm{v}$ is required, in the optimum circumstance, for aphelion rendezvous?

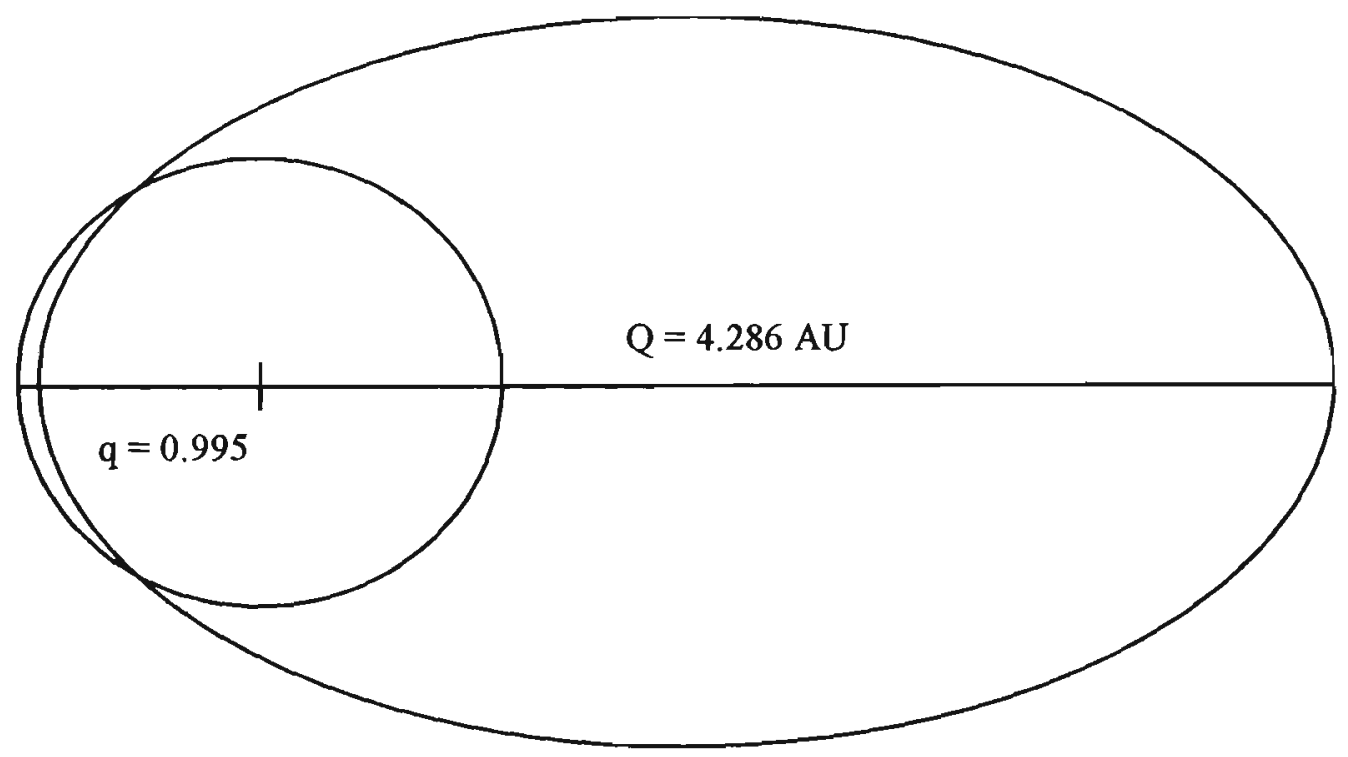

Figure 9.5 Wilson-Harrington orbit

We require to calculate $\mathrm{v}_{\text {outbound, hyperbolic }}$ and $\Delta \mathrm{v}$ departure, leo

$$
\begin{aligned}
& \mathrm{v}_{\text {earth }}=30 \mathrm{~km} / \mathrm{s} \\
& \mathrm{v}_{\text {perihelion, transfer }}=\sqrt{\frac{G M s}{1.5 \times 10^{11}\left[\frac{2(4.286 / 1)}{1+(4.286 / 1)}\right]}}
\end{aligned}
$$




$$
\begin{aligned}
& =\sqrt{\frac{1.33 \times 10^{20}}{1.5 \times 10^{11}}\left[\frac{2 \times 4 / 286}{5.286}\right]} \\
& =37.92 \mathrm{~km} / \mathrm{s} \\
\text { So } \mathrm{v}_{\text {out hyper }} & =7.92 \mathrm{~km} / \mathrm{s} \\
\Delta \mathrm{v}_{\text {depart leo }} & =\sqrt{(11.2)^{2}+(7.92)^{2}}-8.0 \\
& =\sqrt{125.44+62.73}-8.0 \\
& =13.72-8.0 \\
& =5.7 \mathrm{~km} / \mathrm{s}
\end{aligned}
$$

The return requirement:

$$
\begin{aligned}
& \Delta \mathrm{v}_{\text {return }}=\mathrm{v}_{\text {aphelion, transfer }}-\mathrm{v}_{\text {aphelion, }} \mathrm{WH} \\
& \mathrm{V}_{\mathrm{Q}, \text { transfer }}=\sqrt{\frac{\mu}{Q_{t r}}\left[\frac{2}{1+\left(Q_{t r} / r_{\text {earth }}\right)}\right]} \\
& =\sqrt{\frac{1.33 \times 10^{20}}{4.286 \times 1.5 \times 10^{11}}\left[\frac{2}{1+4.286 / 1}\right]} \\
& =8.847 \mathrm{~km} / \mathrm{s} \\
& \mathrm{v}_{\mathrm{Q}, \mathrm{WH}}=\sqrt{\frac{1.33 \times 10^{20}}{4.286 \times 1.5 \times 10^{11}}(1-0.622)} \\
& =8.843 \mathrm{~km} / \mathrm{s} \\
& \therefore \Delta \mathrm{v}_{\text {departure, return }}=0.004 \mathrm{~km} / \mathrm{s}=4 \text { metres } / \mathrm{second} \text { !!! }
\end{aligned}
$$


This departure $\Delta v$ is TINY! - but the arrival $v \infty$ at earth orbit is approx $8 \mathrm{~km} / \mathrm{sec}$ !

\section{What about perihelion passage mining?}

This is not feasible because, although the Earth-departure $\Delta v$ will be very small, the deep space rendezvous $\Delta \mathrm{v}$ will be approx $8 \mathrm{~km} / \mathrm{s}$, and the departure $\Delta \mathrm{v}$ from the asteroid will also have to be approx $8 \mathrm{~km} / \mathrm{s}$. This assumes departure whilst still near perihelion, and this points to the requirement for a very short mining stay: (30-50 days max).

Therefore we assume an aphelion mining season, and as in the previous cases, assume a requirement for 1000 tonnes returned to Earth orbit, through a total $\Delta v$ of about $8 \mathrm{~km} / \mathrm{s}$.

We need to calculate the total amount of volatiles that is to be collected.

The rocket equation gives

$$
\begin{aligned}
& M_{\text {start }}=M_{\text {finish }} \times e^{\Delta v / v_{e}} \\
& \text { with } \Delta \mathrm{v}=8 \mathrm{~km} / \mathrm{s}, \quad \mathrm{v}_{\mathrm{e}}=3.1 \mathrm{~km} / \mathrm{s} \text {, and } ; M_{\text {finish }}=1000 \text { tonnes, } \\
& \text { then } M_{\text {start }}=13,000 \text { tonnes }
\end{aligned}
$$

(This is not a problem in terms of tankage fraction - ref Zuppero, Whitman and Sykes, 1993 - but is a problem in as much as it sets a very demanding throughput requirement on the extraction plant).

If 13,000 tonnes is to be collected in maximum of 50 days, then that is 250 tonnes/day, or approx $3 \mathrm{~kg} / \mathrm{sec}$.

Heat energy needed to volatilize $3 \mathrm{~kg} \mathrm{H}_{2} \mathrm{O}$ per second (from perhaps $-50 \mathrm{C}$ ) is

$$
\begin{array}{ll}
= & {[3000 \times 80+3000 \times 540+3000 \times 150] \mathrm{cal} / \mathrm{sec}} \\
= & (3000 \times 770 \times 4.2) \text { Watts } \\
= & 10 \mathrm{MW}
\end{array}
$$


A solar collector to provide $10 \mathrm{MW}$ is outside present technical capability.

To merely melt $\mathrm{H}_{2} \mathrm{O}$, however, would only require $-1.5 \mathrm{MW}$.

A 1 MW solar collector was envisaged in example \#1, processing regolith from $1989 \mathrm{ML}$.

What is required area? Solar insolation at $1 \mathrm{AU}=1.3 \mathrm{~kW} / \mathrm{m}^{2}$

So at $4 \mathrm{AU}$, solar insolation is about one-sixteenth, i.e., $80 \mathrm{~W} / \mathrm{m}^{2}$. Thus $1 \mathrm{MW}$ will require $12500 \mathrm{~m}^{2}$.

Thus, solar powered resource recovery from Wilson-Harrington appears impractical, with nuclear being the better (and longer-term) option. 


\subsection{Example \#6 : Comet du Toit-Hartley Mission}

Orbital parameters are:

Table 9.6 Orbital Parameters, p/DuToit-Hartley

\begin{tabular}{|c|c|c|c|c|}
\hline $\begin{array}{c}\text { Perihelion } \\
\mathrm{q}(\mathrm{AU})\end{array}$ & $\begin{array}{c}\text { Aphelion } \\
\mathrm{Q}(\mathrm{AU})\end{array}$ & $\begin{array}{c}\text { eccent } \\
\mathrm{e}\end{array}$ & $\begin{array}{c}\text { inclination } \\
\mathrm{i}(\mathrm{deg})\end{array}$ & $\begin{array}{c}\text { semi major axis } \\
\mathrm{a}(\mathrm{QU})\end{array}$ \\
\hline 1.201 & 4.814 & & 2.9 & 2.99 \\
\hline
\end{tabular}

Let us consider perihelion mining season:

Outbound velocity requirement:

$$
\begin{aligned}
& \mathrm{V}_{\text {Perihelion, transfer }}=\sqrt{\frac{1.33 \times 10^{20}}{1.5 \times 10^{11}}\left[\frac{2(1.2 / 1)}{1+(1.2 / 1)}\right]} \\
& =31.10 \mathrm{~km} / \mathrm{sec}
\end{aligned}
$$

$$
\text { So } \begin{aligned}
\Delta \mathrm{v}_{\text {depart leo }} & =\sqrt{11.2^{2}+1.1^{2}}-8.0 \\
& =\sqrt{126.65}-8.0=3.25 \mathrm{~km} / \mathrm{sec}
\end{aligned}
$$

Deep Space Rendezvous $\Delta \mathrm{v}$ Ds is given by

$\mathrm{V}$ perihelion, DT-H $-V_{\text {aphelion, transfer }}$

$$
\mathrm{V}_{\text {aphelion, transfer }}=\frac{31.10}{1.201}=25.895 \mathrm{~km} / \mathrm{sec}
$$




$$
\begin{aligned}
\mathrm{v}_{\text {peri, DT-H }} & =\sqrt{\frac{\mu}{q}\left(1+e_{d T H}\right)} \\
& =\left[e=\frac{Q-q}{Q-q}\right] \\
& =\frac{4.814-1.201}{4.814+1.201}=\frac{3.613}{6.015}=0.600 \\
\mathrm{v}_{\text {peri, DTH }} & =34.28 \mathrm{~km} / \mathrm{sec}
\end{aligned}
$$

Hence $\Delta \mathrm{v}_{\mathrm{DS}}=\mathrm{v}_{\mathrm{p}, \mathrm{dTH}}-\mathrm{v}_{\mathrm{a} \text {, transfer }}=8.39 \mathrm{~km} / \mathrm{sec}$

This $\Delta v$ is of course also what will be needed to leave du Toit-Hartley on Earth-return trajectory. This $\Delta \mathrm{v}$ Ds is actually less demanding than perihelion departure from WilsonHarrington, because the perihelion is further out.

The Rocket Equation says this implies 15,000 tonnes start mass of volatiles to return 1000 tonnes.

The mass processing requirement is affected by the fact that the solar constant at $1.2 \mathrm{AU}$ is only 0.7 of what it is at $1 \mathrm{AU}$.

NPV will be assisted by the short mission time (essentially 1 year) but negatively impacted by the very demanding mass processing rate and the very high velocity changes required, both out and back. 


\subsection{Example \#7 : p/Howell Mission}

Comet Howell has $\mathrm{q}=1.406$ and $\mathrm{Q}=4.882 \mathrm{AU}$

Due to its higher perihelion, it may be more favourable for a perihelion rendezvous than Wilson-Harrington or $\mathrm{p} / \mathrm{du}$ Toit-Hartley.

We calculate $\mathrm{v}$ outbound as before:

$$
\begin{aligned}
& \mathrm{V}_{\text {perihelion, transfer }}=\sqrt{\frac{\mu}{q_{t}\left[\frac{2\left(Q_{t} / q_{t}\right)}{1+\left(Q_{t} / q_{t}\right)}\right]}} \\
& =\sqrt{\frac{1.33 \times 10^{20} \times 2 \times 1.406}{1.5 \times 10^{11} \times 2.406}} \\
& =32.19 \mathrm{~km} / \mathrm{s} \\
& \mathrm{V}_{\text {Earth }} \quad=\quad 30 \mathrm{~km} / \mathrm{s} \\
& \text { so } \mathrm{V}_{\text {hyperbolic }}=2.19 \mathrm{~km} / \mathrm{sec} \\
& \text { and } \Delta \mathrm{v}_{\text {depart leo }}=\sqrt{125.44+4.796}-8.0 \\
& =3.41 \mathrm{~km} / \mathrm{s}
\end{aligned}
$$

Now we calculate $\Delta \mathrm{v}$ Ds (Deep Space Rendezvous $\Delta \mathrm{v}$ )

$$
\begin{aligned}
& \mathrm{V}_{\text {aphelion, transfer }}=\frac{32.19}{1.406}=22.89 \mathrm{~km} / \mathrm{s} \\
& \mathrm{v}_{\text {perihelion, Howell }}=\sqrt{\frac{\mu}{q_{H}}\left(1-e_{H}\right)}
\end{aligned}
$$




$\mathrm{e}_{\text {Howell }}=\frac{4.882-1.406}{4.882+1.406}=\frac{3.476}{6.288}=0.553$

$$
\begin{aligned}
\text { thus } \mathrm{v}_{\text {peri, Howell }} & =\sqrt{\frac{1.33 \times 10^{20}}{1.406 \times 1.5 \times 10^{11}}(1.553)} \\
& =31.29 \mathrm{~km} / \mathrm{s} \\
\text { so } \Delta \mathrm{v}_{\mathrm{DS}} & =31.29-22.86=8.43 \mathrm{~km} / \mathrm{sec}
\end{aligned}
$$

This will also be the departure $\Delta \mathrm{v}$ at the comet, at its perihelion, for Earth-transfer orbit injection into return trajectory. 


\subsection{Example \#8: Hypothetical Arjuna:}

(emphasising near-circular-orbit target, extended mining season, and near-continuous thrusting on return trajectory.)

Let us consider a hypothetical low-e Amor or Arjuna, with orbital parameters:

$\mathrm{q}=1.1 \mathrm{AU} \quad \mathrm{Q}=1.4 \mathrm{AU} \quad \mathrm{e}=0.12 \quad \mathrm{a}=1.25 \mathrm{AU} \quad \mathrm{i}=4 \mathrm{degs}$

$\mathrm{T}=(1.25)^{1.5}=1.4 \mathrm{yrs}$

Assume (say) 4 months outbound ballistic trajectory, with delta-v (deep space) supplied by chemical propulsion (because it has $\mathrm{I}_{\text {sp }}$ equal to steam rocket and is of adequate thrust). Launch is at ascending or descending node.

Assume (say) 9 months mining season centred about descending or ascending node. Time since launch will at end of mining season be about 13 months, and the asteroid will be some 90 degrees past descending /ascending. node.

Depart asteroid at this point for a $180 \mathrm{deg}$ continuous thrust arc to return to Earth.

Total project duration is then approx 22 months.

All configurations seem to suggest less than or equal to 180 degrees return thrusting arc, centred on a node; and therefore less than or equal to 180 degrees mining season, centred on a node; and 90 to 180 degrees of outbound trajectory.

Note that thrusting duration and mining / processing duration are both now about 200 days; it is sensible to optimise these so as to require the same power input. 


\section{Delta-v requirement:}

Inclination change requirement will dominate, at $0.5 \mathrm{~km} / \mathrm{s}$ for each degree. For $4 \mathrm{deg}, \Delta \mathrm{v}$ $=2 \mathrm{~km} / \mathrm{s}$. From previously worked examples, ellipticity-matching $\Delta \mathrm{v}$ requirement may be another $1.5 \mathrm{~km} / \mathrm{s}$. We will assume $3.5 \mathrm{~km} / \mathrm{s}$.

If using long-period thrusting, we need to increase $\Delta v$ capability of the propulsion system by factor of 1.5 to take into account the inefficiencies of non-instantaneous impulses (e.g. plane change not instantaneous at DN/AN). So a reasonable $\Delta v$ requirement is 5.25 $\mathrm{km} / \mathrm{s}$.

The implied thrust requirement, to return 1000 tonnes of payload through this velocity interval in 200 days, is about 500 Newtons. The propellant useage will be about 5400 tonnes.

The implied mining rate (assuming 10\% recovery of volatile from regolith) is 200 tonnes per day (or 8 tonnes per hour) suggesting a mining and processing equipment mass of 1 (one) tonne, from Chapter 6.

\subsection{Conclusions: Summary of results of Example Calculations:}

Arjunas are extremely accessible.

All return transfer orbits with aphelia less than 1.25 AU deliver the payload to Earth with hyperbolic velocity less than $1.4 \mathrm{~km} / \mathrm{s}$ and hence are amenable to capture by lunar flyby.

Atens are open to both perihelion and aphelion rendezvous missions.

Comet resource return missions with aphelion mining phase suffer very long mission times and inadequate solar power. Comet missions with perihelion mining phase need 
very large velocity changes to rendezvous and depart the object. NPV in these cases depend critically on the mass throughput ratio assumptions that one makes.

Mass-throughput requirements for positive NPV appear to be easily achieveable for the Arjunas and low-eccentricity Apollos and Amors.

The ice-drilling scenario appears very achieveable, provided the target bodies can be successfully identified. 


\section{Chapter 10: General Conclusions and Further Work.}

\subsection{General Findings}

The findings of this study are as follows:

1. Some Near-Earth Asteroids offer very promising targets as future orebodies for inspace activities, for reasons of accessibility, ease of return, apparent variety of source materials, and probable ease of extraction of both metals and volatiles, both of which are likely to be in heavy demand during the development of large-scale space infrastructure.

Such space resources will have to compete against Earth-launched resources. This may be made possible by applying the concepts of in-situ propellant production.

There has been a need expressed in the literature for a general methodology for determining the economics and feasibility of any proposed asteroid or comet mining project. This work addresses that need.

2. Asteroid geology is based on spectroscopic and photometric data for individual bodies, reflecting surface mineralogy and "weathering", and on inferred parallels with meteorites.

Asteroid - comet genesis of target body determines whether regolith-reclaim or drill-and-melt is to be the recovery process of choice.

A matrix of mineralogy / product / process choices was developed.

3. Near-Earth Asteroid geography is characterized by orbit location and type. The discovery rate of NEAs is now quite high, and there are an increasing number of "likely" targets being identified. 
A major problem is that only a small proportion of NEAs have been spectrally classified, hence their surface composition is not known. Major work is needed in order to define the mineralogically acceptable 'short-list'.

4. Target accessibility depends on velocity change $\Delta v$ to inject into transfer orbit, plus the velocity change needed to rendezvous with the target. These values are complex time-varying functions, and the present thesis did not propose to address this in detail; nevertheless, "global minima" can be estimated, by several methods. When serious work begins on asteroid mining projects, actual date-specific mission velocity requirements will have to be calculated, for the various project alternatives.

Ease of return depends on the asteroid departure delta-v, and on the hyperbolic velocity at Earth-return. Propulsive capture will be expensive inasmuch as it consumes otherwise-saleable returned volatiles. Lunar flyby gravity capture has been suggested as a way to remove hyperbolic velocity, although it will place a time constraint on the return dates. Aerobraking is another alternative. Further work is needed in 'capture technology'.

5. Considerations of mission profiles suggests a classification into five types:

- $\quad$ high-e, aphelion mining season (“Apollo-type")

- "Aten-type"

- $\quad$ spiral low thrust (low-e Amor or "Arjuna type")

- $\quad$ high inclination, low eccentricity

- $\quad$ high-e, perihelion mining season (“Comet-type")

In general, return missions to a particular body are not apparently advantageous, c.f. a new target.

Further work is needed on the mathematics of non-Hohmann (spiral) returns. 
6. Mining and processing methods can be readily conceptualised. However, there are many areas requiring study: anchoring into regolith on a body which has milli-g gravity; collection and handling material in milli-g gravity; minimum temperature and most rapid heat pulse for adequate volatiles release; system integration and minimum mass for required throughput.

Control via teleoperation and trained machine intelligence will require successful developments in neural net and fuzzy logic machine learning.

7. Propulsion and power options tend to focus on solar-thermal systems for the initial projects. Ultra-lightweight solar collector technology already exists. System integration has not yet even commenced but should be a straightforward engineering task.

8. Project economics is driven by the mission velocity requirements, by the propulsion system characteristics (particularly Isp), and by project time duration and timecost-of-money.

A cost delivered into leo of $\$ 200 / \mathrm{kg}$ or so will be essential for space raw materials resources recovery to be viable in the first few decades of the next century.

A "spider diagram" has been developed which clearly shows the inter-relationship of all relevant variables. This, together with the formulation of project Net Present Value in astronautical and celestial mechanics variables, enables a pro-forma, or 'roadmap' approach to project feasibility determination.

9. Reviews of examples, done on 'general principles' basis, give encouragement that cases do indeed exist that would prove economically feasible and make a profit whilst delivering resources to in-orbit purchasers. 


\subsection{Further Work:Identified Information and Technology Gaps}

Tasks which have been identified in this thesis, which can be carried out at low cost, e.g. as Honours and Masters degrees projects, are:

(i) thermogravimetric quantitative studies on volatiles release, using asteroidal analogues (gypsum, clay, calcite, oil shale) and ultimately using carbonaceous chondrite material; to determine yield -temperature-time curves.

(ii) development of specific target and mission alternatives, using Hohmann trajectories, via SAIC's Trajectory Optimizer program

(iii) Investigate the mathematics of non-Hohmann transfers.

(iv) pursue the spectral characterization of the Arjunas, as the most likely early targets.

(v) pursue neural net and fuzzy logic approaches to 'training' remotely operated machines to operate autonomously.

(vi) commence work on the conceptual mining and processing flowsheets discussed above.

In conclusion, this work provides a rigorous approach for performing Feasibility Studies on asteroid and comet mining ventures, and in addition shows how NPV can be used as a 'design-driver' and reality check in project concept selection and development. This work has identified the information gaps which need to be addressed in order to bring this concept into the realm of the immediately achievable. 


\section{Appendix 1 : Legal Regime for Asteroid Mining Tenure}

\section{Economic -Political -Legal Setting}

Clearly, at some time in the next 20 years or so, there will arise serious moves to commence mining activities in space, almost certainly on the Moon, and perhaps on the moons of Mars, and/or various asteroids.

The rules for governing such activities should be developed now, so as to ensure both encouragement of Humanity's peaceful expansion into Space, and also to ensure a clear contractual statement of mutual rights and responsibilities of the space resources enterprise vis a vis the international community, ie for the mining enterprise, clarity of title.

These rules will almost certainly draw from various aspects of national mining codes, from concepts found in the international law of the sea, and from both formal and customary law applying to such activities as deepsea fishing, research in international waters, and Antarctic research. Also relevant is international law pertaining to offshore oil platforms.

The situation of increasing activities in space, by various, sometimes competing, sometimes co-operating, national, international, and corporate organizations, represents a market for raw and processed materials, and hence peculiar opportunities and risks, for a would-be space resources developer.

Although there is a developing body of "Space Law", there does not exist -yet- a mechanism for purchasing, establishing precedence, or lodging a claim over, an area of, say, the Moon, or over an asteroid. Indeed, at the moment, space is a legal interregnum; a "terra nullius", a land belonging to no-one. How would one protect against appropriation of one's physical assets (i.e., piracy)? Would there be royalties to pay, and if so, to whom? The U.N. treaty on the peaceful uses of outer space needs to 
be considered together with international customary law deriving from maritime and aviation practices. The international law applying to oil platforms beyond the 12-mile limit, to deep ocean fisheries, to possible future deep ocean mining, and to research bases on Arctic Ocean ice floes, and to Antarctica, would all be relevant.

There are environmental concerns which need to be addressed namely assurance that returning payloads do not impact Earth, or if they do, that they will disintegrate safely at altitude high enough that there will be no harm.

The considerable velocity changes required, and launch window constraints, suggest that physical intervention is virtually impossible, so neither piracy nor physical policing are at all likely..

Management flexibility of private corporations vis-a-vis governmental bodies, together with freedom from governmental budgetary process and policy variations, and less constrained terms of reference, suggest significant competitive advantage for the independent profit-oriented organization.

\section{Legal regime for tenure of mining right.}

The following is a review of papers by Jasentuliyana, Harrison Schmitt, Zubrin, and others with a view to indicating the likely final approach to international recognition of asteroid mining. There are parallels with the Law of the Sea, and the recent "Boat Paper" modifying the resources regime of the Law of the Sea is an important signal as to the possible future legal regime.

The initial L.O.S. requirements and proposals regarding deep seabed mining in international waters had been perceived by many in the developed nations (those which had had consortia investigating exploitation of deep seabed resources) and by their mining industry, as inimical to free market commercial enterprises. 
The initial rules had been set up explicitly to transfer wealth to developing and underdeveloped countries, in pursuit of the "common heritage of mankind" principle, via a quasi-monopolistic "Enterprise", the operating arm of the mooted International Seabed Authority, which was to be the regulating agency.

According to Reynolds, 1981, the 1980 draft text of Part XI of the LOS set out "in considerable detail the powers and functions of a supra-national mining authority which was unprecedented in its nature and implications. The mineral resources of the seabed beyond national jurisdiction are seen by the international community, as represented by the UN General Assembly, as the "common heritage of mankind". It is intended that they should be exploited in a way which provides net revenue to be shared principally amongst developing nations as a mechanism of income transfer from "north to south". The original concept whereby an international Enterprise would be the sole exploiter of these resources was abandoned in earlier stages of the LOS conference and superseded by the so-called "parallel system", whereby private or national entities and the Enterprise would share the development of the resources...."

This "parallel system" draft then proposed a series of requirements on prospective operating entities which were perceived to be commercially crippling:

- the Enterprise would be funded by States Parties to set up a minesite

- the Enterprise would freely appropriate its chosen $50 \%$ of any Area explored by any prospector or operator

- it would have access to finance from States Parties on interest free or concessional terms

- $\quad$ operating entities would be obligated to make technology available to the Enterprise on "fair and reasonable" terms

- $\quad$ entities would pay royalties to and share net revenues with the Authority

The requirement to cede $50 \%$ of every discovery to the Enterprise, together with the demand for technology transfer, and the essentially free funding, caused a rebellion among the then-existing private enterprise consortia. It was also noted, (Reynolds, 
1982) that the following could be non-commercial motivations for a State to become active in deep seabed mining:

- for national prestige

- $\quad$ to capture resources lacking within its territorial area

- $\quad$ to access strategic metals

- $\quad$ to pre-empt access to an area by a rival State

- $\quad$ to establish a presence in an area

All of these reasons were seen as threatening the free marketeers, because they were prompts for state subsidization.

This relationship between the Seabed Authority and the Enterprise, and its proposed subsidies and special advantages, were seen by the free market miners as providing such an overwhelming commercial advantage to the Enterprise that they successfully lobbied their home nations to repudiate and refuse to sign the final L.O.S. Treaty, and instead mutually agreed to come to alternative arrangements, including unilateral national legislation, mutually recognised. This group comprised US, UK, Belgium, Italy, and West Germany. Over the dozen years since the L.O.S. Treaty was opened for signature, these and other nations have refrained from signing, and commercialization has failed to eventuate. Some time ago, the Secretary- General of the UN, perceiving that the unacceptability of the resources regime section, Section XI, was stalling international ratification of the Treaty, most of which was regarding such things as navigation, fisheries, the law on piracy, etc., and noting "the prolonged delay in commercial deep seabed mining", instigated circuit-breaker talks to renew efforts for consensus. These resulted in the "Boat Paper".

The "Boat Paper", 1994, is an annex to the L.O.S. (officially the "Draft Resolution and Draft Agreement relating to Implementation of Part XI of the UN Convention on the Law of the Sea") and modifies Part XI of LOS as follows:

- costs of the International Seabed Authority to be paid by the UN until commercial activities commence, thereafter to be met from assessed contributions by the member states (with restrictions on bureaucracy and budget)

- initially, the role of the Enterprise will be restricted to monitoring 
- $\quad$ states no longer obligated to fund a minesite for the Enterprise

- a contractor who has contributed an Area to the Authority shall have after 15 yrs, first right of refusal

- $\quad$ there shall be no compulsory transfer of technology

- approvals of Plans of Work for exploration and for exploitation shall be procedurally clear and open to audit, and shall provide security of tenure under the conditions of the contract

- the Enterprise is constrained to follow commercial principles, and is prohibited from subsidization and dumping

This revised approach has won the acceptance of the US and other key States Parties, and resulted in the signing of the Treaty by the requisite 60 nations and its entry into operation on 16th November 1994 as international law.

The significance of this recent ratification of the LOS is that it is a step towards defining an acceptable regime for international regulation of mining activities in extraterritorial areas. The parties have been forced to take into consideration the commercial realities of requirement for security of tenure over a granted area, and enforceability of contracts, as a right of the operating entity; and the entity's right to proprietary control over its technology and data. The adjustments made in the Boat Paper could probably still be improved on, and the requirement that $50 \%$ of explored area, as chosen by the Enterprise, be ceded to the Enterprise, is still widely regarded as confiscatory. However, the LOS is clearly a precedent, albeit an imperfect one, for any regime that would be set up internationally to regulate asteroid mining.

It is possible of course to "go it alone", both in deep seabed mining and in asteroid mining. Regarding LOS, the US had clearly considered this path, going so far as to pass unilateral enabling legislation. But sooner or later, a corporation has to deliver the product to a market, and it would not want to have to worry about an embargo. 
Jasentuliyana, the Director of the UN Office for Outer Space Affairs, has taken particular interest in the possible legal regimes and commercial structures for asteroid mining, and lists for comparison (Jasentuliyana, 1990):

- $\quad$ LOS deep seabed mining (position pre-Boat Paper)

- the Convention on the Regulation of Antarctic Mineral Resource Activities

- $\quad$ several successful international space operations activities and organizations, viz Intelsat and Inmarsat, and the international cooperation on the Giotto Mission.

In this review, Jasentuliyana hints that an "Intelsat-like" structure could most easily address the apparent legal impediments of the "common heritage" concept.

Harrison Schmitt proposed a similar Intelsat-like structure for "Interlune", his concept of an international consortium for mining the Moon for Helium-3. (Schmitt, 1992). In the above reference, he notes that Intelsat as a user-based and managed organization has developed because of a coincidence of available technology and obvious international need...and that it was an example of international cooperation not only technically successful but also utilitarian and profitable (returning some $15 \%$ p.a. to its investor states).

According to Schmitt, the Interlune concept was specifically designed to address the intents of the Outer Space Treaty and the Moon Agreement, as a monopoly international organization governed by representatives of national, user, and investor parties. However, in conversation, Schmitt now downplays the likelihood of Interlune succeeding in its original form, and suggests "a private initiative may now be more appropriate" (Schmitt, pers. comm, 1995).

\section{Legal Basis of Claims}

The various approaches to exploitation of terrestrial ore bodies are worth looking at: by the process of claim, or by application for mining lease, land which is held to be public domain, or belonging to the Crown, or the "res publica", i.e., "common heritage" property, is made available, on payment of royalties and on acceptance of 
jurisdiction and perhaps special operating conditions, for exploitation by the claimant / lease-holder / concession-holder. Note however that there are cases of miners rejecting or disputing the claim of the Crown to have jurisdiction, especially when the Crown is perceived to have done nothing to assist in either the development, or the adequate and fair administration of the resource. An Australian example is the Eureka Stockade rebellion of the goldminers of Ballarat, Victoria, in 1854.

Different nations have a variety of approaches to the allocation of mining claims, leases, or tenements, and a variety of approaches to taxation, e.g. by royalty, fees, etc.. Operations can work claims directly as a contractor to the crown or republic, e.g., on a cost-plus basis, or on purely commercial terms as an assigned operator (or concessionholder).

Marks, 1993, reviews the legal precedents available and arrives at a "consensual" model, drawing on the self-regulatory regimes set up by the miners of the Californian goldrush, who were in a legal inter-regnum, being theoretically trespassers on US government lands, but in fact beyond the reach of US effective law. "..The most workable near-term system should probably not be rooted in new and prospective lawmaking, but in....negotiated rule making created by the miners themselves." These rules, once tested and settled, then subsequently become the basis for formal codification.

"Pending development of an explicit tenure and title system, the better model is one of consensual regulation by the miners themselves..." Marks then lists the basic rules the '49ers' of the Californian goldfields evolved: (i) claim ownership based on priority of possession; and (ii) the right to hold and work the claim based on actual possession and proper marking of boundaries.

In all of the above, the ownership of the land was not deemed to have passed from the state, but only the priority to work the deposit under the rules of the state. 
Marks suggests that "priority of possession" in space mining might be derived from "tele-robotic possession", and notes that this concept, i.e., occupation via an active robotic agent, has gained some legal standing in at least one court battle over the rights to recovered sunken treasure.

Marks further notes in passing, "compare the contemplated situation in space: no sovereign effectively able or entitled to exercise jurisdiction or practical control over the miners". This situation may be good because it creates the necessary opportunity to devise a similar miner-to-miner (self-regulatory) system, before "rampant legislating begins".

In "Access to a Res Publica Internationalis: the case of the Geostationary Orbit", Weissner (1986?), in discussing GEO 'slots', a limited resource, describes the Justinian Code of ancient Rome, which is relevant to interpretation of the term "common heritage of mankind". The Justinian Code classified things into res in patrimonio, things under exclusive individual control, res publica, the common property of the state, e.g., roads, viaducts, ports..., res communis, that open to everyone, and res nullius, things owned by no-one (and hence subject to appropriation by occupation and use). He identifies the case of GEO slots as equivalent to res publica and, in passing, identifies the high seas as res communis. He also considers, and dismisses, the idea of an International Orbit Authority, in analogy with the LOS's International Seabed Authority.

McCandless \& Garver (1989) identify "res communis" as identical with the "common heritage of mankind" concept. They also propose, as a regime that would fulfil quite clearly the "common heritage" principles, a Lunar Resources Authority, the purpose of which would be to regulate resources utilization on the Moon.

In contrast, Goldman (1984) reiterates the US treaty negotiators' assertions that "use" of space resources is specifically allowed by the Outer Space Treaty, and that this therefore includes the right to extract resources. This is interpreted to be in conformity with the "common heritage" provisions by virtue of claiming that whilst resources "in 
place" are the property of humanity at large, resources recovered or reclaimed become the property of the miner, by the allocation of his labour and skills; if this interpretation is to be meaningful, the developer must surrender some freedoms, e.g. (i) accept jurisdiction; (ii) make available some part of the product on fair market terms.

Dunstan, 1987, says: "It is predicted .... (that) the self-interests of space faring nations, as well as the beginning of private uses of outer space, will result in a continued shift from regulation of space activities by international treaty to regulation by private contract and bilateral agreement, but that eventually, international mechanisms for dispute resolution will be required ..."

\section{Possible UN Office of Outer Space Affairs approach:}

An approach that the UN Office of Outer Space Affairs could take on the allocation and regulation of rights to recovery of extraterrestrial resources is as follows.

1. It is accepted that the expansion of human activity into outer space is desirable, for reasons of

(i) humanity's long-term supply of energy and materials for a prosperous and healthy life for all (e.g., energy from Satellite Solar Power Stations or from Helium-3).

(ii) philosophically, the establishment of an open frontier in space replaces the zero-sum game competition over boundaries and resources on Earth.

(iii) humanity's long-term immunity from any planet threatening catastrophe, e.g., comet impact-induced "nuclear winter".

(iv) philosophically, the urge to spread life throughout the solar system, to continue the creative work of God.

These aims will be met when large space cities are developed, such as those described by O'Neill and earlier by Dandridge Cole.

2. It is generally agreed that the capital for such major activities can only come from commercial entities, which survive by delivering valuable goods or services 
at an acceptable price to willing corporate or governmental purchasers. The primary reason why commercial enterprises represent the only viable source of capital is because financial disciplines and social responsibilities now preclude governments from major projects.

3. It is now accepted that at least conceptually, there are several "space resource recovery" possibilities:

- lunar or Uranian Helium-3 for fusion power

- $\quad$ possibly water ice from permanently shadowed craters at the lunar South Pole

- $\quad \mathrm{NiFe}$ metal from certain asteroids, with Platinum Group Metals as a byproduct

- $\quad$ volatiles from certain asteroids and short-period comets

- $\quad$ water ice from depth below the regoliths of Phobos or Deimos

4. The recovery of such material would provide a "social good" inasmuch as it would leverage and advance humanity's capability to expand into outer space and ultimately colonise it, and thus is to be supported.

5. In order to provide for the encouragement of commercial entities' investment of capital, it is accepted that a mechanism must be put in place by which "rights", "claims", or "leases" over non-terrestrial removable resources can be created, universally recognised, regulated, worked, and traded, with certainty of title.

6. Such a right, claim, lease, or title is taken to represent a contract between the recipient commercial entity intending to recover and use or sell the resource material, and the regulatory agency, acting on behalf of humanity infurtherance of the goals mentioned above in (1), with the sanction of the UN and the agreement of the spacefaring nations, as the only parties capable of intervening.

7. The contract might agree, for example, the following: 
- that the regulatory agency will, on the appropriate authority, universally recognised, grant to the entity (with reasonable attached conditions) a right to mine a defined property.

- that such a created right becomes a valuable property in and of itself.

- that materials recovered from such a lease or claim become, upon collection or separation from the regolith or matrix of the body to which the lease applies, the legal property of the operating entity, and as such can be legally bought and sold.

- in return for the grant of these potential commercial benefits, society requires general recognition of the created titles.

- the entity must "work" the lease or claim within a reasonable predetermined timeframe, or forfeit the lease (e.g., detailed astronomical studies to commence within one year, launch of either prospector probe or resource recovery operation within six years).

- the entity must supply information of the following type in support of applications for leases: identity of body, orbital elements, nonbinding letters of intent to purchase products from prospective customers or of intent to supply finance from partners investors or bankers.

- the issue of leases should be on a "first come, first served" basis, so as to avoid "second-guessing" the ability of small applicants to put together the required technical and financial capability, and to avoid the major operators tying up all prospective bodies.

- leases may be "bid for" by entity making an offer of royalty rate, such royalty to be paid by delivery into regulatory agency ownership in earthorbit of the agreed percentage of product.

- the entity must make some set minimum percentage of its production available on the open market (so as to fulfill the "common good" test).

- the life of the lease should be long enough to encourage permanent occupation, either human or tele-presence, and to enable later sale of a lease over a body which has already proven profitable, and should be renewable on terms favourable to the initial venturer. 
- claims for bodies of major dimensions less than (say) $25 \mathrm{~km}$ diameter will only be issued to cover the entire body. This is necessary so as to avoid unpoliceable boundary disputes. Claims over larger bodies will be issued to cover areas of maximum extent of (say) 1500 square $\mathrm{km}$.

- the regulator will have responsibility to review the Earth-orbit-capture mechanism proposed and to set minimum safety parameters for control of risk of impact.

- it must be recognised that non-regulated mining can not be readily banned, because physical policing at the resource site will be almost impossible, due to delta- $\mathrm{v}$ and synodic phasing considerations (except for the case of the Moon). Thus the regime for regulation must be such that the benefits of compliance must be seen to outweigh the possible advantages of noncompliance, for the venturer. 


\section{Appendix 2: Economics of Launcher Systems}

The present cost of launch to LEO is approx $\$ 10,000$ to $\$ 15,000$ per kilogram (Stuart \& Gleave, 1991).

By far the cheapest launcher at the moment is the Russian Proton, quoted to cost $\$ 60$ million, with a payload into LEO of a little less than 20 tonnes; this is roughly $\$ 3000$ per kilogram.

The probable launch costs of new small expendable launchers was reviewed by NASA and they found that "dedicated small launcher costs show no signs of dropping below the $\$ 5,000,000$ per launch threshold considered vital for energetic growth in the small commercial payload market" (quoted in Ad Astra, Jan 1993, p 16). The Orbital Sciences Corp Pegasus launcher costs $\$ 30,000 / \mathrm{kg}$.

The only possible viable expendables are those exhibiting extreme simplicity, such as Hudson's "Liberty" (no longer being actively advanced), simple solids or hybrids, which might deliver under $\$ 1,000 / \mathrm{kg}$ in quantity production, and an upgrade of the Proton.

Many writers have identified that reusable launchers, operated with airline-like maintenance regimes, flight frequencies, and ground support, will be necessary to bring about major cost reduction.

The extent of cost reduction achievable with total reuseability is addressed by Griffin and Claybaugh in JBIS 47 pp119-122 in which they produce a general parametric cost model. See below for details of this model. For a vehicle such as Hotol, at a flight frequency of 20 per year, the model predicts $\$ 600 / \mathrm{kg}$. For a less pessimistic flight frequency, of 140 per year, equating roughly to a payload of 1000 tonnes to orbit per year, the cost will obviously be lower. 
However, Parkinson, in Spaceflight 32, pp 248-249, and $\underline{36}$, pp 400-403, looks at total system lifetime cost for Hotol, and reports that the cost including system development is approx $\$ 5,000 / \mathrm{kg}$, and that the recurring (operating) cost is approx $\$ 1,400 / \mathrm{kg}$, both assuming 20 flights per year and system lifetime of 20 years. This flight frequency however is certainly below that which would represent either full utilization of the vehicle or a breakout into space indusrtialization, and would appear very pessimistic for a totally reusable vehicle, given that extra flights will spread the development cost thinner.

What is the Demand Elasticity? -i.e., to what extent will the market demand for launch services expand in response to a reduction in "airfreight" charges? We do not know. It may be that mobile phone systems may provide the market to expand from 20 to 40 or more flights per year.

We do have good indications however, that the threshold for space tourism is at about $\$ 500 / \mathrm{kg}$, giving a ticket price to LEO of $\$ 50,000$. This has been found in various market surveys, and in addition, the market becomes very large as the price falls below $\$ 50,000$ per ticket (P. Collins pers comm).

\section{Launcher Cost Model}

A cost model is presented in Griffin and Claybaugh, which can be used to predict lower-bound space launch costs:

\section{Cost Model}

Total cost of launch $=$ cost of expended hardware + cost of propellant + cost of launch site operations incl refurbishment

$$
\mathrm{C}_{\mathrm{T}}=\mathrm{C}_{\mathrm{H}}+\mathrm{C}_{\mathrm{P}}+\mathrm{C}_{\mathrm{O}}
$$


cost per $\mathrm{kg}$ is $\mathrm{c}_{\mathrm{T}}=\frac{C_{H}+C_{P}+C_{O}}{M_{P L}}$

\begin{tabular}{|c|c|c|c|}
\hline $\mathrm{C}_{\mathrm{H}}$ & $=$ & $\begin{array}{l}\mathrm{c}_{\mathrm{H}} \mathrm{mf} \mathrm{M}_{\mathrm{S}} \\
\mathrm{c}_{\mathrm{H}} \operatorname{mf} \mathrm{R} \mathrm{M}_{\mathrm{PL}}\end{array}$ & $\begin{array}{l}\text { where } \mathrm{mf} \text { is mass fraction of vehicle } \\
\text { expended and } \mathrm{Ms} \text { is vehicle structural mass } \\
\text { (including avionics), and where } R=\frac{M_{S}}{M_{P L}}\end{array}$ \\
\hline $\mathrm{C}_{\mathrm{P}}$ & $=$ & $c_{P} M_{P}=c_{P} P M_{P L}$ & where $P=\frac{M_{P}}{M_{P L}}$ \\
\hline $\mathrm{C}_{\mathrm{O}}$ & $=$ & $c_{L} L R M_{P L}$ & $\begin{array}{l}\text { where } c_{L}=\text { hourly cost of labour hours per } \\
\text { flight per kg dry mass of vehicle }\end{array}$ \\
\hline
\end{tabular}

(and $R=\frac{M_{S}}{M_{P L}}$ (as before))

Thus $\quad \mathrm{c}_{\mathrm{T}}=\frac{C_{H}+C_{P}+C_{O}}{M_{P L}}$

now becomes

$c_{T}=c_{H} m f R+c_{P} P+c_{L} L R \quad$ where $m f$ is fraction of hardware expended;

$$
R=\frac{M_{S}}{M_{P L}} \quad ; \quad P=\frac{M_{P}}{M_{P L}} \quad ; \quad \mathrm{L}=\text { labour hours/flight } / \mathrm{kg}
$$

Note that amortization of vehicle fleet can be treated by putting $\mathrm{mf}<1$ for reusable vehicles, to take into account their replacement. 
"it should be noted in passing that a vehicle that can be considered fully reusable in a practical sense ... must be regarded as being expended in fractional increments over its operational lifetime. Also, preservation of a fleet of vehicles must include some accounting for the cost of unreliability and subsequent replacement of a lost vehicle. Those effects can be included within the operations cost category, or by adjusting the expendable fraction, mf"

For expendables, $\mathrm{mf}=1$; $\mathrm{P}$ ranges from $\sim 30$ (for the Atlas rocket) to 50 for Delta and Titan IV; and $\mathrm{R}$ ranges from $\sim 2$ to $\sim 6$.

Other data quoted by Griffin and Claybaugh are:

$$
\begin{array}{llll}
c_{\mathrm{H}} & - & \text { rockets } & -\$ 20000 / \mathrm{kg} \\
& & \text { aircraft } & -\$ 1000-2000 / \mathrm{kg} \\
c_{\mathrm{P}}= & \$ 2 / \mathrm{kg} \text { for Lox } / \mathrm{LH}_{2} ; \$ 6 / \mathrm{kg} \text { for storables } \\
c_{\mathrm{L}}= & \$ 60 / \mathrm{hr}
\end{array}
$$

This model was shown to correspond well with the known launch costs of present expendables.

Labour hours for the X-15 program (which was not optimized to minimize labour time requirement), was $\sim 1.6$ hours/flight $/ \mathrm{kg}$ mass. Flight rate was $20 / \mathrm{yr}$; for $20 \mathrm{yrs,}$ with 3 vehicles. (Note: it is unclear whether maintenance cost of B-52 launch planes was taken into account.)

If similar labour efficiency could be attained with a reusable orbiter, the operations cost would be $\sim \$ 600 / \mathrm{kg}$.

The authors then conclude that total cost including propellant has a lower bound of $\sim \$ 300 / \mathrm{lb}$ or $\$ 800 / \mathrm{kg}$.

However, if we use their same logic, and put in quoted values for Hotol (from RC Parkinson, Spaceflight, $\underline{32}$ : 248-249, and $\underline{36}: 400-403$ ), we get: 
$c_{T}=c_{H} \operatorname{mf} R+c_{P} P+c_{L} L R$

$$
\begin{aligned}
& \mathrm{P} \text { for Hotol }=\frac{210}{7}=30 \\
& \mathrm{R} \text { for Hotol }=\frac{32}{7}=4.5
\end{aligned}
$$

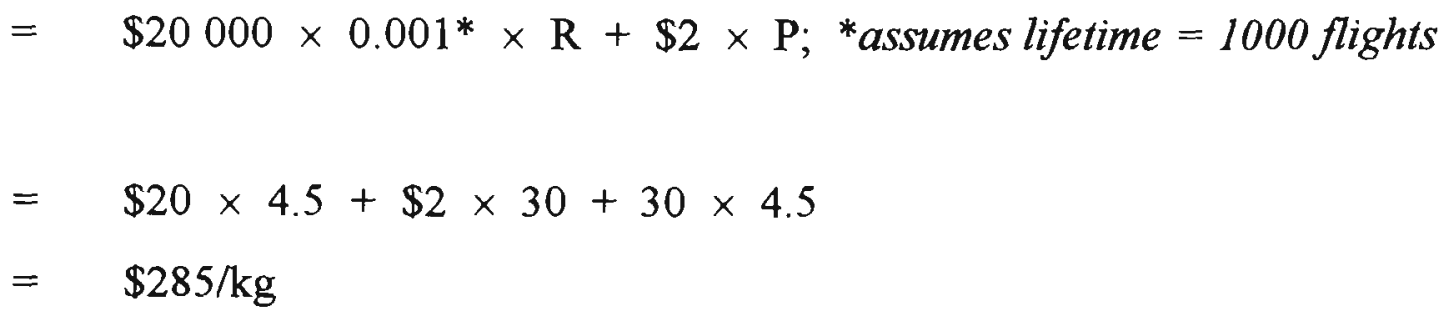

The hardware cost issue remains unclear here, but will be low, for an assumed lifecycle of 1000 flights, because at a specific hardware cost of $\$ 20000 / \mathrm{kg}$ (the cost of the shuttle), the hardware cost component.

$$
\begin{aligned}
& =\quad \mathrm{c}_{\mathrm{H}} \mathrm{mf} \mathrm{R}, \text { is } \\
& =\quad \$ 20000 \times 0.001 \times \mathrm{R}(\text { say 4.5) }=\$ 90 / \mathrm{kg}
\end{aligned}
$$

This suggests a probable lower bound total cost of approx $\$ 380 / \mathrm{kg}$. 


\section{$\underline{\text { References and Bibliography }}$}

Agosto, W., (1981), Beneficiation and Powder Metallurgical Processing of Lunar Soil Metal, Space Manufacturing 4, Proceedings of 5th Princeton AIAA Conference.

Ahrens, I. J., \& Harris, A. W., (1992), Deflection and fragmentation of Near-Earth Asteroids, Nature $\underline{360} 3$ December 1992 p429-433.

Anon (1994), Inco boosts automation, Mining Journal, London 27 May 1994.

Asphaug \& Benz (1995), The Tidal Evolution of Strengthless Planetesimals, Lunar \& Planetary Sciences Conference 26, pp55-6, March 1995.

Atzei, A., et al, (1991), ESA Preparatory Program for the Rosetta / CNSR Mission, Space Technology $11 \mathrm{pp} 1-15,1991$.

Baiden, G. R., \& Henderson, E., (1994), LHD teleoperation and guidance proven productivity improvement tools, CIM Bulletin 87 No 984 October 1994 p47-51.

Baiden, G. R., Flewelling, S., \& Scoble, M., (1993), Robotic systems development for mining automation, CIM Bulletin 86 No 972 June/August 1993 p75-77.

Bell, J. F., (1995), Q-Class Asteroids and Ordinary Chondrites, 26th Lunar and Planetary Sciences Conference, March 1995.

Belton, M. J., \& Chapman, C. R., (1994), First Images of Asteroid 243 Ida, Science $\underline{265}$ September 1994 p1543-1547.

Belton, M. J., Veverka, J., et al, (1992), Galileo Encounter with 951 Gaspra: First pictures of an Asteroid, Science 25718 September 1992 p1647-1653. 
Bilder, R. B., Cameron, E. N., Kulcinski, G. L., \& Schmidt, H. H., (1989), Legal Regimes for the Mining of Helium-3 from the Moon, University of Wisconsin, Wisconsin Center for Space Automation and Robotics, 1989. WCSAR - TR - AR3 - 8901-1.

Binzel, R., Gehrels, T., \& Matthews, eds (1989), Asteroids II, University of Arizona Press, 1989.

Britt, D. T., Kring, D. A., \& Bell, J. F., (1995), The Density/Porosity of Asteroids, 26th Lunar and Planetary Sciences Conference, March 1995.

Burbine, T. H., \& Binzel, R. P., (1993), Asteroid Spectroscopy and Mineralogy, in Asteroids, Comets, Meteors,1993, pp255-270; publ IAU.

Burrows, R. R., (1985), Example Solar Electric Propulsion System Asteroid Tours using Variational Calculus, J. Spacecraft 22 No 3 May/June 1985 p325-331.

Carter, R. A., (1993), New Technology makes mining remotely possible, EMJ Engineering and Mining Journal September 1993 p18-21.

Cheng, A., (1995), NEAR: First Launch of the Discovery Program, Lunar \& Planetary Sciences Conference 26, March 1995.

Chapman C (1995), NEAR: Eros as the key to S-type Conundrum, Lunar \& Planetary Sciences Conference 26, March 1995.

Chapman C R (1994), "Dazzling Demise of a Comet” (SL-9), Nature 37028 July 1994 pp245-246

Chapman C R , Morrison D (1994), “Impacts on Earth by asteroids and comets: Assessing the hazard", Nature $\underline{367} 6$ January 1994 p33-39 
Clark, Fanale, and Salisbury (1992), "Meteorite - Asteroid Spectral Comparison - the Effects of Comminution, Melting, and Recrystallization”, Icarus, 97, pp288-297, 1992.

Collins P, et al. (1994), "Commercial Implications of Market Research on Space Tourism", 19th International Symposium on Space Technology and Science, Yokohama, May, 1994.

Collins P \& Isozaki K, (1995), "JRS Research Activities for Space Tourism", 6th International Space Conference of Pacific Basin Societies.

Connelly H B, et al (1994), "Carbon and the formation of reduced chrondrules", Nature $\underline{371} 8$ September 1994 pp136-139

Cordell B M, \& Wagner S L (1986), Strategies, Synergisms, and Systems for the Manned Exploration of Mars, IAF Congress, paper IAF-86-320, 1986

Council on Ocean Law, Washington DC, (1994), "Summary of the April 15, 1994 Boat Paper" and "April 15 Draft Resolution and Draft agreement Relating to the Implementation of Part X1 of the 1982 UN Convention on the L O S.", in Oceans Policy News the Newsletter of International Oceans Law, 11 No. 2, May 1994.

Cox and Cole, (1964), The Challenge of the Planetoids, publ Chilton Books, 1964

Cronin et al, (1988), "Organic Matter in Carbonaceous Chondrites", in "Meteorites in the Early Solar System", eds Kerridge and Matthews, U of Arizona Press 1988

Cutler A \& Hughes M, (1985), Transportation Economics of Extraterrestial Resource Utilization, AIAA/SSI 1985

Cutler, A H, (1987), "Accessibility of Near-Earth Asteroids for Resource Exploitation", in Space Manufacturing 6, the Proceedings of the 8th Princeton / AIAA / SSI Conference, 1987, 
Davis D R, Friedlander A L, \& Jones T, (1993), "Role OF Near-Earth Astroids Mission for the Space Exploration Inititive", in The Resources of Near-Earth Space, ed J S Lewis et al, Univ of Arizona Press, 1993

Deininger W D \& Vondra R J (1991), Spacecraft and Mission Design for the SP-100 Flight Experiment, Journal of the British Interplanetary Society 44 pp217-228, 1991.

Dermott S F et al (1994), "A circumsolar ring of asteroidal dust in resonant lock with the Earth", Nature 36930 June 1994 pp719-723

Dewar J A (1994), Atomic Energy: the Rosetta Stone of Spaceflight, Journal of the British Interplanetary Society 47 pp199-206, 1994.

Dula, A (1979), Free Enterprise and the Proposed Moon Treaty, L-5 News, Nov 1979.

Dunbar R S (1979), "The Search for Asteroids in the L4 and L5 Libration Points in the Earth- Sun System", 4th Princeton / AIAA Conference on Space Manufacturing Facilities, 1979; AIAA-79-1437

Fanale \& Salvail, (1990), "Evolution of the Water Regime of Phobos", Icarus, $\underline{88}$ pp380$395,1990$.

Farinella P et al (1994), "Asteroids falling into the Sun”, Nature $\underline{371} 22$ September 1994 p314-317.

Fearn, D G, (1982), Electric propulsion of Spacecraft, JBIS $\underline{35}$, pp156-166, 1982.

Froning Jr H D, Czysz P A (1993), "Impact of Emerging Technologies on Manned Transportation between Earth and Space", Space Technology 13 No.3 p285-290 1993.

Gaffey M J (1989), Asteroid Surface Mineralogy, in Asteroids II, ed Binzel, Gehrels, and Matthews, Univ of Arizona Press, 1989. 
Gehrels T, ed., (1994), Hazards due to Comets and Asteroids, Univ of Arizona Press, 1994

Gehrels, T (ed.)(1979), Asteroids, University of Arizona Press, 1979

Gertsch R (1984), "Asteroid Mining" and "A Baseline Lunar Mine", in Space Resources, NASA Publ SP-509, eds McKay, McKay, \& Duke, 1992 (proceedings of NASA-ASEE Summer Study Workshop at California Space Institute, 1984)

Glaser, P E, (1982), "Solar Power Satellites", in Space Industrialization, CRC Press, 1982

Ganguly, J \& Saxena, S (1989), Theoretical Predictions of Volatile Bearing Phases and Volatile Resources in Some Carbonaceous Chondrites, Space Manufacturing 7, AIAA/SSI, 1989.

Gaffey M J \& McCord T B (1976), "Asteroids: A Source of Natural Resources for Terrestrial and Extra-Terrestrial Applications", Lunar Utilization Conference, 1976

Gaffey M J \& McCord T B (1982), Mineralogical and Petrological Characterizations of Asteroid Surface Materials, in Asteroids, ed T Gehrels, University of Arizona Press, 1982

Goldman, N, Space Law and Space Resources, AIAA / SSI Space Manufacturing Conference.

Golubeva L F, Shestopalov D I (1992), "S-Asteroids of the Main Belt and Meteorite Sources", (translated from) AstronomicheskiiVestrik 26 No.6 p116-124 November / December 1992 p602-608

Grady M, Wright I P (1994), "Martians come out of the closet", Nature $\underline{369} 2$ June 1994 p356 
Grallert H K H, Berry W, "Progress in ESA Winged Launches. Configuration Studies", Space Technology 13 No.1

Grossman and Williams (1990), 'Inflatable Concentrators for Solar Propulsion and Dynamic Space Power", Jnl Solar Energy Engineering, Nov 1990, 112, pp229-236. (reports the work done at L'Garde Inc, Tustin, Calif).

Griffin and Claybaugh, The Cost of Access to Space, JBIS 47 pp119-122

Haslett, R, (1993?) Space Nuclear Thermal Propulsion Program, Trans. Amer. Nuclear Soc. 70 p $122-3$

Hartmann, Tholen, and Cruickshank, "The Relationship of Active Comets, "Extinct" Comets, and Dark Asteroids," Icarus 69 pp33-50

Hartmann W K (1982), Mines in the sky promise riches, a greener Earth, Smithsonian, $\underline{13}$ no. 6 , Sept 1982 pp70-77

Helin E, \& Shoemaker, E (1978), Earth Approaching Asteroids as Targets for Exploration", in NASA Conference Publication 2053, Asteroids: an Exploration Assessment, ed Morrison and Wells, 1978

Henbest N (1993), "The "planet" that came in from the cold", New Scientist 14 November 1993 p46

Herrick S (1979), Exploration and 1994 Exploitation ofGeographos, in Asteroids, ed. T Gehrels, University of Arizona Press, 1979.

Hoashi M , Brooks RR, Reeves RD (1993), "Palladium, Platinum and Ruthenium in iron meterites and their taxonomic sigificance", Chemical Geology 1061993 p207-218 
Hoffman M, Debhan H, Neukum G, Geyer E H (1993), "Photographic Observations of Four Near-Earth Asteroids", Acta Astronomica 43 1993 p61-67

Howe S D, Metzger J D, “Space Nuclear Propulsion”, Trans Amer Nuclear Society Isozaki et al (1994), "Considerations on Vehicle Design Criteria for Space Tourism", IAF 1994

Jasentuliyana, N (1990), International Space Law and Cooperation and the Mining of Asteroids, Annals of Air and Space Law, 15 pp343-355, 1990.

Jones TD, Lebofsky LA, \& Lewis JS, (1990), The Composition and Origin of the C, P, \& D Asteroids:Water as a Tracer of Thermal Evolution in the Outer Belt, Icarus $\underline{88}$, pp172-192 1990

Kargel J S (1994), "Metalliferous asteroids as potential sources of precious metals", Journal of Geophysical Research 9925 Oct 1994 No E10 p21129-21141

Kelley, Boain \& Yen, Robotic Planetary Science Missions with Nuclear Electric Propulsion, JPL tech paper, JPL, 4800 Oak Grove Drv, Pasadena, California 91109

Kerr R A (1994), "Bareing the Secrets of Asteroid Ida", Science 26625 Sept 1994 pp 1322

Kerridge J F, (1985), Carbon, Hydrogen, and Nitrogen in Carbonaceous Chondrites: Abundances and Isotopic Compositions in Bulk Samples, Geochim. Cosmochim Acta 49: $1707-1714,1985$.

Kerridge J F and Matthews M S, eds., (1988), Meteorites and the Early Solar System, University of Arizona Press 
Kloski, L (1995), Screw Plate technology for anchoring structures on the Moon, International Symposium on Mine Mechanisation and Automation, June 1995, Colorado School of Mines

Kuck D L (1979), Near-Earth Extraterrestrial Resources, in Space Manufacturing 3, Proceedings of the 4th Princeton/AIAA Conference, May 14-17, 1979.

Kuck, D L (1981), Economic and Political Climate for Exploration of Space Riches, Commercial Operations in Space 1980-2000, 18th Goddard Memorial Symposium 51 Science and Technology Series, Amer Astronaut Soc 1981.

Kuck, D (1995), “The Exploitation of Space Oases”, in Princeton Conference on Space Manufacturing, May 1995

Kuck, D L (1992), In-Situ Recovery of Water from Dormant Comet Cores and Carbonaceous Chondrites, Space ‘92

Lagerkvist C L \& Barucci A (1992), "Asteroids: Distinctions, Morphologies, Origins and Evolution", Surveys in Geophysics $\underline{13}$ : pp 165-258, 1992

Langevin Y (1995), The Rosetta Mission to Primitive Bodies of the Solar System, Lunar \& Planetary Sciences Conference 26, March 1995.

Lau and Hulkower, (1985), "On the Accessibility of Near-Earth Asteroids", AAS-AIAA Astrodynamics Conference, 1985, paper AAS 85-352

Leonard R, Blacic J, \& Vaniman D: (1987), The Economics of Mining the Martian Moons, AIAA, 1987

Levy et al., (1973), "The Organic Analysis of the Murchison Meteorite", Geochim. Cosmochim. Acta, 37: pp475-483, 1973. 
Lewis J S , Hutson M L (1993), “Asteroidal Resource Opportunities Suggested by Meteorite Data", in Resources of Near-Earth Space p523-542, ed Mattews, Galtieri and Lewis, University of Arizona, 1993

Lewis J S , Jones T D , Farrand W A (1988), "Carbonyl Extraction of Lunar and Asteroidal Metals", in Engineering, Construction and Operations in Space p111-122, ed Johnston and Metzel, American Society of Civil Engineers 1988

Lewis J S, McKay D S, Clark B C (1993), "Using Resources from Near-Earth Space", in Resources of Near-Earth Space, ed Lewiw,Matthews \& Guerrieri, University of Arizona Press, 1993

Lewis J S, McFadden L A, Swindle T D (1991), "Near-Earth Asteroids and the History of Planetary Formation", Eos Transactions American Geophysical Union $\underline{72}$ No 44, 29 October 1991 p473-480

Lewis J S, Nozette S (1983), Extraction and Purification of Iron-Group and Precious Metals from Asteroidal Feedstocks, AAS 83-236, in Space Manufacturing Conference, 1983, publ. Amer Astronautical Society, $\underline{53}$ pp351-353

Lewis J S (1994), "Plantinum Apples of the Asteroids", Nature 3728 December 1994 p499-500

Lewis J S (1991), "Non-Terrestrial Resources of Economic Importance to Earth", IAF Montreal 1991

Lewis J S (1993), "Logistical implications of water extraction from near-Earth asteroids", AIAA / SS1 Space Manufacturing Conference 1993

Lewis J S (1991a), "Construction Materials for an SPS Constellation in Highly Eccentric Earth Orbit", Space Power 10 No 3 and 41991 p353-362 
Lewis, J S \& Lewis, R A, (1987), "Space Resources: Breaking the Bonds of Earth", Columbia Univ Press, 1987

Lewis, J S, Ramohalli, K, \& Triffet, T, (1990), Extraterrestrial Resource Utilization for Economy in Space Missions, IAF 1990.

Luke R (1993), "Computerized vehicle dispatching goes underground”, CIM Bulletin $\underline{86}$ No 967 February 1993 p49-53

Macllwain C \&Verrall M (1994), "Comet collision boosts controversy over Global Protection Strategy", Nature $\underline{370} 21$ July 1994 pp165

McCandless B S \& Garver L (1989), "Political, Economic, and Legal Considerations in International Cooperation on a Lunar Base", AIAA, 1989.

McKinnon \& Benner (1995), "Constraining the density of Comets from Crater Chains on Callisto and Ganymede", Lunar \& Planetary Sciences Conference 26, March 1995 p945

Marks B S \& Walker M A, (1993), Law and Policy Consideration for Space Mining in the 21 st Century, AIAA / SS1, 1993

Meinel C P \& Parks K (1985), "Stratagies to minimise Costs of Extraterrestrial Resources", IEEE 1985 EASCON 2213-7/85000-111 pp111-127

Melosh H J (1994), “The Geology of Multi-Ring Impact Basins, the Moon and other Planets", Nature $\underline{368} 3$ March 1994 p24

Morrison D (1993), “An International Program to Protect the Earth from Impact Catastrophe: Initial Steps", Acta Astronautica 30 p 11-16 1993

Morrison D \& Niehoff J (1979), Future Exploration of the Asteroids, in Asteroids, ed. T. Gehrels, University of Arizona Press, 1979 
Nagatomo M (1996), An Approach to Develop Space Solar Power as a New Energy System for Developing Countries", Solar Energy ㅌ6, No 1, pp111-118, 1996.

Nakanishi S (1985), Experimental Performance of a 1 Kilowatt Arcjet Thruster, NASA Tech Memorandum 87131, 1985

Nantel J, Piche A, Scobie M (1988), "A Canadian program in mining automation", CIM Bulletin $\underline{81}$ No 914 June 1988 p36-40

Nieland V Y, Parkinson R C (1993), "The AN-225 / Interim Hotol Launch Vehicle", Space Technology 13 No.3, p 311-316 1993

Niehoff, J (1978), Asteroid Mission Alternatives, in NASA Conference Publication 2053, 1978: Asteroids: an Exploration Assessment, eds. Morrison \& Wells).

O'Leary, B (1982), “Asteroidal Resources for Space Manufacturing”, in Space Industrialization, O'Leary, ed., CRC Press, 1982.

O'Leary B (1977), "Mining the Apollo and Amor Asteroids", Science 19722 July 1977 p363-366

O’Neill G K (1974), Space Colonies, Physics Today, Sept 1974

Olsson-Steel D (1989), "Spotting the Earth Smashers!", Southern Astronomy September/October 1989 p22-23, and Southern Astronomy November/December 1990 p24-27

Olsson-Steel D (1987), "Theoretical meteor radiants of Earth approaching asteroids and comets", Australian Journal of Astronomy 2 April 1987 p21-35

Olsson-Steel D (1988), "Theroretical meteor radiants of recently discovered asteroids, comets, and twin showers of known meteroid streams", Australian Journal of Astronomy 2 April 1988 p93-101 
Olsson-Steel D (1988), "Identification of Meteroid Streams from Apollo Asteroids in the Adelaide Radar Orbit Surveys", Icarus $1988 \underline{75}$ p64-96

Ostro S B et al. (1991), "Asteroid 1988 DA: Radar Evidence for a Metallic Composition", Science 252 June 71991

Ostro et al, (1991), Asteroid 1986DA: Radar Evidence of a Metallic Composition, Nature, 7 June 1991 pp 1399-1404

Ostro et al., (1995), "Extreme Elongation of Asteroid 1620 Geographos from Radar Images", Nature 375 pp 474-477, June 1995

Oxnevad, Kurt (1991), An Investment Analysis Model for Space Mining Ventures", International Astronautical Federation Conference, 1991

Paige D A (1994), "Chance for snowballs in hell”, Nature 36919 May 1994 p356

Parkinson R, Costs of Reusable Spacecraft, Spaceflight 32, pp 248-249, and $\underline{36}$, pp 400403

Perry's Chemical Engineer's Handbook, McGraw Hill

Pietrass A E (1984), Trajectory Design for an Ion Drive Asteroid Rendezvous Mission Launched into an Ariane Geostationary Transfer Orbit, AIAA-AAS Astrodynamics conference August 1984, paper AIAA-84-2058.

Podnieks E R \& Siekmeier J A (1994), Role of Mining in Lunar Base Development, Journal of the British Interplanetary Society, 47, pp543-548, 1994

Prialnik D, \& Mekler Y, (1991), The Formation of an Ice Crust below the Dust Mantle of a Cometary Nucleus, Astrophys Journal 366, pp 318-323), 1991. 
Ramohalli, Kirsch and Priess, "Figure of Merit Approach to Extraterrestrial Resource Utilization”, J Propulsion $\underline{8}$ pp240-246

Reichhardt I (1994), "Congress directs NASA to plan an Asteroid hunt", Nature $\underline{370} 11$ August 1994 p 136-139

Reynolds, J (1981), Seabed Mineral Resources and the Law of the Sea Conference, J Australian Foreign Affairs Review, July 1981

Reynolds, J (1982), Deepsea Mining and the Regime of the New Convention, in Seminar on the Law of the Sea, Australian Branch of the International Law Association. June 19, 1982

Rivkin \& Lebovsky (1995), Three-Micron Spectra of Hydrated Asteroids, Lunar \& Planetary Sciences Conference 26, March 1995, pp 1173-4

Robert F, and Epstein S (1982), The Concentration and Isotopic Composition of Hydrogen, Carbon, and Nitrogen in Carbonaceous Meteorites, Geochim. Cosmochim Acta, $\underline{46}: 81-95,1982$.

Rosenberg S D (1991), “Concepts of Lunar Resource Utilization”, AIAH /SAE / ASME / ASEE 27th Joint Propulsion Conference - June 24-26 1991

Rudolph, D, \& King, D,(1984), Electrostatic Thrusters for Spacecraft Prime Propulsion, AIAA-84-1446, 20th Joint Propulsion Conference, 1984

Russell C et al (1995), The DIANA Discovery Mission: SEP Mission to the Moon and a Comet, Lunar \& Planetary Sciences Conference 26, March 1995

Sagan C, Ostro S J (1994), "Dangers of Asteroid Deflection", Nature 3687 April 1994 p501 
Scotti J V, Rabinowitz D L, Marsden B G (1991), "Near miss of the Earth by a small asteroid", Nature $\underline{354} 28$ November 1991 p287-289

Senay M C, Jewitt D (1994), "Coma formations driven by carbon-monoxide release from comet Schwassman-Wachmann", Nature 37115 September 1994 p229-231

Sharp, W, Miller, R, \& Gertsch, R (1993), In-Situ Processing of a Near-Earth Comet, in DOE Workshop: Space Energy Resources Utilization Initiative, March 1993, Lafayette, Louisiana

Shoji, J (1985), Solar Rocket Component Study, Tech Report AFRPL TR 84-057, US Air Force Rocket Propulsion Laboratory 1985.

Shultz N R, (1994), "Figures of Merit and Attributes for Space Fusion Propulsion", Fusion Technology, 25 pp182-197, March, 1994.

Society of Mining Engineers (1985), Mineral Processing Handbook, ed. Weiss, SME, NY 1985.

Solem, Johndale (1993), Medusa: Nuclear Explosive Propulsion for Interplanetary Travel, Journal of the British Interplanetary Society 46 pp21-26, 1993.

Space Research Associates (1986), "Report on Satellite Solar Power Systems", Space Power 6 pp1-98 1986, Pergamon Press

Steel D , McNaught R H (1991), "The Anglo-Australian Near-Earth Asteroid Survey", Australian Journal of Astronomy. 4(2), October 1991. pp 42-48

Steel D, Snow P (1991), “The Tapanui Regions of New Zealand: A “Tunguska” of 800 years ago?", Presented at ACM 91 Flagstaff Arizona 1995 p1-4

Steel D (1992), "Earth Impactors: Australian and International Search Programs", Geophysics Down Under June /December 1992 p31-34 
Steel D (1992), "The Search for Earth Threatening Asteroids", Proceedings of the XVth NACAA Adelaide 1992 pA1-A7

Steel D (1992), "The Search for Death from the Sky"

Search 23 No1 January/Feburary 1992 p25-27

Steel, Duncan (1995), Rogue Asteroids and Doomsday Comets, publ. John Wiley, 1995

Strangway D W (1979), "Moon and Asteroid mines will supply raw material for space exploration", Canadian Mining Journal May 1979 p44-47, 52

Stuart J R \& Gleave, J (1990), "Key Small Satellite Subsystem Developments, AIAA Space Programs and Technologies Conference, 1990, AIAA-09-3576

Stuart J R \& Gleave, J (1991), US and International ELVs, Rail/Gas Guns, and Launch Sites, Students for the Exploration and Development of Space Conference, Colorado School of Mines, Feb 1991.

Studier et al (1972), "Origin of Organic Matter in the Early Solar System”, Geochem et.Cosmochem Acta $\underline{36}$, pp 189-215, 1972

Sullivan et al, (1995), Geology of 243 Ida, 26th Lunar and Planetary Science Conference, 1995 pp 1375-76

Sviatoslavsky I N, Jacobs M (1988), "Mobile Helium-3 Mining and Extraction Systems and its Benefits Towards Lunar Base Self-Sufficiency", Space88: Engineering , Construction and Operations in Space, August 1988 p310-321.

Tholen D J \& Barucci A (1989), Asteroid Taxonomy, in Asteroids II, eds Binzel, Gehrels \& Matthews, University of Arizona Press, 1989.

Thomson, W T, (1986), Introduction to Space Dynamics, Dover, NY, 1986. 
Ulamec S (1995), Technical Challenge, Scientific Thrill: a Long-term Lander on an Active Comet, Lunar \& Planetary Sciences Conference 26, Houston, March 1995.

United Nations Office of Outer Space Affairs (1994), United Nations Treaties and Principles on Outer Space, Vienna, 1994.

US Army Corps of Engineers: (1992), Proceedings of a Workshop on Extraterrestrial Mining and Construction, 1990, USACERL Special Report M-92/14.

Veverka J (1995), Asteroid 433 Eros; High Resolution Imaging and Spectral Mapping from NEAR, Lunar \& Planetary Sciences Conference 26, March 1995.

Veverka J, Thomas P, Simonelli D, et al (1994), "Discovery of Grooves on Gaspra”, Icarus 1071994 p 72-83.

Vilas, F (1994), A Cheaper, Better, Faster Way to Detect Water of Hydration on Solar System Bodies, Icarus 111, pp456-467, 1994.

Wallace R, Blume W, Hulkower N \& Yen C L, Interplanetary Missions for the Late Twentieth Century, J Spacecraft 22 pp316-324.

Wang S \& Zhang H (1995), Mysterious Ice Blocks from the Sky”, Purple Mountain Observatory, Academica Sinica, Nanjing, P.R.China.

Weismann and Campins, (1993), Short Period Comets in Resources of Near Earth Space, ed Lewis et al, Univ of Arizona Press, 1993.

Weissner S, "Access to a Res Publica Internationalis: the case of the Geostationary Orbit”, St. Thomas University School of Law, Miami, Florida.

Weidenschilling S J (1994), "Origin of Cometary Nuclei as 'Rubble Piles", Nature $\underline{368}$ 21 April 1994 pp721-728. 
Wetherill G (1979), Apollo Objects, Scientific American, 1979.

Williams O, Jackson E, Montieth I (1992), "A Control System for Teleoperated and Automated Mining Equipment Exposed to Severe Moisture, Corrosion and Shock", 5th Canadian Symposium on Mining Automation Vancover 1992 p184-204.

Williams \&Tedesco (1995), Evidence of hydrated minerals in E and M type asteroids, 26th Lunar and Planetary Sciences Conference, Houston, March, 1995.

Yen C L (1984), Mission Opportunity Maps for Rendezvous with Earth-Crossing Asteroids, IAA-AIAA Astrodynamics conference 1984, paper AIAA-84-2029.

Zakaria S (1994), "Automatic Haulage Truck expert system”, CIM Bulletin 87 No 976 January 1994 p74-77

Zubrin R (1991), Nuclear Salt Water Rockets: High Thrust at 10,000 sec Isp., Journal of the British Interplanetary Society, 44 pp371-376, 1991.

Zuppero, Whitman, \& Sykes (1993), Report of the Space Energy Resource Utilization Initiative Workshop, March 1993, Univ of Southwestern Louisiana.

Zuppero, A (1996), Discovery of Abundant Accessible Hydrocarbons Nearly Everywhere in the Solar System, in Proceedings of the 5th International Conference on Engineering, Construction, and Operations in Space; Amer. Soc. Civil Engineers, Albuquerque, June 1996. 


\section{Internet Resources:}

European Asteroid Research Node

http://129.247.214.46/

Planetary Data System Small Bodies Node:

http://pdssbn.astro.umd.edu

Planetary Sciences at the NSSDC:

http://nssdc.gsfc.nasa.gov/planetary/planetary_home.html

Lunar \& Planetary Institute:

http://cass.jsc.nasa.gov/lpi.html

Asteroid \& Comet Impact Hazard:

http://ccf.arc.nasa.gov/sst/

Known Near-Earth Asteroids:

http://ccf.arc.nasa.gov/sst/table_list.html

IAU: Minor Planet Center:

http://cfa-www.harvard.edu/ graff/mpc.html

Icarus Subject Index:

http://astrosun.tn.cornell.edu/Icarus/indices/

Steward Observatory Asteroid Relational Database:

http://dorothy.as.arizona.edu:8008/soard/

Spaceguard Foundation:

http://www.mi.astro.it/SGF/

Ted Bowell's page, Lowell Observatory:

http://www.lowell.edu/users/elgb/Welcome.html

PDS Home Page:

http://pds.jpl.nasa.gov/ 


\section{Listings of Near Earth Asteroids:}

The following tables have been imported from "Unusual Objects", a site on the WorldWide Web, address http://cfa-www.harvard.edu/graff/lists/Unusual.html . This site is maintained by the Minor Planet Center at the Smithsonian Astrophysical Observatory (address http://cfa-www.harvard.edu/cfa/ps/mpc.html).

Another major source of asteroid data is SOARD, the Steward Observatory Asteroid Relational Database, at the University of Arizona. The particular usefulness of SOARD is that the remote user can go into the database and construct a file using his or her own selection criteria, and then export the file by ftp to the home computer. SOARD's address is http://dorothy.as.arizona.edu:8008/soard/

Lists of Near-Earth asteroids which have orbital plane inclinations less than 15 degrees have been created by the above method, and are also given below, these being the NEAs with least plane change $\Delta \mathrm{v}$ cost. The high-accessibility target bodies will be a subset within this group.

Estimates of minimum mission velocity are calculated via spreadsheet, for these listed targets, using the formulae of Shoemaker and Helin. 


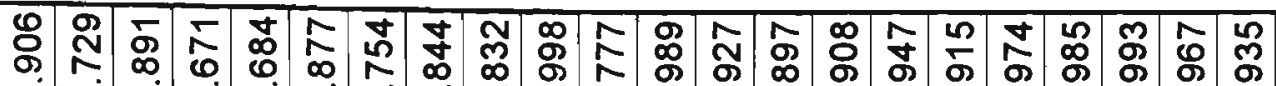

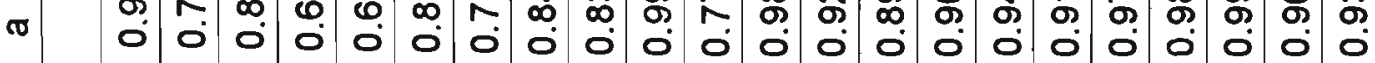

ஜ

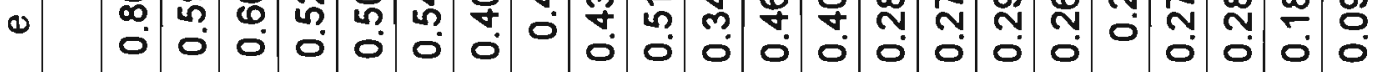

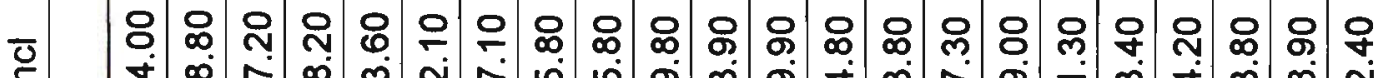

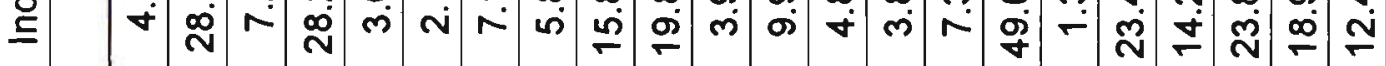

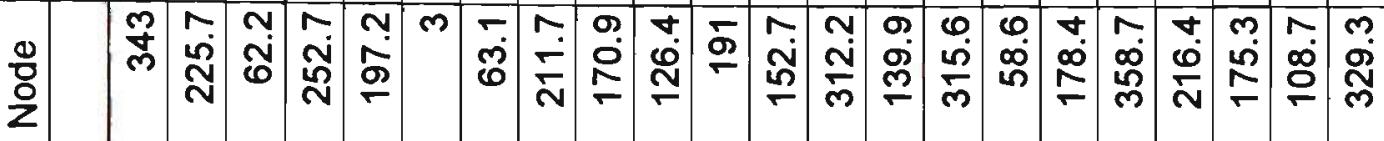

-

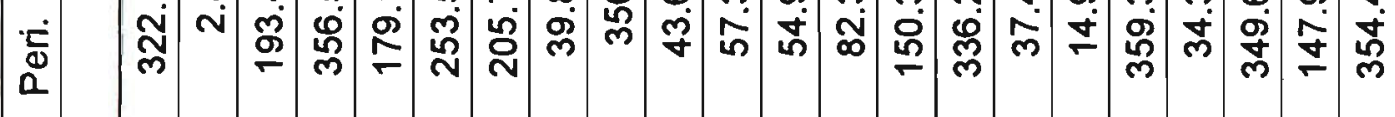

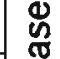

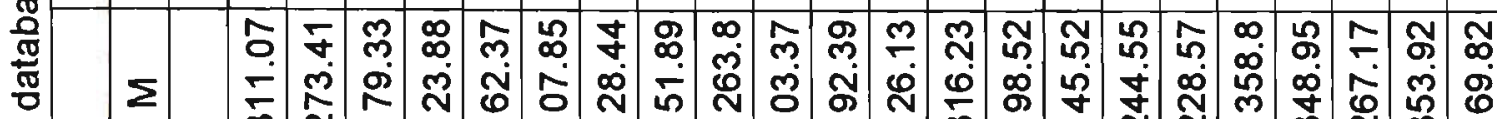

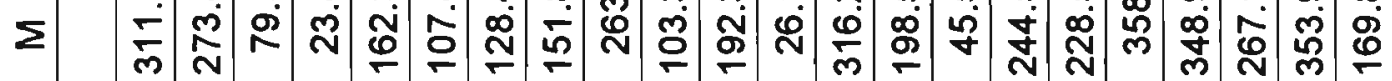

$\stackrel{\grave{\Xi}}{ \pm}$

灾

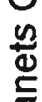

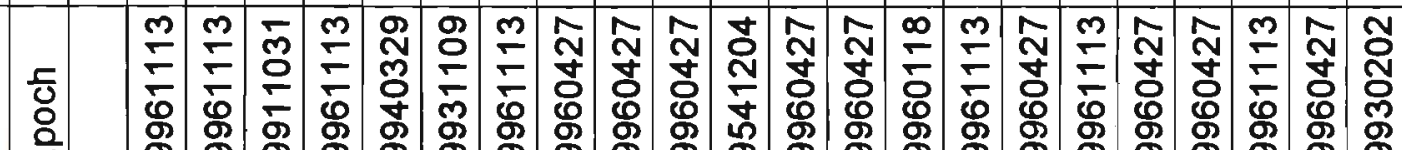

ш

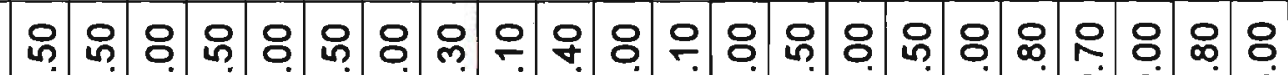

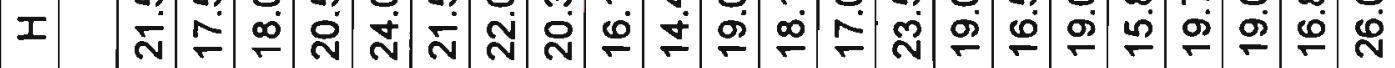

要

要

సิ

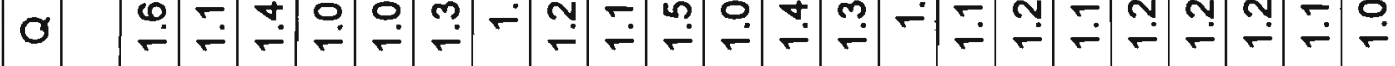

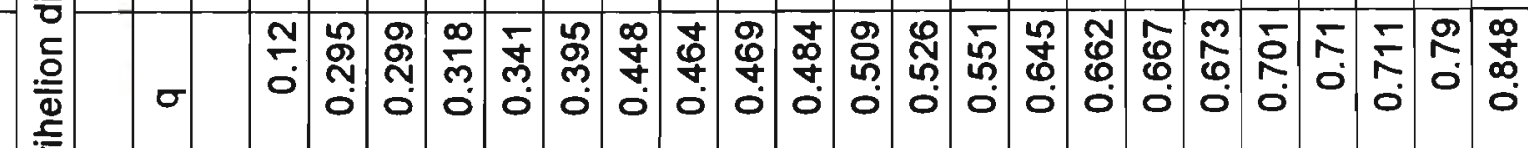

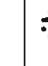

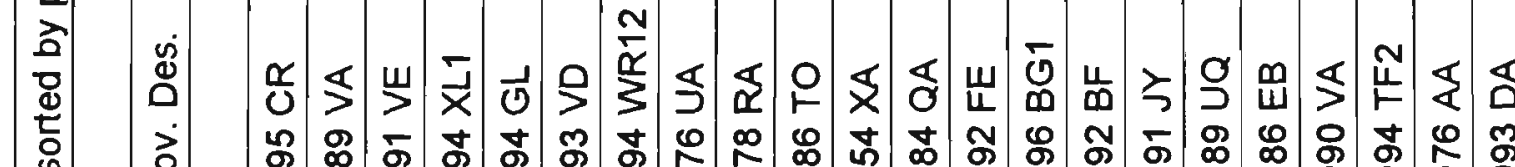

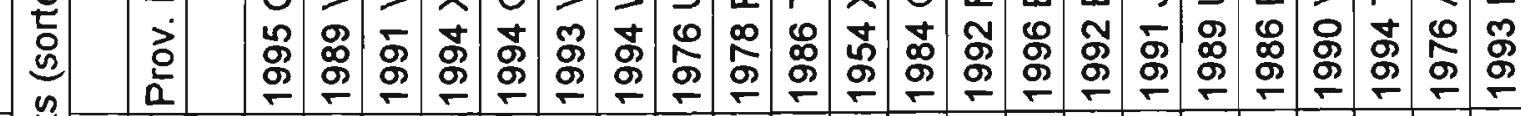

\%

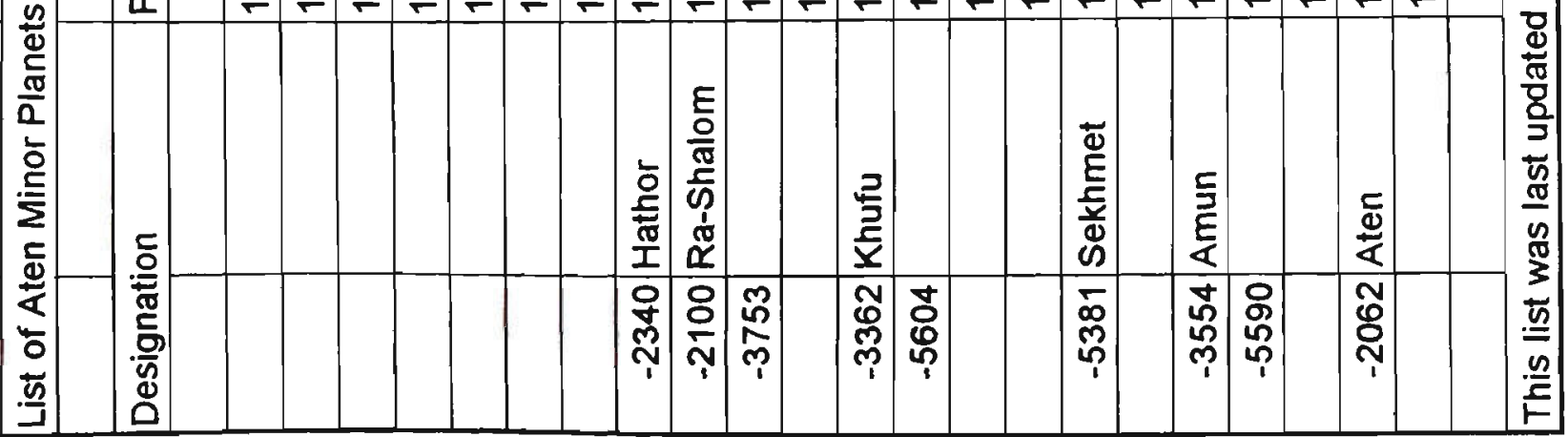




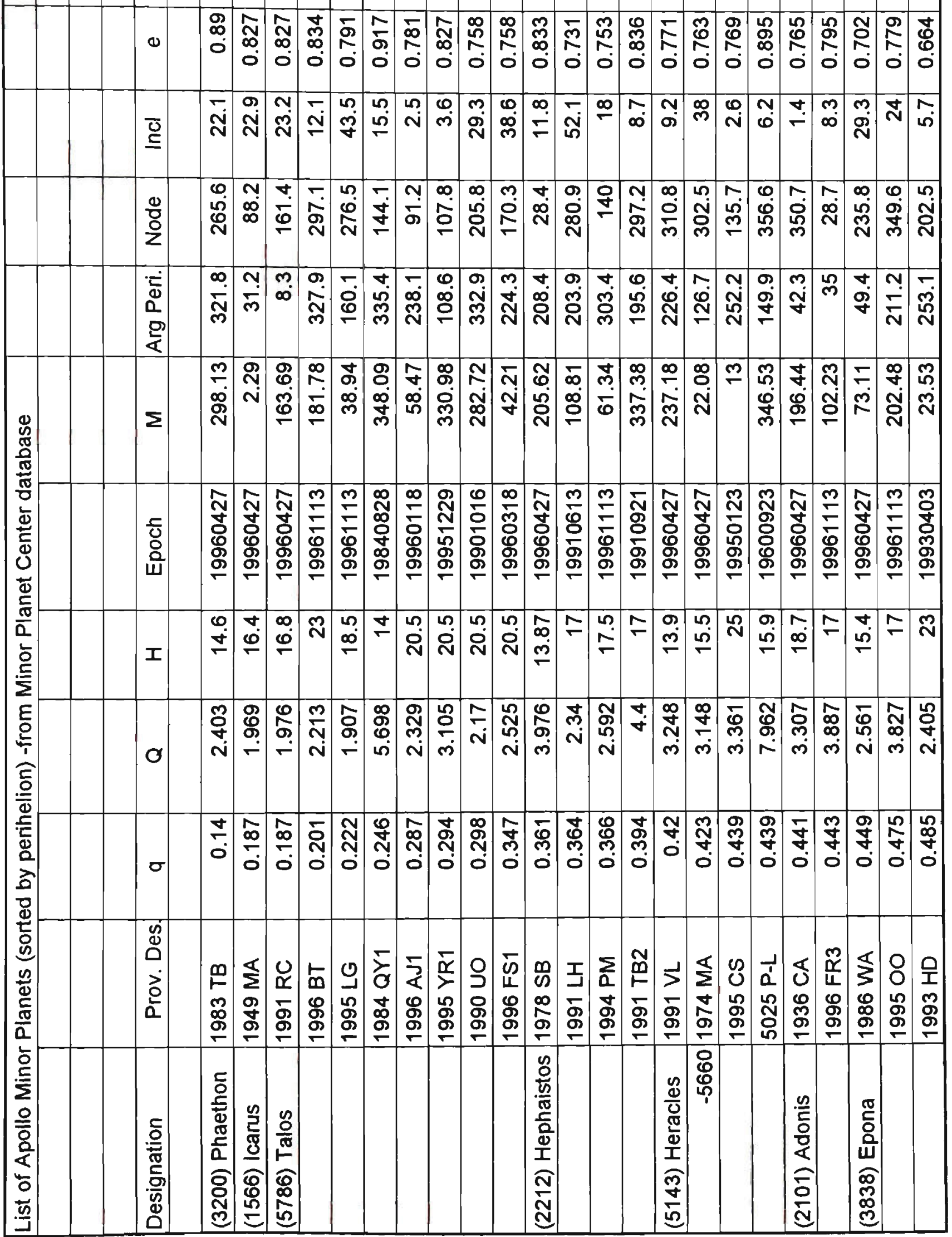




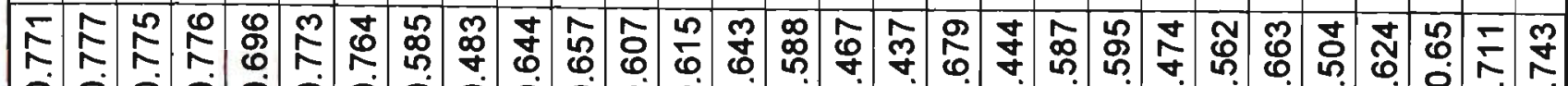

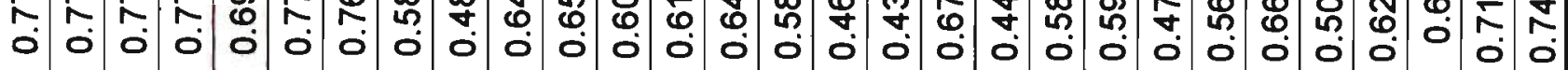
๓

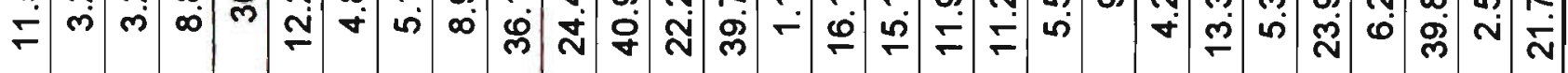

$\tau^{-}$

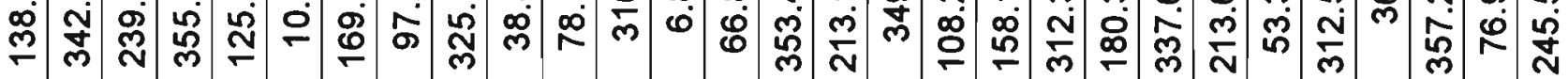

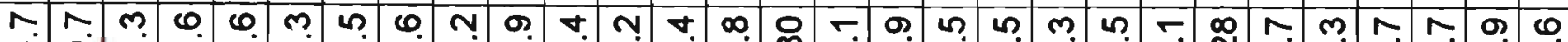
응 穴

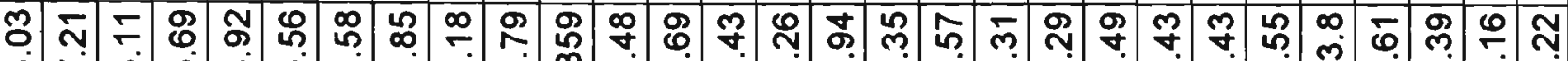
œ

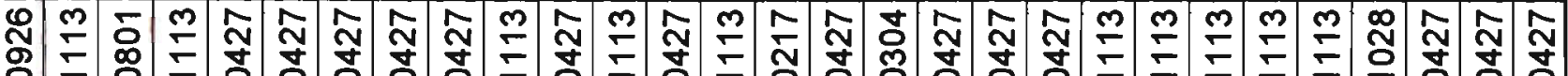
ర్

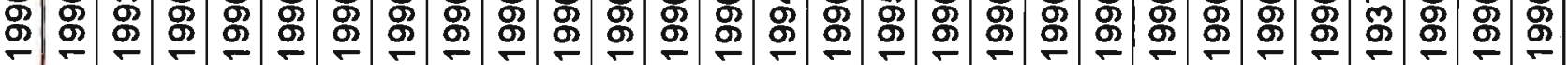

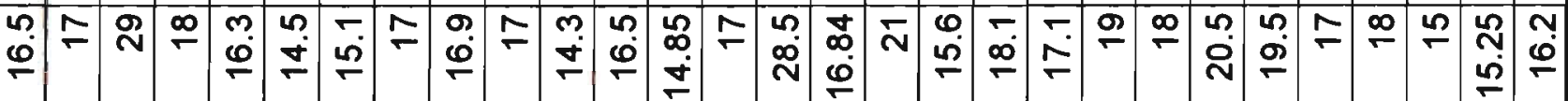

ఏ

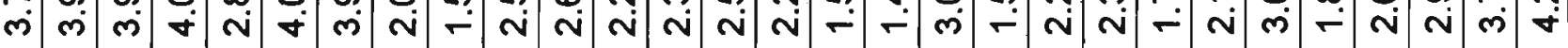

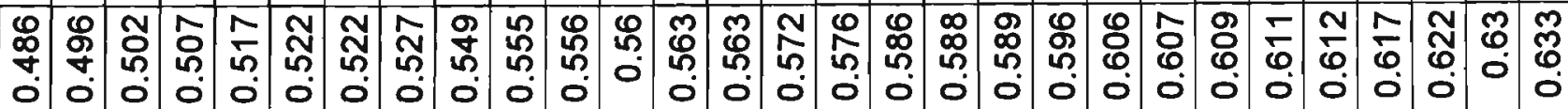

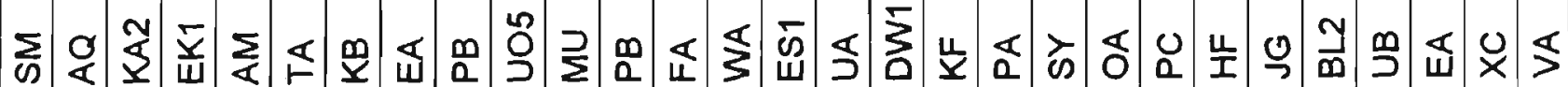

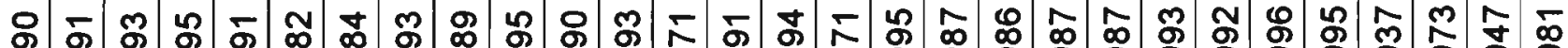

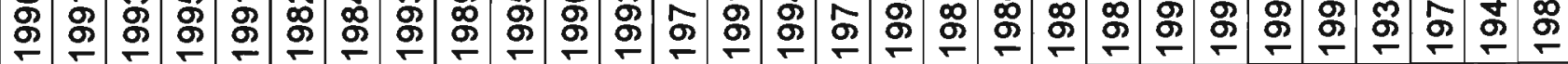

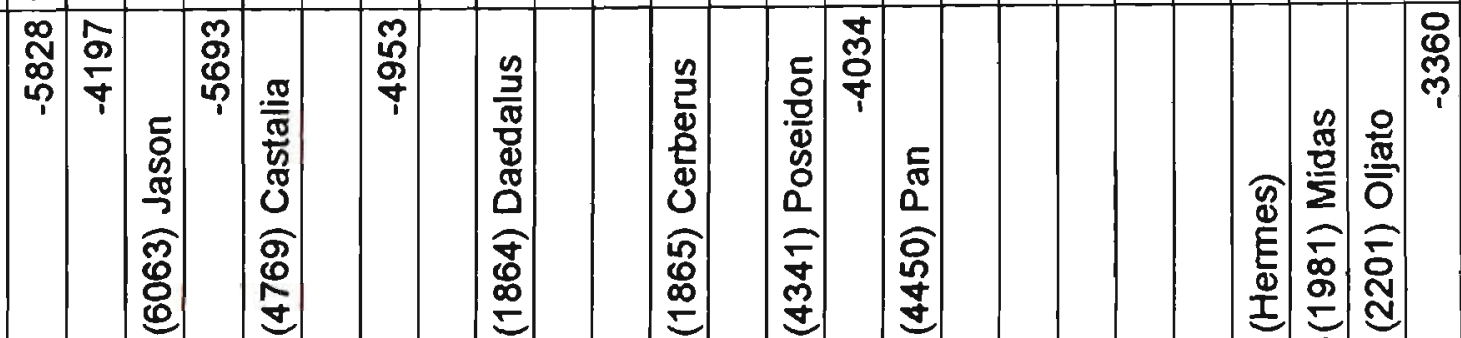




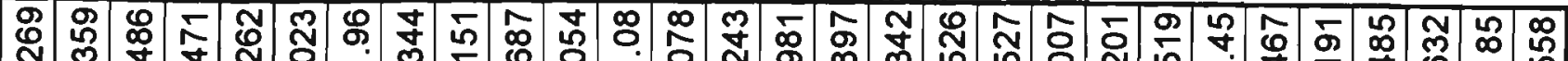

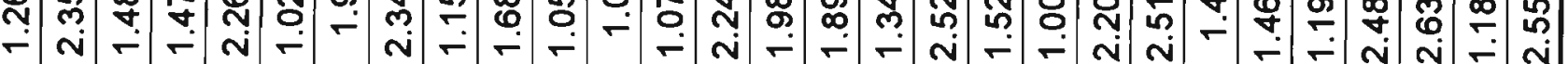
$\frac{0}{\frac{0}{0}}$

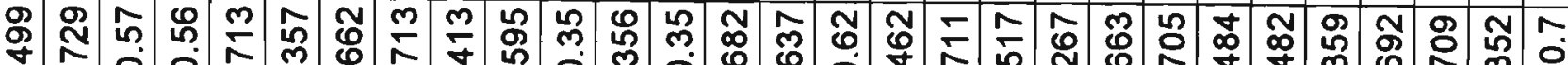

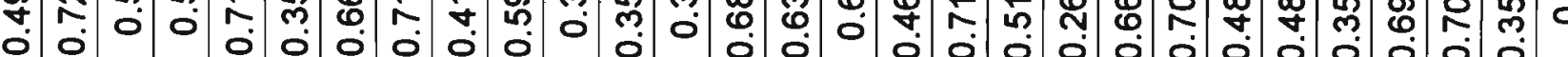
n m

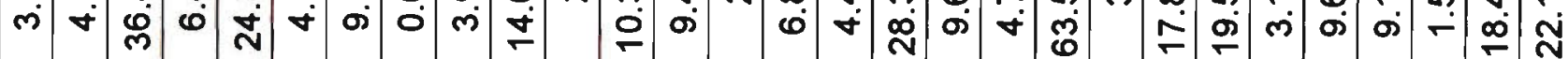

ח

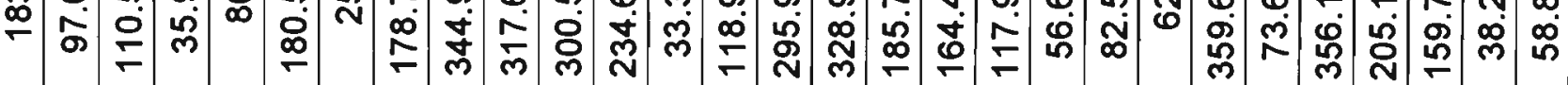

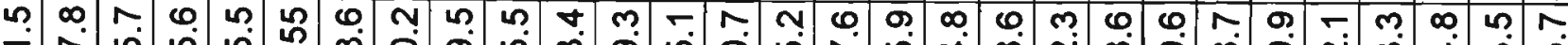
我莳

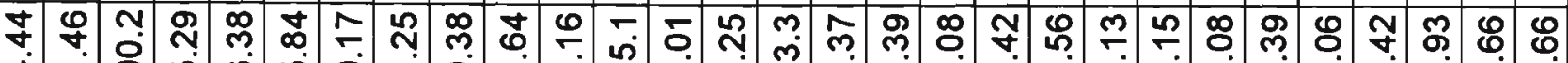
离

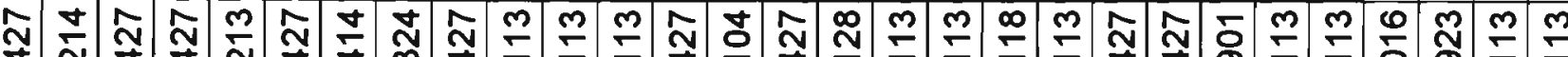

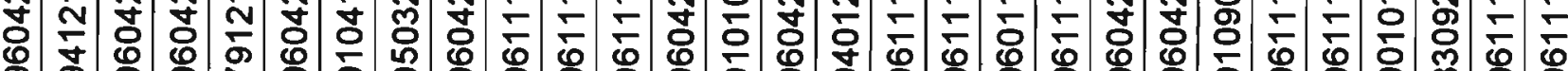

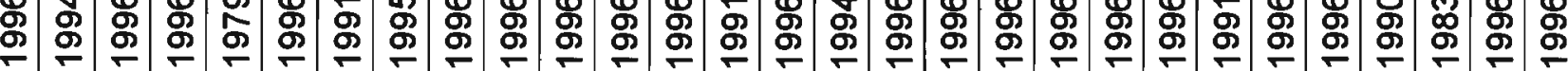

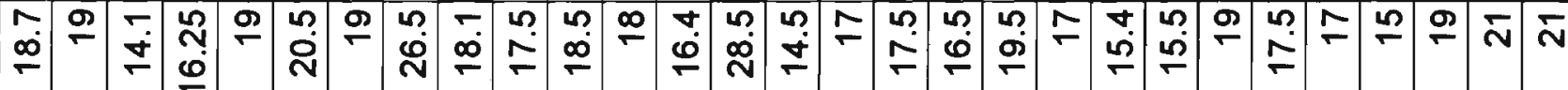

m

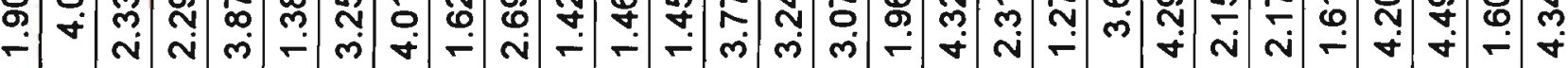

ஜ 6.

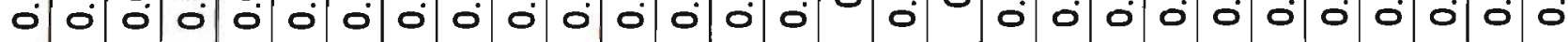

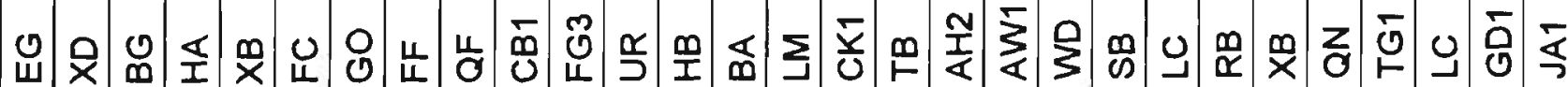
声 怘 :

\begin{tabular}{|c|c|c|c|c|c|}
\hline 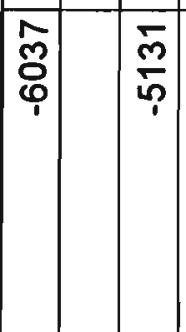 & 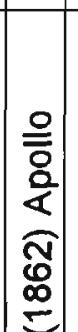 & 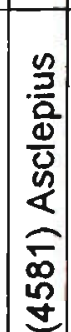 & 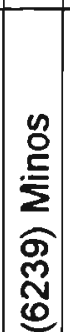 & 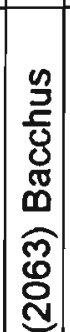 & 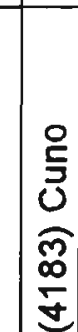 \\
\hline
\end{tabular}

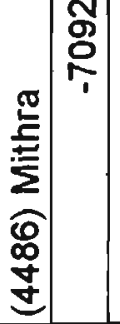


స్N

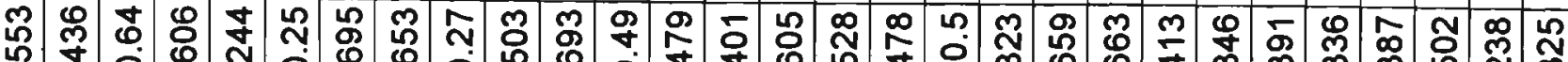

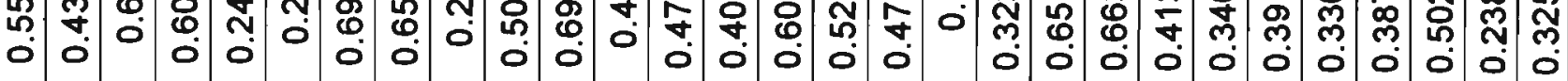

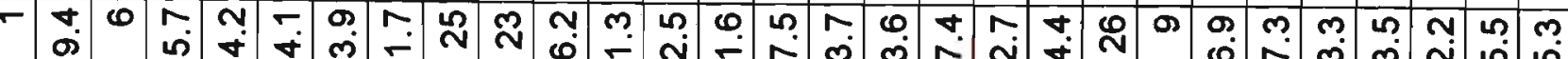

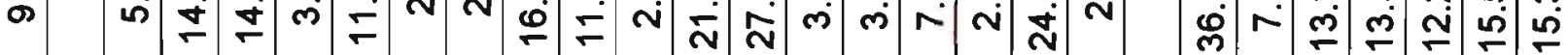

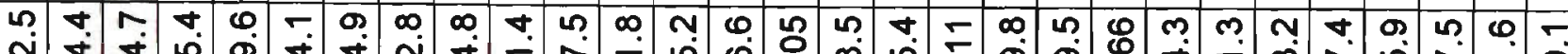

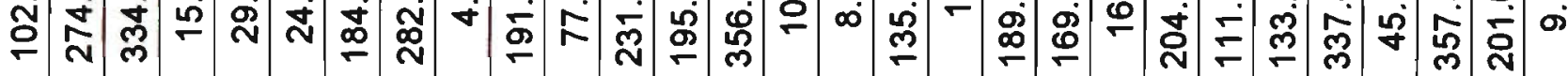
ஸุ

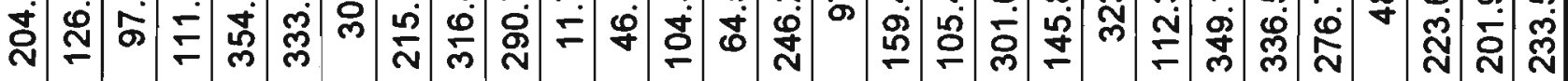

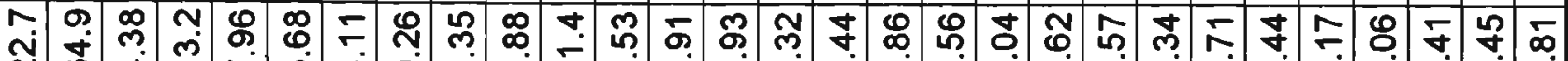

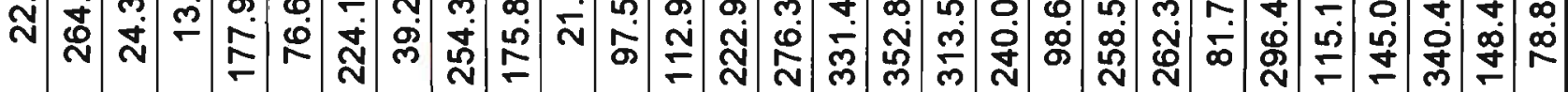

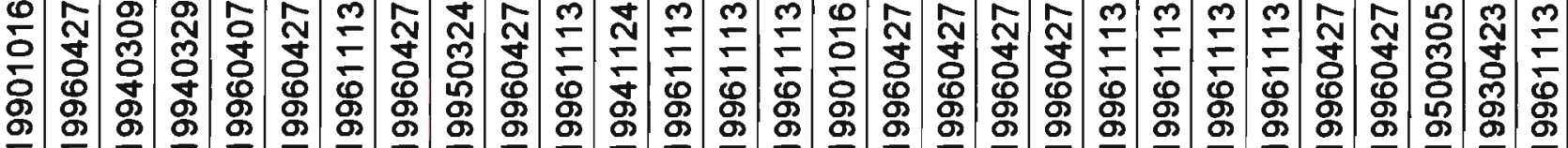
n

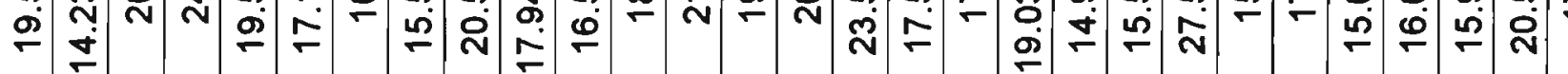

苜

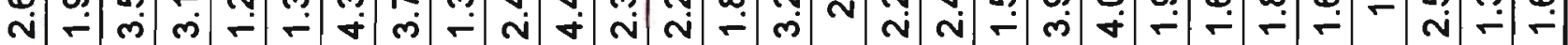

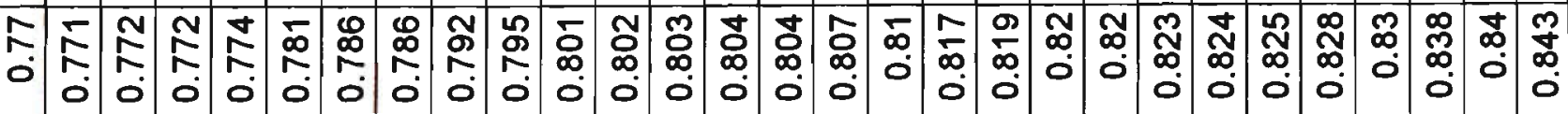

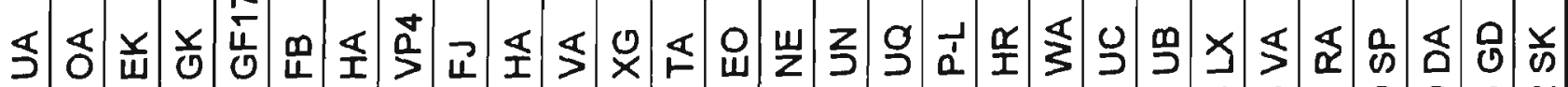

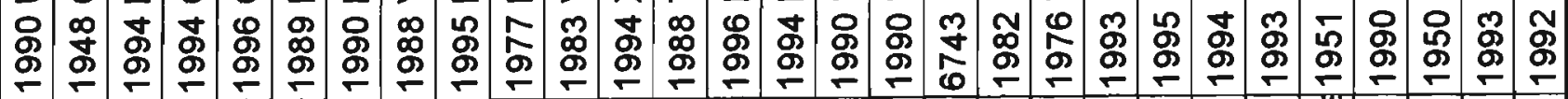

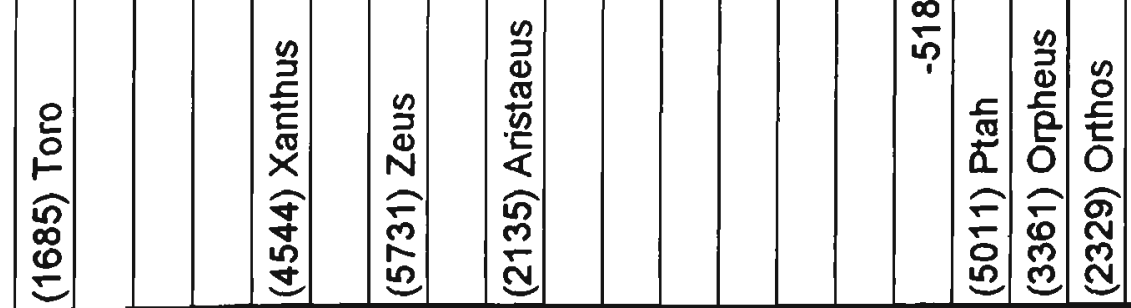

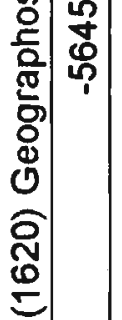




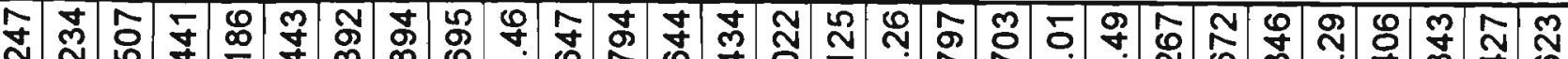
৯

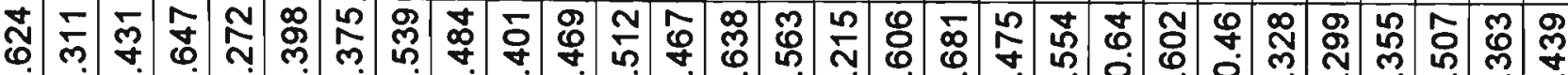

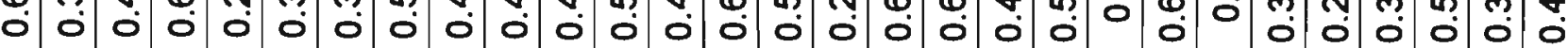

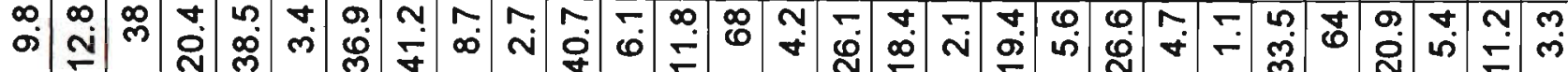
$\sigma \infty m$ m

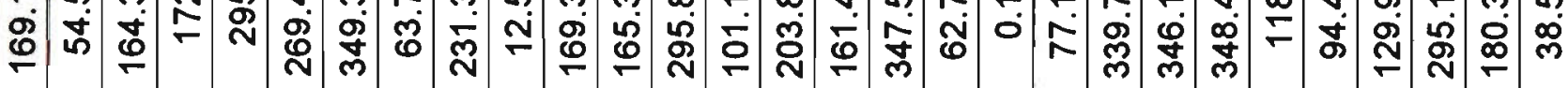

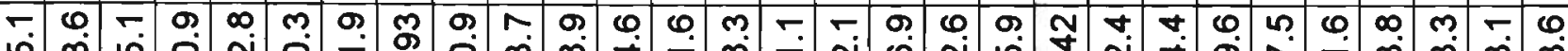

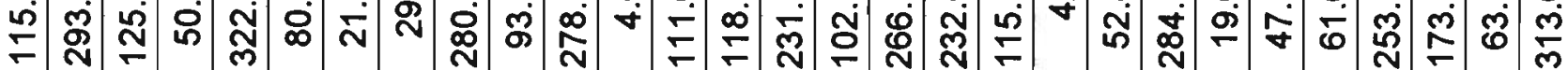

నิ 过

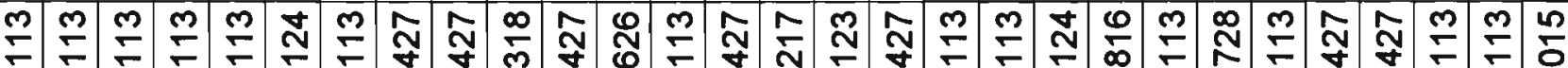

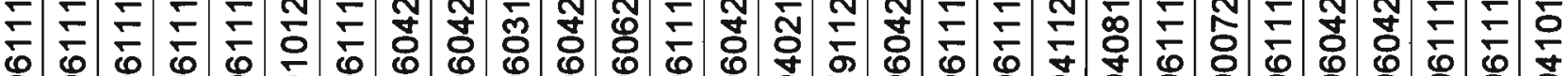
\&

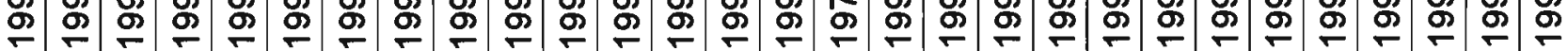

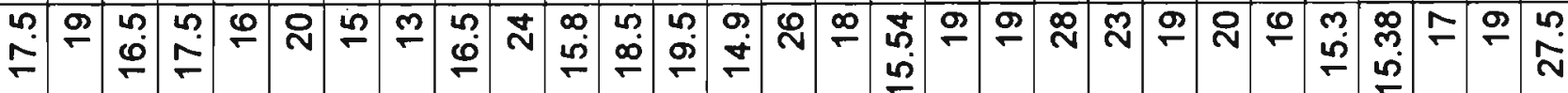

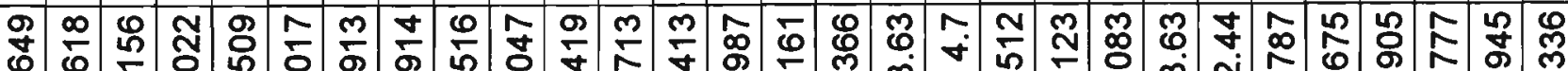

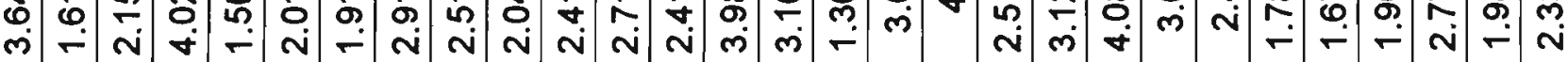

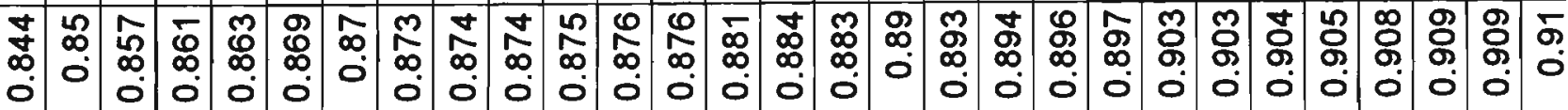

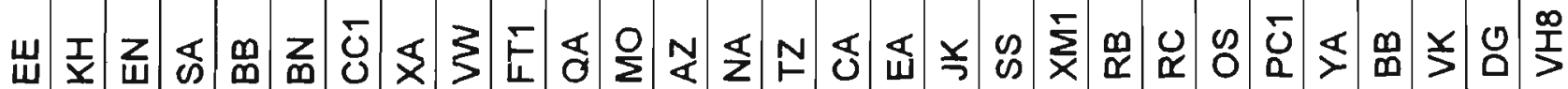
б

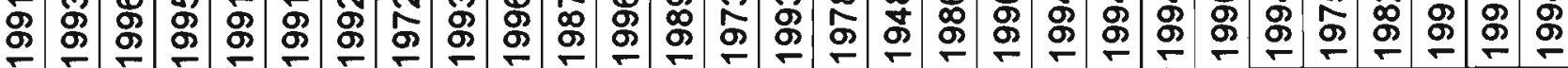

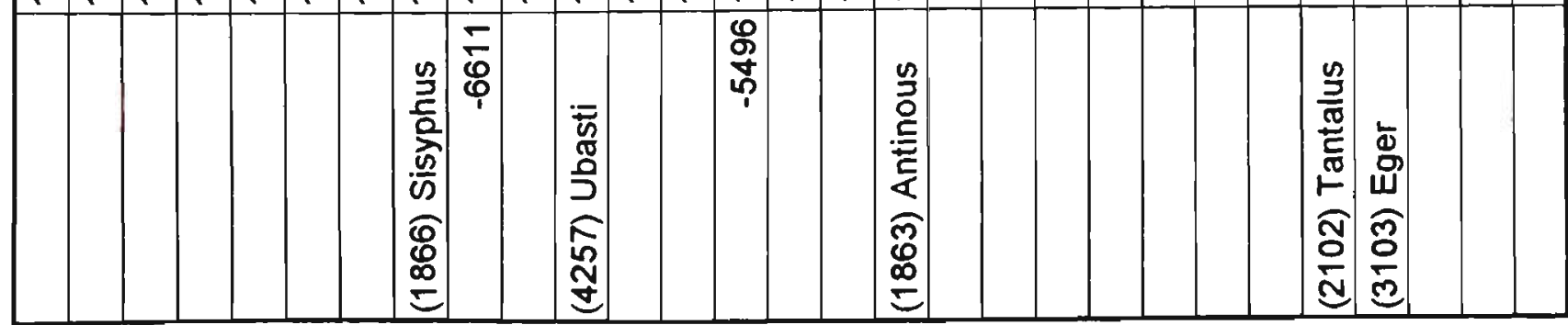




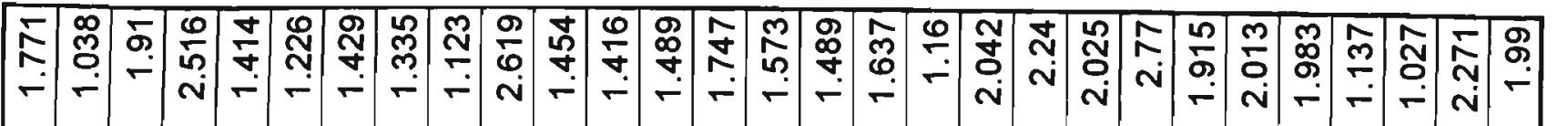

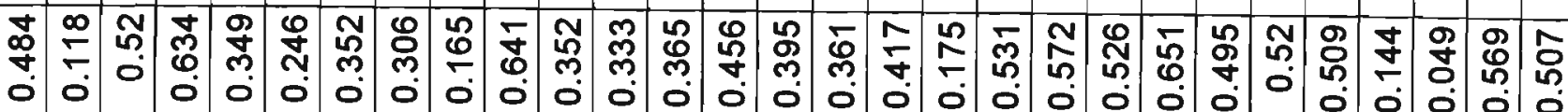

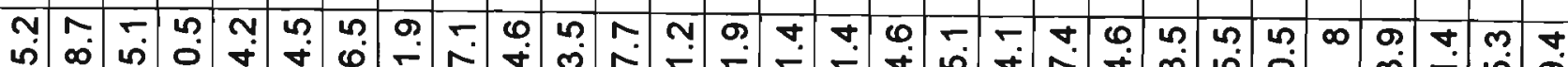

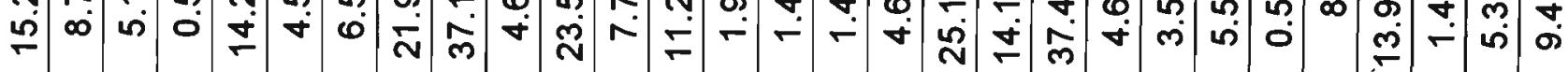

0 - $\sigma$.

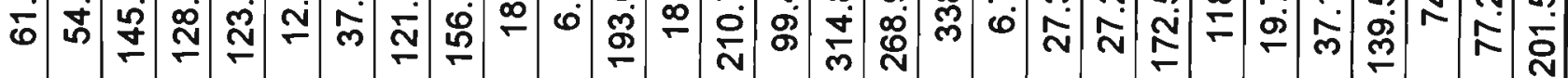

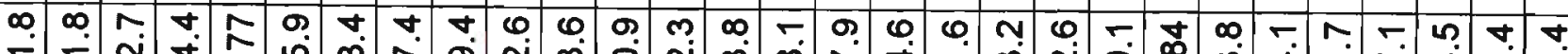

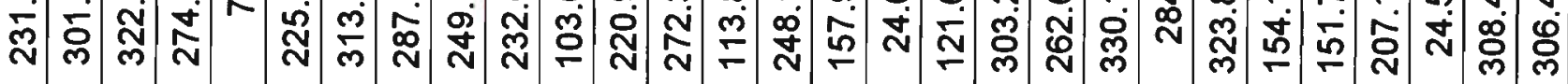

б.

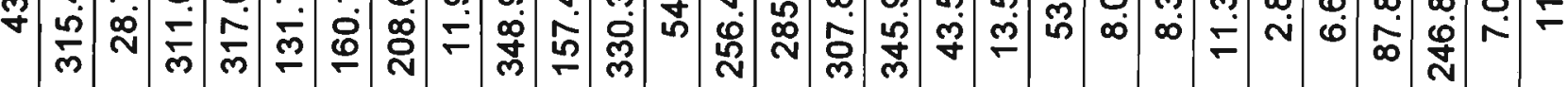

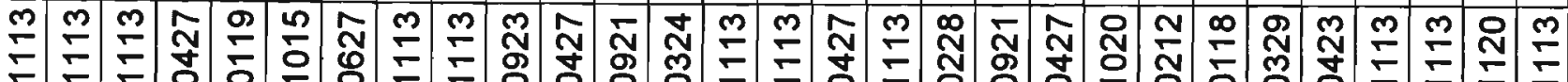

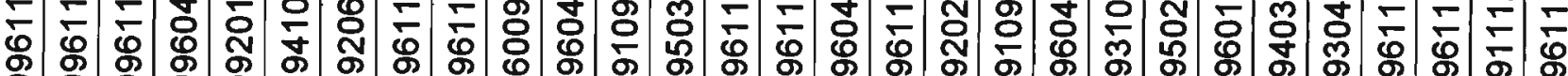
号

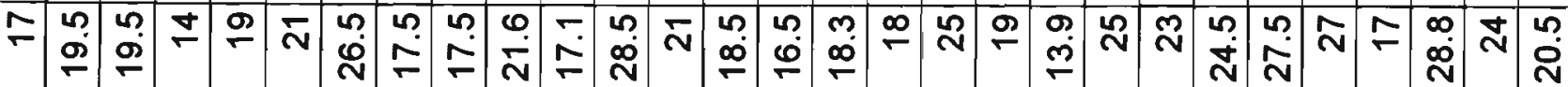

ஸ్రిం

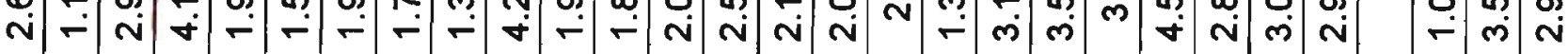

mำำ-

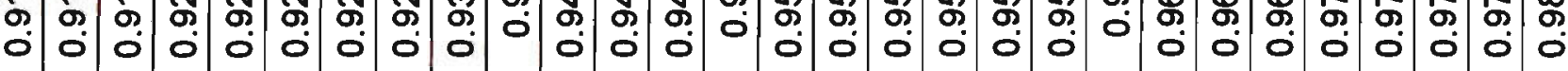

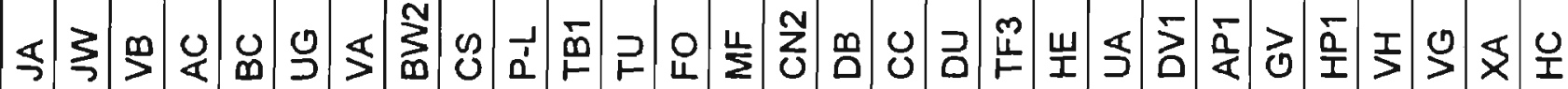
வ ᄃิ

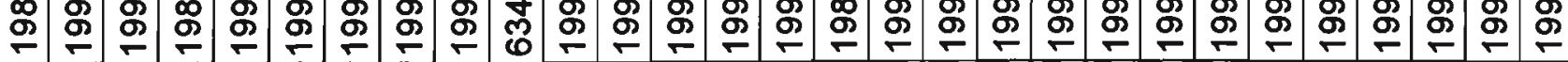

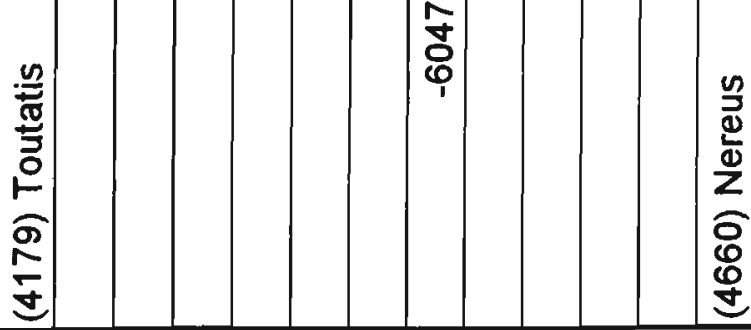




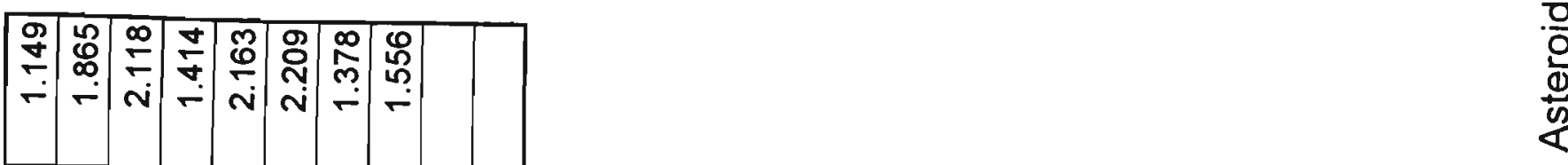

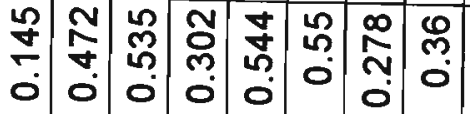

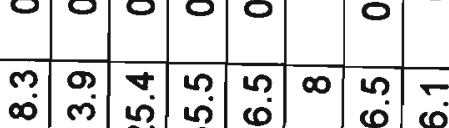

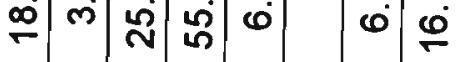

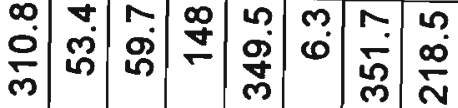

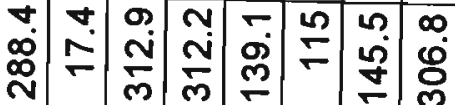

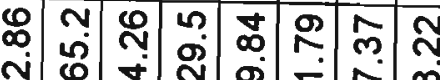

N

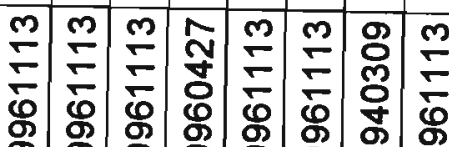

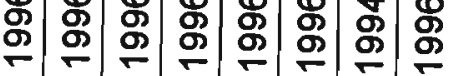

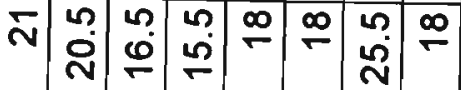

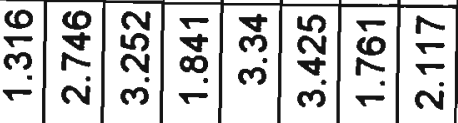

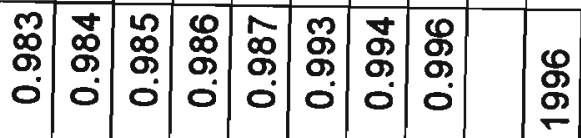

$\stackrel{m}{\circledR}$

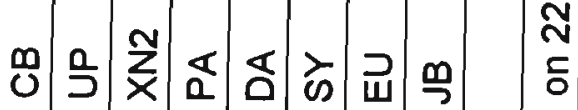

志吕

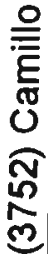




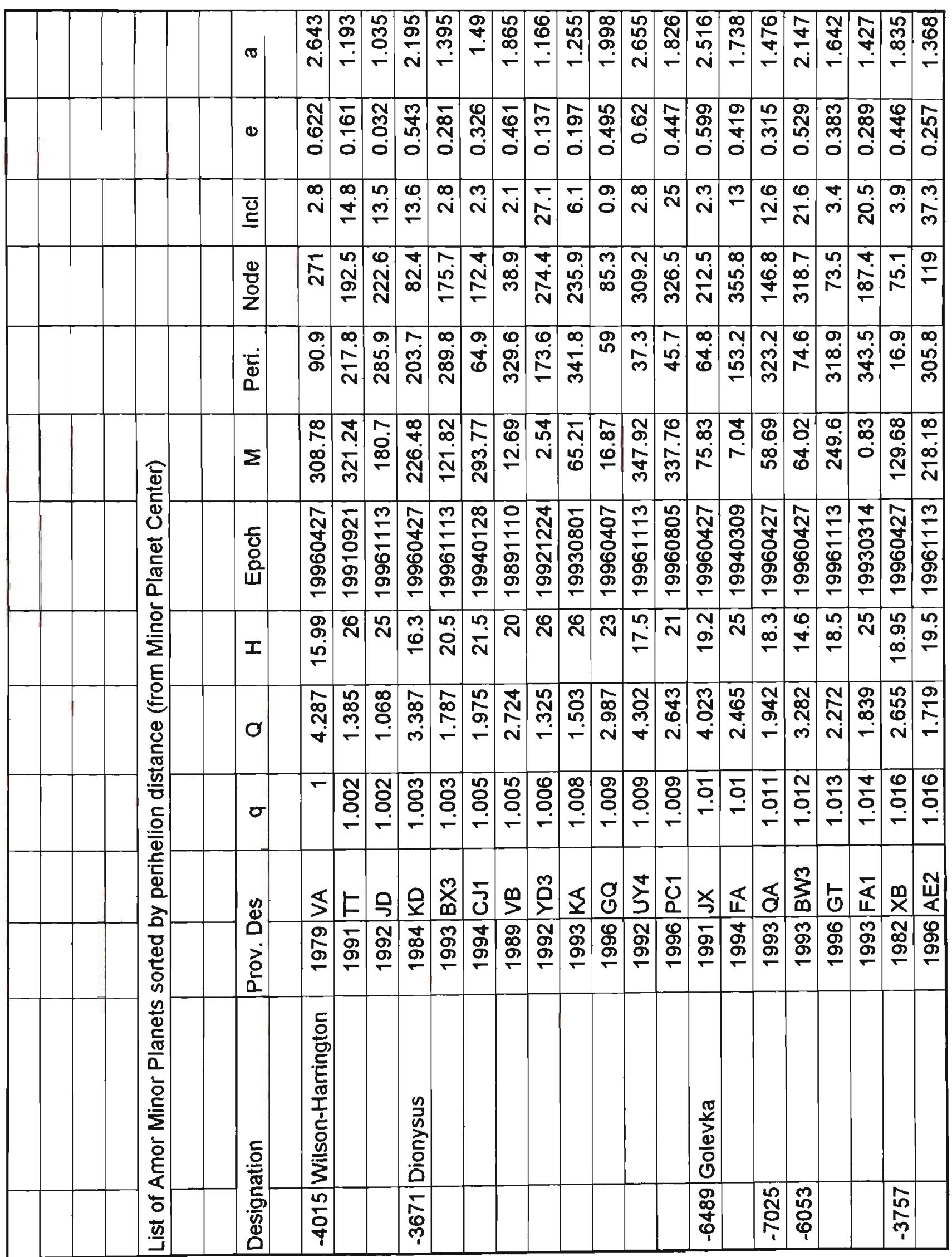




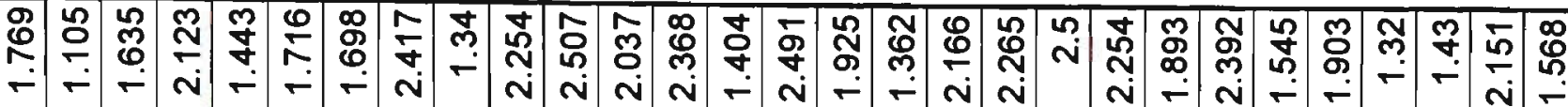
年

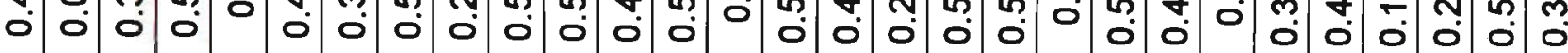
Nุ N ก 每

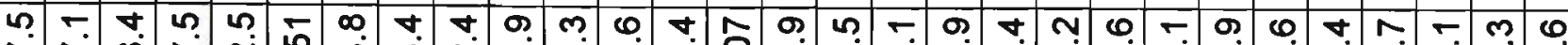
సं $m$ 일 స్లై

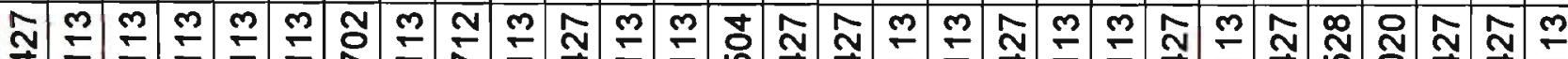

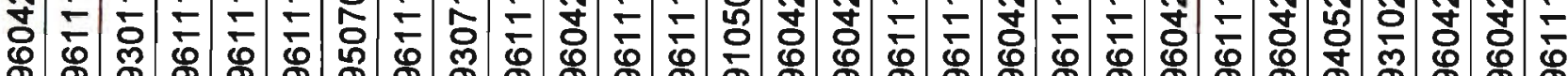

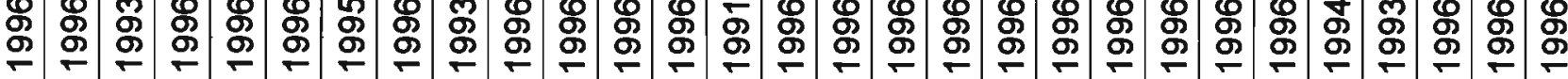
ก

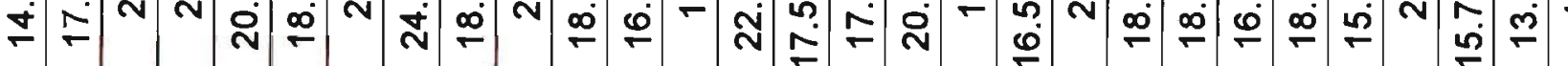

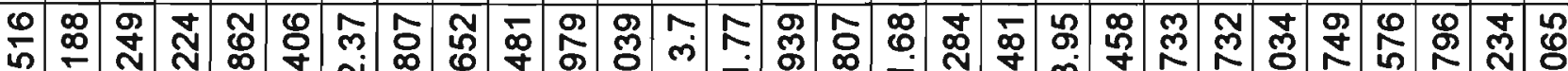

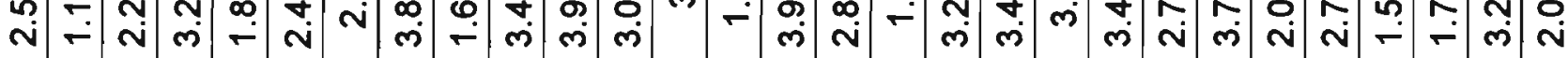

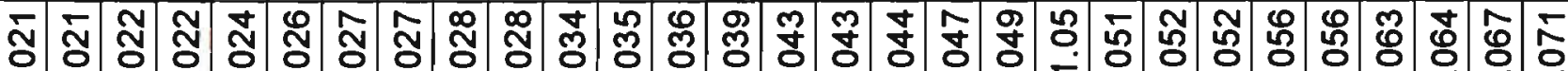

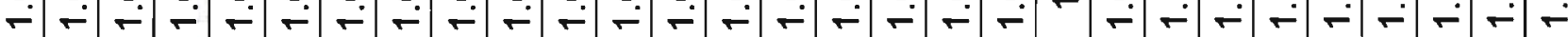

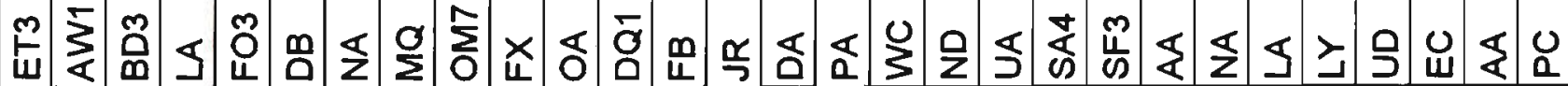

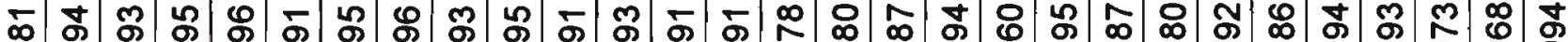
:

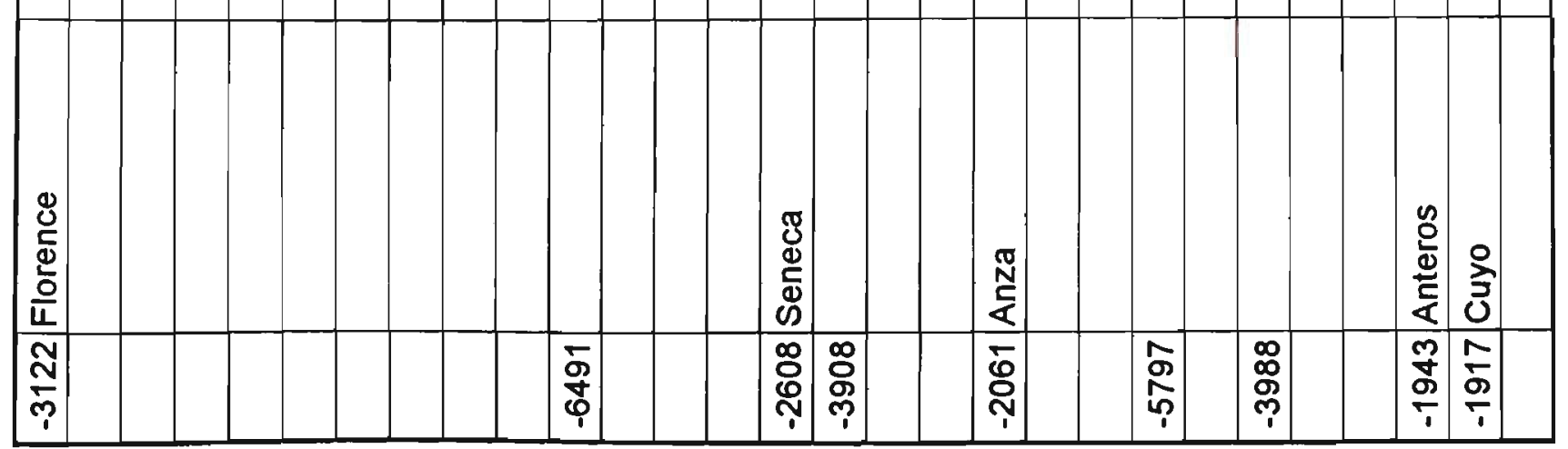




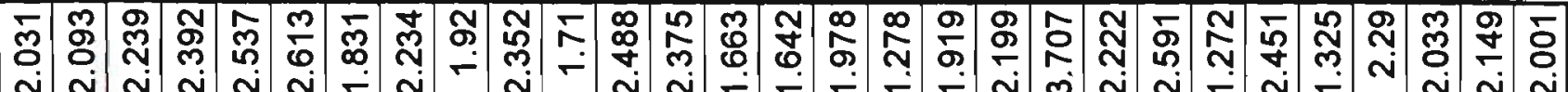

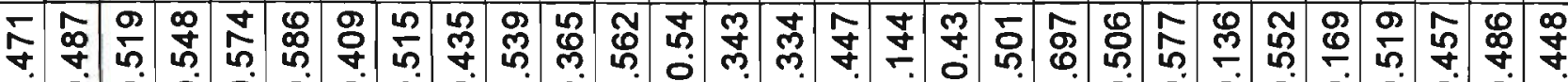

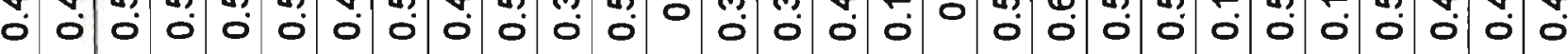
$\neg$ l $-\infty$ 少舟 n 0 n

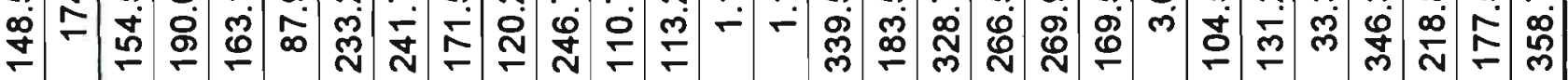

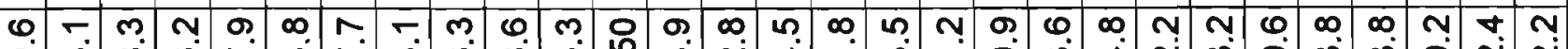

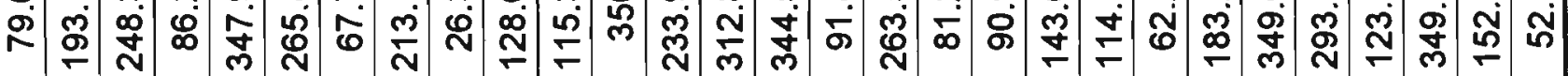

హ゙ ம்

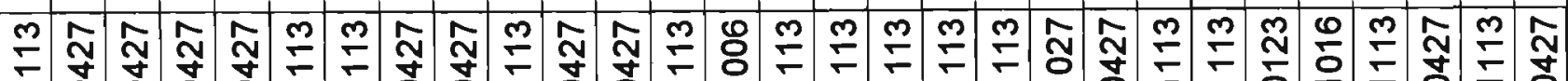

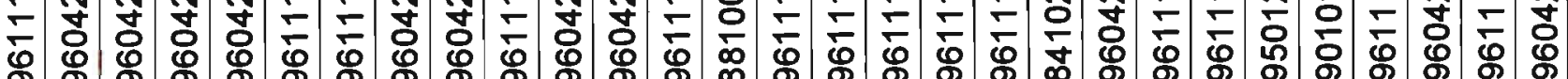
ᄋ ᄋ స̄

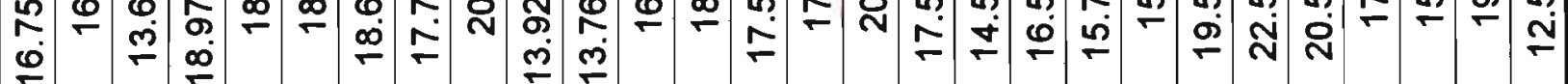

总

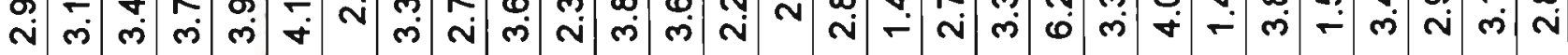

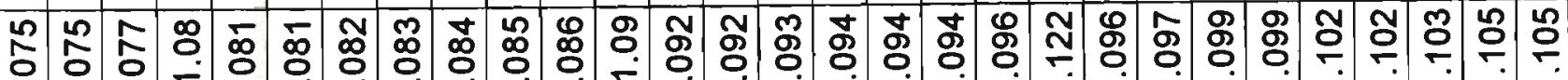

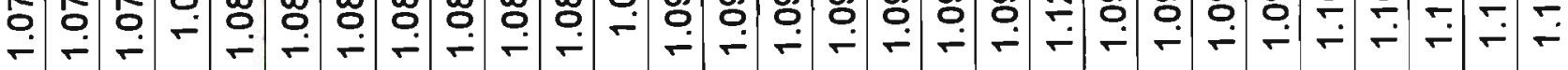

曽全 O ภ

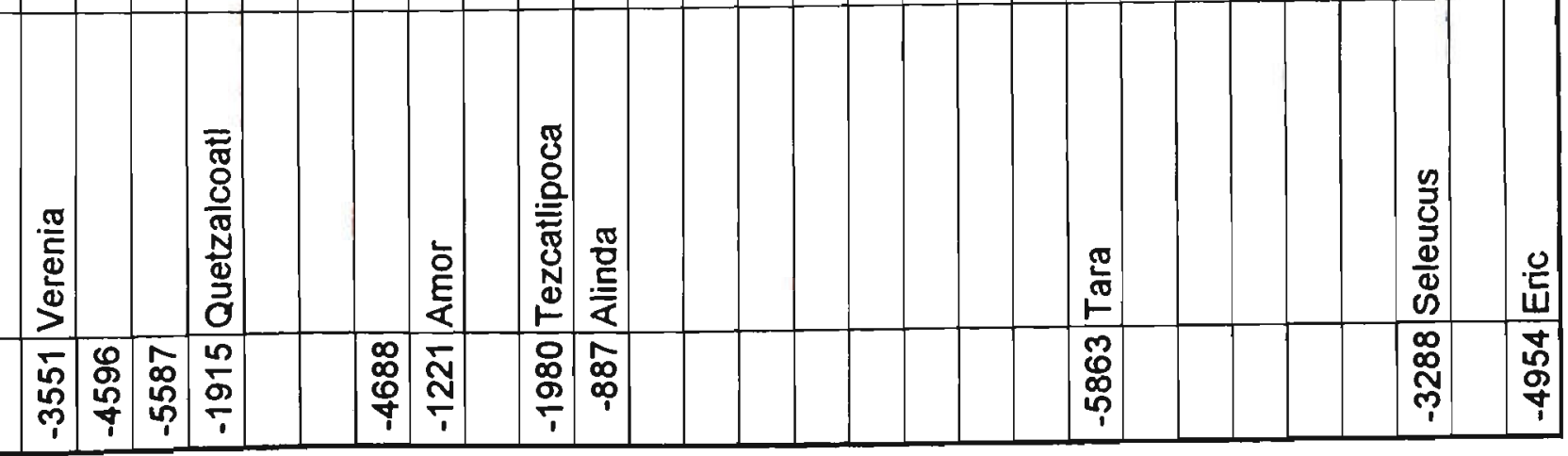


芯

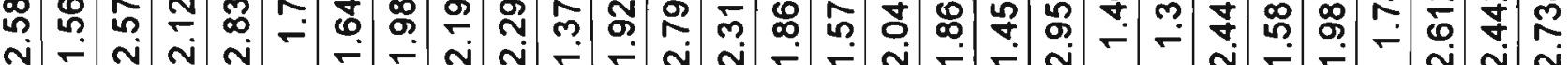

N

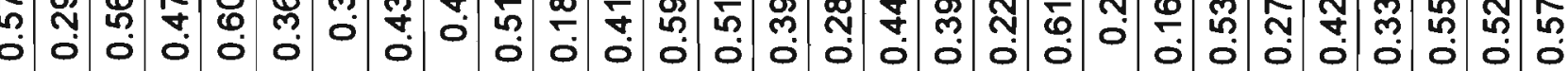

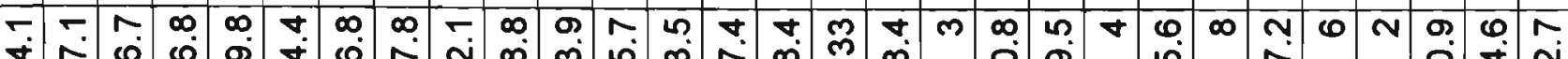

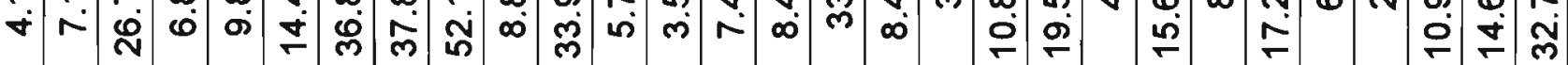

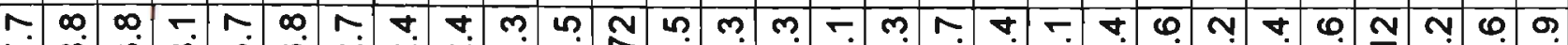
N ก + t

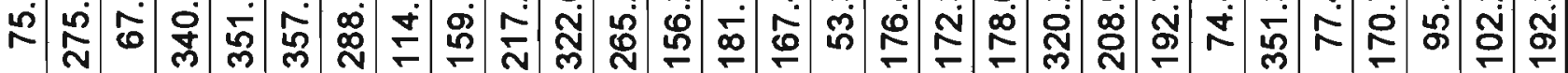

凹 일 $\stackrel{\infty}{f}$ ம

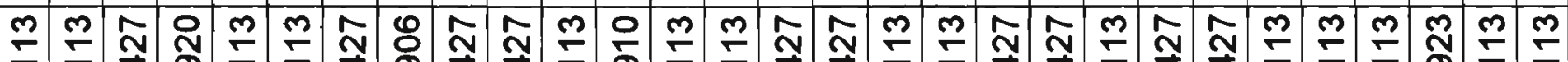
审 它客

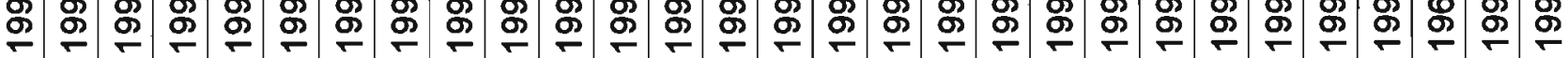

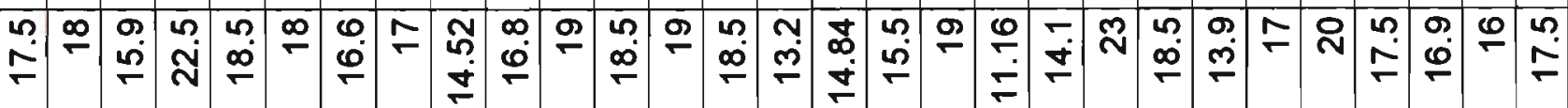

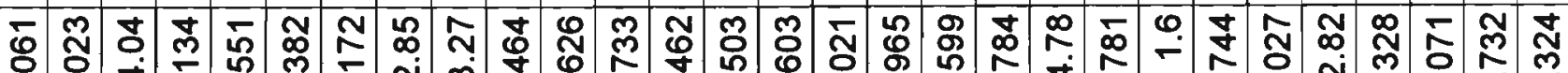

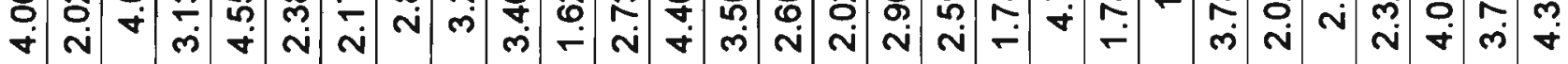

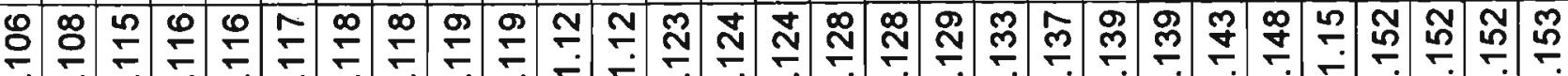

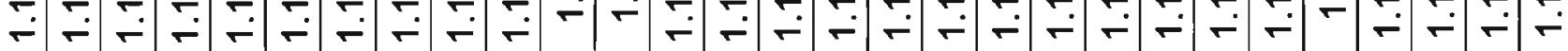

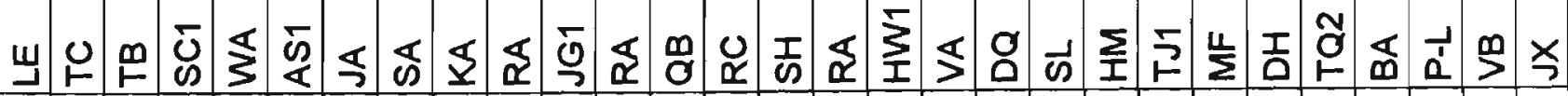
L ᄋ 


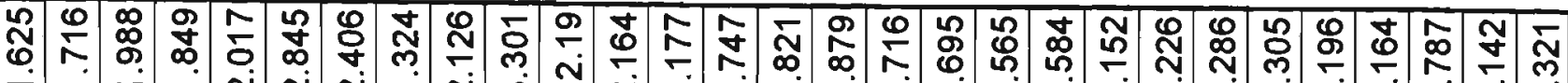

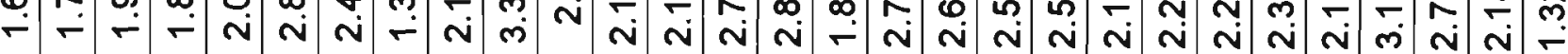
ஜ

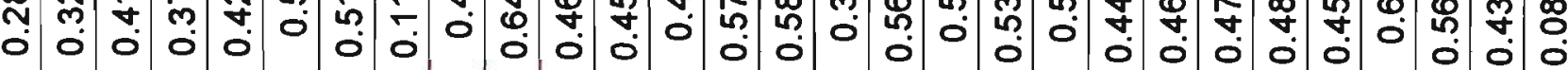

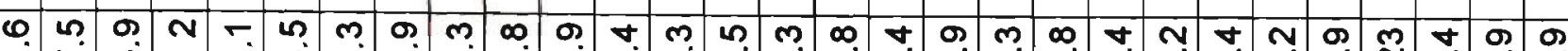

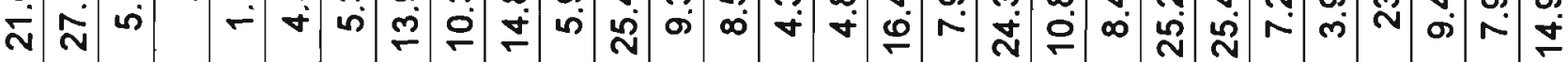
O

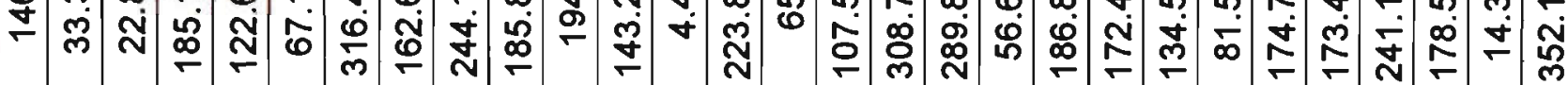

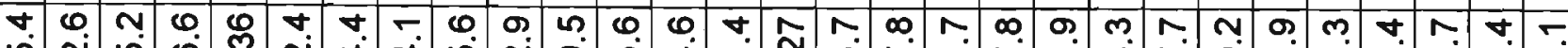

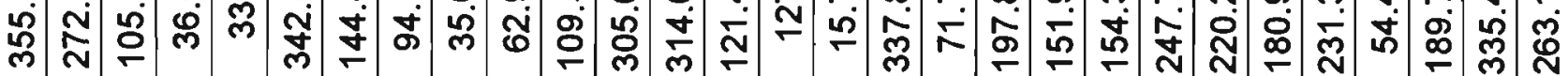

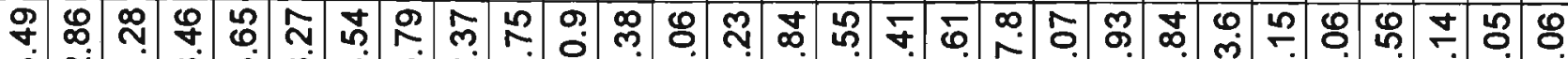

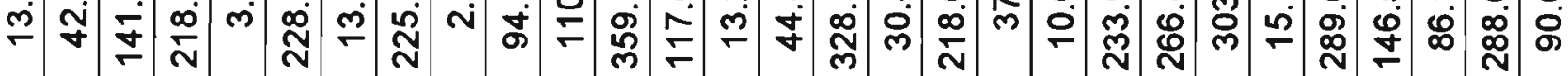
N

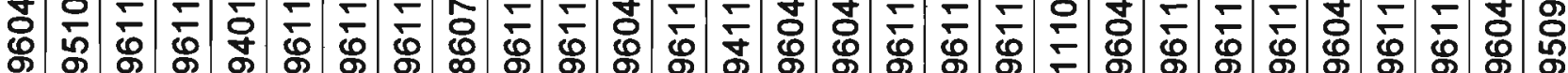

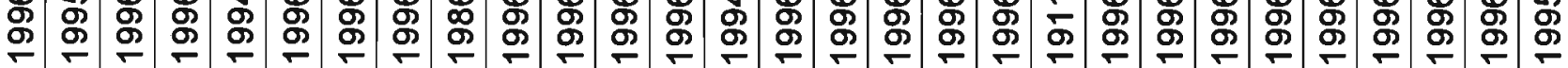

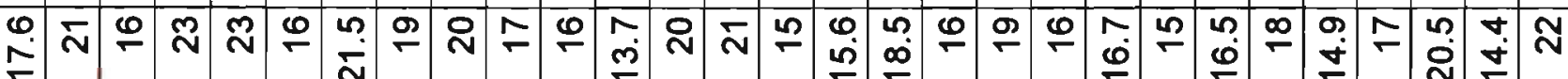

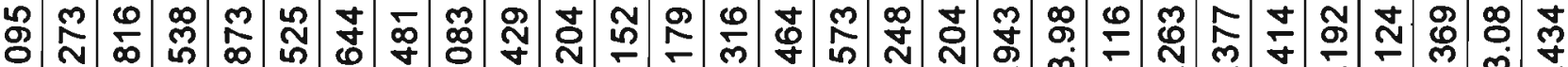

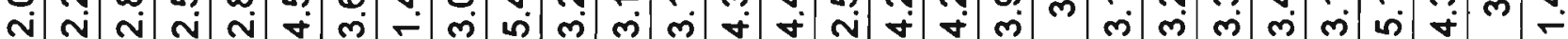

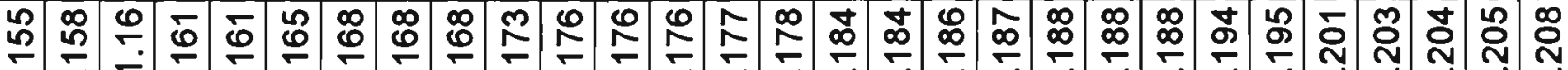

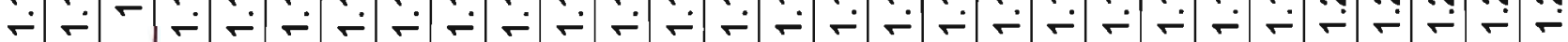

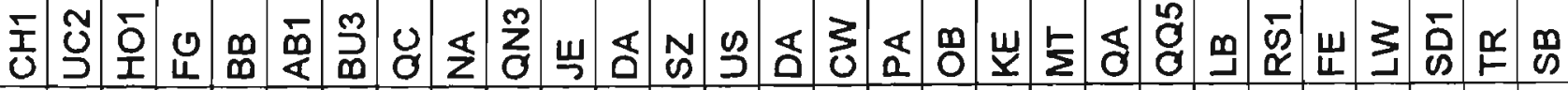
N

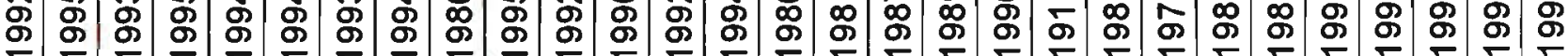

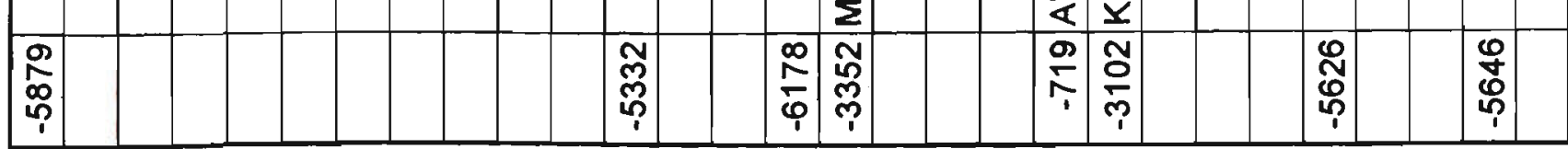




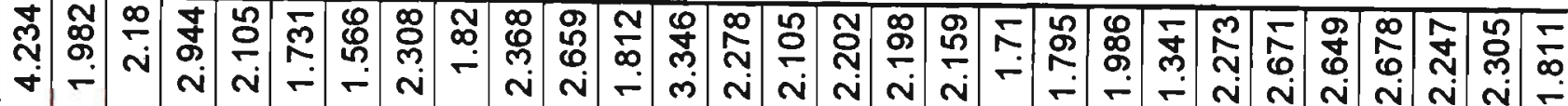

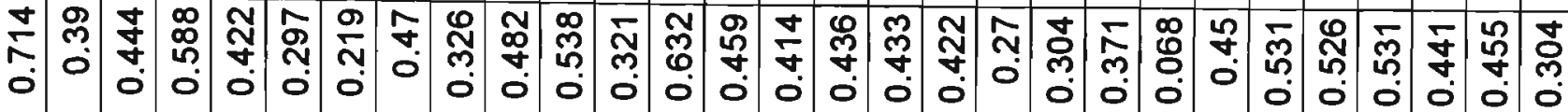

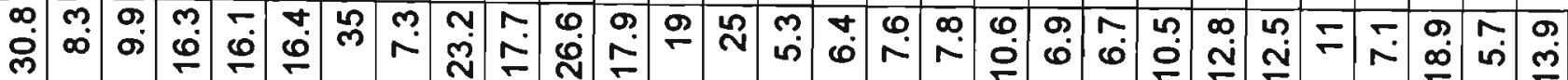

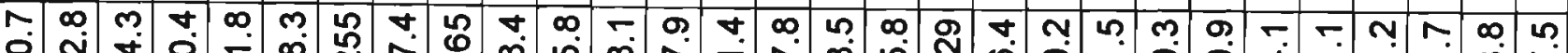

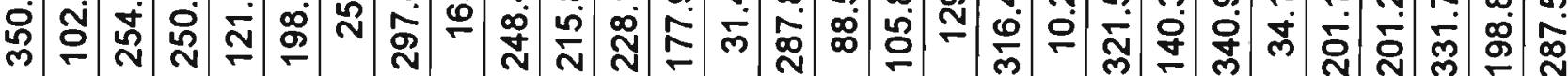

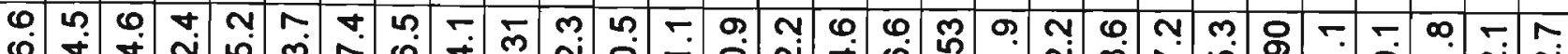

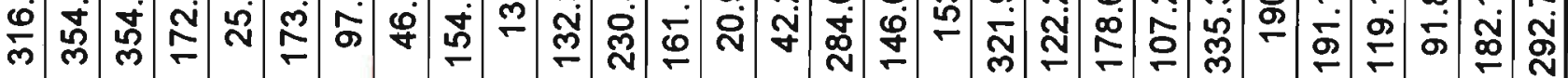

啰

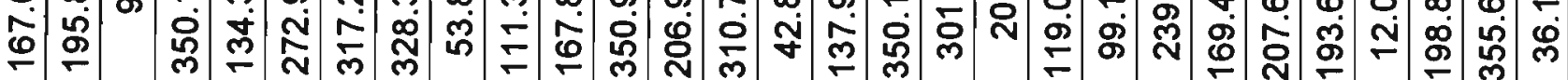

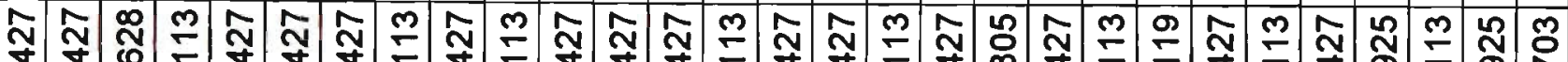
宑 审

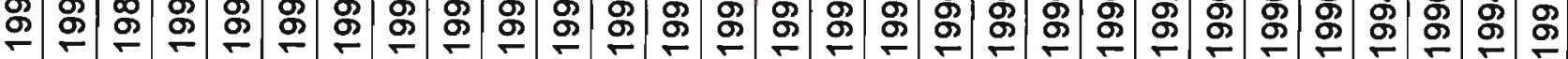

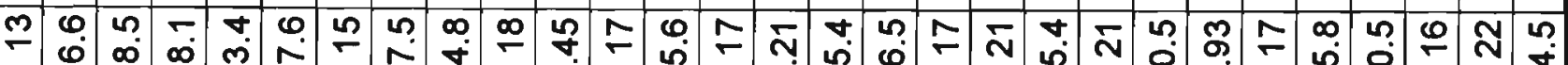

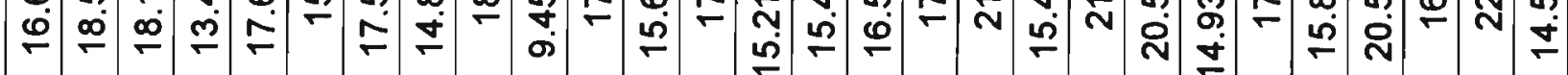

ஸ̂̉

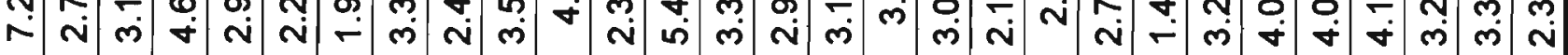

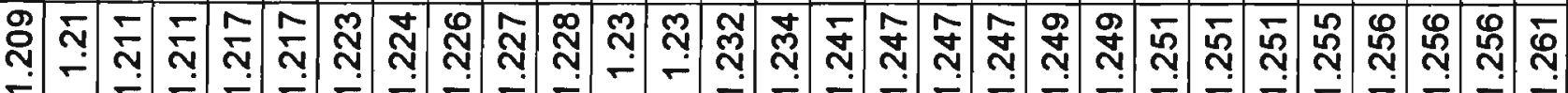

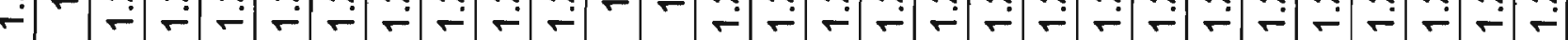

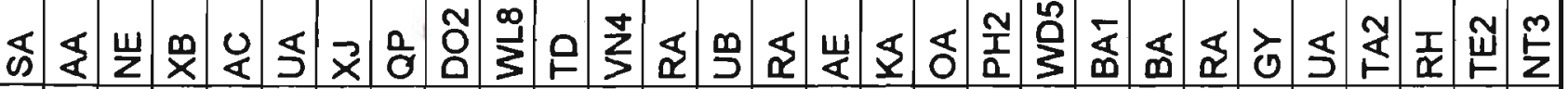

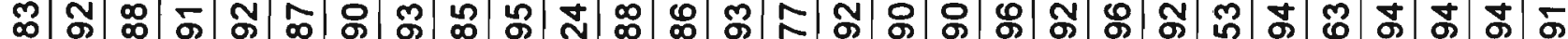

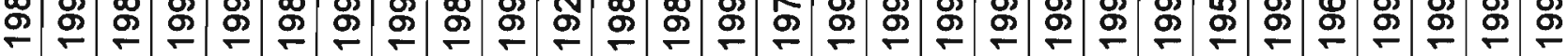

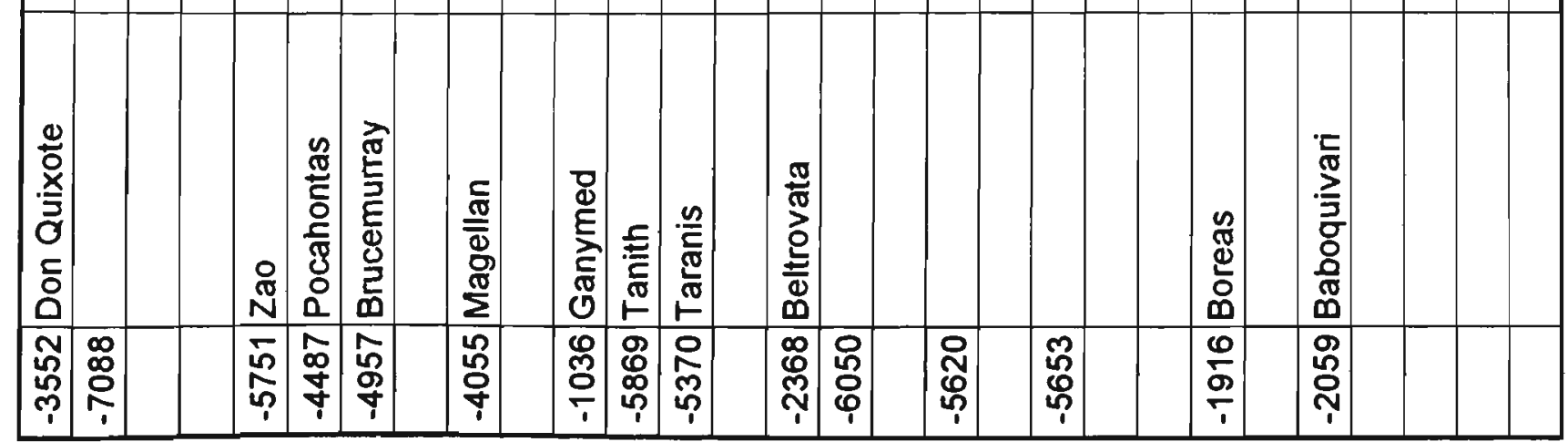




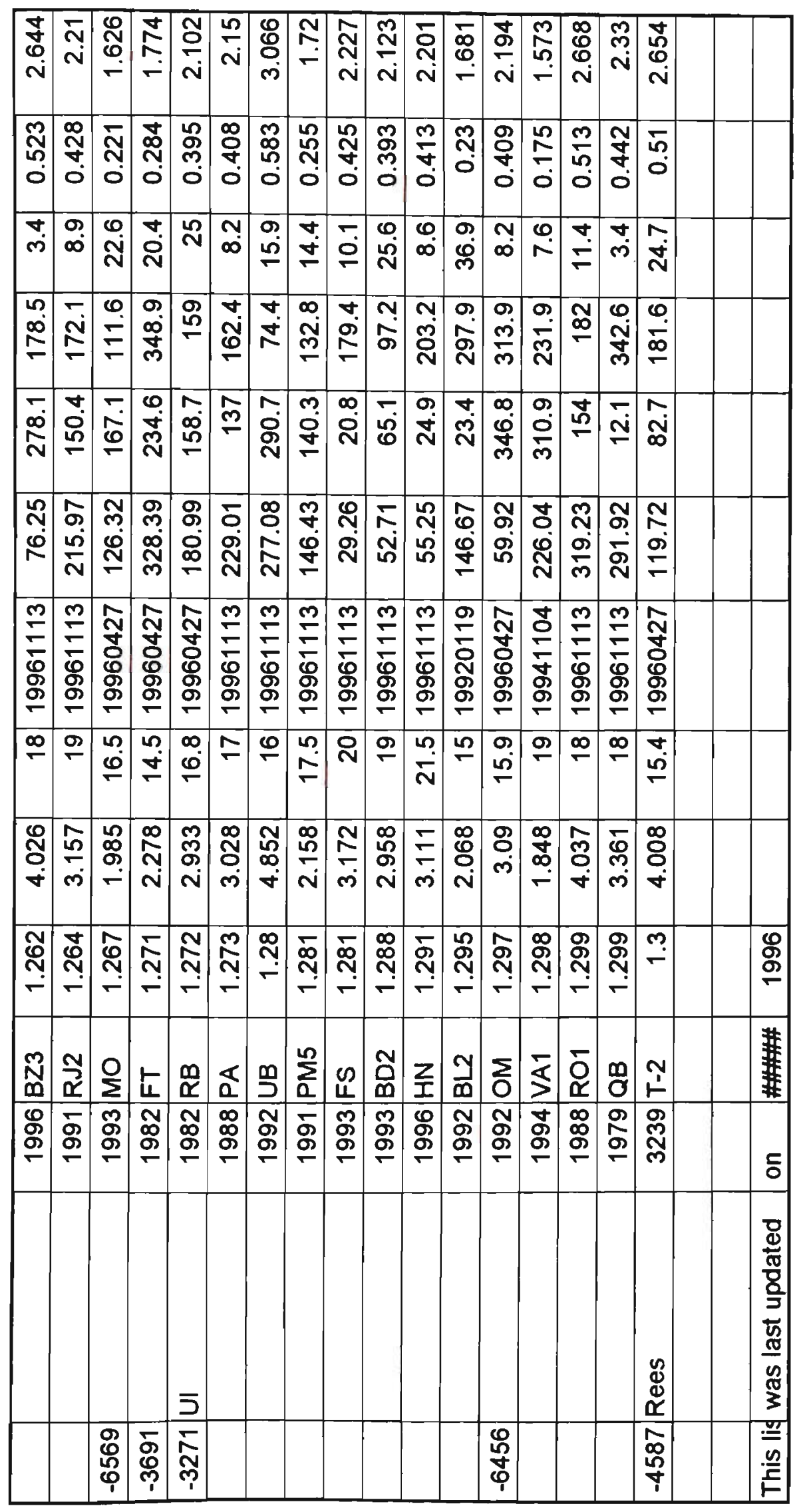




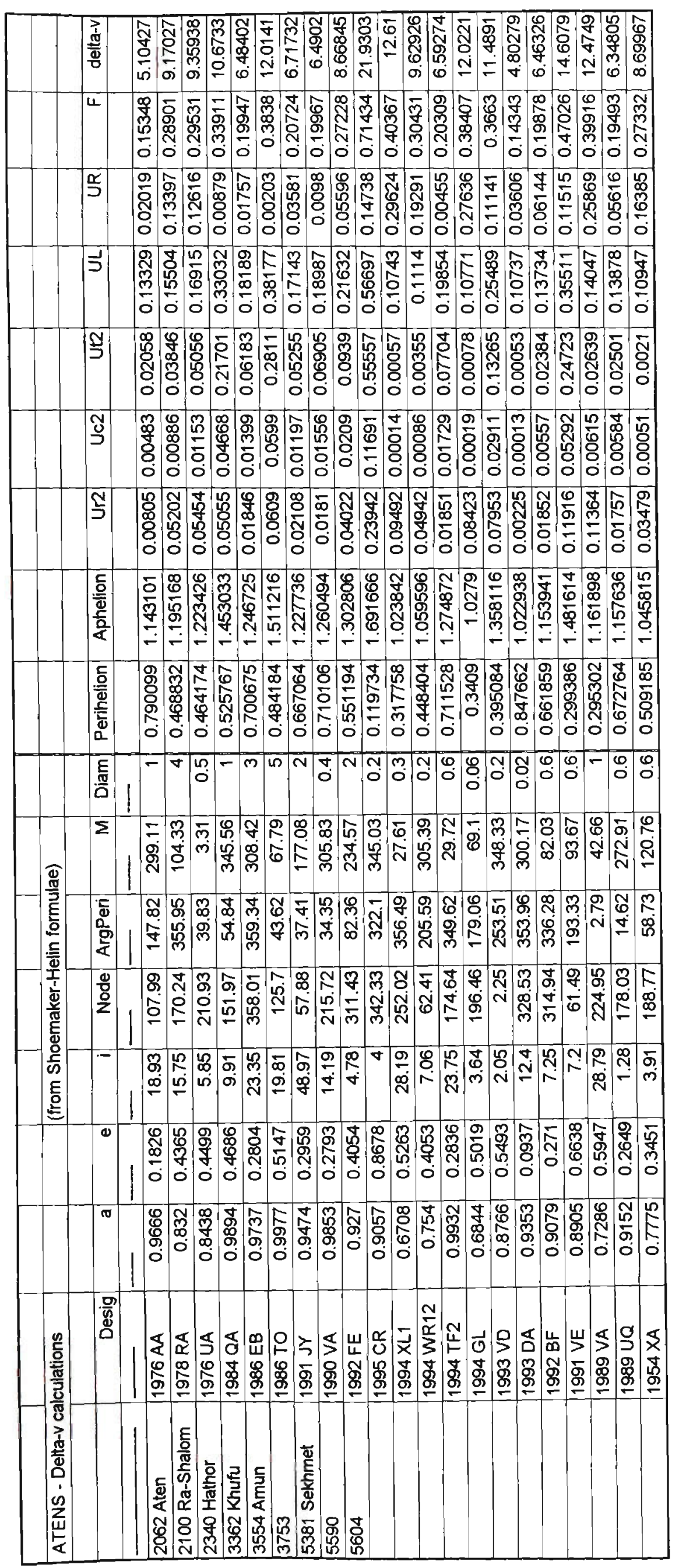




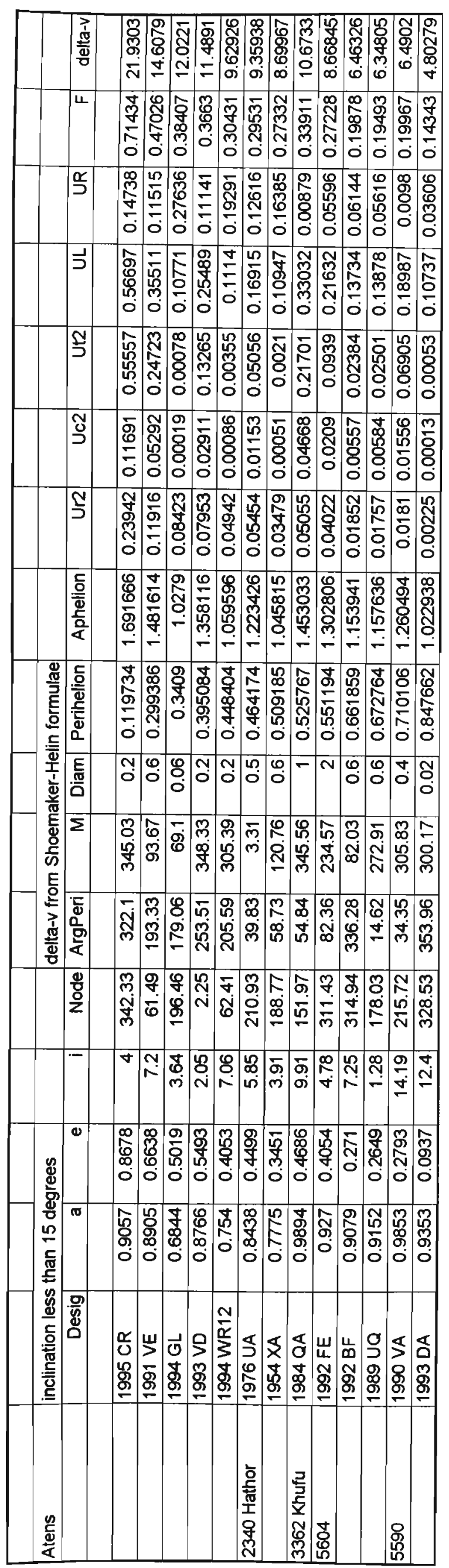

$\stackrel{N}{N}$ 


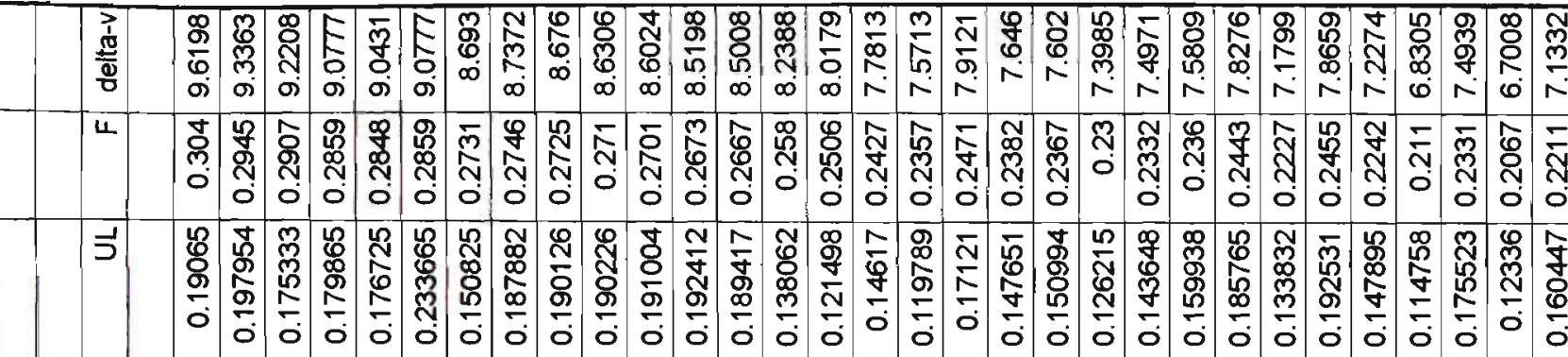

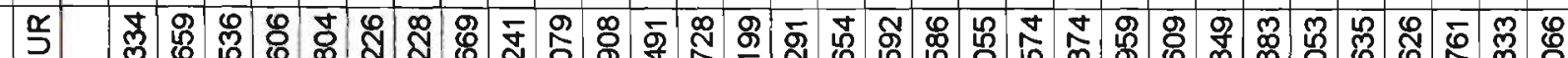

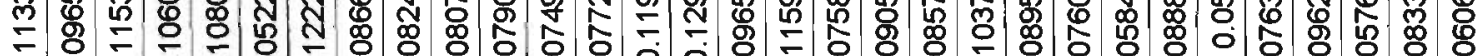
○.

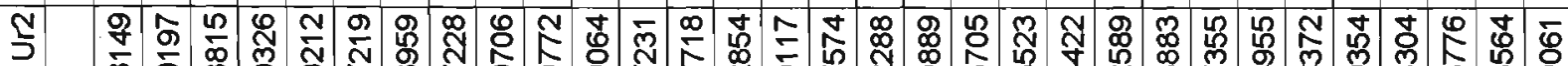

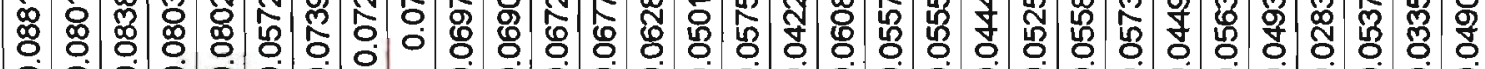

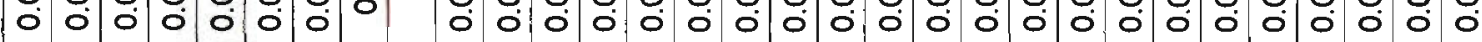

งู

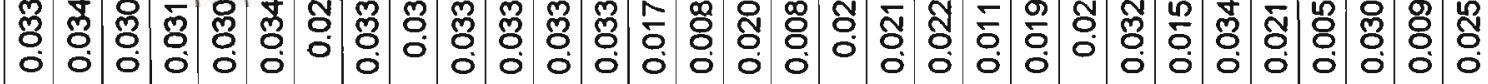

ᄀ) \% 0.

产 है

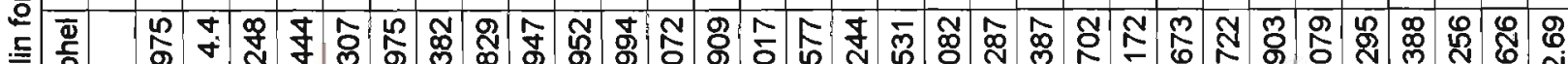

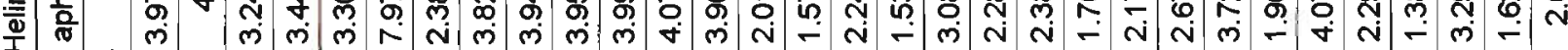

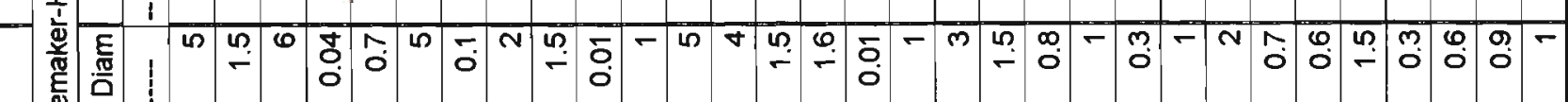

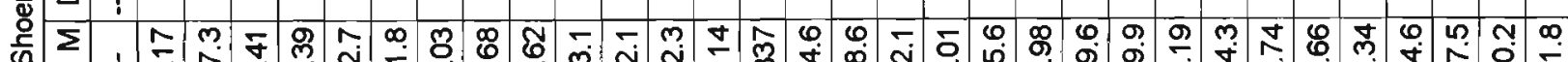

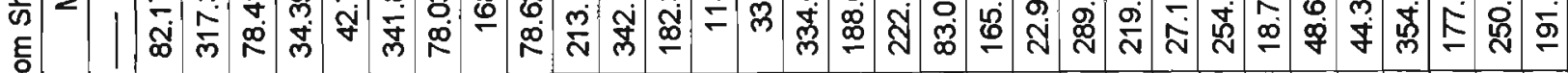
紊

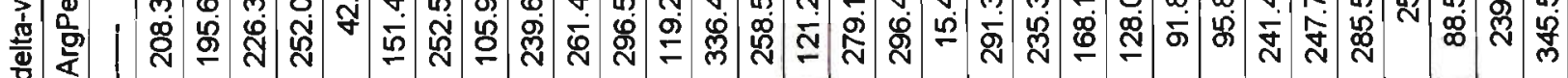
\&

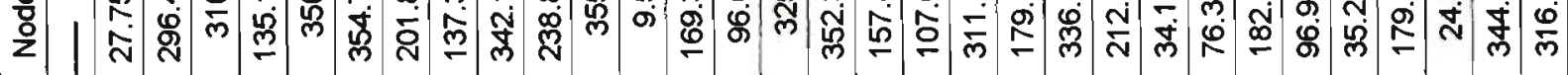
œ

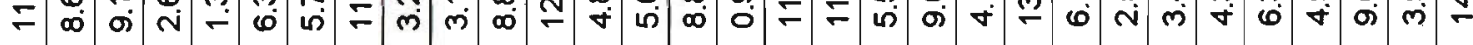

-

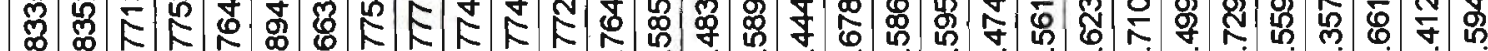

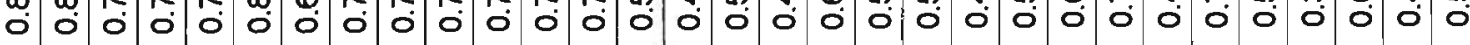

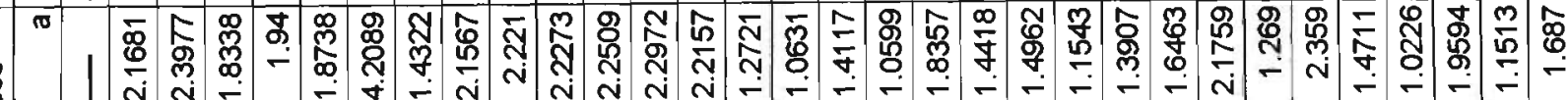
客

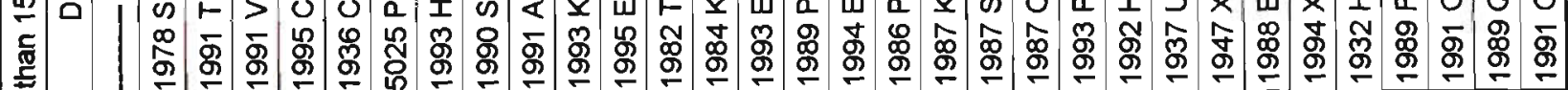

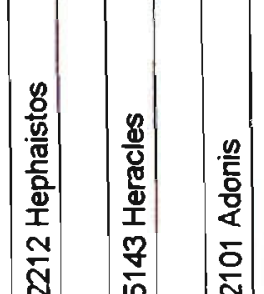




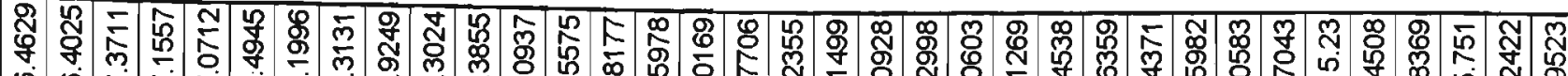

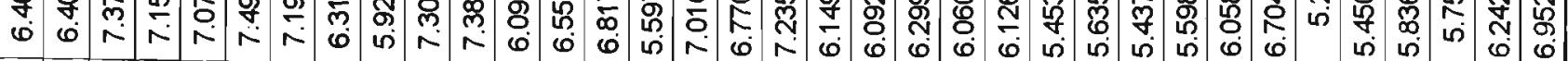
兽

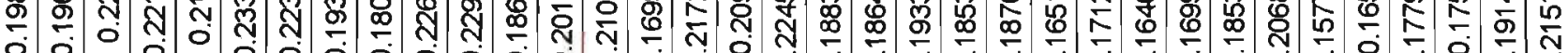

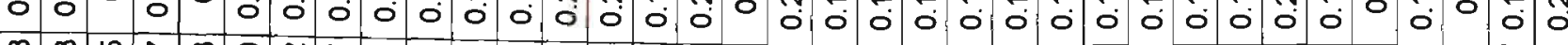
守 壬至

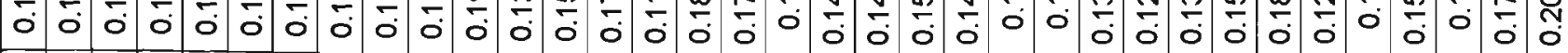

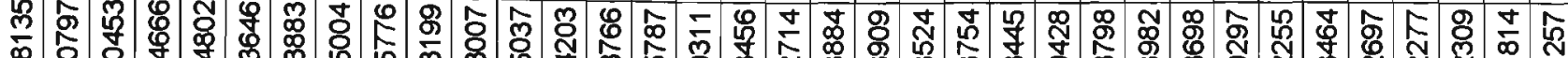

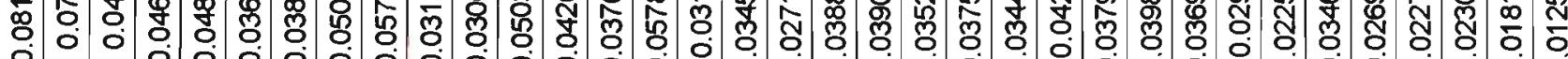
$\circ \circ \circ \% \circ \%$

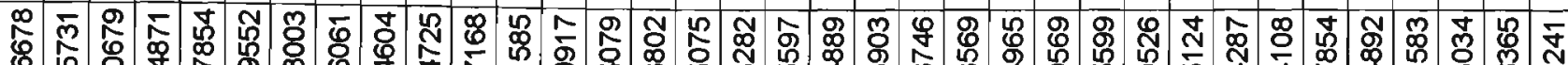

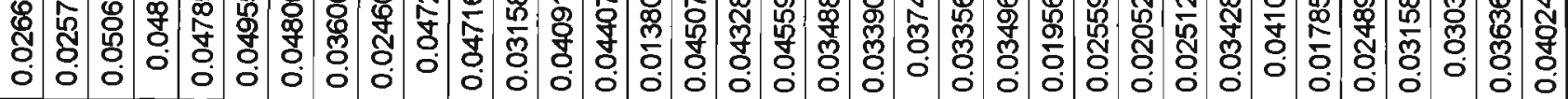

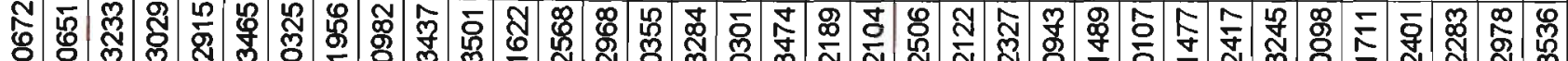

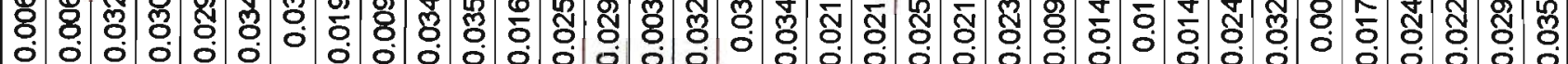

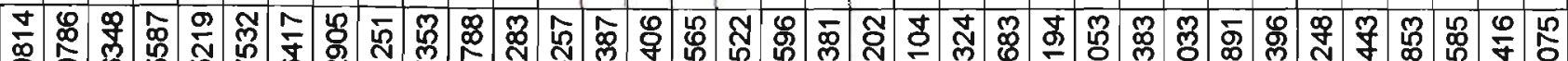
8. 영 $\circ \%$

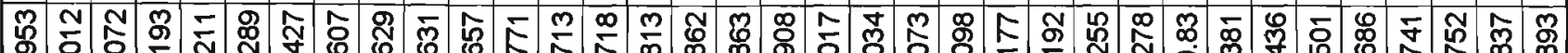

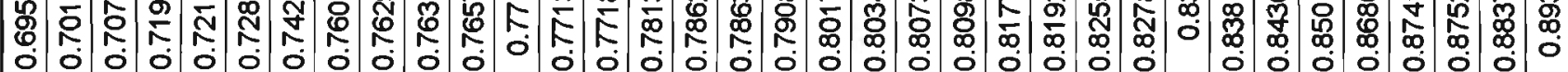

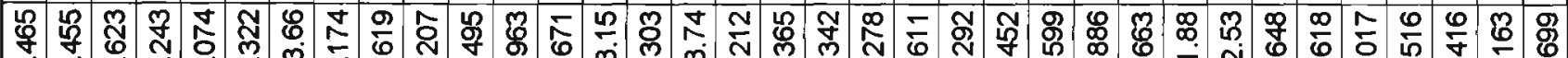

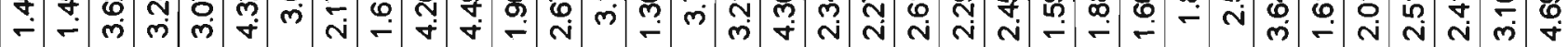

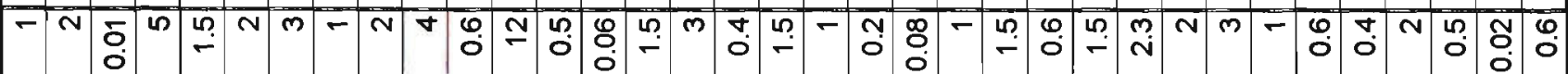

萬

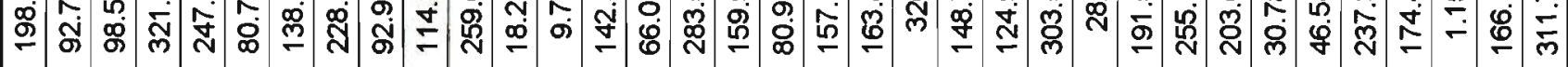
হ

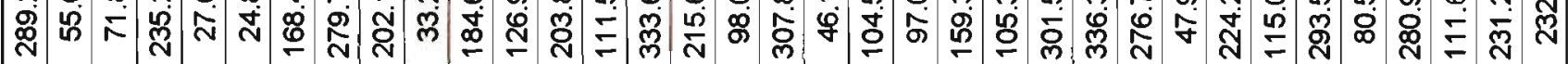

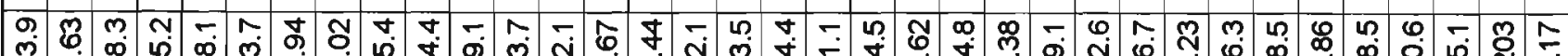

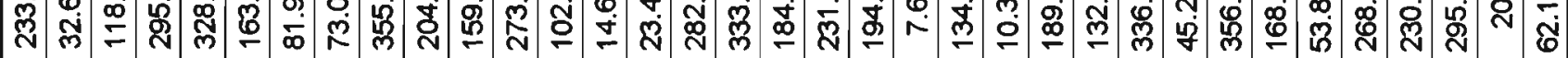

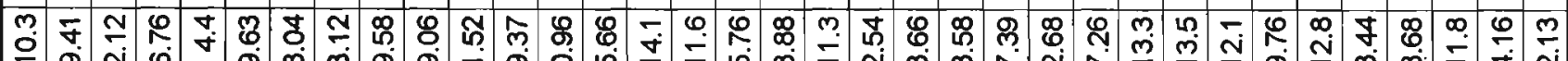

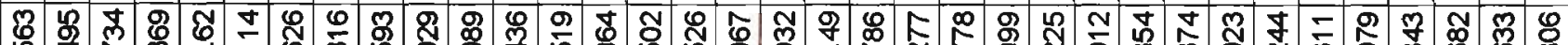
虫

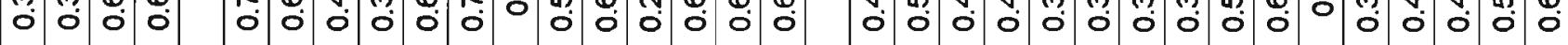

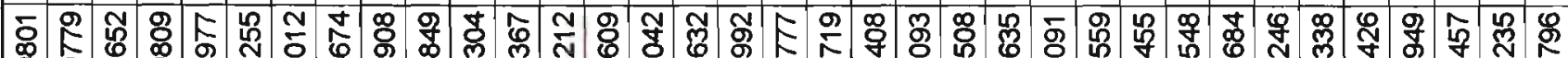
官

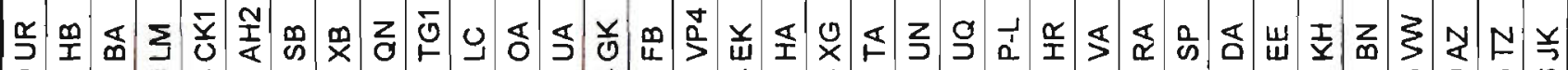

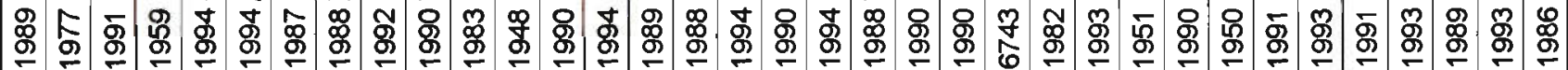

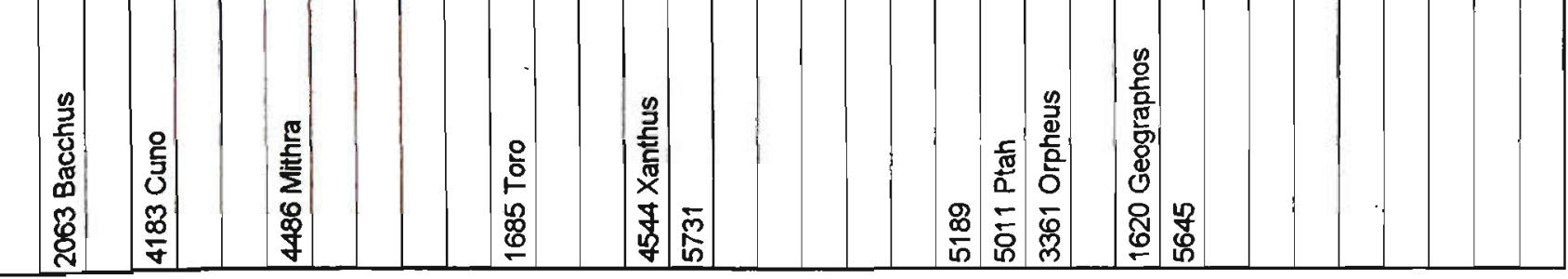




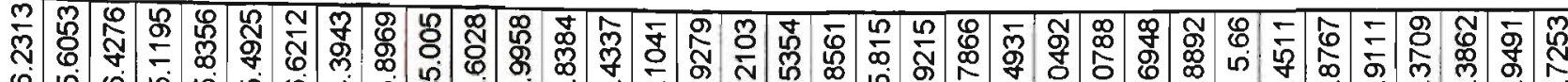

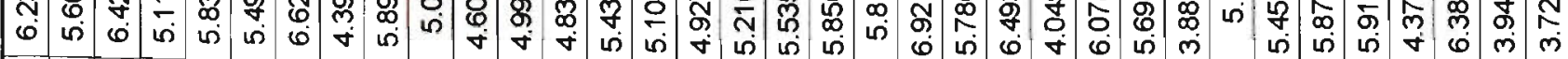

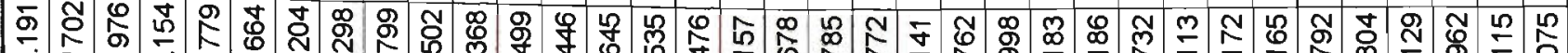

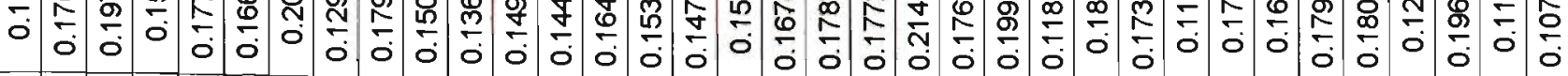

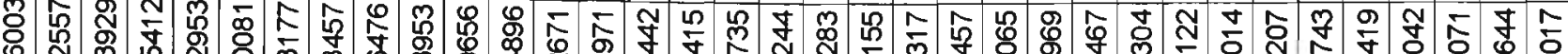

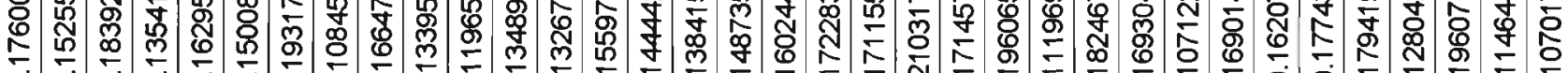

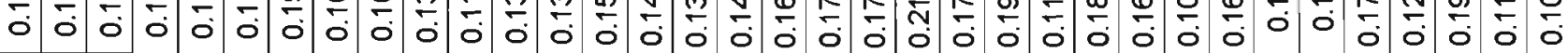

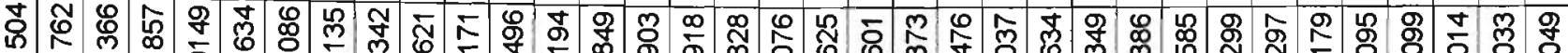
চᄒ

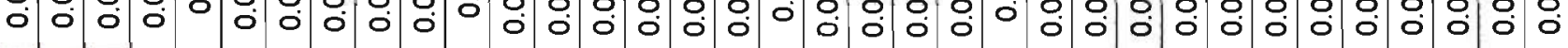

$\bar{\infty}$

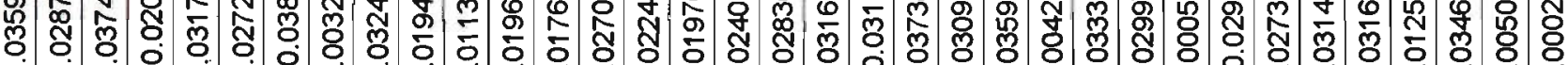

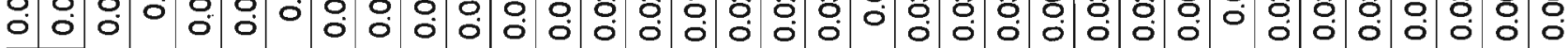

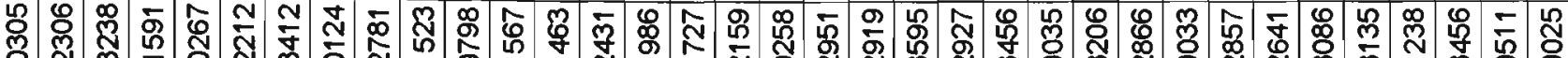

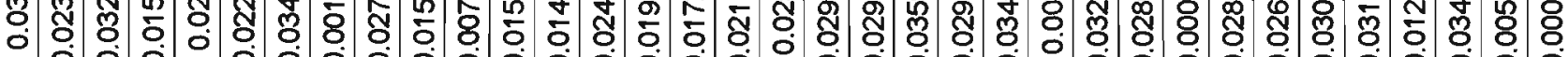

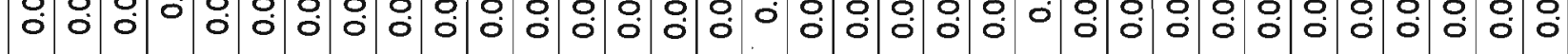

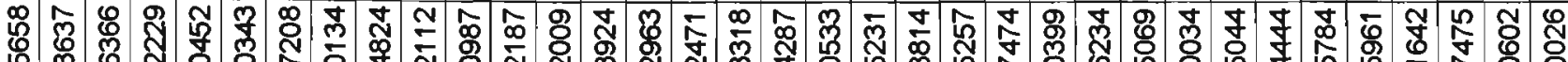

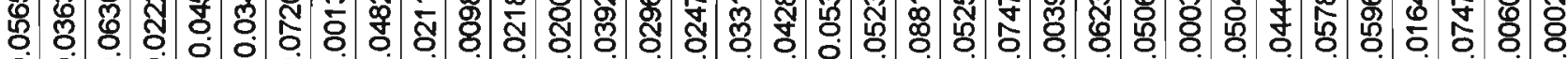

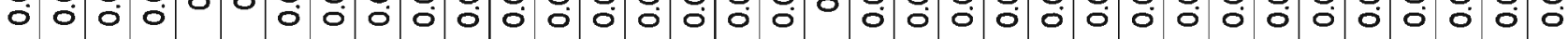

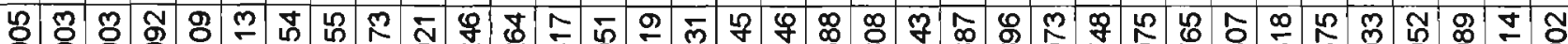
灾

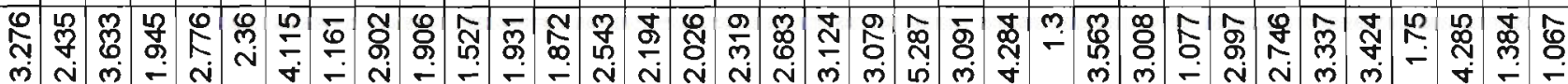

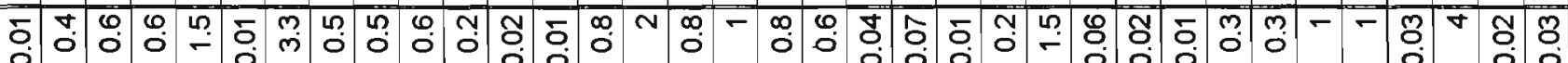

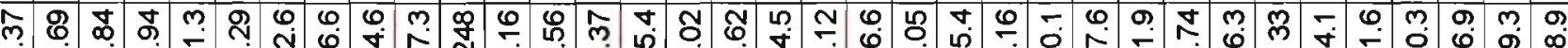

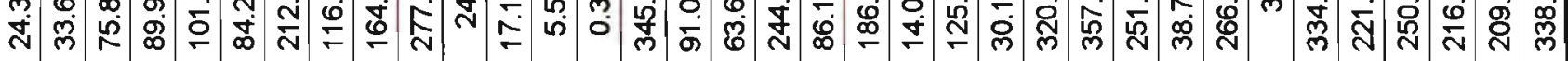
\&

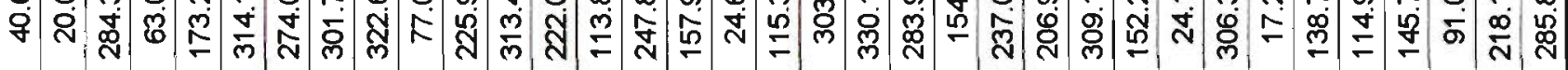

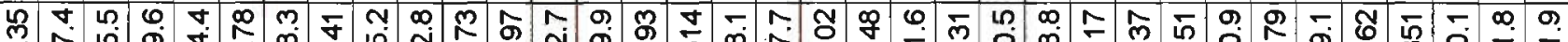

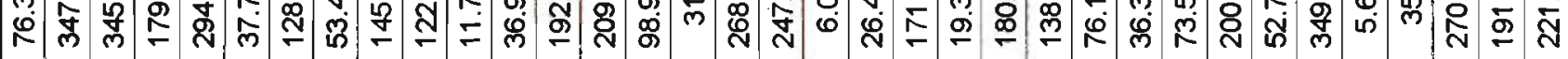

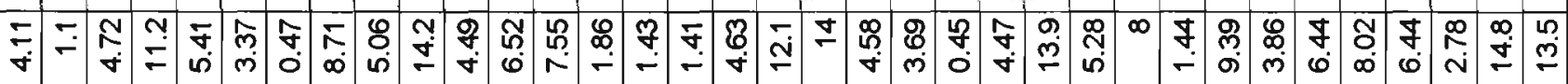
ஜ

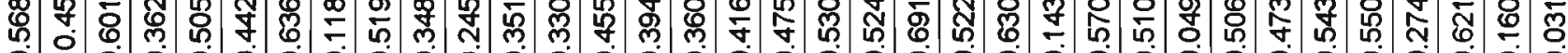

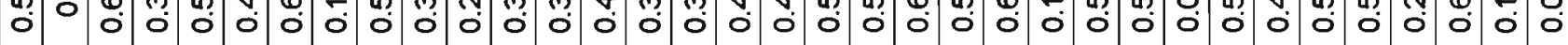

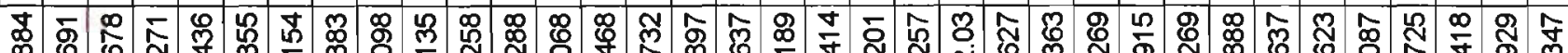

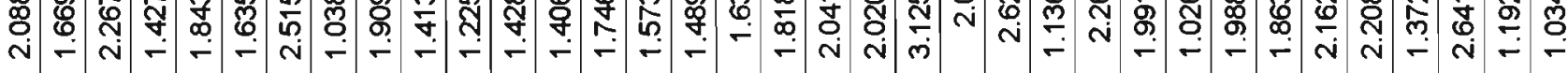

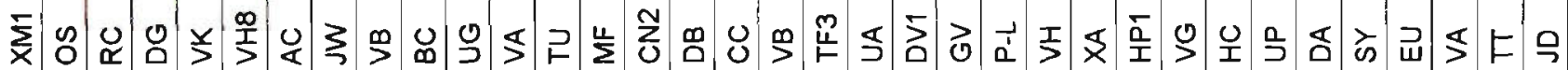

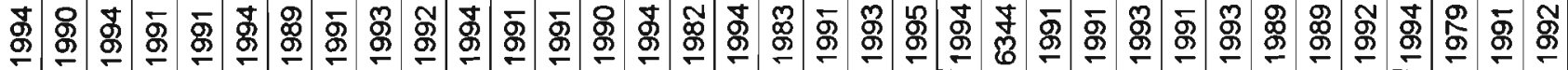

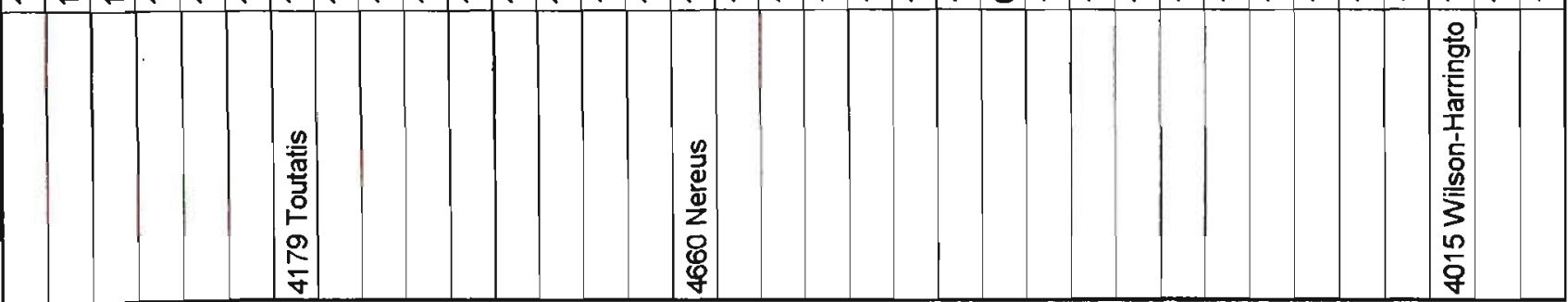




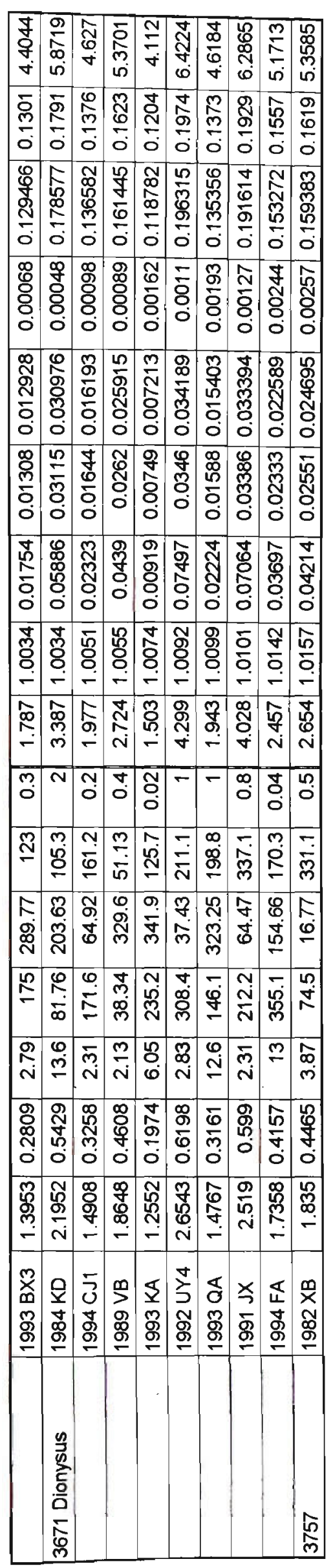

$\stackrel{\circ}{~}$ 


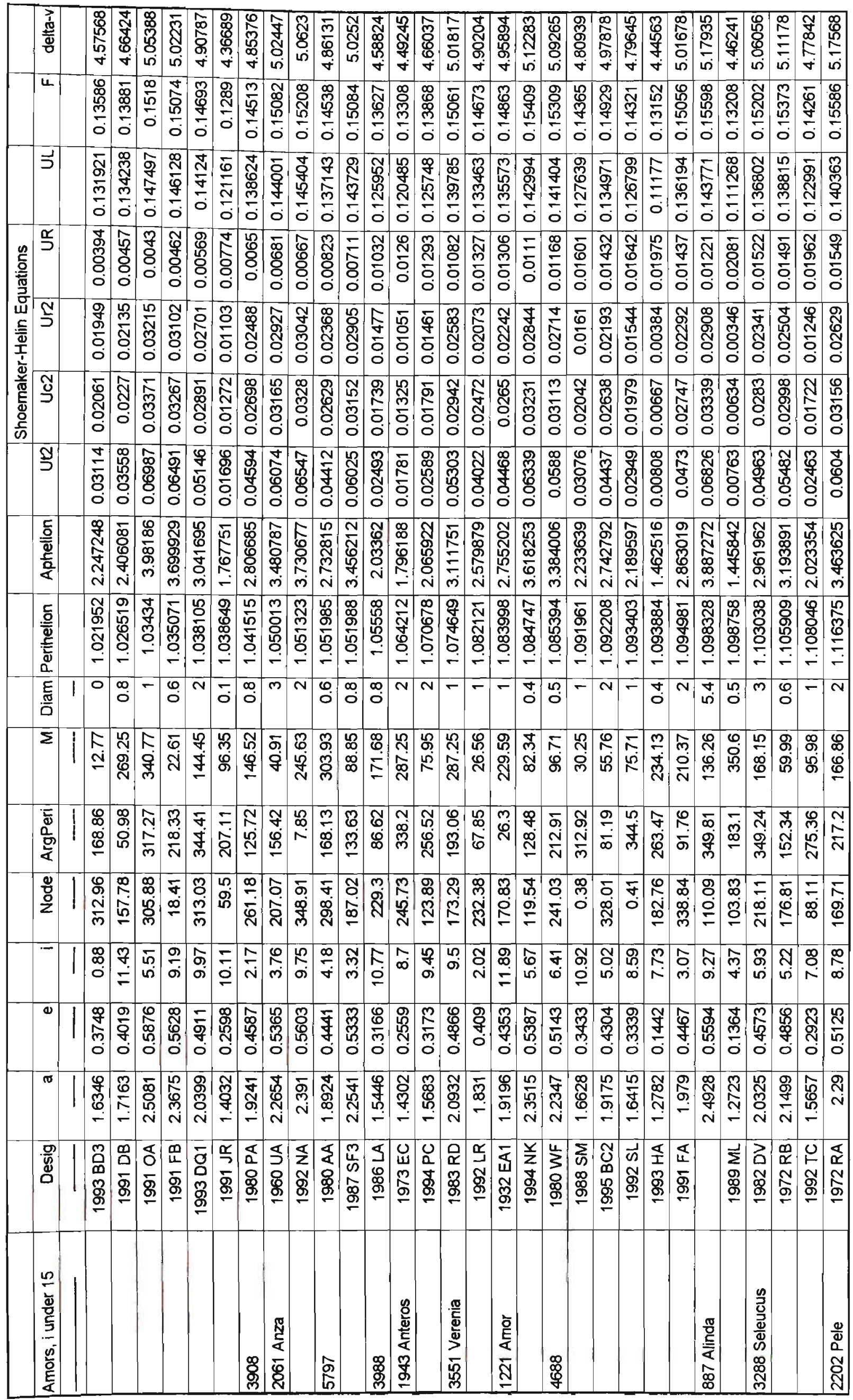




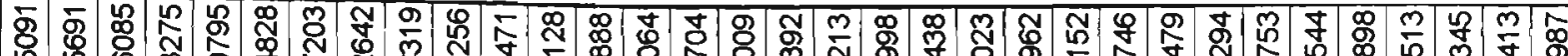

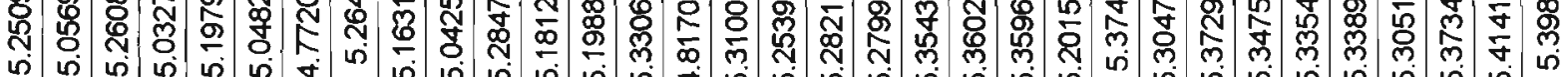

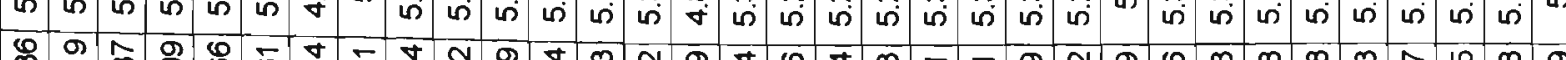

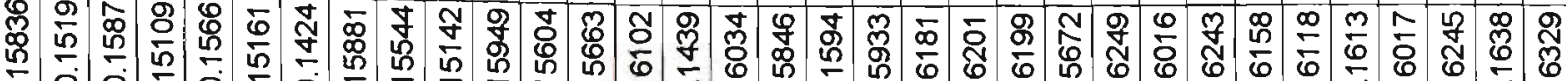

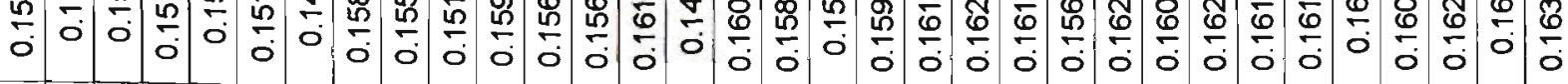

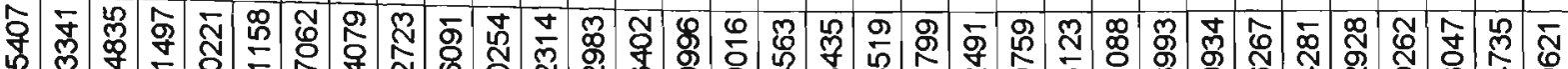

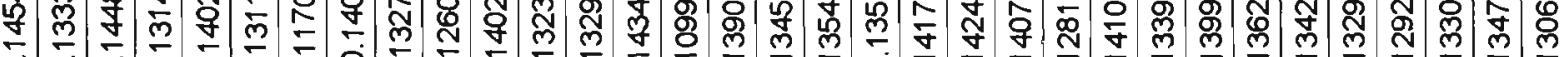

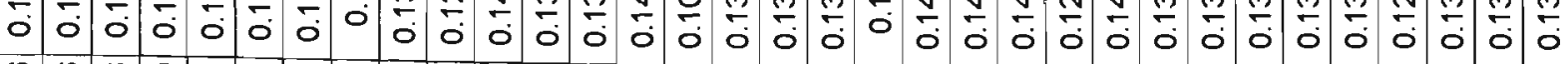
乌

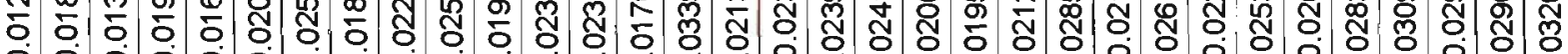

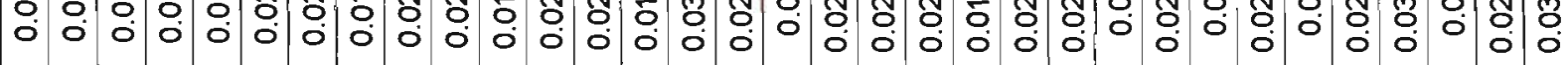
导

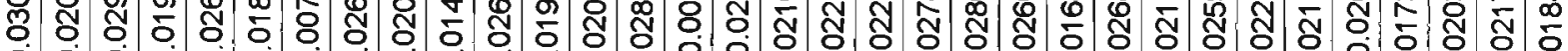
○

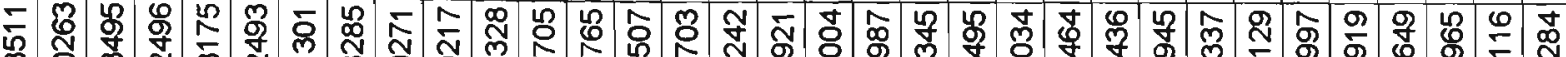

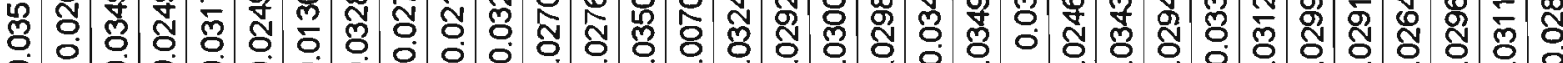

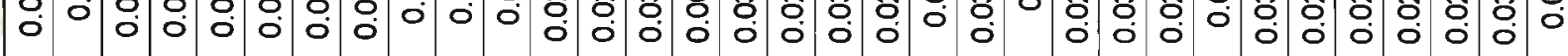

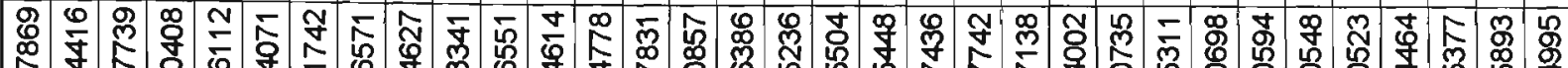

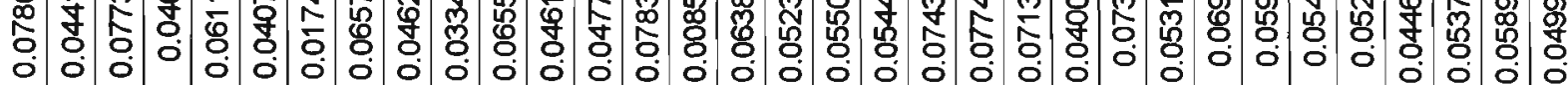
苟 员

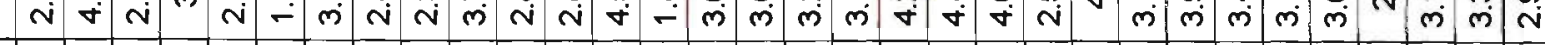

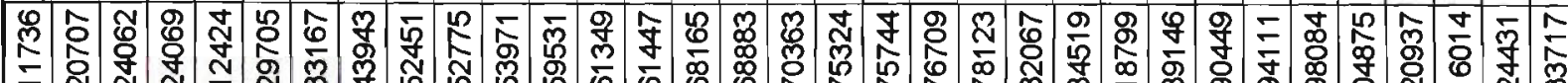

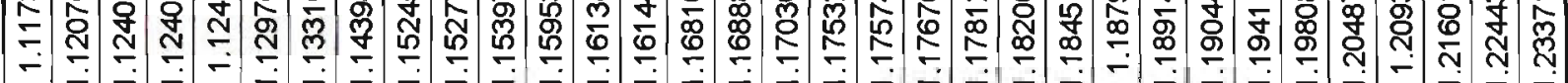

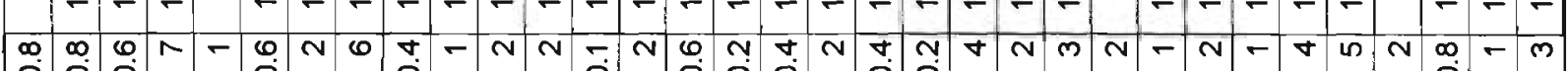

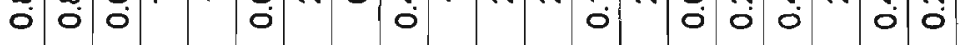

ヘு

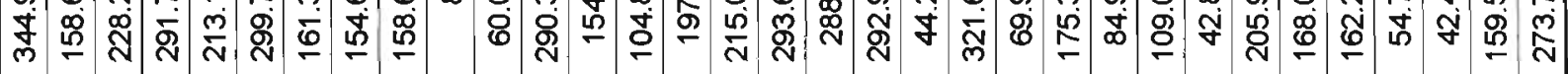

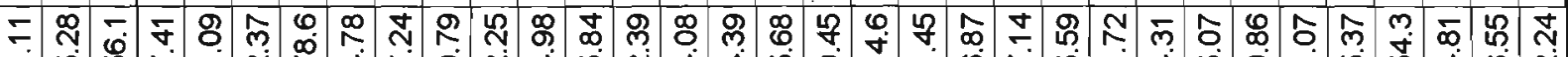

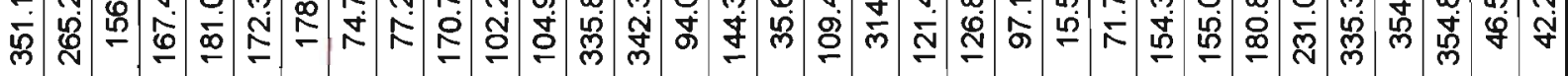

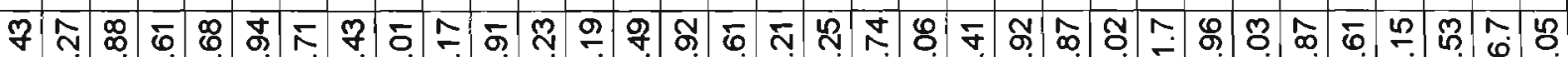

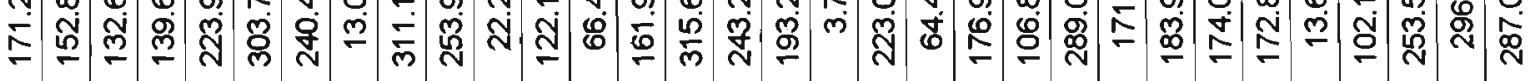

占 \%

œ

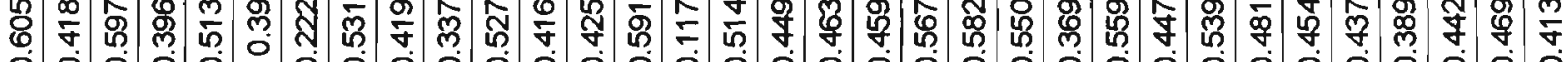

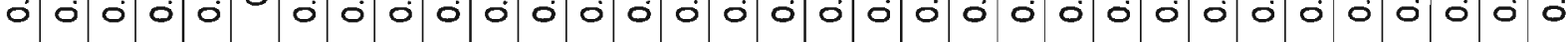

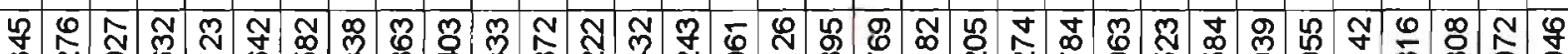
m స

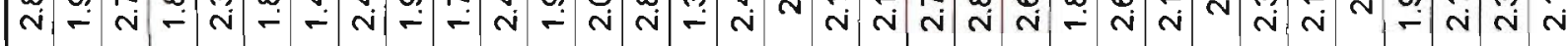

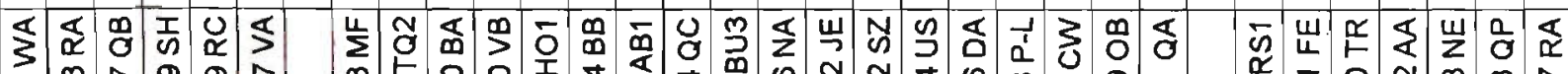

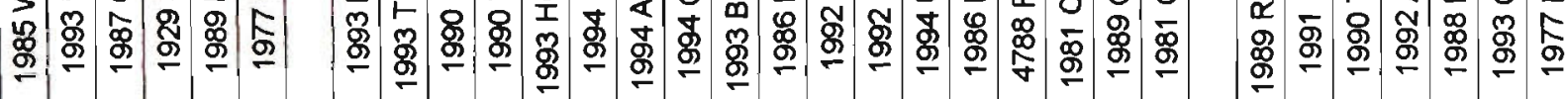

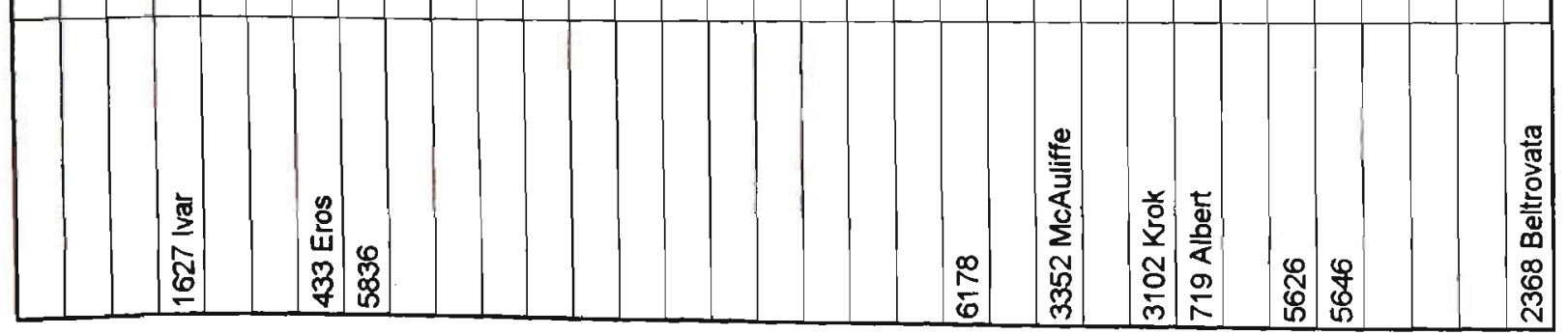




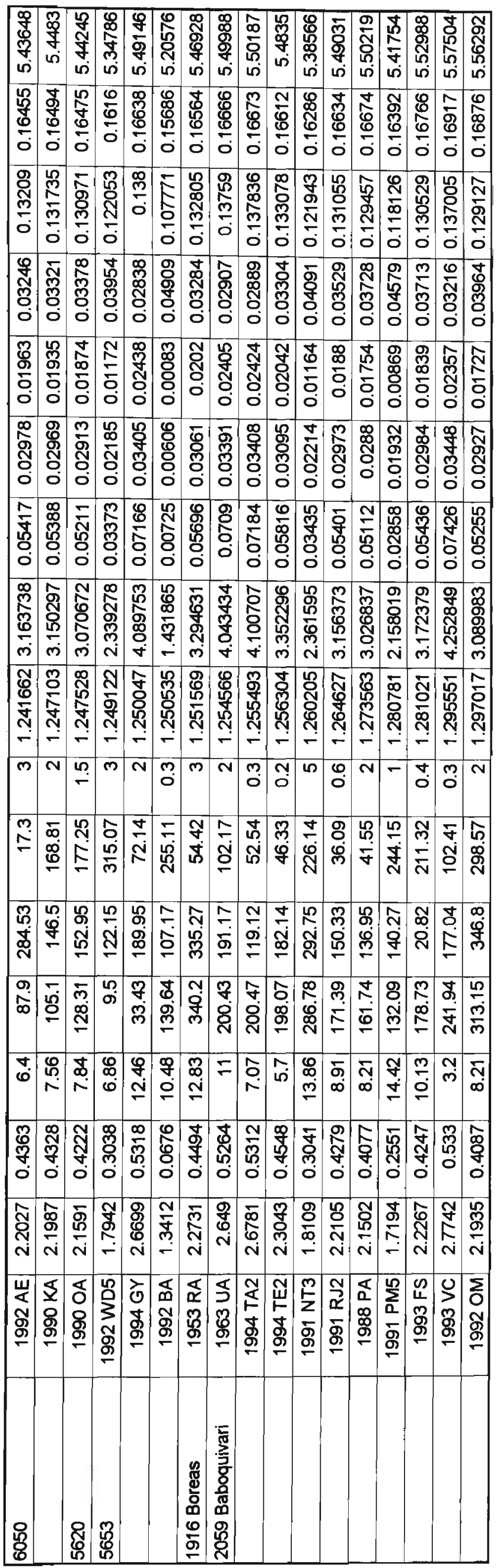

